Supporting Information

\title{
Stable axially chiral isomers of arylnaphthalene lignan glycosides with antiviral potential discovered from Justicia procumbens
}

Yang Zhao, ${ }^{\dagger, \perp}$ Chuen-Fai Ku,${ }^{\dagger}$ Xin-Ya Xu,${ }^{\dagger,} \| l$ Nga-Yi Tsang, ${ }^{\dagger}$ Yu Zhu,${ }^{\dagger}$ Chen-Liang Zhao,${ }^{\dagger}$ Kang-Lun Liu, ${ }^{\dagger}$ Chuang-Chuang Li, ${ }^{\ddagger}$ Lijun Rong, ${ }^{\S}$ Hong-Jie Zhang $*, \dagger$

${ }^{\dagger}$ School of Chinese Medicine, Hong Kong Baptist University, Kowloon Tong, Hong Kong SAR, People's Republic of China

${ }^{\ddagger}$ Shenzhen Grubbs Institute and Department of Chemistry, Southern University of Science and Technology, Shenzhen 518055, People's Republic of China

${ }^{\S}$ Department of Microbiology and Immunology, College of Medicine, University of Illinois at Chicago, 835 South Wolcott Avenue, Chicago, IL 60612, United States

${ }^{\perp}$ Current address: Department of Experimental Hematology, Beijing Institute of Radiation Medicine, Beijing 100850, People's Republic of China

${ }_{\text {Current address: Institute of Marine Drugs, Guangxi University of Chinese Medicine, }}$ No. 13 Wuhe Avenue, Nan Ning, 530200, People's Republic of China

*Corresponding author's e-mail address: zhanghj@hkbu.edu.hk 


\section{Table of Contents}

Figure S1. Scan the rotation barrier of the $6^{\prime}$-hydroxyl substituted in $6^{\prime}$-hydroxyl justicidin B and the aglycone of justatropmers.

Figure S2. The optimized structures of minimum energy conformer of $\left(1^{\prime} P\right)$-aglycone and $\left(1^{\prime} M\right)$-aglycone from the scan of the rotation barrier (no imaginary frequency was observed) .........9

Table S1. The Cartesian coordinates of optimized structure of (1'P)-aglycone. No imaginary frequency was observed.

Table S2. The Cartesian coordinates of optimized structure of (1'M)-aglycone. No imaginary frequency was observed.

Table S3. Relative free energies ${ }^{\mathrm{a}}$ and equilibrium populations ${ }^{\mathrm{b}}$ of conformers of $\left(1^{\prime} P\right)-9^{\mathrm{c}}$ 12

Figure S3. Low-energy conformers of (1'P)-9.

Figure S4. Calculated CD spectra of conformers of $\left(1^{\prime} P\right)-9$ in $\mathrm{MeOH}$ using M06-2X, CAM-B3LYP and B97X-D functionals with def2TZVP basis sets. $\sigma=0.22 \mathrm{eV}$......................13

Table S4. The Cartesian coordinates of optimized structure of (1'P)-9a.

Table S5. The Cartesian coordinates of optimized structure of $\left(1^{\prime} P\right)-9 \mathrm{~b}$. No imaginary frequency was observed.

Table S6. The Cartesian coordinates of optimized structure of (1'P)-9c. No imaginary frequency was observed. 16

Figure S5. ORTEP view of 5 showing thermal ellipsoids at the 50\% probability level. 19

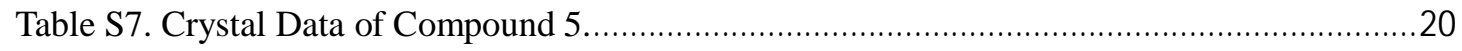

Figure S6. Plausible Biosynthetic Pathway of atropisomers 1-11_................................................21

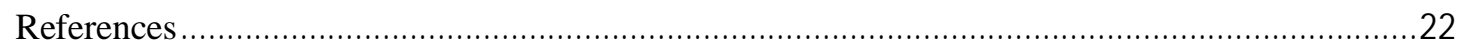

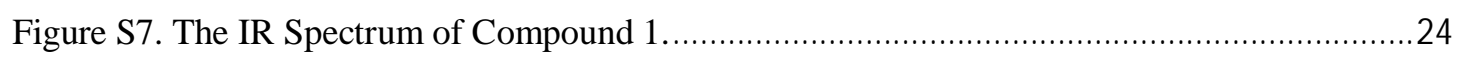

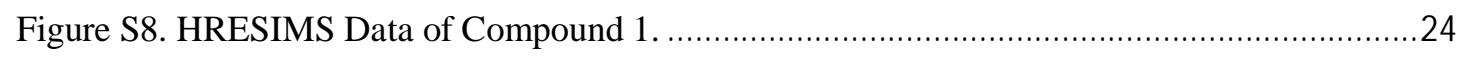

Figure S9. The ${ }^{1} \mathrm{H}$ NMR Spectrum of Compound 1 in $\mathrm{CD}_{3} \mathrm{OD}(400 \mathrm{MHz}) \ldots \ldots \ldots \ldots \ldots \ldots \ldots \ldots \ldots \ldots . . .25$

Figure S10. The ${ }^{13} \mathrm{C}\left\{{ }^{1} \mathrm{H}\right\}$ NMR Spectrum of Compound 1 in $\mathrm{CD}_{3} \mathrm{OD}(100 \mathrm{MHz}) \ldots \ldots \ldots \ldots \ldots \ldots \ldots . . . . .25$

Figure S11. The HSQC Spectrum of Compound 1 in $\mathrm{CD}_{3} \mathrm{OD}\left(400 \mathrm{MHz}\right.$ for $\left.{ }^{1} \mathrm{H}\right)$.........................26

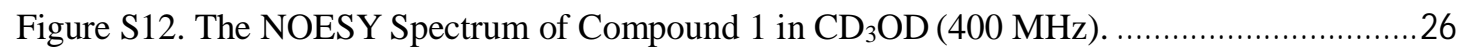

Figure S13. The HMBC Spectrum of Compound 1 in $\mathrm{CD}_{3} \mathrm{OD}\left(400 \mathrm{MHz}\right.$ for $\left.{ }^{1} \mathrm{H}\right)$.......................27

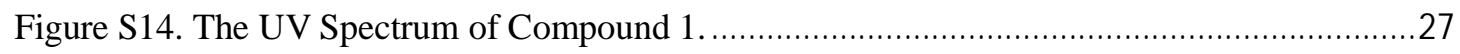

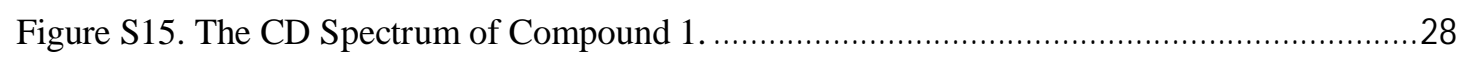

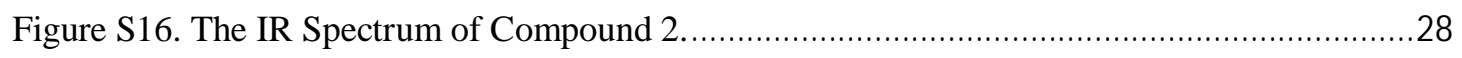

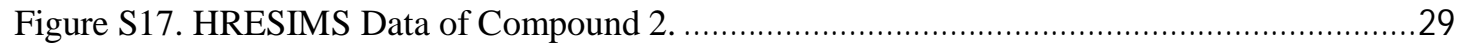

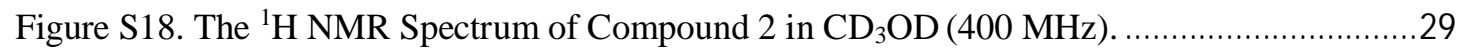

Figure S19. The ${ }^{13} \mathrm{C}\left\{{ }^{1} \mathrm{H}\right\}$ NMR Spectrum of Compound 2 in $\mathrm{CD}_{3} \mathrm{OD}(100 \mathrm{MHz})$........................30 
Figure S20. The HMBC Spectrum of Compound 2 in $\mathrm{CD}_{3} \mathrm{OD}\left(400 \mathrm{MHz}\right.$ for $\left.{ }^{1} \mathrm{H}\right)$. ..30

Figure S21. The UV Spectrum of Compound 2 ..............................................................31

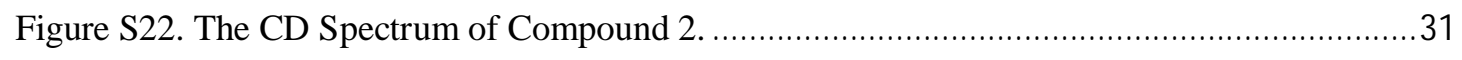

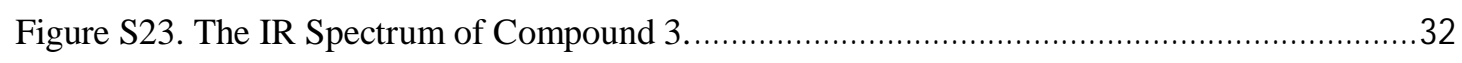

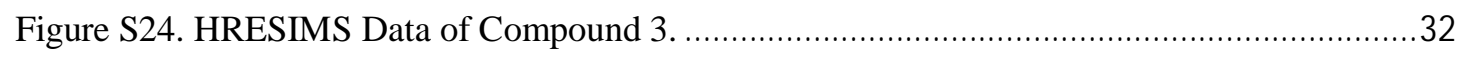

Figure S25. The ${ }^{1} \mathrm{H}$ NMR Spectrum of Compound 3 in $\mathrm{CD}_{3} \mathrm{OD}(400 \mathrm{MHz})$.............................33

Figure S26. The ${ }^{13} \mathrm{C}\left\{{ }^{1} \mathrm{H}\right\}$ NMR Spectrum of Compound 3 in $\mathrm{CD}_{3} \mathrm{OD}(100 \mathrm{MHz}) \ldots \ldots \ldots \ldots \ldots \ldots . . . . . . . . . . . . . .33$

Figure S27. The HSQC Spectrum of Compound 3 in $\mathrm{CD}_{3} \mathrm{OD}\left(400 \mathrm{MHz}\right.$ for $\left.{ }^{1} \mathrm{H}\right) \ldots \ldots \ldots \ldots \ldots \ldots \ldots . . . . . . . . . . . . . .34$

Figure S28. The ${ }^{1} \mathrm{H}-{ }^{1} \mathrm{H}$ COSY Spectrum of Compound 3 in $\mathrm{CD}_{3} \mathrm{OD}(400 \mathrm{MHz})$.........................34

Figure S29. The HMBC Spectrum of Compound 3 in $\mathrm{CD}_{3} \mathrm{OD}\left(400 \mathrm{MHz}\right.$ for $\left.{ }^{1} \mathrm{H}\right) \ldots \ldots \ldots \ldots \ldots \ldots . . . . . . . . . . . . . .35$

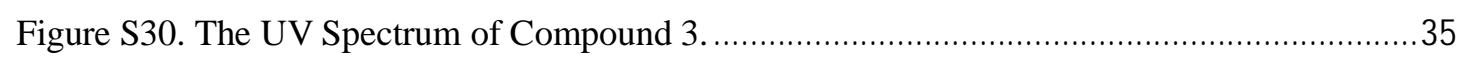

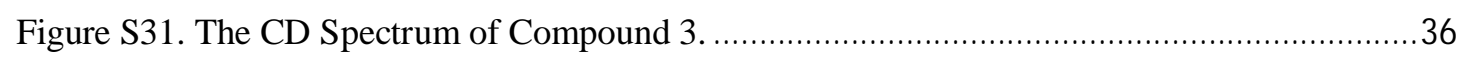

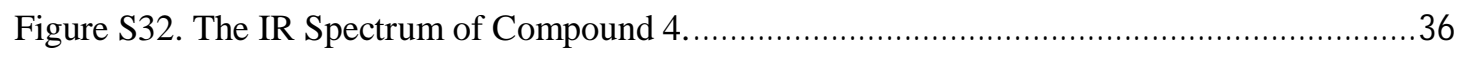

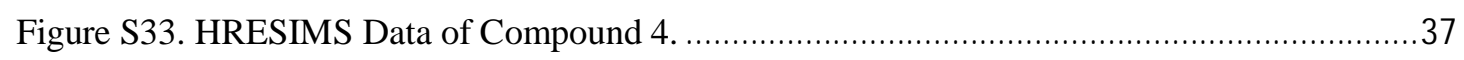

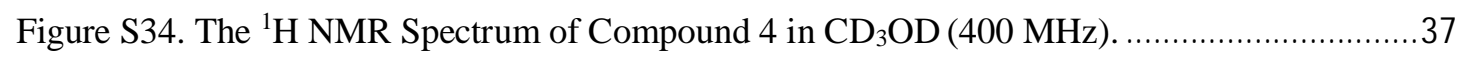

Figure S35. The ${ }^{13} \mathrm{C}\left\{{ }^{1} \mathrm{H}\right\}$ NMR Spectrum of Compound 4 in $\mathrm{CD}_{3} \mathrm{OD}(100 \mathrm{MHz})$......................38

Figure S36. The HSQC Spectrum of Compound 4 in $\mathrm{CD}_{3} \mathrm{OD}\left(400 \mathrm{MHz}\right.$ for $\left.{ }^{1} \mathrm{H}\right)$.........................38

Figure S37. The ${ }^{1} \mathrm{H}-{ }^{1} \mathrm{H}$ COSY Spectrum of Compound 4 in $\mathrm{CD}_{3} \mathrm{OD}(400 \mathrm{MHz})$.........................39

Figure S38. The HMBC Spectrum of Compound 4 in $\mathrm{CD}_{3} \mathrm{OD}\left(400 \mathrm{MHz}\right.$ for $\left.{ }^{1} \mathrm{H}\right) \ldots \ldots \ldots \ldots \ldots \ldots . . . . . . . . . . . . . .39$

Figure S39. The UV Spectrum of Compound 4 .....................................................................40

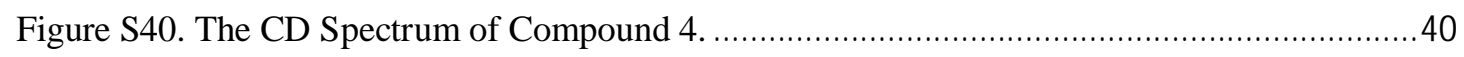

Figure S41. The IR Spectrum of Compound 5 ............................................................... 41

Figure S42. HRESIMS Data of Compound 5 . ..............................................................41

Figure S43. The ${ }^{1} \mathrm{H}$ NMR Spectrum of Compound 5 in $\mathrm{CD}_{3} \mathrm{OD}(400 \mathrm{MHz})$..............................42

Figure S44. The ${ }^{13} \mathrm{C}\left\{{ }^{1} \mathrm{H}\right\}$ NMR Spectrum of Compound 5 in $\mathrm{CD}_{3} \mathrm{OD}(100 \mathrm{MHz})$......................42

Figure S45. The HSQC Spectrum of Compound 5 in $\mathrm{CD}_{3} \mathrm{OD}\left(400 \mathrm{MHz}\right.$ for $\left.{ }^{1} \mathrm{H}\right) \ldots \ldots \ldots \ldots \ldots \ldots \ldots . . . . . . . . . . . . . . .33$

Figure S46. The ${ }^{1} \mathrm{H}-{ }^{1} \mathrm{H}$ COSY Spectrum of Compound 5 in $\mathrm{CD}_{3} \mathrm{OD}(400 \mathrm{MHz}) . \ldots \ldots \ldots \ldots \ldots \ldots . . . . . . . . . . . . . . .33$

Figure S47. The HMBC Spectrum of Compound 5 in $\mathrm{CD}_{3} \mathrm{OD}\left(400 \mathrm{MHz}\right.$ for $\left.{ }^{1} \mathrm{H}\right)$.....................44

Figure S48. The UV Spectrum of Compound 5 ...............................................................44

Figure S49. The CD Spectrum of Compound 5 . ................................................................. 45

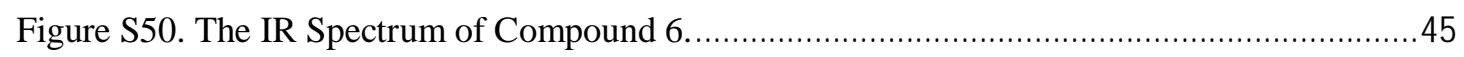

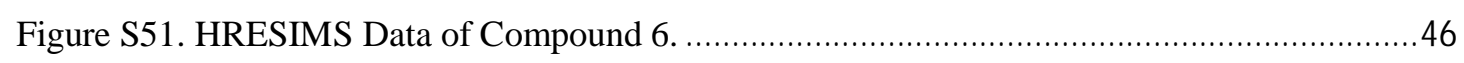

Figure S52. The ${ }^{1} \mathrm{H}$ NMR Spectrum of Compound 6 in $\mathrm{CD}_{3} \mathrm{OD}(400 \mathrm{MHz})$...............................46

Figure S53. The ${ }^{13} \mathrm{C}\left\{{ }^{1} \mathrm{H}\right\}$ NMR Spectrum of Compound 6 in $\mathrm{CD}_{3} \mathrm{OD}(100 \mathrm{MHz}) \ldots \ldots \ldots \ldots \ldots \ldots . . . . . . . . . . . . . .47$ 
Figure S54. The HSQC Spectrum of Compound 6 in $\mathrm{CD}_{3} \mathrm{OD}\left(400 \mathrm{MHz}\right.$ for $\left.{ }^{1} \mathrm{H}\right)$. . .47

Figure S55. The ${ }^{1} \mathrm{H}-{ }^{1} \mathrm{H}$ COSY Spectrum of Compound 6 in $\mathrm{CD}_{3} \mathrm{OD}(400 \mathrm{MHz})$......................48

Figure S56. The HMBC Spectrum of Compound 6 in $\mathrm{CD}_{3} \mathrm{OD}\left(400 \mathrm{MHz}\right.$ for $\left.{ }^{1} \mathrm{H}\right) \ldots \ldots \ldots \ldots \ldots \ldots \ldots . . . . . . . . . . . . .48$

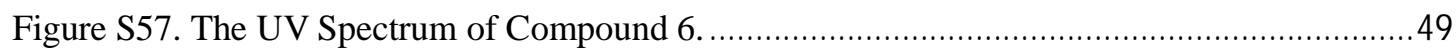

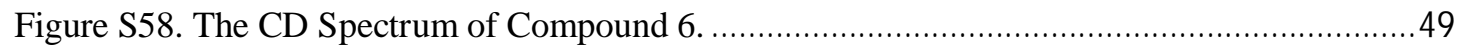

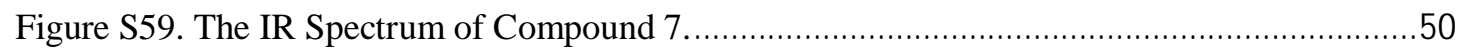

Figure S60. HRESIMS Data of Compound 7. .....................................................................50

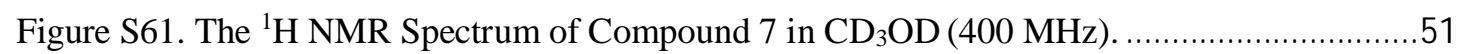

Figure S62. The ${ }^{13} \mathrm{C}\left\{{ }^{1} \mathrm{H}\right\}$ NMR Spectrum of Compound 7 in $\mathrm{CD}_{3} \mathrm{OD}(100 \mathrm{MHz}) \ldots . . . . . . . . . . . . . . . . . .51$

Figure S63. The HSQC Spectrum of Compound 7 in $\mathrm{CD}_{3} \mathrm{OD}\left(400 \mathrm{MHz}\right.$ for $\left.{ }^{1} \mathrm{H}\right)$.............................

Figure S64. The ${ }^{1} \mathrm{H}-{ }^{1} \mathrm{H}$ COSY Spectrum of Compound 7 in $\mathrm{CD}_{3} \mathrm{OD}(400 \mathrm{MHz}) . \ldots \ldots \ldots \ldots \ldots \ldots . . . . . . . . . . . . . .52$

Figure S65. The HMBC Spectrum of Compound 7 in $\mathrm{CD}_{3} \mathrm{OD}\left(400 \mathrm{MHz}\right.$ for $\left.{ }^{1} \mathrm{H}\right)$........................53

Figure S66. The UV Spectrum of Compound 7. ...........................................................53

Figure S67. The CD Spectrum of Compound 7. .................................................................54

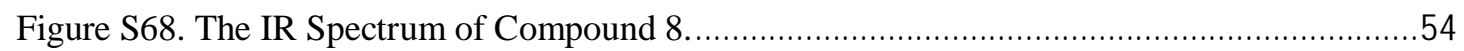

Figure S69. HRESIMS Data of Compound 8. ......................................................................55

Figure S70. The ${ }^{1} \mathrm{H}$ NMR Spectrum of Compound 8 in $\mathrm{CD}_{3} \mathrm{OD}(400 \mathrm{MHz})$...............................55

Figure S71. The ${ }^{13} \mathrm{C}\left\{{ }^{1} \mathrm{H}\right\}$ NMR Spectrum of Compound 8 in $\mathrm{CD}_{3} \mathrm{OD}(100 \mathrm{MHz}) \ldots \ldots \ldots \ldots \ldots \ldots . . . . . . . . . . . . .56$

Figure S72. The HSQC Spectrum of Compound 8 in $\mathrm{CD}_{3} \mathrm{OD}\left(400 \mathrm{MHz}\right.$ for $\left.{ }^{1} \mathrm{H}\right)$.........................56

Figure S73. The ${ }^{1} \mathrm{H}-{ }^{1} \mathrm{H}$ COSY Spectrum of Compound 8 in $\mathrm{CD}_{3} \mathrm{OD}(400 \mathrm{MHz})$.......................57

Figure S74. The HMBC Spectrum of Compound 8 in $\mathrm{CD}_{3} \mathrm{OD}\left(400 \mathrm{MHz}\right.$ for $\left.{ }^{1} \mathrm{H}\right)$......................57

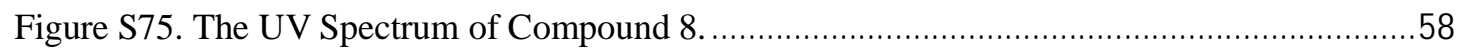

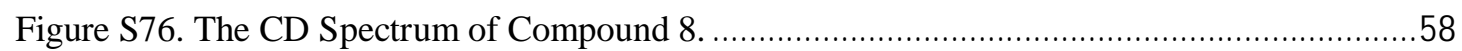

Figure S77. The IR Spectrum of Compound 9 ..................................................................59

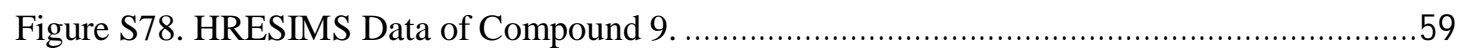

Figure S79. The ${ }^{1} \mathrm{H}$ NMR Spectrum of Compound 9 in $\mathrm{CD}_{3} \mathrm{OD}(400 \mathrm{MHz})$..............................60

Figure S80. The ${ }^{13} \mathrm{C}\left\{{ }^{1} \mathrm{H}\right\}$ NMR Spectrum of Compound 9 in $\mathrm{CD}_{3} \mathrm{OD}(100 \mathrm{MHz}) \ldots \ldots \ldots \ldots \ldots \ldots . . . . . . . . . . . . . . .60$

Figure S81. The NOESY Spectrum of Compound 9 in $\mathrm{CD}_{3} \mathrm{OD}(400 \mathrm{MHz})$............................61

Figure S82. The UV Spectrum of Compound 9. ................................................................61

Figure S83. The CD Spectrum of Compound 9. ..............................................................62

Figure S84. The IR Spectrum of Compound 10................................................................62

Figure S85. HRESIMS Data of Compound 10 ................................................................63

Figure S86. The ${ }^{1} \mathrm{H}$ NMR Spectrum of Compound 10 in $\mathrm{CD}_{3} \mathrm{OD}(400 \mathrm{MHz})$............................63

Figure S87. The ${ }^{13} \mathrm{C}\left\{{ }^{1} \mathrm{H}\right\}$ NMR Spectrum of Compound 10 in $\mathrm{CD}_{3} \mathrm{OD}(100 \mathrm{MHz}) \ldots \ldots \ldots \ldots \ldots \ldots . . . . . . . . . . . .64$ 
Figure S88. The NOESY Spectrum of Compound 10 in $\mathrm{CD}_{3} \mathrm{OD}(400 \mathrm{MHz})$.

Figure S89. The UV Spectrum of Compound 10.....................................................................65

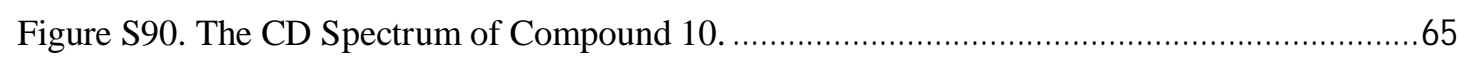

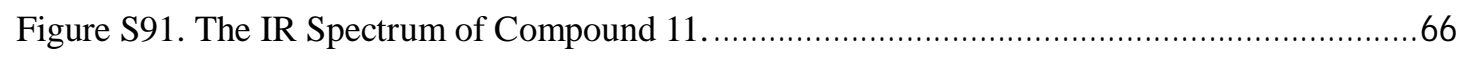

Figure S92. HRESIMS Data of Compound 11....................................................................66

Figure S93. The ${ }^{1} \mathrm{H}$ NMR Spectrum of Compound 11 in $\mathrm{CD}_{3} \mathrm{OD}(400 \mathrm{MHz})$...........................67

Figure S94. The ${ }^{13} \mathrm{C}\left\{{ }^{1} \mathrm{H}\right\}$ NMR Spectrum of Compound 11 in $\mathrm{CD}_{3} \mathrm{OD}(100 \mathrm{MHz})$.....................67

Figure S95. The HMBC Spectrum of Compound 11 in $\mathrm{CD}_{3} \mathrm{OD}\left(400 \mathrm{MHz}\right.$ for $\left.{ }^{1} \mathrm{H}\right)$. ......................68

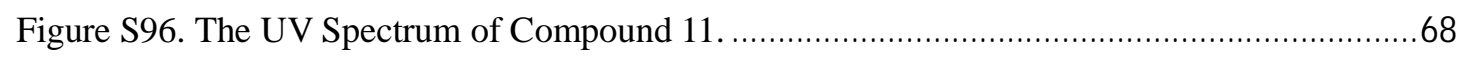

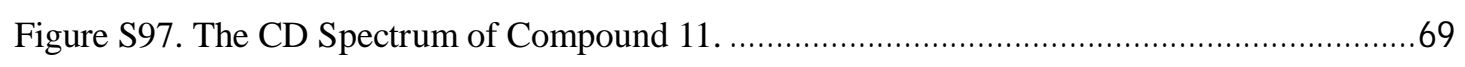

Figure S98. The ${ }^{1} \mathrm{H}$ NMR Spectrum of Compound 12 in $\mathrm{CD}_{3} \mathrm{OD}(400 \mathrm{MHz})$..............................69

Figure S99. HRESIMS Data of intermediate Compound 14................................................... 70

Figure S100. HRESIMS Data of intermediate Compound 15 ......................................................70

Figure S101. HRESIMS Data of intermediate Compound 17 .....................................................70

Figure S102. HRESIMS Data of intermediate Compound 18 ..................................................... 71

Figure S103. HRESIMS Data of intermediate Compound 19.....................................................

Figure S104. The ${ }^{1} \mathrm{H}$ NMR Spectrum of intermediate Compound 19 in DMSO-d $d_{6}(400 \mathrm{MHz}) \ldots . . .72$

Figure S105. HRESIMS Data of intermediate Compound 20................................................... 72

Figure S106. The ${ }^{1} \mathrm{H}$ NMR Spectrum of intermediate Compound 20 in DMSO-d $d_{6}(400 \mathrm{MHz}) \ldots . . .73$

Figure S107. HRESIMS Data of intermediate Compound 21 ......................................................73

Figure S108. The ${ }^{1} \mathrm{H}$ NMR Spectrum of intermediate Compound 21 in $\mathrm{CDCl}_{3}(400 \mathrm{MHz})$..........74

Figure S109. The ${ }^{13} \mathrm{C}\left\{{ }^{1} \mathrm{H}\right\}$ NMR Spectrum of intermediate Compound 21 in $\mathrm{CDCl}_{3}(100 \mathrm{MHz}) .74$

Figure S110. HRESIMS Data of intermediate Compound 22a....................................................75

Figure S111. The ${ }^{1} \mathrm{H}$ NMR Spectrum of intermediate Compound 22a in $\mathrm{CDCl}_{3}(400 \mathrm{MHz})$.........75

Figure S112. The ${ }^{13} \mathrm{C}\left\{{ }^{1} \mathrm{H}\right\}$ NMR Spectrum of intermediate Compound 22a in $\mathrm{CDCl}_{3}(100 \mathrm{MHz})$.

Figure S113. HRESIMS Data of intermediate Compound 22b. ................................................76

Figure S114. The ${ }^{1} \mathrm{H}$ NMR Spectrum of intermediate Compound 22b in $\mathrm{CDCl}_{3}(400 \mathrm{MHz}) \ldots \ldots . . .77$

Figure S115. The ${ }^{13} \mathrm{C}\left\{{ }^{1} \mathrm{H}\right\}$ NMR Spectrum of intermediate Compound $22 \mathrm{~b}$ in $\mathrm{CDCl}_{3}(100 \mathrm{MHz})$.

Figure S116. HRESIMS Data of synthetic 1 ................................................................... 78

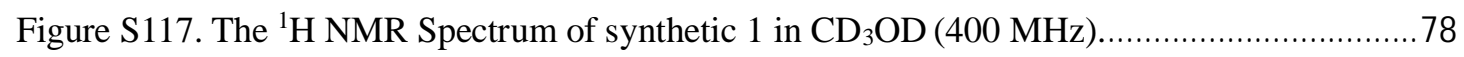

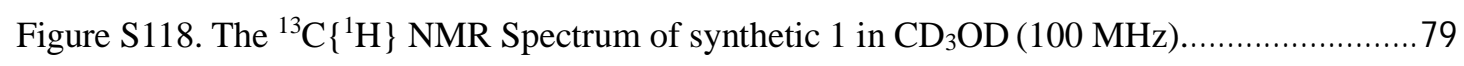

Figure S119. The CD Spectrum of synthetic 1 in $\mathrm{MeOH}$.......................................................79 


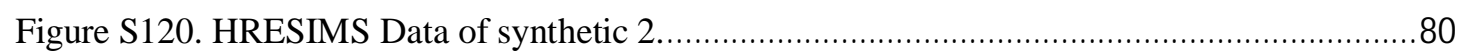

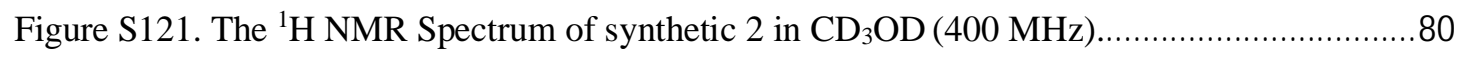

Figure S122. The ${ }^{13} \mathrm{C}$ NMR Spectrum of synthetic 2 in $\mathrm{CD}_{3} \mathrm{OD}(100 \mathrm{MHz})$................................81

Figure S123. The CD Spectrum of synthetic 2 in $\mathrm{MeOH}$......................................................... 81

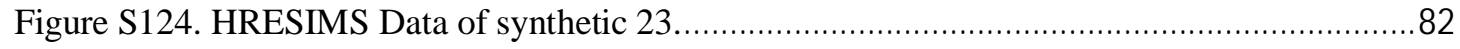

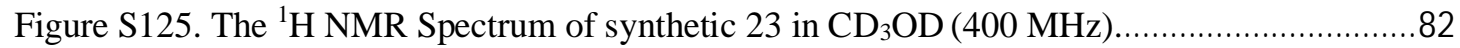

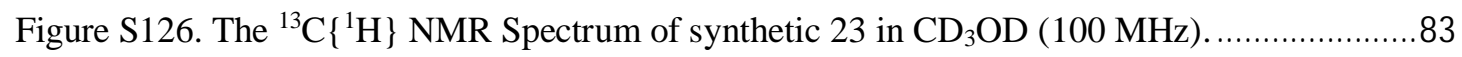

Figure S127. The CD Spectrum of synthetic 23 in $\mathrm{MeOH}$...................................................83

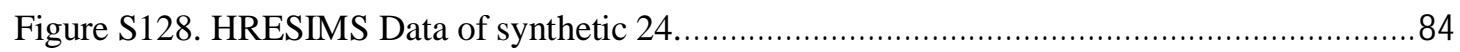

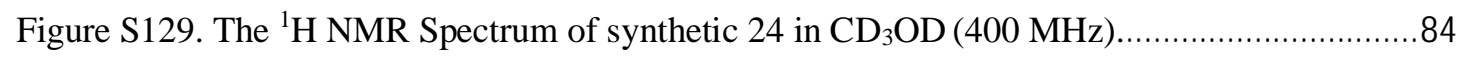

Figure S130. The ${ }^{13} \mathrm{C}\left\{{ }^{1} \mathrm{H}\right\}$ NMR Spectrum of synthetic 24 in $\mathrm{CD}_{3} \mathrm{OD}(100 \mathrm{MHz})$.....................85

Figure S131. The CD Spectrum of synthetic 24 in $\mathrm{MeOH}$..................................................... 85 
(A)

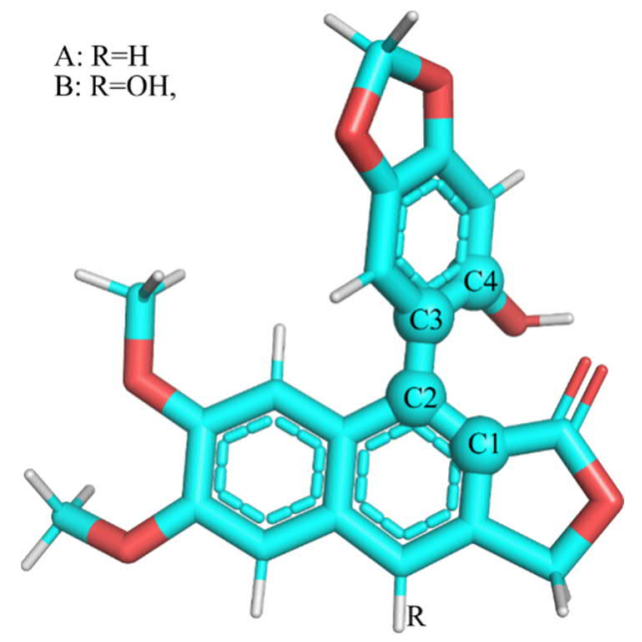

(B)

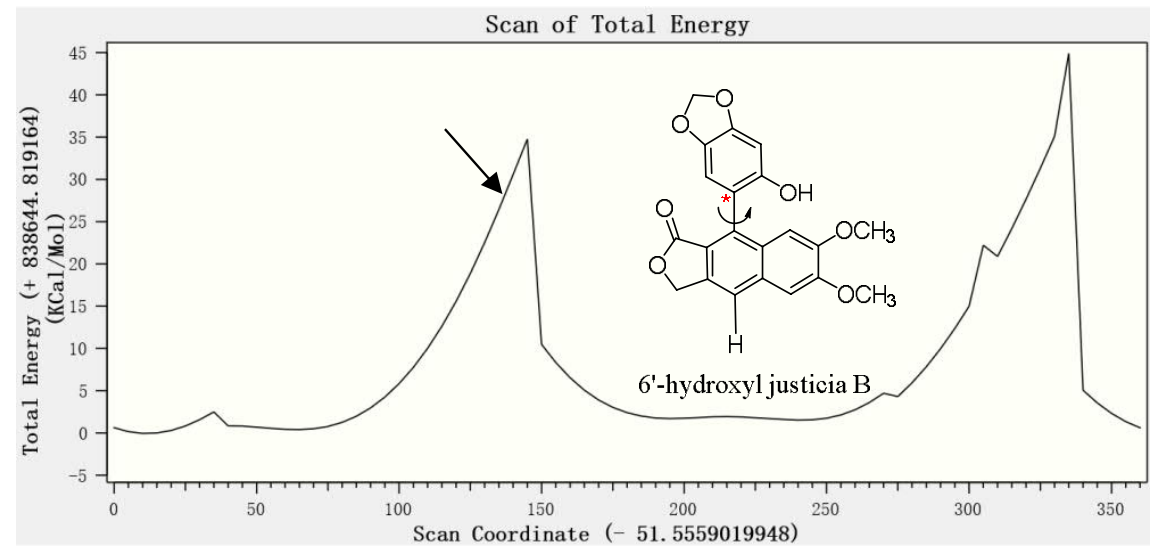

(C)

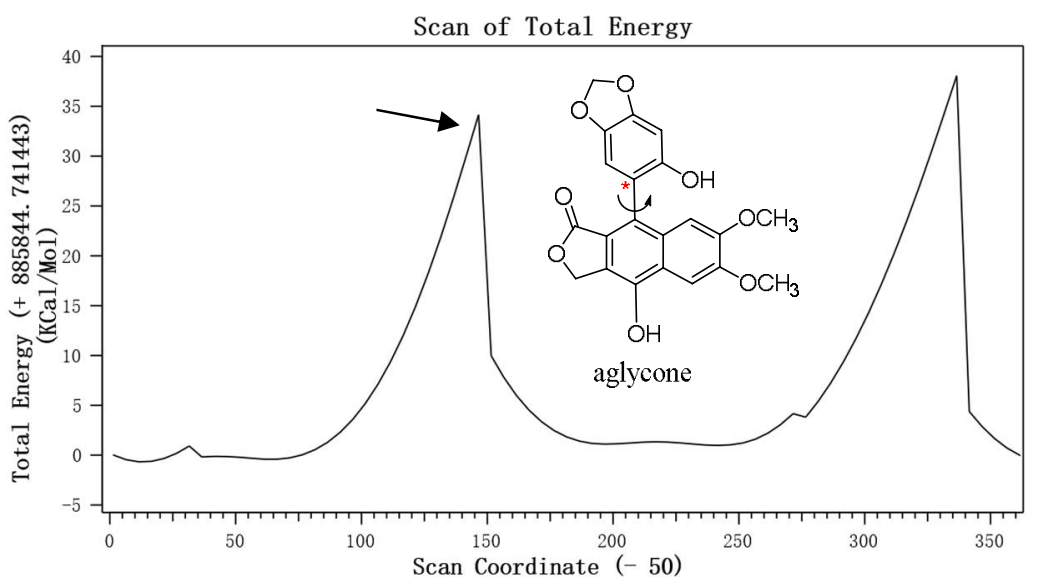


(D)
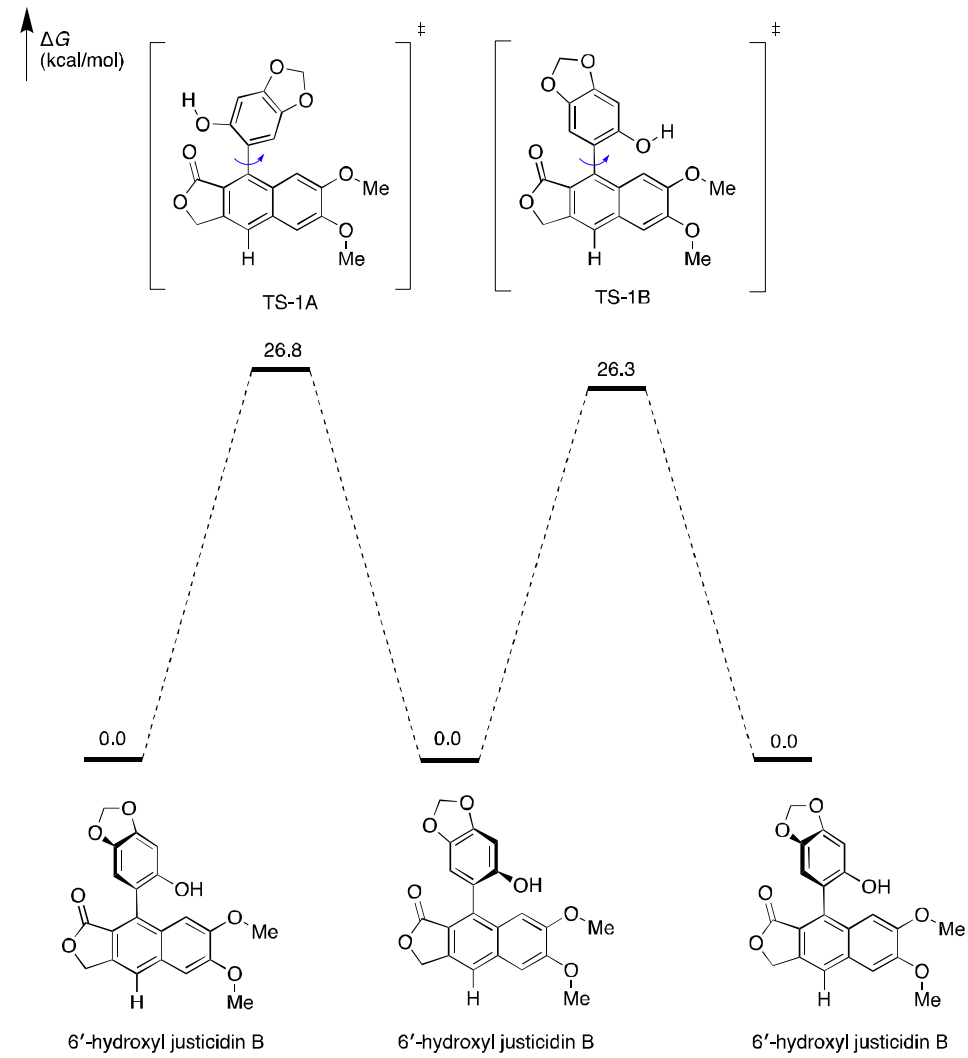

B3LYP-D3(BJ)/6-311+G(d,p)-SMD(methanol)/B3LYP/6-31G(d)

(E)
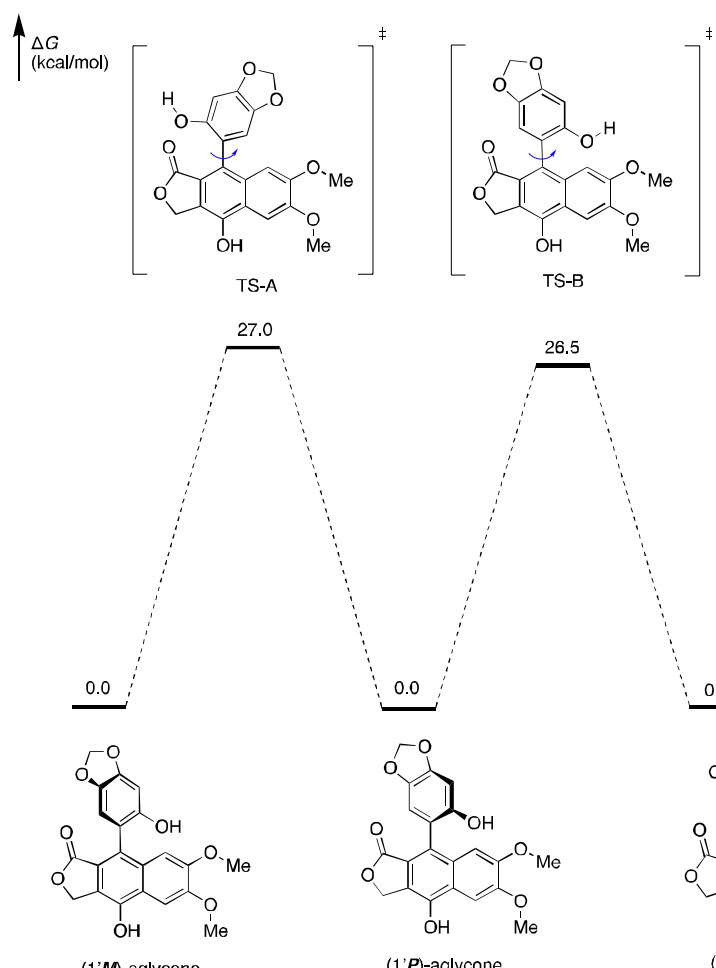

26.5

-aglycone

(1'P)-aglycone

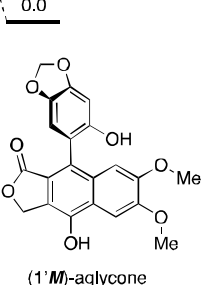

B3LYP-D3(BJ)/6-311+G(d,p)-SMD(methanol)//B3LYP/6-31G(d) 
Figure S1. Scan the rotation barrier of the 6'-hydroxyl substituted in 6'-hydroxyl justicidin $\mathrm{B}$ and the aglycone of justatropmers.

The rotation barriers of dihedral $\mathrm{C} 1-\mathrm{C} 2-\mathrm{C} 3-\mathrm{C} 4$ in the 6 '-hydroxyl justicidin $\mathrm{B}(\mathrm{R}=\mathrm{H})$ and aglycone of justatropmer $(\mathrm{R}=\mathrm{OH})$ were separately scanned at B3LYP/6-31G(d) including empirical dispersion correction in methanol by the default PCM model. The stationary points in potential energy surface were further optimized at B3LYP/6-31G(d) including empirical dispersion correction by SMD model. A local minima structure was confirmed by a successive frequency calculation at the same level of theory. (A) Dihedral angle of C1-C2-C3-C4 in 6'-hydroxyl justicidin B (A) and aglycone of justatropmers (B). (B) Quantum mechanics calculations of the energy profiles as displayed for the torsion angels of $6^{\prime}$-hydroxyl justicidin B. The axial rotation barrier energy of $6^{\prime}$-hydroxyl justicidin B was estimated as $34.8 \mathrm{kcal} / \mathrm{mol}$. (C) Quantum mechanics calculations of the energy profiles as displayed for the torsion angels of the aglycone of justatropmers. The axial rotation barrier energy of the aglycone of justatropmers was also estimated as $34.8 \mathrm{kcal} / \mathrm{mol}$. (D) More accurate prediction of the two rotational pathways for the interconversion between the two atropisomers of 6'-hydroxyl justicidin B. The barriers were found to be $26.3 \mathrm{kcal} / \mathrm{mol}$. (E) More accurate prediction of the two rotational pathways for the interconversion between the two atropisomers of aglycone. The barriers were found to be 26.5 $\mathrm{kcal} / \mathrm{mol}$.

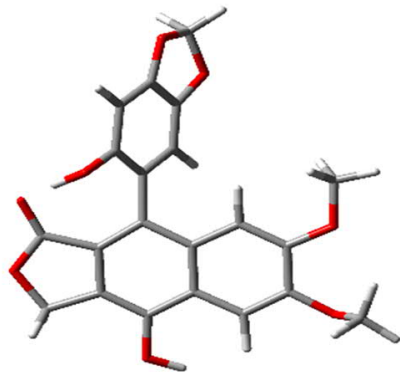

(1'P)-aglycone

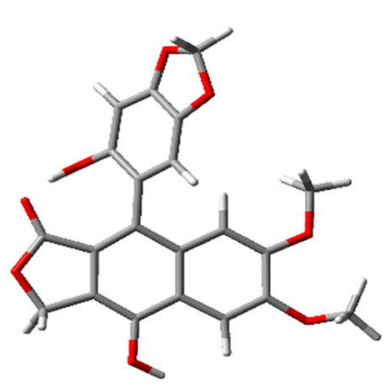

(1'M)-aglycone

Figure S2. The optimized structures of minimum energy conformer of (1'P)-aglycone and $\left(1^{\prime} \boldsymbol{M}\right)$-aglycone from the scan of the rotation barrier (no imaginary frequency was observed) 
Table S1. The Cartesian coordinates of optimized structure of (1'P)-aglycone. No imaginary frequency was observed.

\begin{tabular}{|c|c|c|c|}
\hline atom & $X$ & Y & $\mathrm{Z}$ \\
\hline $\mathrm{C}$ & -0.14174 & 2.301863 & -0.03823 \\
\hline $\mathrm{C}$ & -1.41781 & 2.830971 & -0.31058 \\
\hline $\mathrm{C}$ & -2.51168 & 2.02334 & -0.47374 \\
\hline $\mathrm{C}$ & -2.34947 & 0.618367 & -0.28931 \\
\hline $\mathrm{C}$ & -1.03841 & 0.078923 & -0.04303 \\
\hline $\mathrm{C}$ & 0.116213 & 0.937335 & 0.039028 \\
\hline $\mathrm{C}$ & -3.46006 & -0.26445 & -0.32048 \\
\hline $\mathrm{C}$ & -3.32336 & -1.62001 & -0.14645 \\
\hline $\mathrm{C}$ & -2.02066 & -2.16741 & 0.087303 \\
\hline $\mathrm{C}$ & -0.93016 & -1.32778 & 0.152496 \\
\hline $\mathrm{C}$ & 0.77455 & 3.458165 & 0.072455 \\
\hline $\mathrm{O}$ & 0.087959 & 4.599734 & -0.2085 \\
\hline $\mathrm{C}$ & -1.30683 & 4.318497 & -0.45242 \\
\hline $\mathrm{O}$ & 1.946773 & 3.498076 & 0.400076 \\
\hline $\mathrm{C}$ & 1.47756 & 0.336972 & 0.113319 \\
\hline $\mathrm{C}$ & 1.856628 & -0.5634 & -0.91803 \\
\hline $\mathrm{C}$ & 3.066731 & -1.20696 & -0.81337 \\
\hline $\mathrm{C}$ & 3.914724 & -0.99554 & 0.272266 \\
\hline $\mathrm{C}$ & 3.595026 & -0.11253 & 1.27953 \\
\hline $\mathrm{C}$ & 2.364488 & 0.570065 & 1.183723 \\
\hline $\mathrm{O}$ & -3.70774 & 2.614671 & -0.77504 \\
\hline $\mathrm{O}$ & 3.624553 & -2.14456 & -1.6601 \\
\hline $\mathrm{C}$ & 4.962687 & -2.31812 & -1.1845 \\
\hline $\mathrm{O}$ & 5.033791 & -1.78244 & 0.14417 \\
\hline $\mathrm{O}$ & 2.061794 & 1.449733 & 2.186485 \\
\hline $\mathrm{O}$ & -1.97025 & -3.51934 & 0.23236 \\
\hline $\mathrm{C}$ & -0.69633 & -4.12287 & 0.43356 \\
\hline $\mathrm{O}$ & -4.43187 & -2.41301 & -0.24686 \\
\hline $\mathrm{C}$ & -4.82131 & -3.11598 & 0.94165 \\
\hline $\mathrm{H}$ & -4.47417 & 0.102295 & -0.45248 \\
\hline $\mathrm{H}$ & 0.047361 & -1.73454 & 0.365407 \\
\hline $\mathrm{H}$ & -1.56766 & 4.675809 & -1.45489 \\
\hline $\mathrm{H}$ & -1.89908 & 4.877998 & 0.280058 \\
\hline $\mathrm{H}$ & 1.196599 & -0.74711 & -1.75839 \\
\hline $\mathrm{H}$ & 4.252439 & 0.085231 & 2.11723 \\
\hline $\mathrm{H}$ & -4.32845 & 1.950517 & -1.11056 \\
\hline $\mathrm{H}$ & 5.661176 & -1.76974 & -1.83451 \\
\hline $\mathrm{H}$ & 5.204132 & -3.38398 & -1.15836 \\
\hline $\mathrm{H}$ & 2.038835 & 2.336764 & 1.763327 \\
\hline $\mathrm{H}$ & -0.88265 & -5.1967 & 0.484302 \\
\hline
\end{tabular}




\begin{tabular}{llll}
$\mathrm{H}$ & -0.01687 & -3.9055 & -0.40045 \\
\hline $\mathrm{H}$ & -0.23659 & -3.78504 & 1.371378 \\
\hline $\mathrm{H}$ & -5.74567 & -3.63864 & 0.687292 \\
\hline $\mathrm{H}$ & -4.05973 & -3.83752 & 1.249023 \\
\hline $\mathrm{H}$ & -5.01465 & -2.40946 & 1.759736 \\
\hline
\end{tabular}

Table S2. The Cartesian coordinates of optimized structure of (1'M)-aglycone. No imaginary frequency was observed.

\begin{tabular}{|c|c|c|c|}
\hline atom & $X$ & $\mathrm{Y}$ & $\mathrm{Z}$ \\
\hline $\mathrm{C}$ & 0.130296 & 2.318066 & -0.089098 \\
\hline $\mathrm{C}$ & 1.419922 & 2.849689 & -0.273983 \\
\hline $\mathrm{C}$ & 2.53486 & 2.053352 & -0.284533 \\
\hline $\mathrm{C}$ & 2.367979 & 0.651551 & -0.081208 \\
\hline $\mathrm{C}$ & 1.047799 & 0.113498 & 0.116686 \\
\hline $\mathrm{C}$ & -0.118025 & 0.957932 & 0.064737 \\
\hline $\mathrm{C}$ & 3.474774 & -0.237289 & -0.08344 \\
\hline $\mathrm{C}$ & 3.336551 & -1.581599 & 0.160944 \\
\hline $\mathrm{C}$ & 2.035307 & -2.110812 & 0.44249 \\
\hline $\mathrm{C}$ & 0.939129 & -1.277804 & 0.404282 \\
\hline $\mathrm{C}$ & -0.801437 & 3.467249 & -0.107335 \\
\hline $\mathrm{O}$ & -0.101651 & 4.604217 & -0.374933 \\
\hline $\mathrm{C}$ & 1.311284 & 4.329475 & -0.481993 \\
\hline $\mathrm{O}$ & -1.998044 & 3.507657 & 0.115223 \\
\hline $\mathrm{C}$ & -1.474036 & 0.341626 & 0.087873 \\
\hline $\mathrm{C}$ & -1.768723 & -0.636225 & -0.899689 \\
\hline $\mathrm{C}$ & -2.976002 & -1.290084 & -0.834541 \\
\hline $\mathrm{C}$ & -3.901509 & -1.01596 & 0.170717 \\
\hline $\mathrm{C}$ & -3.663957 & -0.058999 & 1.132146 \\
\hline $\mathrm{C}$ & -2.437538 & 0.635046 & 1.074088 \\
\hline $\mathrm{O}$ & 3.743917 & 2.653576 & -0.500545 \\
\hline $\mathrm{O}$ & -3.461697 & -2.294406 & -1.648797 \\
\hline $\mathrm{C}$ & -4.828523 & -2.454501 & -1.257633 \\
\hline $\mathrm{O}$ & -4.999849 & -1.82782 & 0.021096 \\
\hline $\mathrm{O}$ & -2.216912 & 1.587781 & 2.030572 \\
\hline $\mathrm{O}$ & 1.995862 & -3.435285 & 0.751916 \\
\hline $\mathrm{C}$ & 0.730709 & -4.012254 & 1.058677 \\
\hline $\mathrm{O}$ & 4.458485 & -2.360375 & 0.204037 \\
\hline $\mathrm{C}$ & 4.540701 & -3.41492 & -0.764654 \\
\hline $\mathrm{H}$ & 4.484804 & 0.107085 & -0.288681 \\
\hline $\mathrm{H}$ & -0.04213 & -1.671537 & 0.624896 \\
\hline $\mathrm{H}$ & 1.83381 & 4.920301 & 0.278703 \\
\hline $\mathrm{H}$ & 1.656181 & 4.653778 & -1.470021 \\
\hline $\mathrm{H}$ & -1.048381 & -0.868552 & -1.675914 \\
\hline
\end{tabular}




\begin{tabular}{llll}
$\mathrm{H}$ & -4.381284 & 0.186577 & 1.905564 \\
\hline $\mathrm{H}$ & 4.463498 & 2.043687 & -0.280275 \\
\hline $\mathrm{H}$ & -5.060341 & -3.51944 & -1.172679 \\
\hline $\mathrm{H}$ & -5.484407 & -1.96395 & -1.992719 \\
\hline $\mathrm{H}$ & -2.179366 & 2.443741 & 1.548964 \\
\hline $\mathrm{H}$ & 0.928513 & -5.064802 & 1.267052 \\
\hline $\mathrm{H}$ & 0.28282 & -3.540832 & 1.942692 \\
\hline $\mathrm{H}$ & 0.035914 & -3.927809 & 0.212928 \\
\hline $\mathrm{H}$ & 5.518355 & -3.877926 & -0.616005 \\
\hline $\mathrm{H}$ & 3.75248 & -4.157921 & -0.617587 \\
\hline $\mathrm{H}$ & 4.481281 & -3.007377 & -1.782679 \\
\hline
\end{tabular}

Table S3. Relative free energies ${ }^{\mathrm{a}}$ and equilibrium populations ${ }^{\mathrm{b}}$ of conformers of $\left(1^{\prime} P\right)-9^{c}$

\begin{tabular}{ccc}
\hline Conformer & $\Delta G$ & $\mathrm{P}(\%)$ \\
\hline$(1 ' P)-9 a$ & 0.00 & 48.75 \\
$(1 ' P)-9 b$ & 0.06 & 43.93 \\
$(1 ' P)-9 c$ & 1.12 & 7.32 \\
\hline
\end{tabular}

${ }^{\mathrm{a} B} 3 \mathrm{LYP} / 6-31 \mathrm{G}(\mathrm{d})$, in $\mathrm{kcal} / \mathrm{mol}$. ${ }^{\mathrm{b}}$ From $\Delta G$ values at $298.15 \mathrm{~K}$. ${ }^{\mathrm{c}}$ in $\mathrm{MeOH}$, no imaginary frequency.

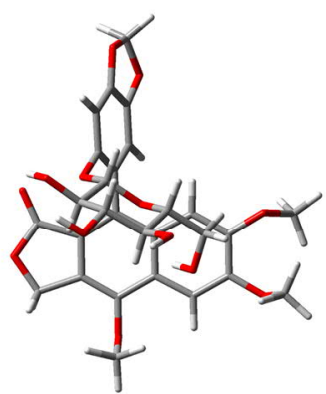

(1'P)-9a

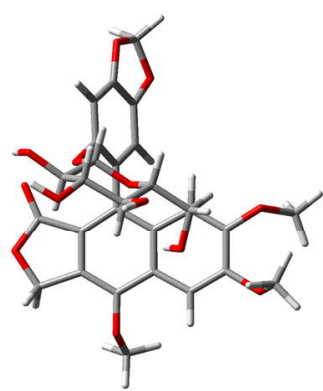

$(1 ' P)-9 b$

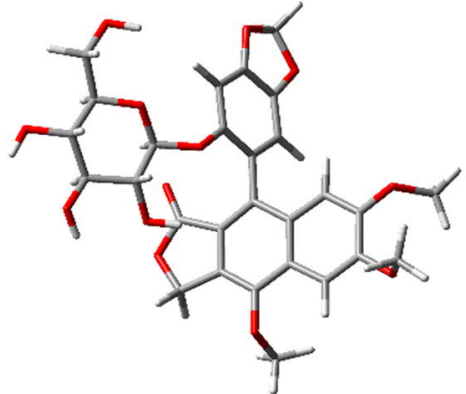

$(1 ' P)-9 c$

Figure S3. Low-energy conformers of (1'P)-9. 

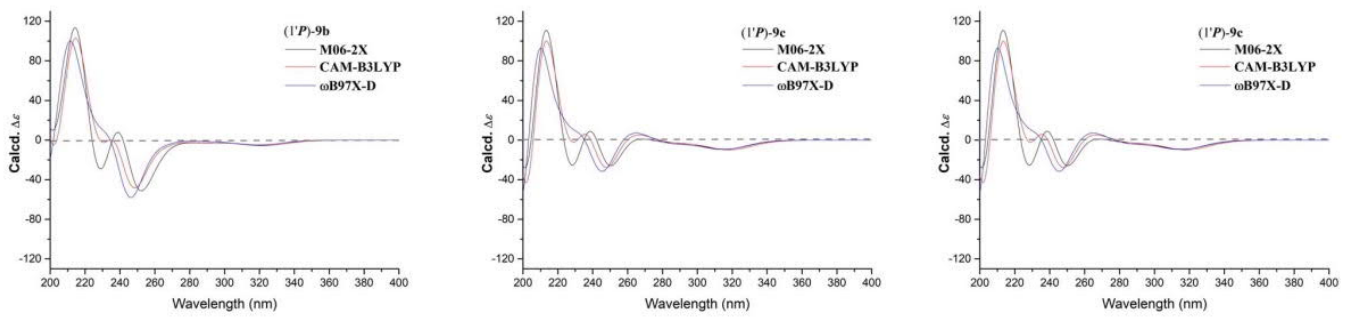

Figure S4. Calculated CD spectra of conformers of (1'P)-9 in $\mathrm{MeOH}$ using M06-2X, CAM-B3LYP and $\omega$ B97X-D functionals with def2TZVP basis sets. $\sigma=0.22 \mathrm{eV}$.

Table S4. The Cartesian coordinates of optimized structure of (1'P)-9a.

No imaginary frequency was observed.

\begin{tabular}{llll} 
atom & $\mathrm{X}$ & $\mathrm{Y}$ & $\mathrm{Z}$ \\
\hline $\mathrm{C}$ & 3.135357 & -2.254282 & -0.276501 \\
\hline $\mathrm{C}$ & 1.933481 & -2.426368 & -1.033298 \\
\hline $\mathrm{C}$ & 0.73328 & -1.987038 & -0.527295 \\
\hline $\mathrm{C}$ & 0.641499 & -1.357298 & 0.74346 \\
\hline $\mathrm{C}$ & 1.854237 & -1.200078 & 1.505216 \\
\hline $\mathrm{C}$ & 3.077508 & -1.677625 & 0.97124 \\
\hline $\mathrm{C}$ & -0.59775 & -0.848278 & 1.246807 \\
\hline $\mathrm{C}$ & -0.561521 & -0.203781 & 2.470812 \\
\hline $\mathrm{C}$ & 0.623842 & -0.027197 & 3.209852 \\
\hline $\mathrm{C}$ & 1.822566 & -0.509939 & 2.754589 \\
\hline $\mathrm{C}$ & -1.662887 & 0.415792 & 3.242486 \\
\hline $\mathrm{O}$ & -1.133472 & 0.942871 & 4.392899 \\
\hline $\mathrm{C}$ & 0.294145 & 0.72071 & 4.468537 \\
\hline $\mathrm{O}$ & 2.980475 & -0.344517 & 3.479857 \\
\hline $\mathrm{C}$ & -1.856409 & -0.979093 & 0.466395 \\
\hline $\mathrm{C}$ & -2.45564 & 0.153458 & -0.095604 \\
\hline $\mathrm{C}$ & -3.641599 & 0.087411 & -0.846163 \\
\hline $\mathrm{C}$ & -4.195991 & -1.165411 & -1.013902 \\
\hline $\mathrm{C}$ & -3.614258 & -2.306687 & -0.463151 \\
\hline $\mathrm{C}$ & -2.453175 & -2.246949 & 0.277886 \\
\hline $\mathrm{O}$ & -5.324738 & -1.507844 & -1.71008 \\
\hline $\mathrm{C}$ & -5.544941 & -2.902758 & -1.42816 \\
\hline $\mathrm{O}$ & -4.357911 & -3.412338 & -0.799271 \\
\hline $\mathrm{O}$ & -1.881231 & 1.396481 & 0.137977 \\
\hline $\mathrm{C}$ & -1.198957 & 1.979076 & -0.943341 \\
\hline $\mathrm{C}$ & 0.012199 & 1.271523 & -1.154193 \\
\hline $\mathrm{C}$ & 0.795847 & 1.803228 & -2.234185 \\
\hline & -0.179919 & 3.254723 & -1.920901 \\
\hline-0.912862 & 3.427422 & -0.551477 \\
\hline & & & \\
\hline $\mathrm{C}$ & & &
\end{tabular}




\begin{tabular}{|c|c|c|c|}
\hline $\mathrm{O}$ & -2.109518 & 4.182077 & -0.424927 \\
\hline $\mathrm{O}$ & 0.365692 & 5.385731 & -1.259773 \\
\hline $\mathrm{O}$ & 1.886802 & 3.771407 & -3.041761 \\
\hline $\mathrm{C}$ & 2.009179 & 0.891589 & -2.380835 \\
\hline $\mathrm{O}$ & 2.807949 & 0.866062 & -1.202471 \\
\hline $\mathrm{O}$ & -2.84824 & 0.49374 & 3.005357 \\
\hline $\mathrm{C}$ & 3.685849 & 0.873261 & 3.173451 \\
\hline $\mathrm{O}$ & 4.350687 & -2.653328 & -0.767214 \\
\hline $\mathrm{C}$ & 4.803653 & -1.914676 & -1.923141 \\
\hline $\mathrm{O}$ & 1.959583 & -2.955064 & -2.300284 \\
\hline $\mathrm{C}$ & 2.342933 & -4.340572 & -2.379114 \\
\hline $\mathrm{H}$ & -0.152882 & -2.111423 & -1.137294 \\
\hline $\mathrm{H}$ & 3.99594 & -1.550357 & 1.532078 \\
\hline $\mathrm{H}$ & 0.788381 & 1.694891 & 4.540606 \\
\hline $\mathrm{H}$ & 0.509472 & 0.150301 & 5.377606 \\
\hline $\mathrm{H}$ & -4.091137 & 0.983524 & -1.257787 \\
\hline $\mathrm{H}$ & -2.007403 & -3.1388 & 0.70357 \\
\hline $\mathrm{H}$ & -6.39622 & -3.003838 & -0.743243 \\
\hline $\mathrm{H}$ & -5.719429 & -3.437662 & -2.364057 \\
\hline $\mathrm{H}$ & -1.816708 & 1.940779 & -1.855683 \\
\hline $\mathrm{H}$ & 0.217232 & 1.78071 & -3.171561 \\
\hline $\mathrm{H}$ & 1.813899 & 3.252775 & -1.022402 \\
\hline $\mathrm{H}$ & -0.668943 & 4.133905 & -2.560338 \\
\hline $\mathrm{H}$ & -0.344618 & 3.409752 & 0.390297 \\
\hline $\mathrm{H}$ & -2.622235 & 3.784098 & 0.29816 \\
\hline $\mathrm{H}$ & -0.433607 & 5.91193 & -1.091857 \\
\hline $\mathrm{H}$ & 1.992164 & 4.723915 & -2.878419 \\
\hline $\mathrm{H}$ & 2.642561 & 1.275114 & -3.184289 \\
\hline $\mathrm{H}$ & 1.66973 & -0.117579 & -2.650039 \\
\hline $\mathrm{H}$ & 2.244488 & 0.517844 & -0.491544 \\
\hline $\mathrm{H}$ & 3.955214 & 0.911679 & 2.111267 \\
\hline $\mathrm{H}$ & 3.078953 & 1.751267 & 3.424662 \\
\hline $\mathrm{H}$ & 4.590595 & 0.865202 & 3.783498 \\
\hline $\mathrm{H}$ & 4.731091 & -0.839635 & -1.73014 \\
\hline $\mathrm{H}$ & 5.844117 & -2.206961 & -2.074867 \\
\hline $\mathrm{H}$ & 4.21109 & -2.165734 & -2.807474 \\
\hline $\mathrm{H}$ & 2.298471 & -4.604924 & -3.437023 \\
\hline $\mathrm{H}$ & 3.357151 & -4.493781 & -1.999151 \\
\hline $\mathrm{H}$ & 1.637914 & -4.961824 & -1.813812 \\
\hline
\end{tabular}


Table S5. The Cartesian coordinates of optimized structure of $\left(1^{\prime} \boldsymbol{P}\right)-\mathbf{9 b}$. No imaginary frequency was observed.

\begin{tabular}{|c|c|c|c|}
\hline atom & $X$ & $\mathrm{Y}$ & $\mathrm{Z}$ \\
\hline $\mathrm{C}$ & -3.489917 & -1.22466 & 1.007736 \\
\hline $\mathrm{C}$ & -2.303301 & -1.331992 & 1.800565 \\
\hline $\mathrm{C}$ & -1.070208 & -1.354933 & 1.193885 \\
\hline $\mathrm{C}$ & -0.929044 & -1.292382 & -0.219093 \\
\hline $\mathrm{C}$ & -2.129504 & -1.226863 & -1.012997 \\
\hline $\mathrm{C}$ & -3.389746 & -1.192066 & -0.364595 \\
\hline $\mathrm{C}$ & 0.356242 & -1.253097 & -0.847317 \\
\hline $\mathrm{C}$ & 0.377789 & -1.126097 & -2.225119 \\
\hline $\mathrm{C}$ & -0.791382 & -1.066995 & -3.007363 \\
\hline $\mathrm{C}$ & -2.034171 & -1.125341 & -2.434087 \\
\hline $\mathrm{C}$ & 1.533476 & -1.044961 & -3.146847 \\
\hline $\mathrm{O}$ & 1.051564 & -0.922796 & -4.425358 \\
\hline $\mathrm{C}$ & -0.395267 & -0.928963 & -4.448154 \\
\hline $\mathrm{O}$ & -3.170708 & -1.035589 & -3.204656 \\
\hline $\mathrm{C}$ & 1.607289 & -1.324623 & -0.046534 \\
\hline $\mathrm{C}$ & 2.437507 & -0.205191 & 0.071733 \\
\hline $\mathrm{C}$ & 3.63136 & -0.224772 & 0.812776 \\
\hline $\mathrm{C}$ & 3.950853 & -1.413839 & 1.436326 \\
\hline $\mathrm{C}$ & 3.13565 & -2.540328 & 1.333275 \\
\hline $\mathrm{C}$ & 1.966416 & -2.528217 & 0.602991 \\
\hline $\mathrm{O}$ & 5.033588 & -1.693577 & 2.227479 \\
\hline $\mathrm{C}$ & 4.974579 & -3.11083 & 2.475506 \\
\hline $\mathrm{O}$ & 3.677411 & -3.57091 & 2.063648 \\
\hline $\mathrm{O}$ & 2.089021 & 0.956395 & -0.605459 \\
\hline $\mathrm{C}$ & 1.609299 & 2.012213 & 0.187907 \\
\hline $\mathrm{O}$ & 0.313288 & 1.677764 & 0.656229 \\
\hline $\mathrm{C}$ & -0.272736 & 2.702975 & 1.473752 \\
\hline $\mathrm{C}$ & -0.417696 & 3.990492 & 0.652642 \\
\hline $\mathrm{C}$ & 0.930027 & 4.40058 & 0.066868 \\
\hline $\mathrm{C}$ & 1.552994 & 3.247115 & -0.708961 \\
\hline $\mathrm{O}$ & 2.849842 & 3.65781 & -1.11788 \\
\hline $\mathrm{O}$ & 0.701139 & 5.537021 & -0.760365 \\
\hline $\mathrm{O}$ & -0.925106 & 5.002883 & 1.51335 \\
\hline $\mathrm{C}$ & -1.612494 & 2.158356 & 1.956388 \\
\hline $\mathrm{O}$ & -2.49552 & 1.874969 & 0.876412 \\
\hline $\mathrm{O}$ & 2.724185 & -1.075506 & -2.926137 \\
\hline $\mathrm{C}$ & -3.726407 & -2.309484 & -3.580191 \\
\hline $\mathrm{O}$ & -4.731352 & -1.154943 & 1.58099 \\
\hline $\mathrm{C}$ & -4.968116 & 0.014438 & 2.396095 \\
\hline $\mathrm{O}$ & -2.36199 & -1.329156 & 3.172566 \\
\hline
\end{tabular}




\begin{tabular}{llll}
$\mathrm{C}$ & -2.989114 & -2.485262 & 3.75787 \\
\hline $\mathrm{H}$ & -0.191605 & -1.397179 & 1.825701 \\
\hline $\mathrm{H}$ & -4.293668 & -1.080344 & -0.951939 \\
\hline $\mathrm{H}$ & -0.739407 & 0.006727 & -4.900113 \\
\hline $\mathrm{H}$ & -0.724775 & -1.763404 & -5.075449 \\
\hline $\mathrm{H}$ & 4.263238 & 0.653508 & 0.875128 \\
\hline $\mathrm{H}$ & 1.338483 & -3.408613 & 0.52589 \\
\hline $\mathrm{H}$ & 5.748687 & -3.614736 & 1.883051 \\
\hline $\mathrm{H}$ & 5.104594 & -3.296625 & 3.543618 \\
\hline $\mathrm{H}$ & 2.280711 & 2.187984 & 1.044587 \\
\hline $\mathrm{H}$ & 0.367225 & 2.905594 & 2.347636 \\
\hline $\mathrm{H}$ & -1.115016 & 3.790422 & -0.1737 \\
\hline $\mathrm{H}$ & 1.603924 & 4.664011 & 0.898302 \\
\hline $\mathrm{H}$ & 0.915673 & 3.001945 & -1.571306 \\
\hline $\mathrm{H}$ & 3.223536 & 2.934895 & -1.648766 \\
\hline $\mathrm{H}$ & 1.563292 & 5.799498 & -1.123363 \\
\hline $\mathrm{H}$ & -0.877895 & 5.833295 & 1.010127 \\
\hline $\mathrm{H}$ & -2.100582 & 2.917048 & 2.572785 \\
\hline $\mathrm{H}$ & -1.437716 & 1.263991 & 2.569394 \\
\hline $\mathrm{H}$ & -2.065517 & 1.191233 & 0.336065 \\
\hline $\mathrm{H}$ & -3.011796 & -2.884414 & -4.181224 \\
\hline $\mathrm{H}$ & -4.00655 & -2.891594 & -2.694544 \\
\hline $\mathrm{H}$ & -4.615816 & -2.091967 & -4.173933 \\
\hline $\mathrm{H}$ & -6.042822 & 0.030897 & 2.58539 \\
\hline $\mathrm{H}$ & -4.422246 & -0.045941 & 3.341602 \\
\hline & -2.948779 & -2.335193 & 4.838072 \\
\hline-4.029879 & -2.578685 & 3.43365 \\
\hline-2.435502 & -3.393313 & 3.490431 \\
\hline $\mathrm{H}$ & & & \\
\hline $\mathrm{H}$ & & & \\
\hline & & &
\end{tabular}

Table S6. The Cartesian coordinates of optimized structure of $\left(1^{\prime} \boldsymbol{P}\right)-9 \mathbf{c}$. No imaginary frequency was observed.

\begin{tabular}{llll} 
atom & $\mathrm{X}$ & $\mathrm{Y}$ & $\mathrm{Z}$ \\
\hline $\mathrm{C}$ & -4.729515 & -0.210385 & -1.314085 \\
\hline $\mathrm{C}$ & -3.97343 & 1.004733 & -1.372824 \\
\hline $\mathrm{C}$ & -2.791877 & 1.111918 & -0.677872 \\
\hline $\mathrm{C}$ & -2.301941 & 0.050496 & 0.13112 \\
\hline $\mathrm{C}$ & -3.086634 & -1.154495 & 0.214258 \\
\hline $\mathrm{C}$ & -4.287222 & -1.252721 & -0.53204 \\
\hline $\mathrm{C}$ & -1.068078 & 0.158322 & 0.846223 \\
\hline $\mathrm{C}$ & -0.679076 & -0.935757 & 1.600401 \\
\hline $\mathrm{C}$ & -1.438299 & -2.119221 & 1.681913 \\
\hline
\end{tabular}




\begin{tabular}{|c|c|c|c|}
\hline $\mathrm{C}$ & -2.62627 & -2.247101 & 1.009217 \\
\hline $\mathrm{C}$ & 0.530319 & -1.128049 & 2.434761 \\
\hline $\mathrm{O}$ & 0.475849 & -2.377496 & 2.985507 \\
\hline $\mathrm{C}$ & -0.699514 & -3.098399 & 2.547151 \\
\hline $\mathrm{O}$ & -3.346048 & -3.418228 & 1.062711 \\
\hline $\mathrm{C}$ & -0.225765 & 1.384341 & 0.771793 \\
\hline $\mathrm{C}$ & 0.949709 & 1.38356 & 0.011128 \\
\hline $\mathrm{C}$ & 1.81268 & 2.4928 & -0.037253 \\
\hline $\mathrm{C}$ & 1.43872 & 3.595848 & 0.703239 \\
\hline $\mathrm{C}$ & 0.258728 & 3.624663 & 1.444265 \\
\hline $\mathrm{C}$ & -0.592373 & 2.541053 & 1.494912 \\
\hline $\mathrm{O}$ & 2.082522 & 4.801014 & 0.80725 \\
\hline $\mathrm{C}$ & 1.356944 & 5.540823 & 1.806818 \\
\hline $\mathrm{O}$ & 0.116096 & 4.855309 & 2.040169 \\
\hline $\mathrm{O}$ & 1.223624 & 0.249433 & -0.74017 \\
\hline $\mathrm{C}$ & 2.450239 & -0.394379 & -0.508398 \\
\hline $\mathrm{O}$ & 3.450071 & 0.206343 & -1.326541 \\
\hline $\mathrm{C}$ & 4.750741 & -0.360654 & -1.103996 \\
\hline $\mathrm{C}$ & 4.734815 & -1.845234 & -1.487989 \\
\hline $\mathrm{C}$ & 3.621151 & -2.572953 & -0.737597 \\
\hline $\mathrm{C}$ & 2.288536 & -1.857556 & -0.926907 \\
\hline $\mathrm{O}$ & 1.321857 & -2.546909 & -0.143935 \\
\hline $\mathrm{O}$ & 3.585736 & -3.906863 & -1.23188 \\
\hline $\mathrm{O}$ & 6.00731 & -2.395803 & -1.176108 \\
\hline $\mathrm{C}$ & 5.726846 & 0.452264 & -1.944499 \\
\hline $\mathrm{O}$ & 5.744897 & 1.821446 & -1.562792 \\
\hline $\mathrm{O}$ & 1.455094 & -0.377822 & 2.665093 \\
\hline $\mathrm{C}$ & -4.330964 & -3.453906 & 2.112432 \\
\hline $\mathrm{O}$ & -5.912416 & -0.34724 & -1.993802 \\
\hline $\mathrm{C}$ & -5.804709 & -0.335424 & -3.430504 \\
\hline $\mathrm{O}$ & -4.381926 & 2.057059 & -2.154859 \\
\hline $\mathrm{C}$ & -5.588912 & 2.708173 & -1.716061 \\
\hline $\mathrm{H}$ & -2.235022 & 2.038868 & -0.752552 \\
\hline $\mathrm{H}$ & -4.861166 & -2.171892 & -0.510467 \\
\hline $\mathrm{H}$ & -0.371444 & -3.985344 & 1.995994 \\
\hline $\mathrm{H}$ & -1.256773 & -3.416561 & 3.433231 \\
\hline $\mathrm{H}$ & 2.720459 & 2.462855 & -0.62354 \\
\hline $\mathrm{H}$ & -1.503498 & 2.562324 & 2.082106 \\
\hline $\mathrm{H}$ & 1.942434 & 5.566942 & 2.734622 \\
\hline $\mathrm{H}$ & 1.152981 & 6.546929 & 1.434693 \\
\hline $\mathrm{H}$ & 2.723453 & -0.310774 & 0.552357 \\
\hline $\mathrm{H}$ & 5.021047 & -0.262365 & -0.04129 \\
\hline $\mathrm{H}$ & 4.525274 & -1.927019 & -2.566956 \\
\hline
\end{tabular}




\begin{tabular}{llll}
$\mathrm{H}$ & 3.866752 & -2.569588 & 0.336869 \\
\hline $\mathrm{H}$ & 2.015263 & -1.869915 & -1.991609 \\
\hline $\mathrm{H}$ & 0.45507 & -2.162719 & -0.353316 \\
\hline $\mathrm{H}$ & 2.870129 & -4.362395 & -0.757847 \\
\hline $\mathrm{H}$ & 5.924383 & -3.357036 & -1.296571 \\
\hline $\mathrm{H}$ & 5.466401 & 0.334123 & -3.007841 \\
\hline $\mathrm{H}$ & 6.735735 & 0.060824 & -1.793262 \\
\hline $\mathrm{H}$ & 4.852595 & 2.163739 & -1.732528 \\
\hline $\mathrm{H}$ & -3.85722 & -3.357147 & 3.096594 \\
\hline $\mathrm{H}$ & -5.067983 & -2.652652 & 1.984034 \\
\hline $\mathrm{H}$ & -4.8243 & -4.424192 & 2.035554 \\
\hline $\mathrm{H}$ & -5.178136 & -1.169072 & -3.769361 \\
\hline $\mathrm{H}$ & -6.820809 & -0.463066 & -3.807455 \\
\hline $\mathrm{H}$ & -5.38898 & 0.609698 & -3.790119 \\
\hline $\mathrm{H}$ & -6.435636 & 2.015645 & -1.71533 \\
\hline $\mathrm{H}$ & -5.451014 & 3.125579 & -0.711348 \\
\hline $\mathrm{H}$ & -5.770417 & 3.515709 & -2.42741 \\
\hline & & &
\end{tabular}




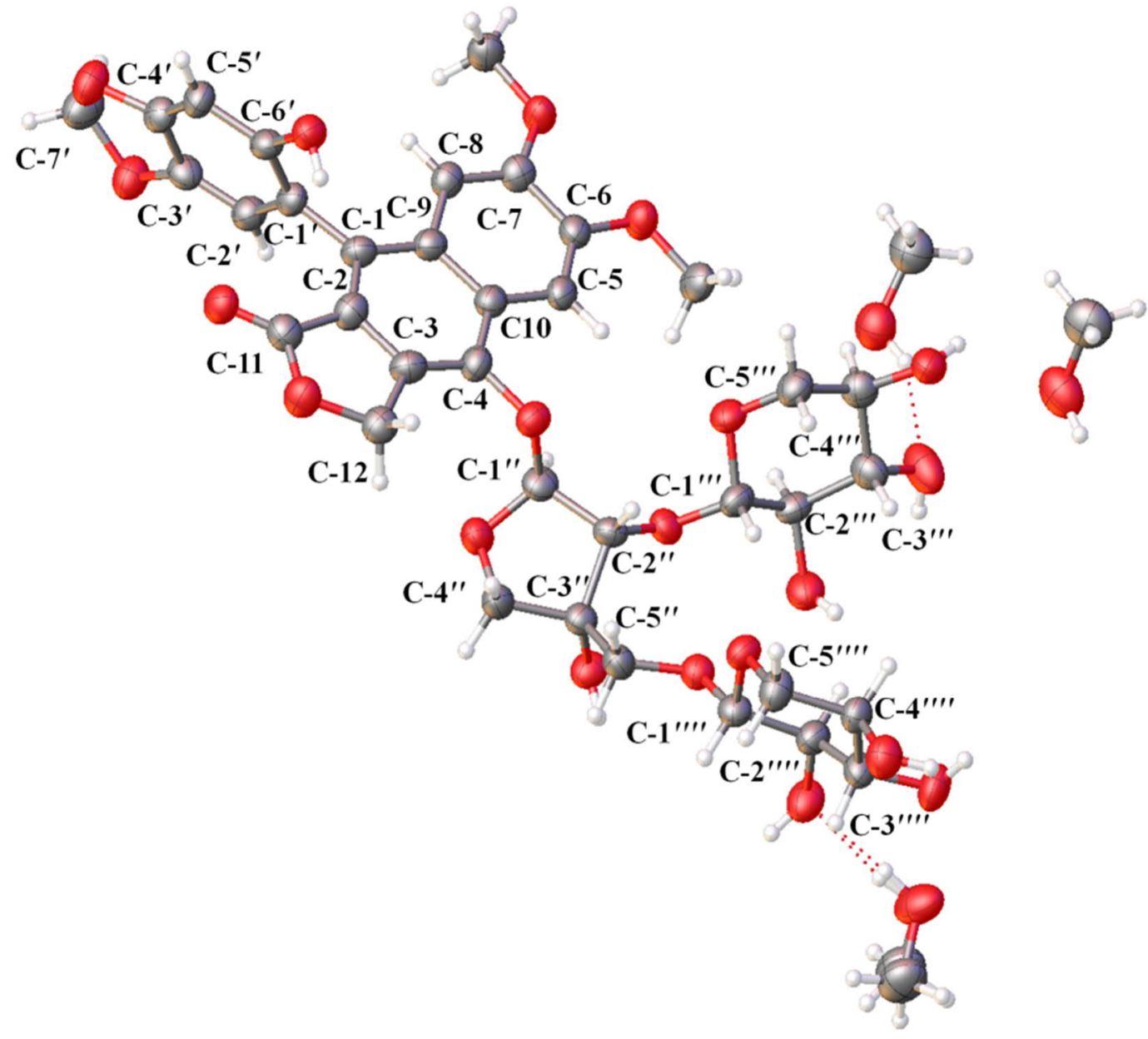

Figure S5. ORTEP view of $\mathbf{5}$ showing thermal ellipsoids at the 50\% probability level. 
Table S7. Crystal Data of Compound 5.

\begin{tabular}{|c|c|}
\hline Identification code & cxy0762 \\
\hline Empirical formula & $\mathrm{C}_{38} \mathrm{H}_{48} \mathrm{O}_{22}$ \\
\hline Formula weight & 856.76 \\
\hline Temperature/K & 100.0 \\
\hline Crystal system & orthorhombic \\
\hline Space group & $\mathrm{P} 2{ }_{1} 2{ }_{1}$ \\
\hline $\mathrm{a} / \AA$ & $15.7925(11)$ \\
\hline $\mathrm{b} / \AA$ & $23.0140(16)$ \\
\hline $\mathrm{c} / \AA$ & $12.3780(8)$ \\
\hline$\alpha /^{\circ}$ & 90 \\
\hline$\beta /^{\circ}$ & 90 \\
\hline$\gamma /{ }^{\circ}$ & 90 \\
\hline Volume $/ \AA^{3}$ & $4498.8(5)$ \\
\hline $\mathrm{Z}$ & 4 \\
\hline$\rho_{\text {calc }} \mathrm{g} / \mathrm{cm}^{3}$ & 1.265 \\
\hline$\mu / \mathrm{mm}^{-1}$ & 0.901 \\
\hline $\mathrm{F}(000)$ & 1808.0 \\
\hline Crystal size $/ \mathrm{mm}^{3}$ & $0.38 \times 0.38 \times 0.26$ \\
\hline Radiation & $\mathrm{CuK} \alpha(\lambda=1.54178)$ \\
\hline $2 \Theta$ range for data collection $/^{\circ}$ & 6.788 to 137.182 \\
\hline Index ranges & $-15 \leqslant \mathrm{~h} \leqslant 18,-27 \leqslant \mathrm{k} \leqslant 23,-14 \leqslant 1 \leqslant 12$ \\
\hline Reflections collected & 20308 \\
\hline Independent reflections & $8033\left[\mathrm{R}_{\text {int }}=0.0530, \mathrm{R}_{\text {sigma }}=0.0568\right]$ \\
\hline Data/restraints/parameters & $8033 / 32 / 580$ \\
\hline Goodness-of-fit on $\mathrm{F}^{2}$ & 1.048 \\
\hline Final $R$ indexes $[I>=2 \sigma(I)]$ & $\mathrm{R}_{1}=0.0874, \mathrm{wR}_{2}=0.2396$ \\
\hline Final $\mathrm{R}$ indexes [all data] & $\mathrm{R}_{1}=0.0983, \mathrm{wR}_{2}=0.2516$ \\
\hline Largest diff. peak/hole / e $\AA^{-3}$ & $0.75 /-0.38$ \\
\hline Flack parameter & $0.20(19)$ \\
\hline
\end{tabular}

Following the purification by recrystallization, compound $\mathbf{5}$ was dissolved in a small volume of $\mathrm{MeOH}$. After slow evaporation of the solvent at room temperature, the yellowish crystals of compound 5 were formed with 2 equivalents of $\mathrm{MeOH}$. A suitable crystal was selected and measured on a Bruker APEX-II CCD diffractometer. The crystal was kept at $100.0 \mathrm{~K}$ during data collection. Using Olex $2,{ }^{2}$ the structure was solved with the ShelXT ${ }^{3}$ structure solution program using Intrinsic Phasing and refined with the ShelX ${ }^{4}$ refinement package using Least Squares minimization. The details of the X-ray diffraction measurements and the crystal parameters are given in Table S7. The Crystallographic data for the structure of $\mathbf{5}$ has been deposited in Cambridge Crystallographic Data Center [deposition numbers: CCDC 1974784]. Copies of the data can be obtained free of charge from the CCDC via www.ccdc.cam.ac.uk. 


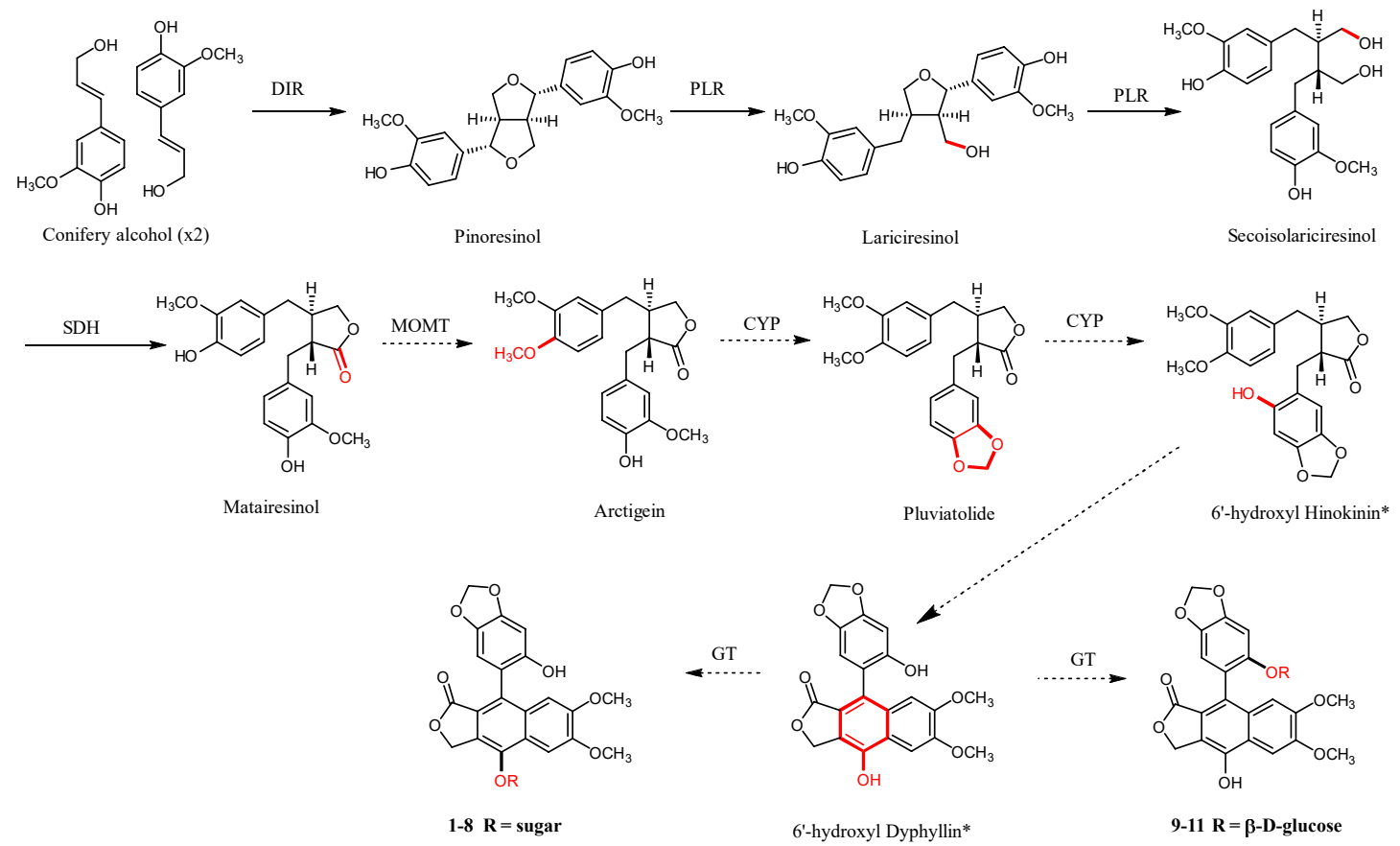

Figure S6. Plausible Biosynthetic Pathway of atropisomers 1-11.

Solid and broken lines represent the identified and unidentified enzyme-catalyzed reactions, respectively. $*$ Represent the intermediates unidentified until now.

They are all origin from conifer alcohol derived from the shikimic acid biosynthetic pathway, ${ }^{5-7}$ two coniferyl alcohol units with the aid of a dirigent protein (DIR) to give rise to pinoresinol, the pinoresinol is then reduced to ariciresinol and secoisolariciresinol with the aid of pinoresinol/lariciresinol reductase (PLR) in sequential, ${ }^{8}$ the secoisolariciresinol was then convert into matairesinol under the secoisolariciresinol dehydrogenase ( $\mathrm{SDH}),{ }^{9}$ the matairrsinol is believed to be a central intermediate leading to all diverse lignans, ${ }^{10-12}$ matairesinol is metabolized to arctigenin by matairesinol O-methyltransferase (MOMT) via methylation of a phenolic hydroxyl group, ${ }^{13,14}$ and then form into the pluviatolide and $6^{\prime}$-hydroxyl hinokinin under the enzyme of cytochrome p450 (CYP) in sequential, ${ }^{15} 6^{\prime}$-hydroxyl hinokinin is converted via cyclisation and hydroxylate ${ }^{16}$ to $6^{\prime}$-hydroxyl diphyllin, and then undergoes various glucosylation glucosyltranferases (GT), produced two class of atropisomers, one is 4-O-substituted sugar atropisomers $\mathbf{1 - 8}$, anther is $6^{\prime}$ - $O$-substituted atropisomers 9-11. 


\section{References}

(1) Frisch, M. J.; Trucks, G. W.; Schlegel, H. B.; Scuseria, G. E.; Robb, M. A.; Cheeseman, J. R.; Scalmani, G.; Barone, V.; Petersson, G. A.; Nakatsuji, H.; Li, X.; Caricato, M.; Marenich, A. V.; Bloino, J.; Janesko, B. G.; Gomperts, R.; Mennucci, B.; Hratchian, H. P.; Ortiz, J. V.; Izmaylov, A. F.; Sonnenberg, J. L.; Williams-Young, D.; Ding, F.; Lipparini, F.; Egidi, F.; Goings, J.; Peng, B.; Petrone, A.; Henderson, T.; Ranasinghe, D.; Zakrzewski, V. G.; Gao, J.; Rega, N.; Zheng, G.; Liang, W.; Hada, M.; Ehara, M.; Toyota, K.; Fukuda, R.; Hasegawa, J.; Ishida, M.; Nakajima, T.; Honda, Y.; Kitao, O.; Nakai, H.; Vreven, T.; Throssell, K.; Montgomery, J. A., Jr.; Peralta, J. E.; Ogliaro, F.; Bearpark, M. J.; Heyd, J. J.; Brothers, E. N.; Kudin, K. N.; Staroverov, V. N.; Keith, T. A.; Kobayashi, R.; Normand, J.; Raghavachari, K.; Rendell, A. P.; Burant, J. C.; Iyengar, S. S.; Tomasi, J.; Cossi, M.; Millam, J. M.; Klene, M.; Adamo, C.; Cammi, R.; Ochterski, J. W.; Martin, R. L.; Morokuma, K.;

Farkas, O.; Foresman, J. B.; Fox, D. J. Gaussian 16, revision C.01, Gaussian, Inc.: Wallingford, CT, USA, 2016.

(2) Dolomanov, O. V.; Bourhis, L. J.; Gildea,R. J; Howard, J. A. K; Puschmann, H. A Complete Structure Solution, Refinement and Analysis Program. J. Appl. Cryst., 2009, 42, 339-341.

(3) Sheldrick, G. M. Integrated space-group and crystal-structure determination. Acta Cryst. 2015, A71, 3-8.

(4) Sheldrick, G. M. Crystal Structure Refinement with SHELXL. Acta Cryst. 2015, C71, 3-8.

(5) Umezawa, T.; Davin, L. B.; Lewis N. G. Formation of lignans (-)-secoisolariciresinol and (-)-matairesinol with Forsythia intermedia cell-free extracts. J. Biol. Chem. 1991, 266, 10210-10217.

(6) Vogt, T. Phenylpropanoid biosynthesis. Mol. Plant, 2010, 3, 2-20.

(7) Dar, A. A.; Arumugam, N. Lignans of sesame: Purification methods, biological activities and biosynthesis-A review. Bioorg. Chem., 2013, 50, 1-10.

(8) Hemmati, S. T.; Schmidt, J.; Fuss, E. (+)-Pinoresinol/(-)-lariciresinol reductase 
from Linum perenne Himmelszelt involved in the biosynthesis of justicidin B. Febs. Lett., 2007, 581, 603-610.

(9) Davin, L. B.; Lewis, N. G. An historical perspective on lignan biosynthesis: Monolignol, allylphenol and hydroxycinnamic acid coupling and downstream metabolism. Phytochem. Rev., 2003, 2, 257-288.

(10) Schmidt, T. J.; Hemmati, S.; Klaes M., ; Konuklugil, B.; Mohagheghzadeh, A.; Ionkova, I.; Fuss, E.; Wilhelm, A. A. Lignans in flowering aerial parts of Linum species-Chemodiversity in the light of systematics and phylogeny. Phytochemistry. 2010, 71, 1714-1728.

(11) Satake, H.; Ono, E.; Murata, J. Recent Advances in the metabolic engineering of lignan biosynthesis pathways for the production of transgenic plant-based foods and supplements. J. Agric. Food Chem. 2013, 61, 11721-11729.

(12) Satake, H.; Koyama, T.; Bahabadi, S. E.; Matsumoto, E.; Ono, E.; Murata, J. Essences in metabolic engineering of lignan biosynthesis. Metabolites, 2015, 5, 270-290.

(13) Umezawa, T.; Ragamustari, S. K.; Nakatsubo, T.; Wada, S.; Li, L.; Yamamura, M.; Sakakibara, N.; Hattori, T.; Suzuki, S.; Chiang, V. L. A lignan O-methyltransferase catalyzing the regioselective methylation of matairesinol in Carthamus tinctorius. Plant Biotechnol. 2013, 30, 97-109.

(14) Ragamustari, S. K.; Yamamura, M.; Ono, E; Hattori, T.; Suzuki, S.; Suzuki, H.; Shibata, D.; Umezawa, T. Substrate-enantiomer selectivity of matairesinol O-methyltransferases. Plant Biotechnol. 2014, 31, 257-267.

(15) Marques, J. V.; Kim, K. W.; Lee, C.; Costa, M. A.; May, G. D.; Crow, J. A.; Davin, L. B.; Lewis, N. G. Next generation sequencing in predicting gene function in podophyllotoxin biosynthesis J. Biol. Chem. 2013, 288, 466-479.

(16) Hemmati, S.; Schneider, B.; Schmidt, T. J.; Federolf, K.; Alfermann, A.W.; Fuss, E. Justicidin B 7-hydroxylase, a cytochrome P450 monooxygenase from cell cultures of Linum perenne Himmelszelt involved in the biosynthesis of diphyllin. Phytochemistry, 2007, 68, 2736-2743. 


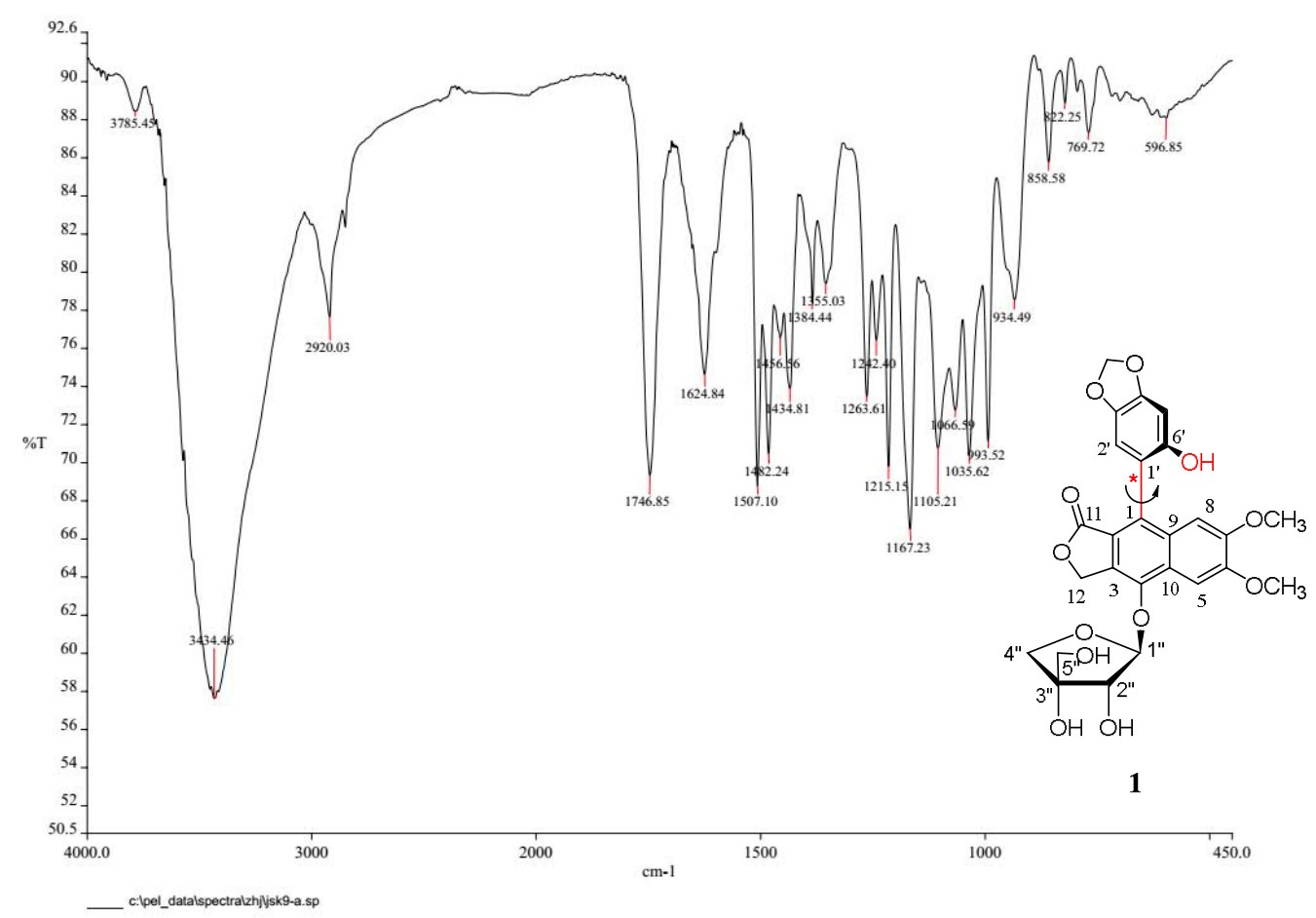

Figure S7. The IR Spectrum of Compound 1.

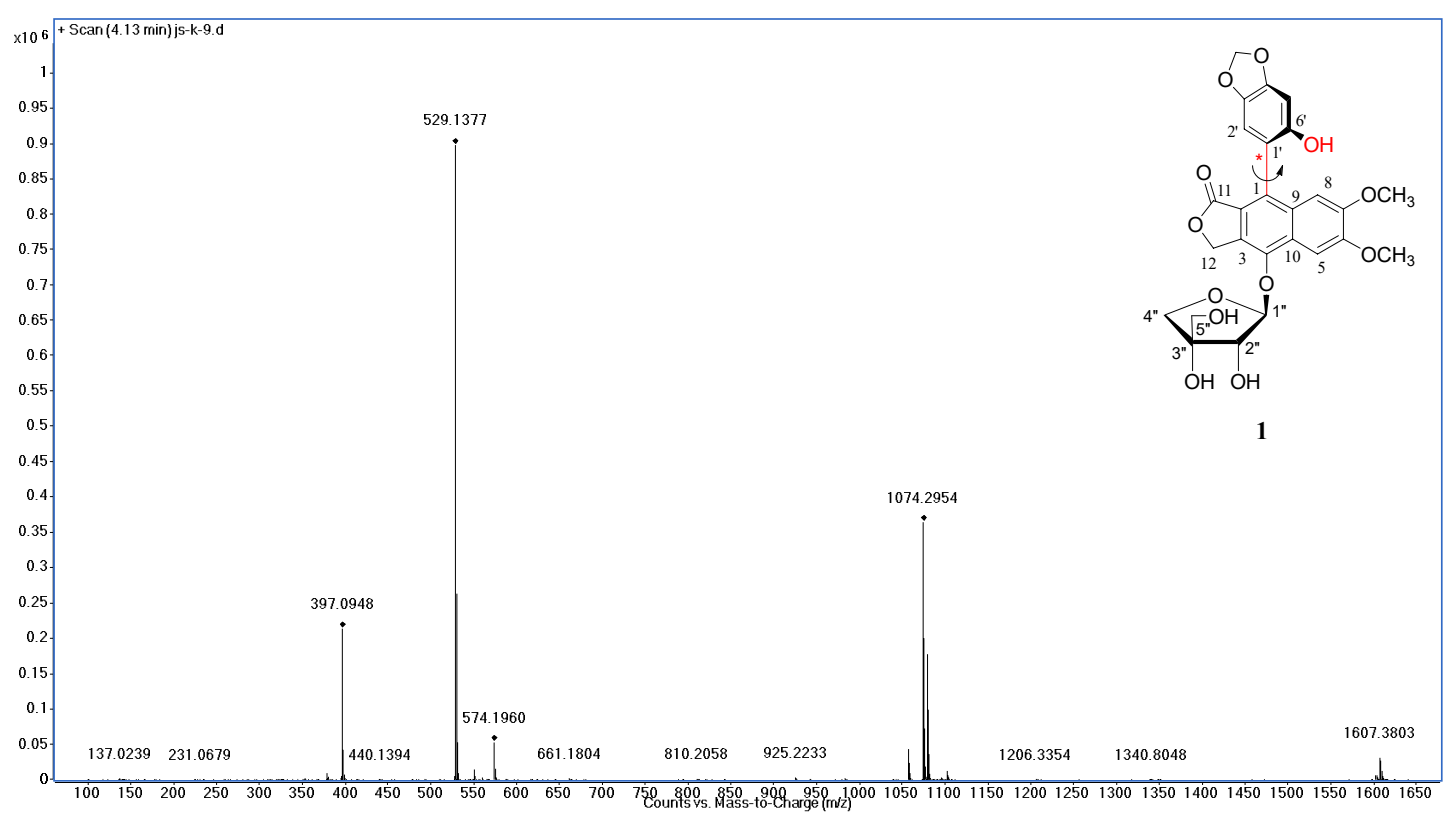

Figure S8. HRESIMS Data of Compound 1. 


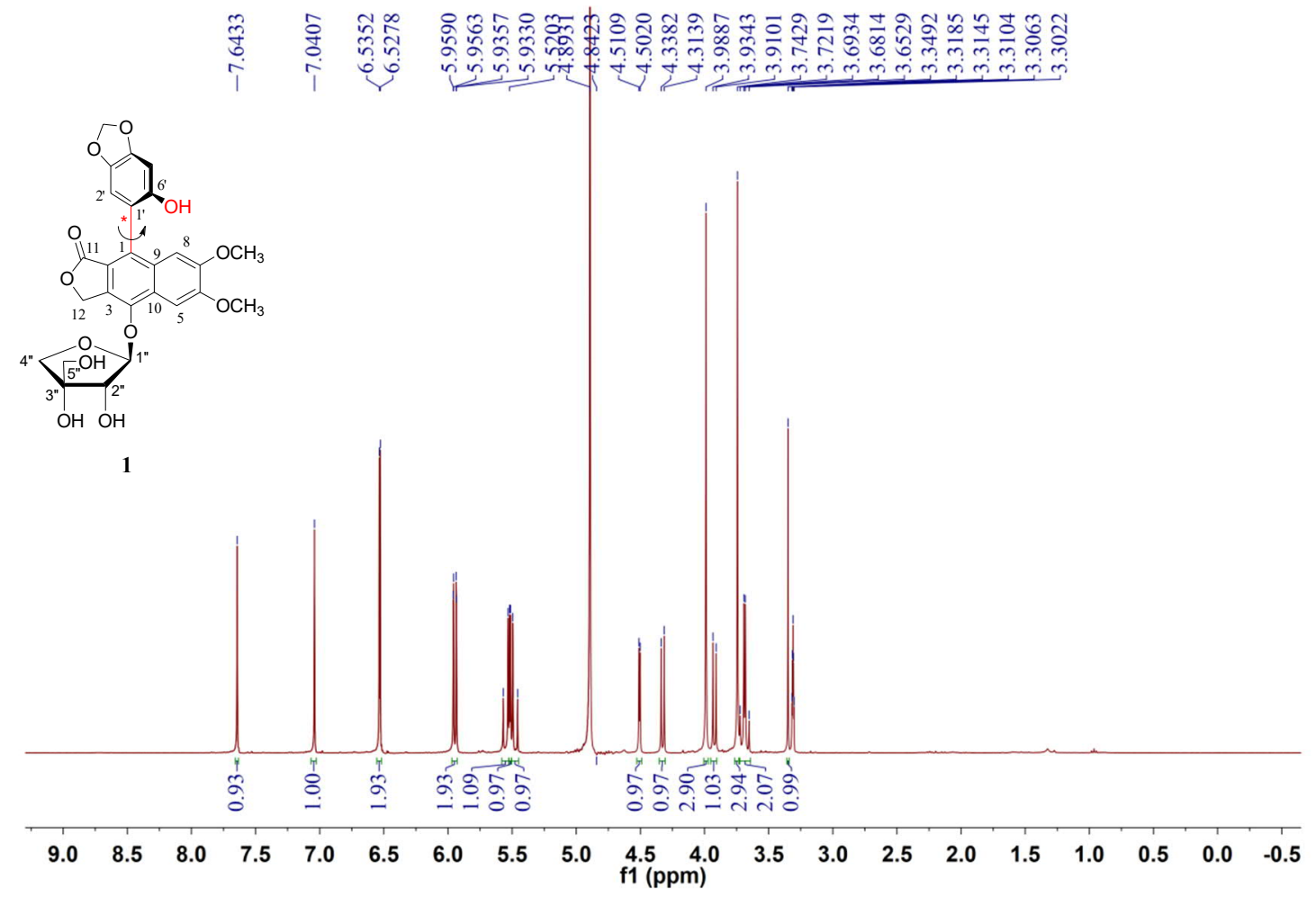

Figure S9. The ${ }^{1} \mathrm{H}$ NMR Spectrum of Compound 1 in $\mathrm{CD}_{3} \mathrm{OD}(400 \mathrm{MHz})$.

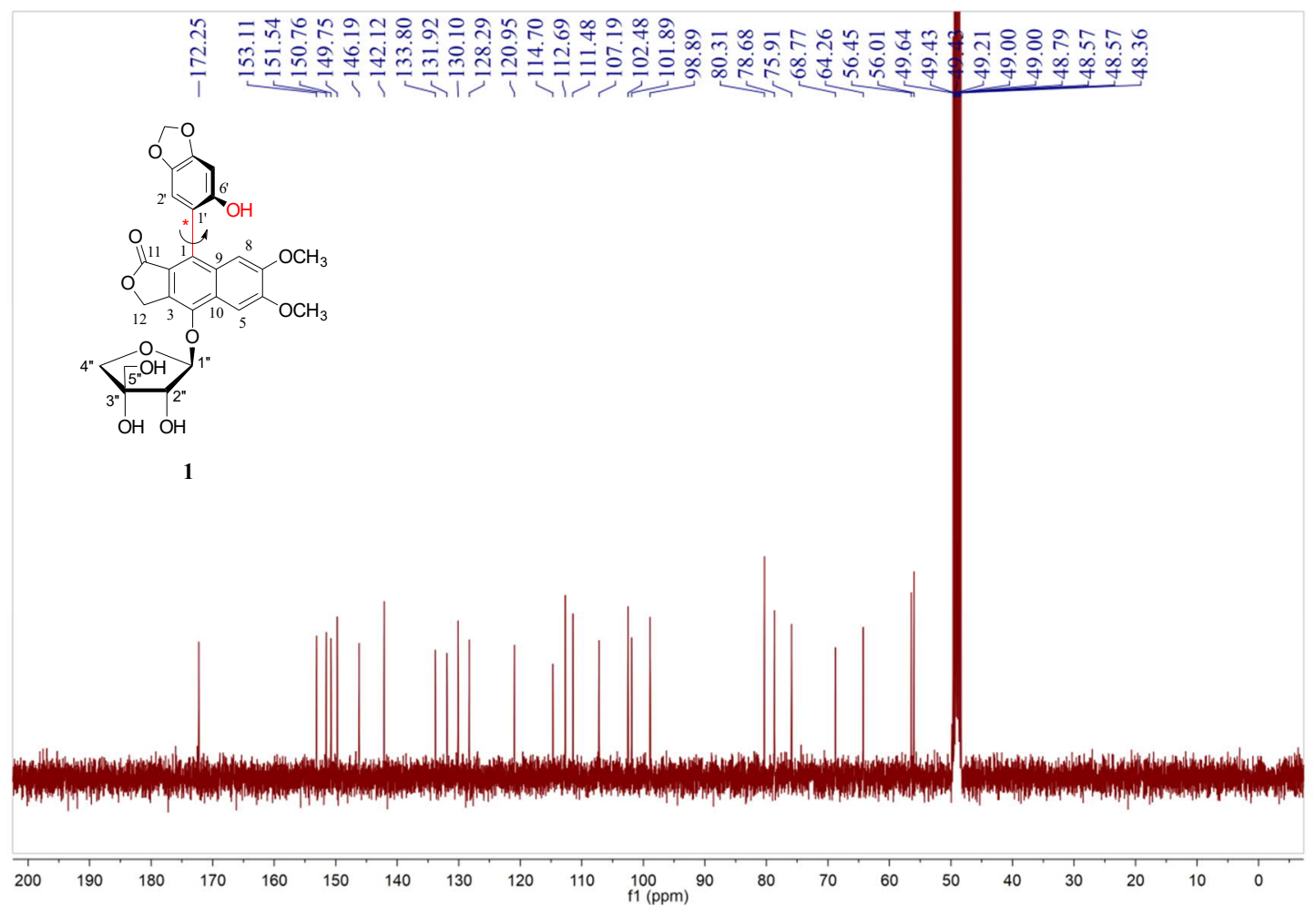

Figure S10. The ${ }^{13} \mathrm{C}\left\{{ }^{1} \mathrm{H}\right\}$ NMR Spectrum of Compound 1 in $\mathrm{CD}_{3} \mathrm{OD}(100 \mathrm{MHz})$. 


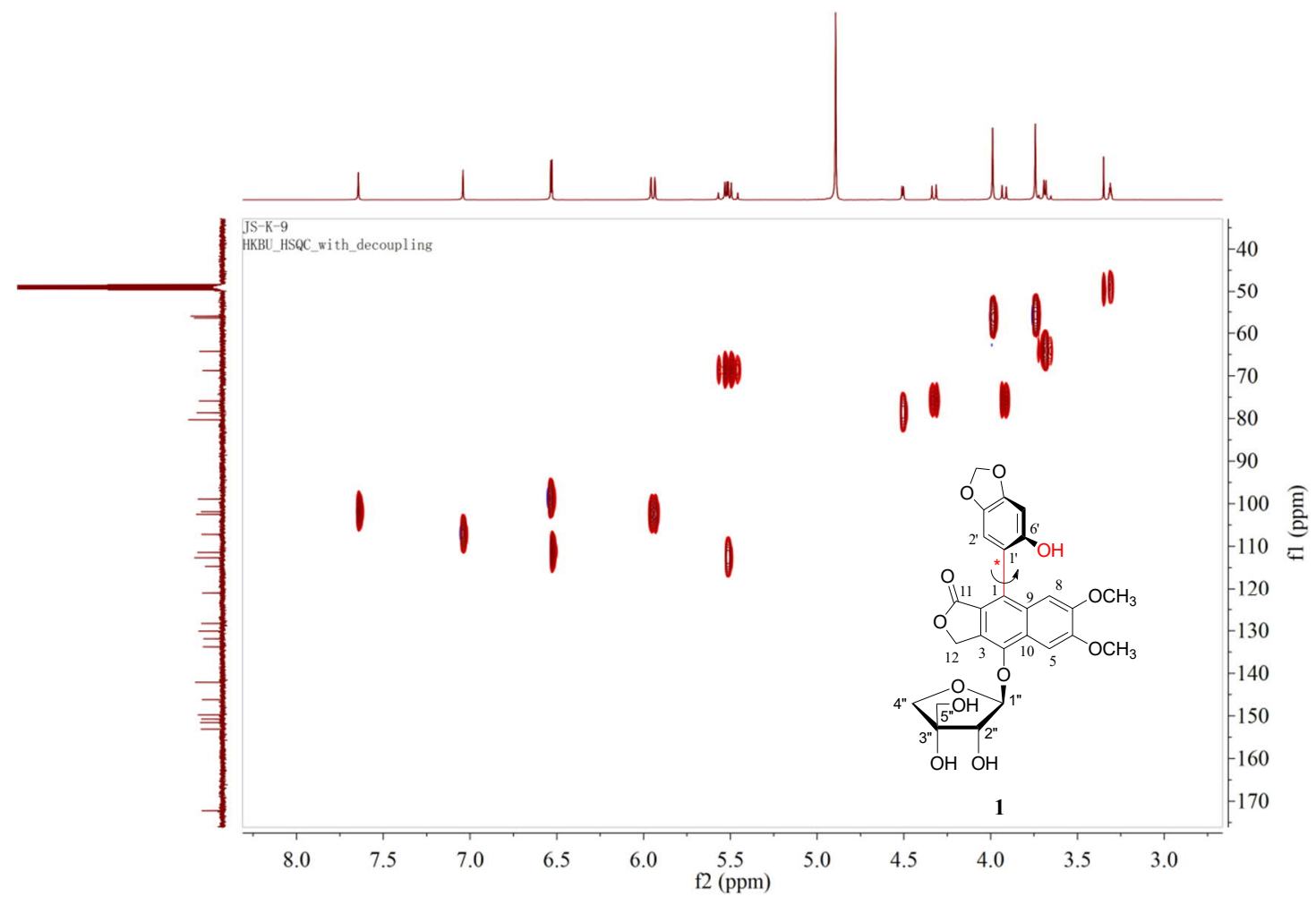

Figure S11. The HSQC Spectrum of Compound 1 in $\mathrm{CD}_{3} \mathrm{OD}\left(400 \mathrm{MHz}\right.$ for $\left.{ }^{1} \mathrm{H}\right)$.

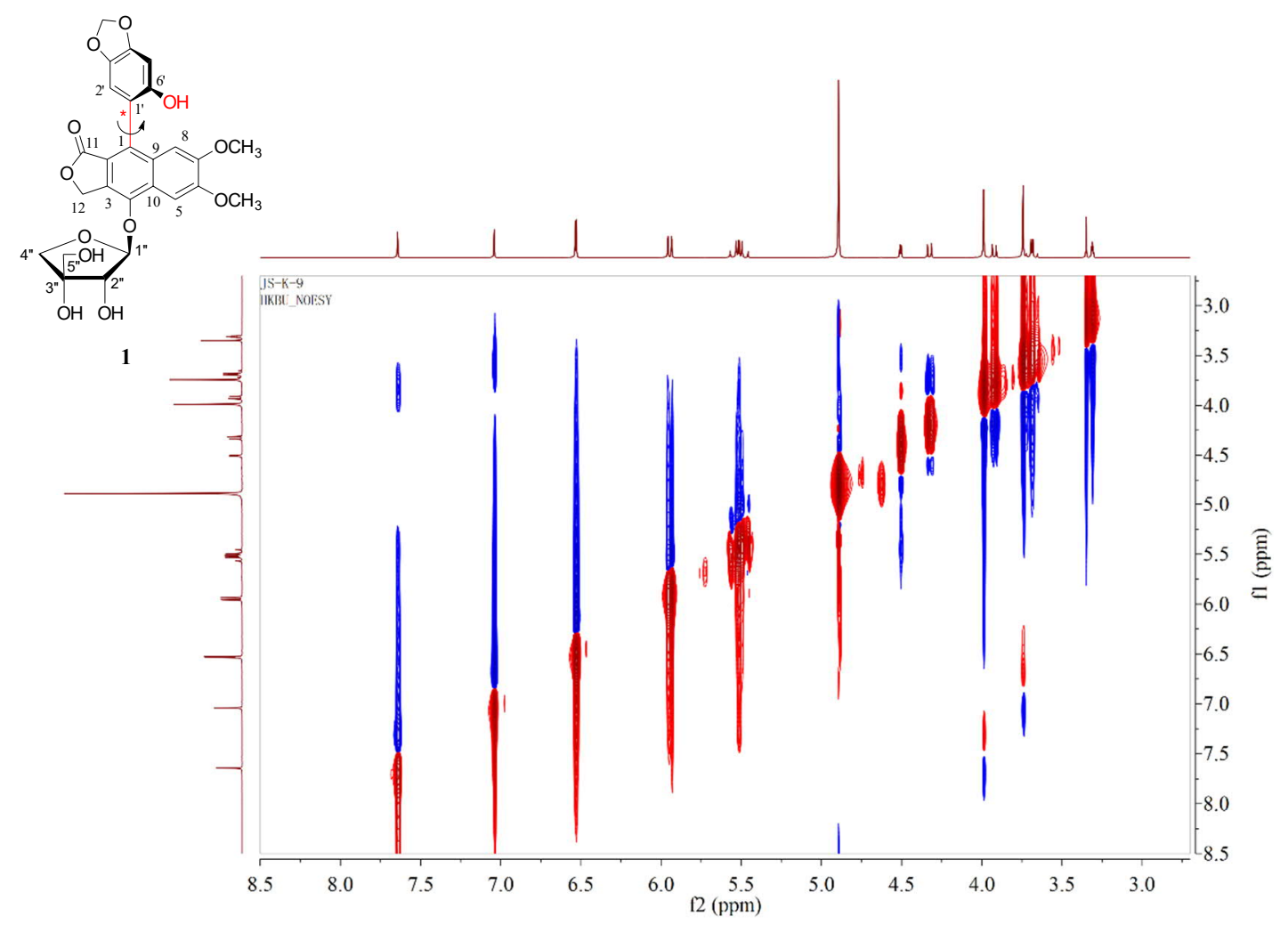

Figure S12. The NOESY Spectrum of Compound 1 in $\mathrm{CD}_{3} \mathrm{OD}(400 \mathrm{MHz})$. 


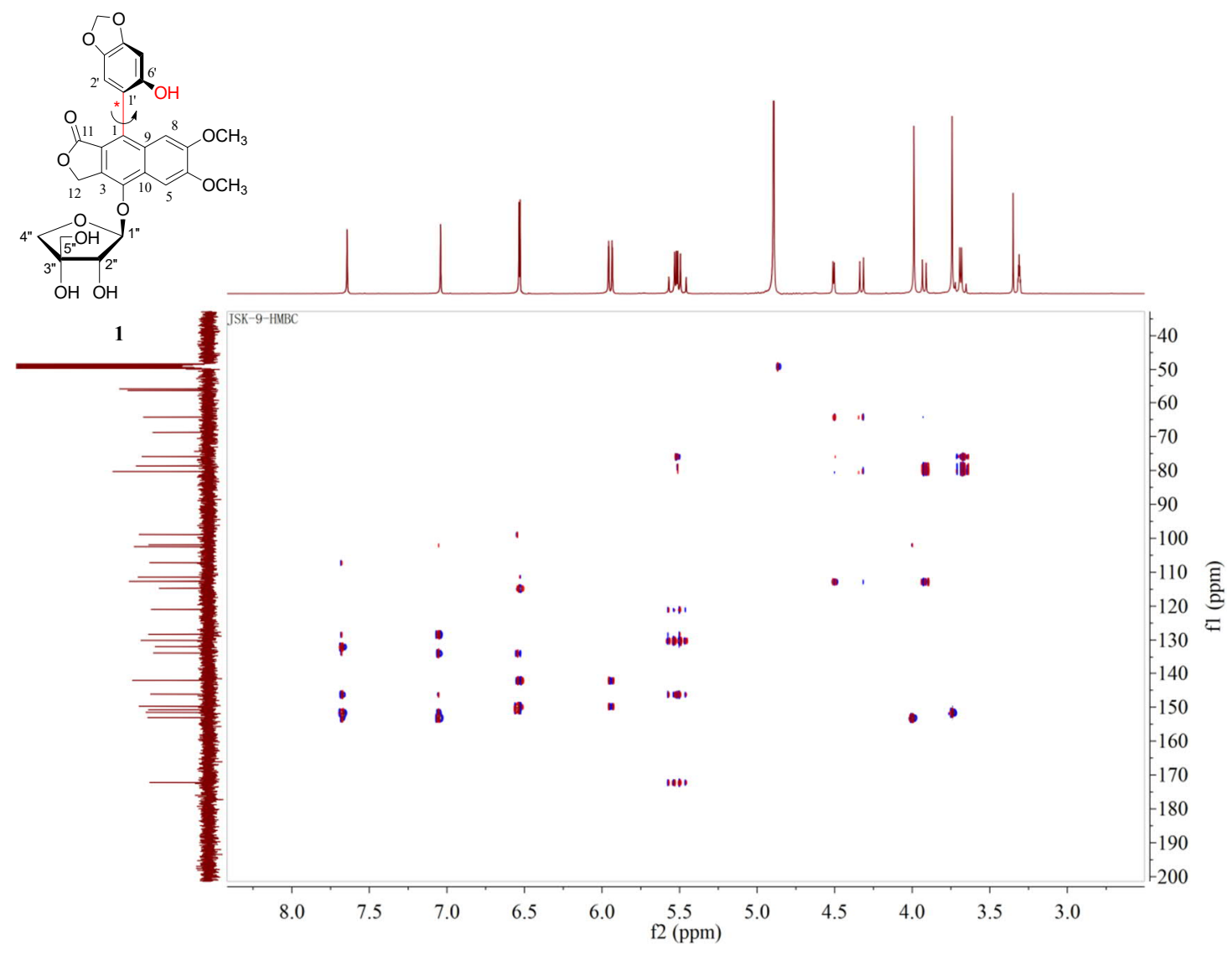

Figure S13. The HMBC Spectrum of Compound 1 in $\mathrm{CD}_{3} \mathrm{OD}\left(400 \mathrm{MHz}\right.$ for $\left.{ }^{1} \mathrm{H}\right)$.

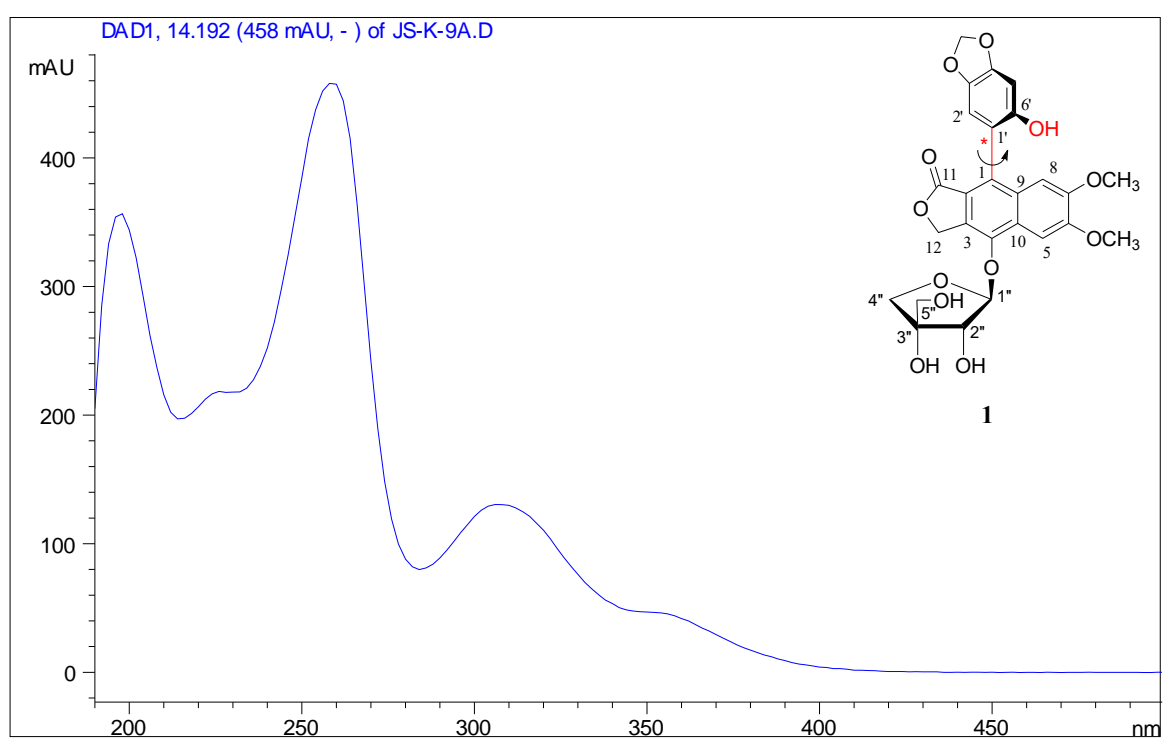

Figure S14. The UV Spectrum of Compound 1. 


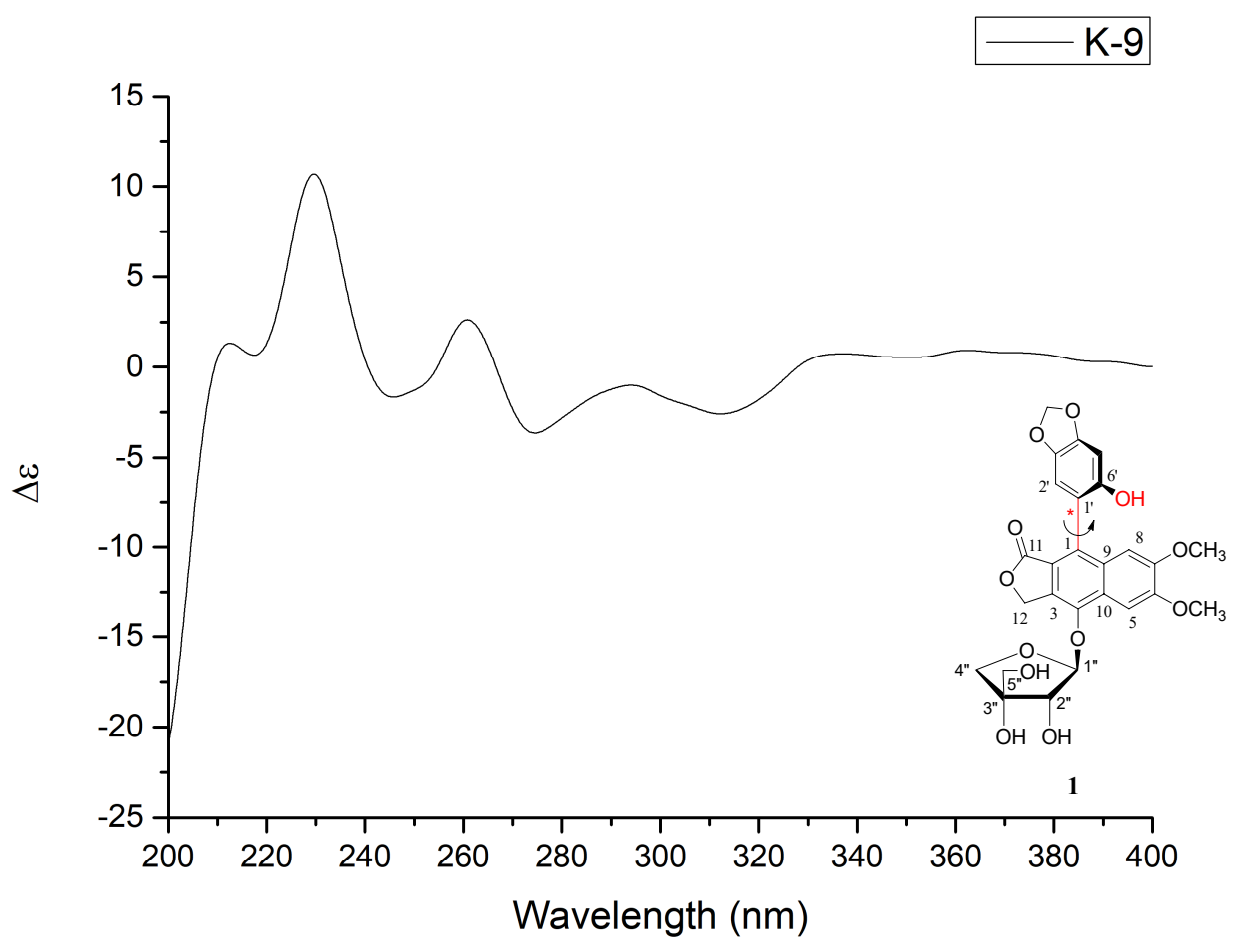

Figure S15. The CD Spectrum of Compound 1.

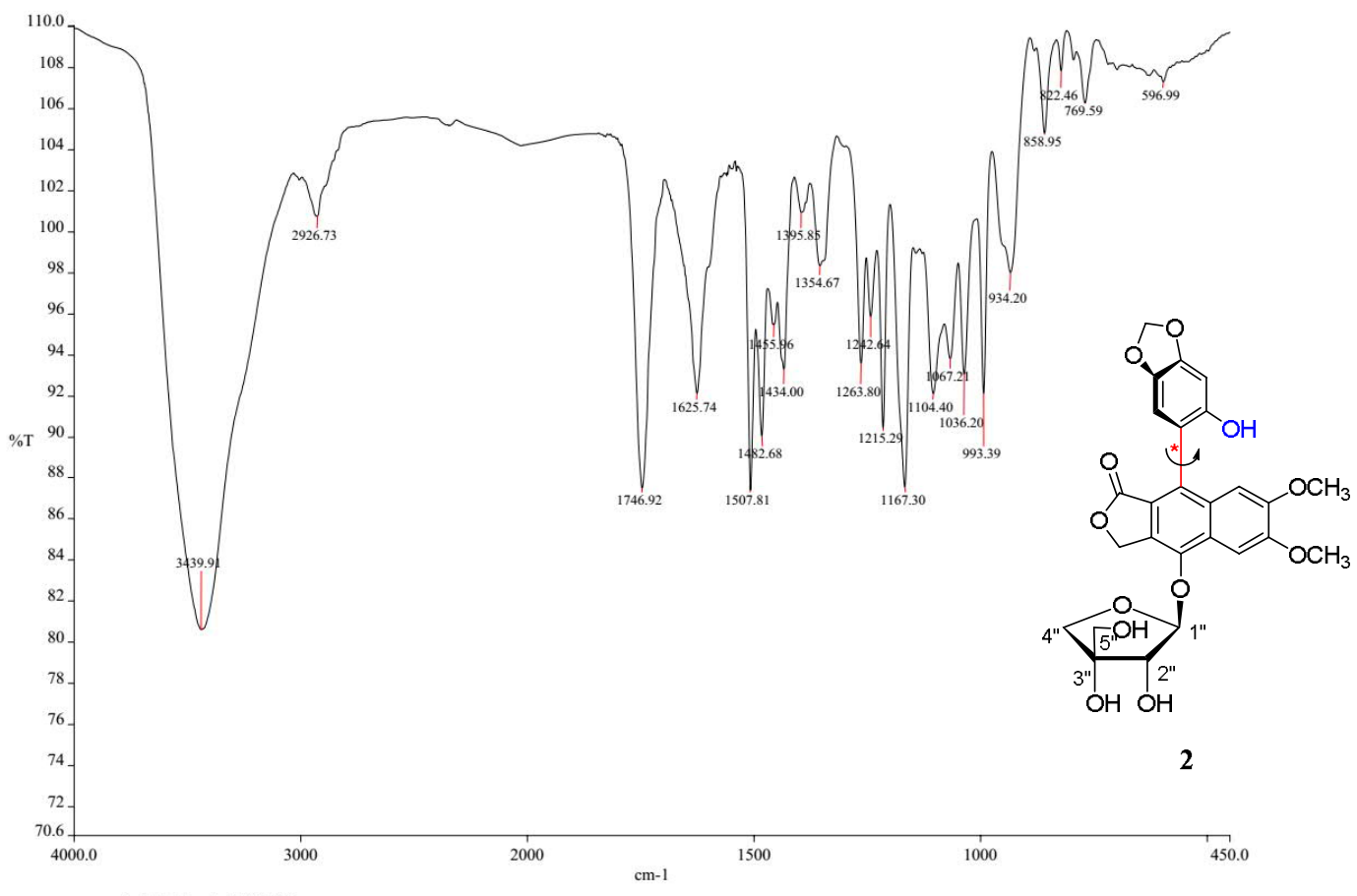

Figure S16. The IR Spectrum of Compound 2. 


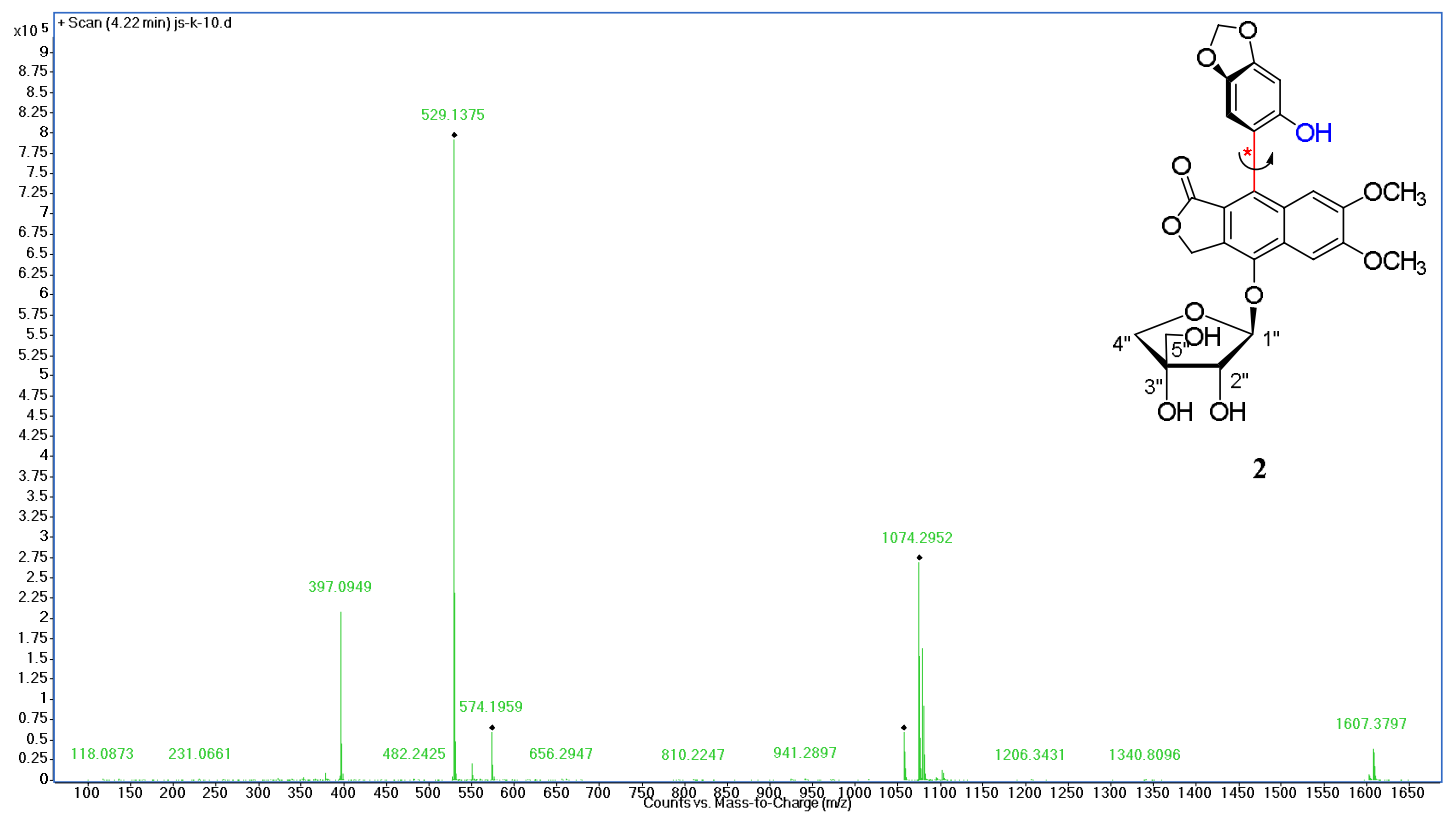

Figure S17. HRESIMS Data of Compound 2.

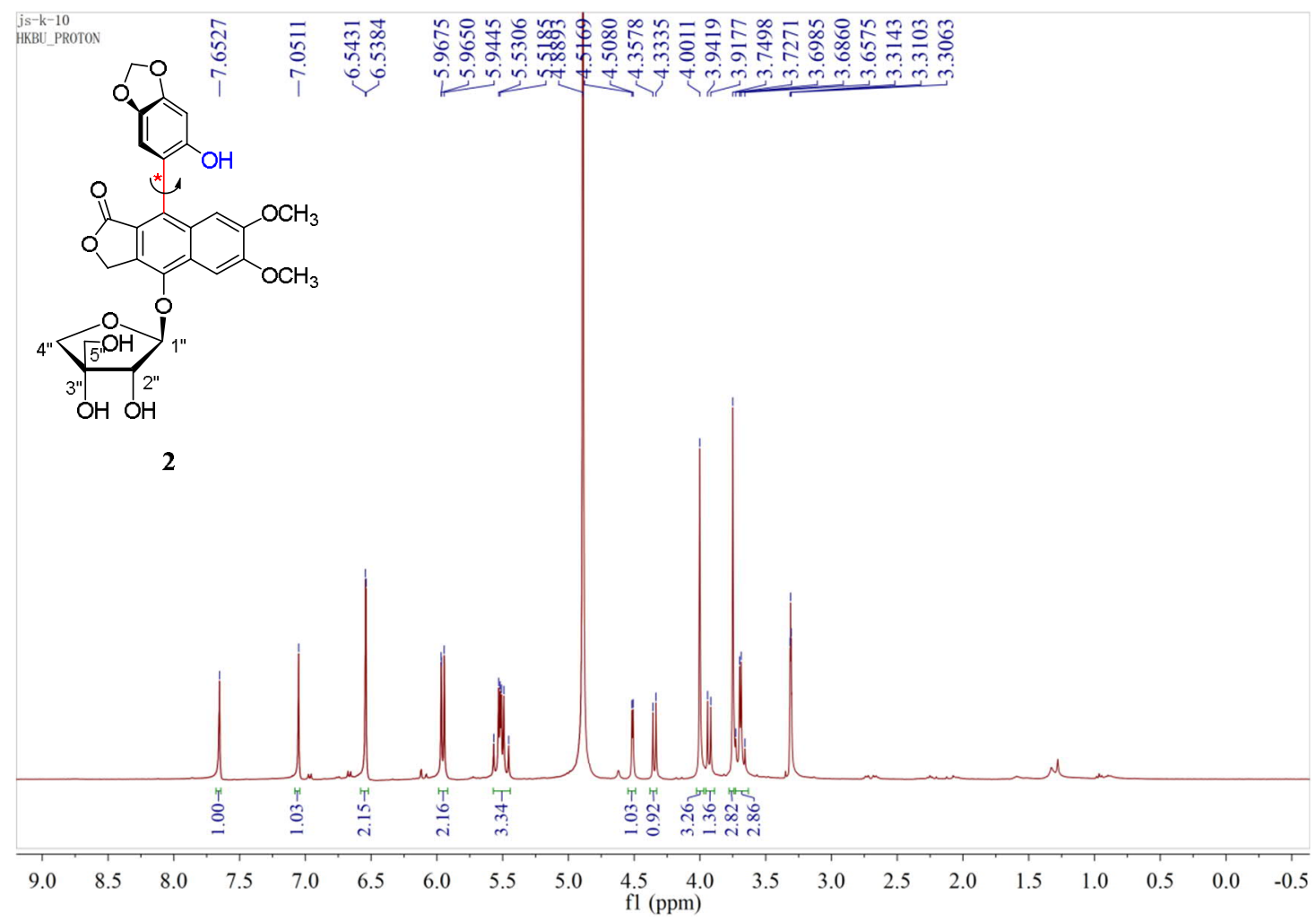

Figure S18. The ${ }^{1} \mathrm{H}$ NMR Spectrum of Compound 2 in $\mathrm{CD}_{3} \mathrm{OD}(400 \mathrm{MHz})$. 


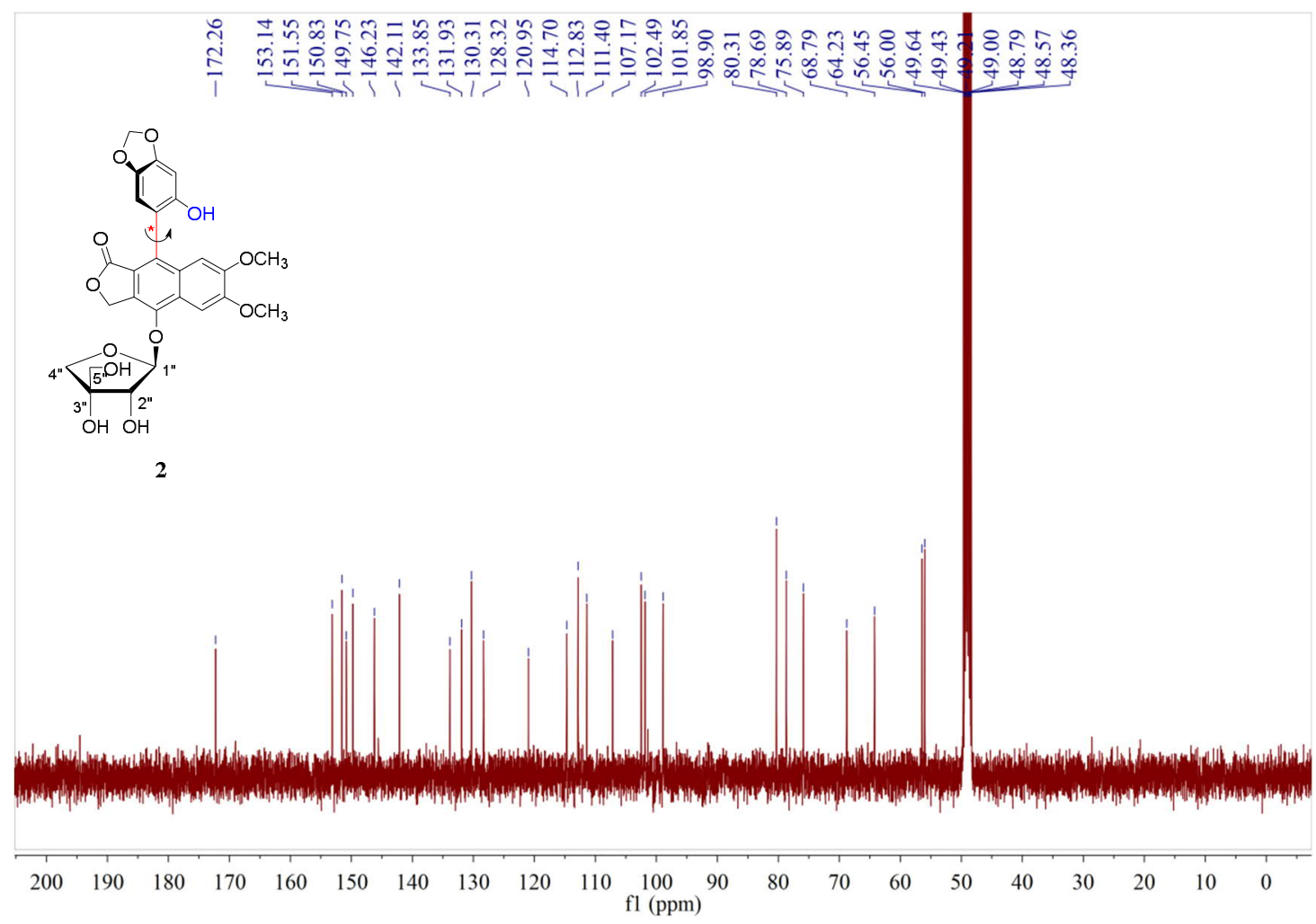

Figure S19. The ${ }^{13} \mathrm{C}\left\{{ }^{1} \mathrm{H}\right\}$ NMR Spectrum of Compound 2 in $\mathrm{CD}_{3} \mathrm{OD}(100 \mathrm{MHz})$.

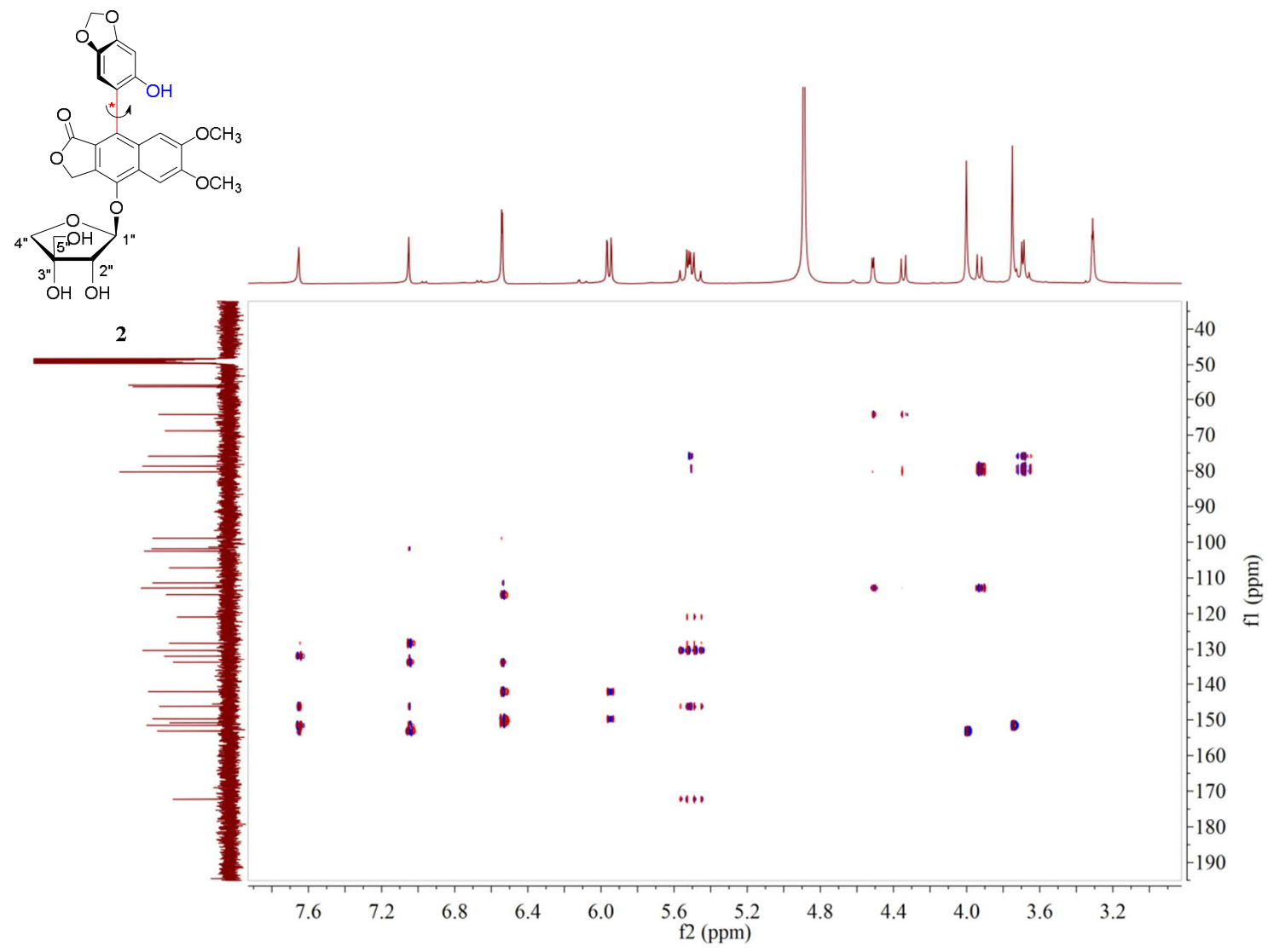

Figure S20. The HMBC Spectrum of Compound 2 in $\mathrm{CD}_{3} \mathrm{OD}\left(400 \mathrm{MHz}\right.$ for $\left.{ }^{1} \mathrm{H}\right)$. 


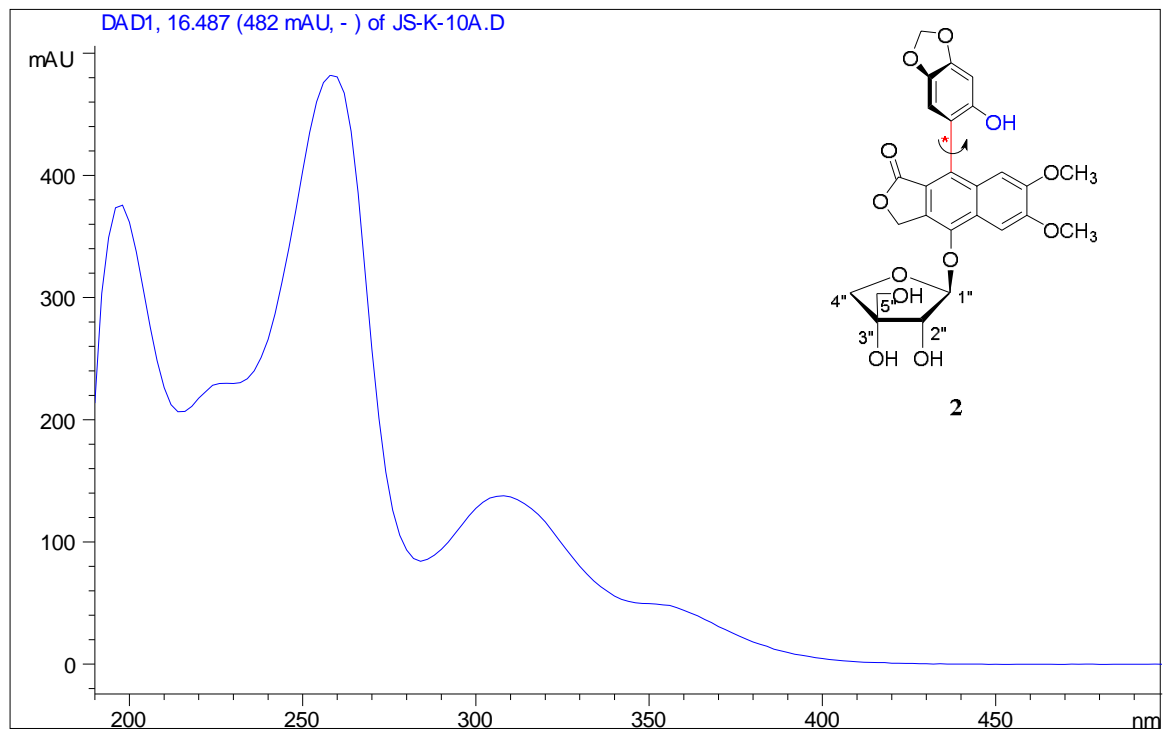

Figure S21. The UV Spectrum of Compound 2.

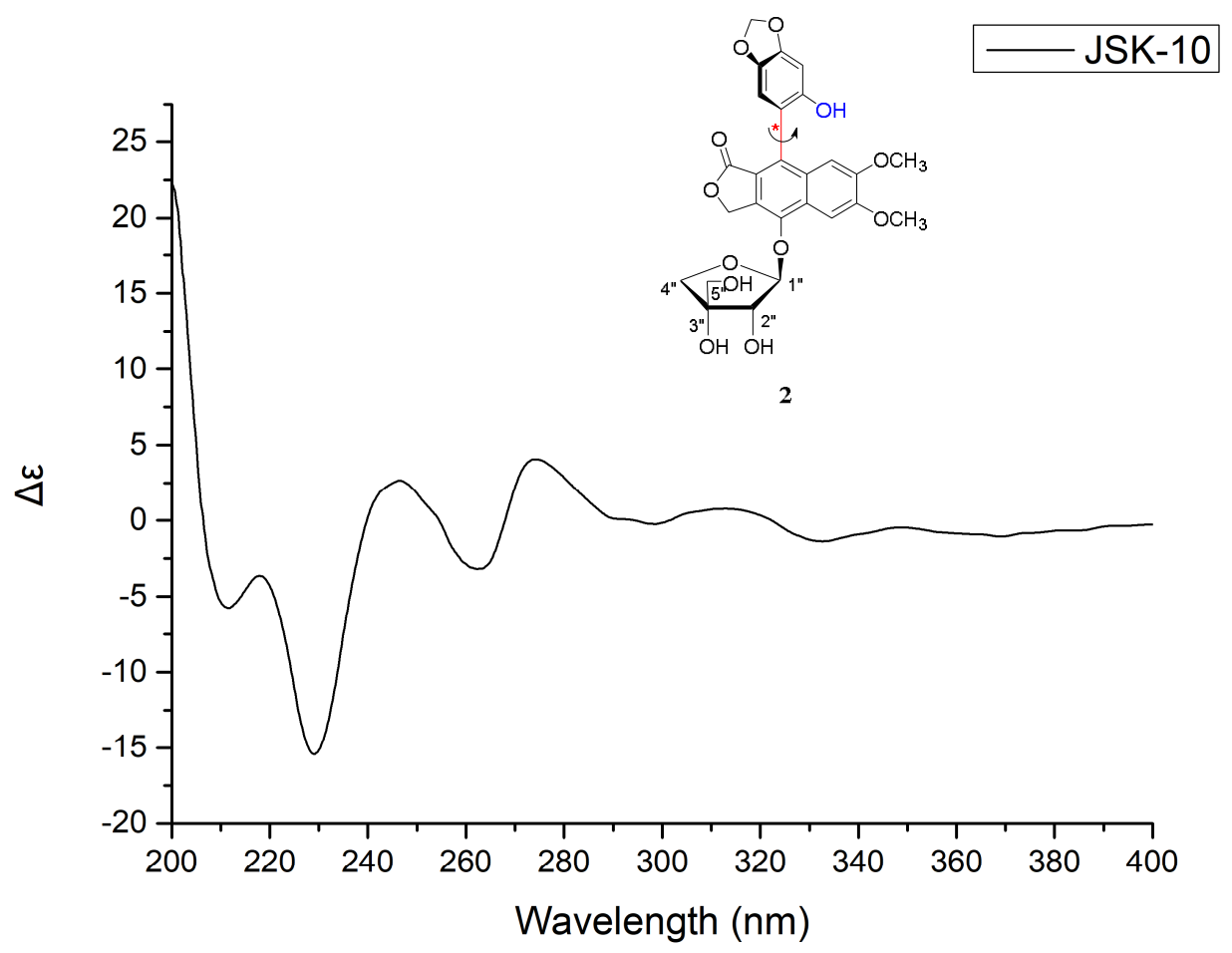

Figure S22. The CD Spectrum of Compound 2. 


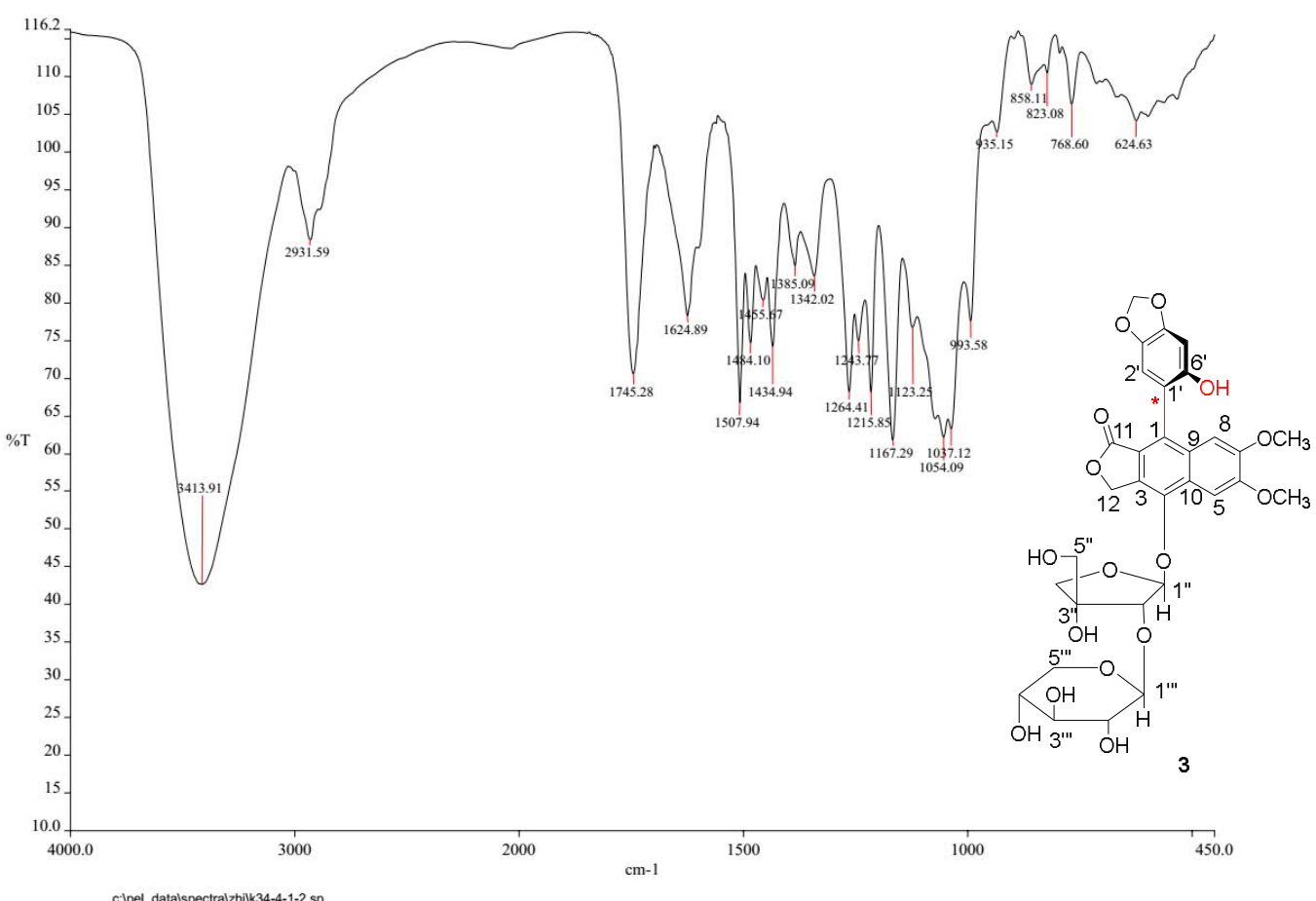

Figure S23. The IR Spectrum of Compound 3.

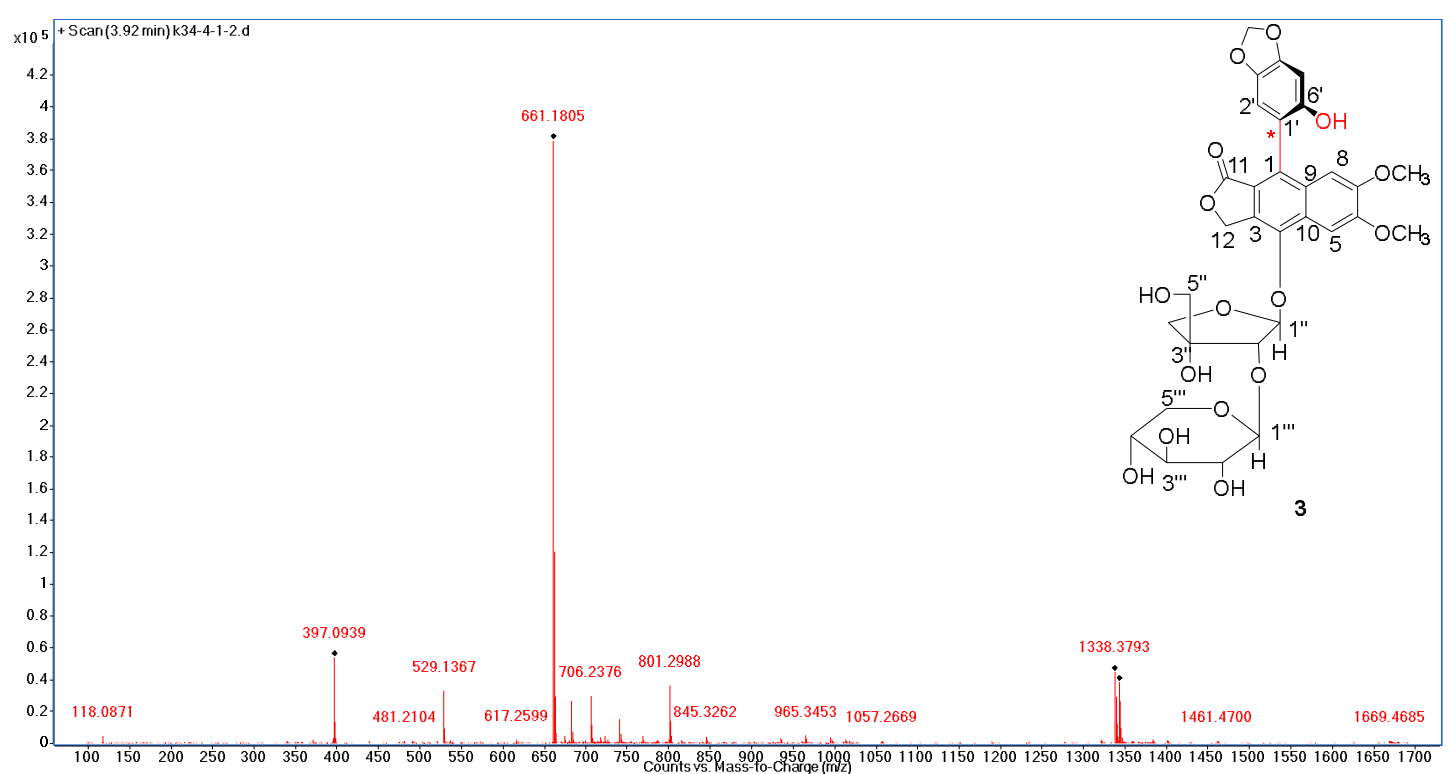

Figure S24. HRESIMS Data of Compound 3. 


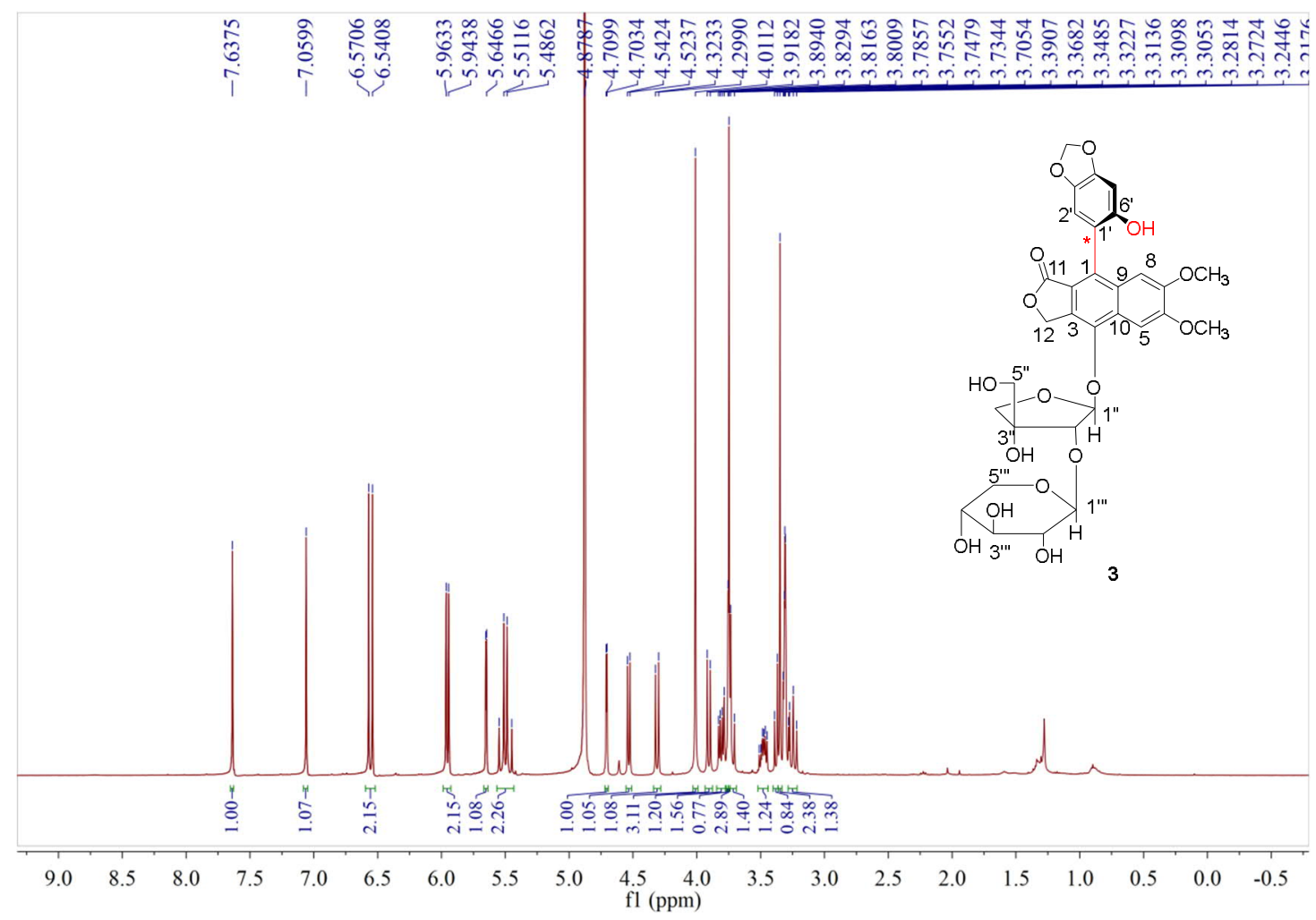

Figure S25. The ${ }^{1} \mathrm{H}$ NMR Spectrum of Compound 3 in $\mathrm{CD}_{3} \mathrm{OD}(400 \mathrm{MHz})$.

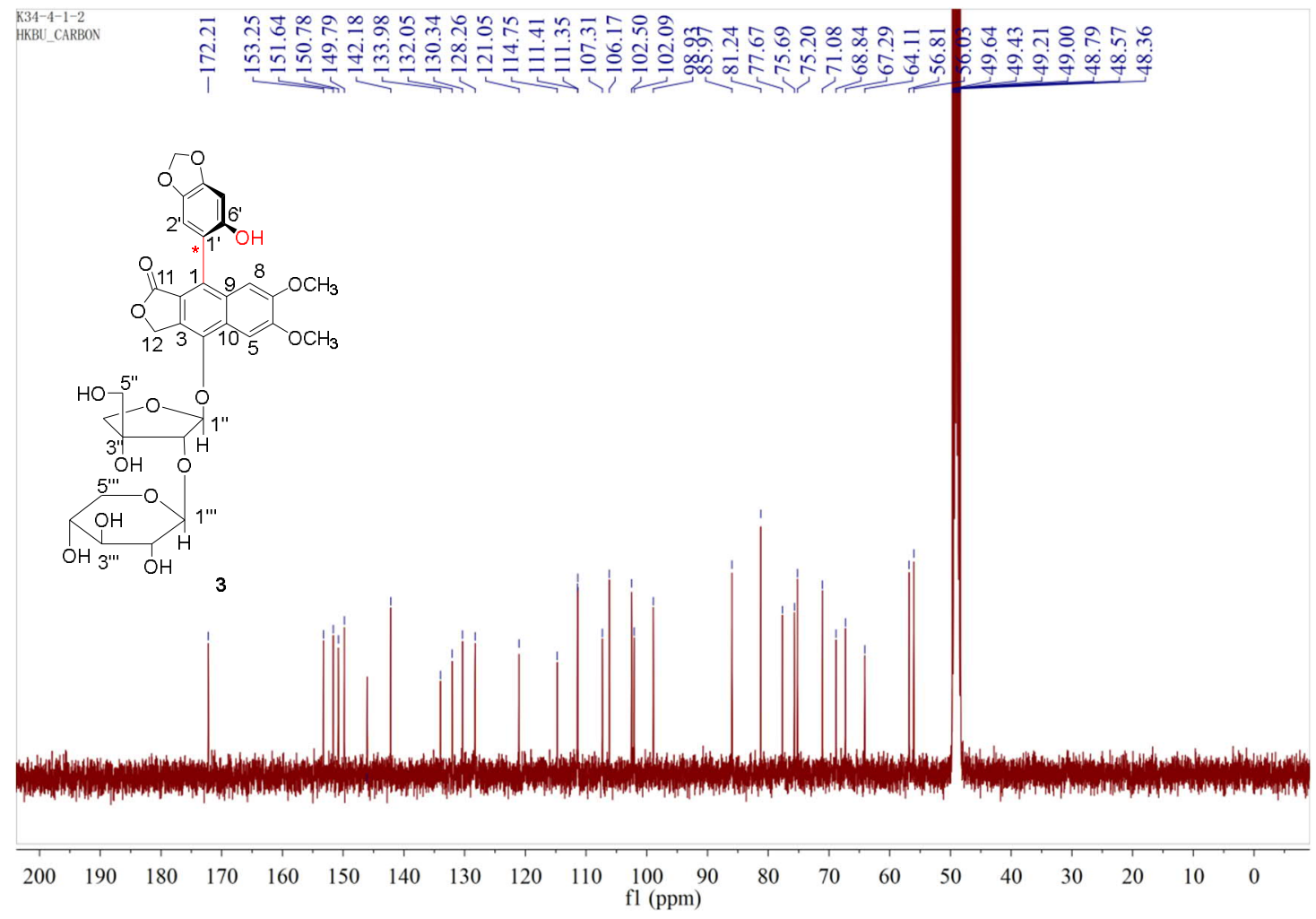

Figure S26. The ${ }^{13} \mathrm{C}\left\{{ }^{1} \mathrm{H}\right\}$ NMR Spectrum of Compound 3 in $\mathrm{CD}_{3} \mathrm{OD}(100 \mathrm{MHz})$. 


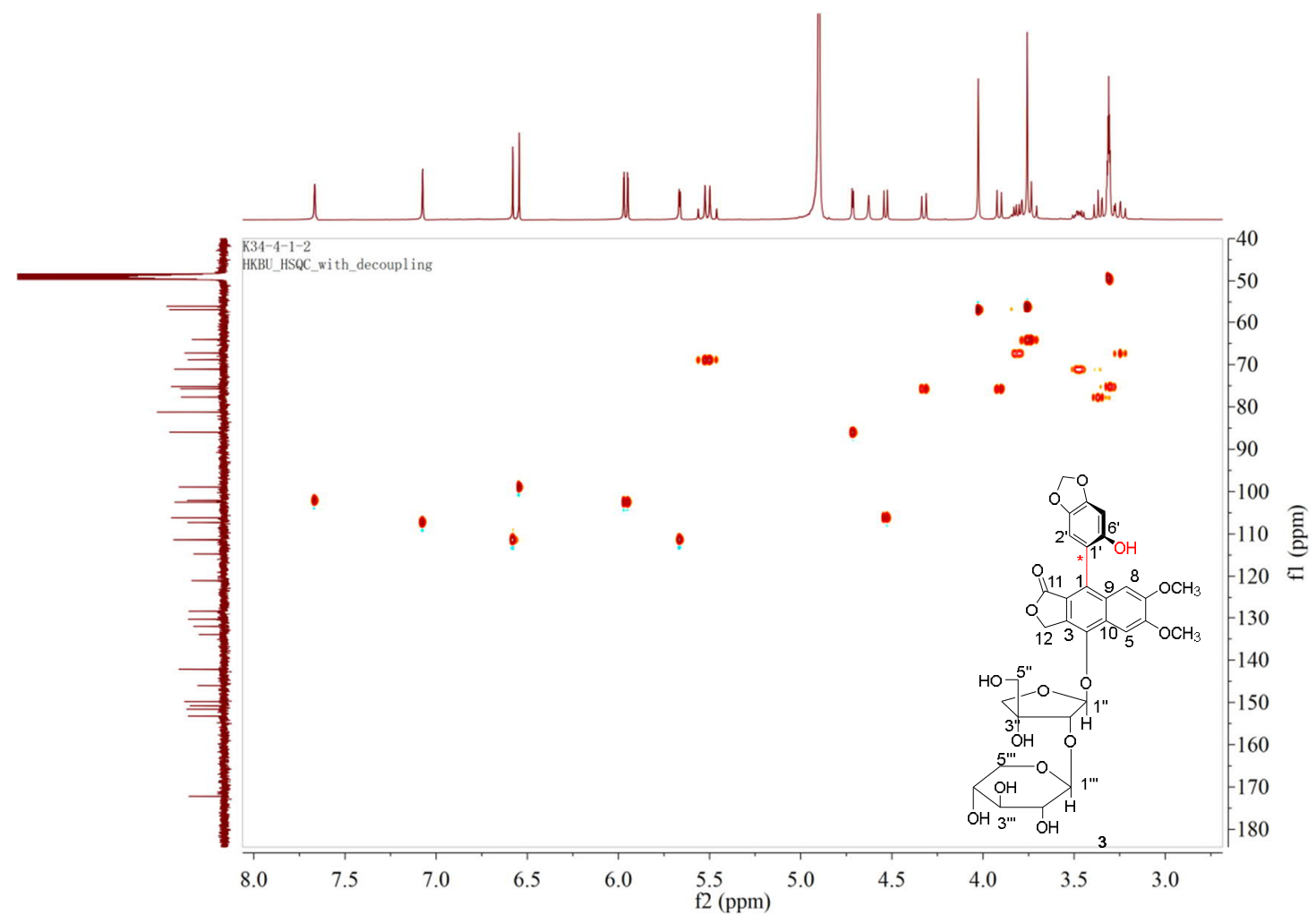

Figure S27. The HSQC Spectrum of Compound 3 in $\mathrm{CD}_{3} \mathrm{OD}\left(400 \mathrm{MHz}\right.$ for $\left.{ }^{1} \mathrm{H}\right)$.

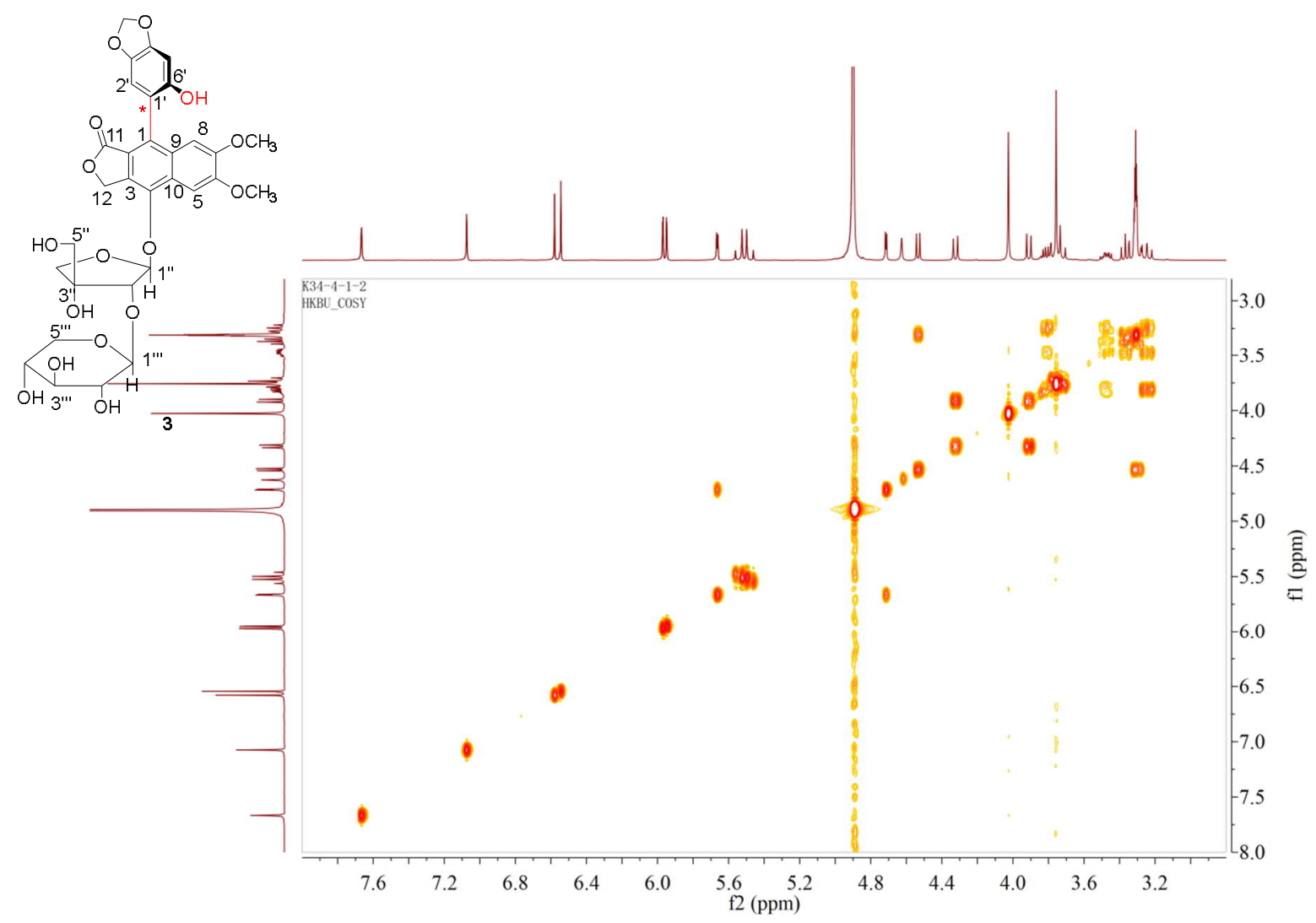

Figure S28. The ${ }^{1} \mathrm{H}-{ }^{1} \mathrm{H}$ COSY Spectrum of Compound 3 in $\mathrm{CD}_{3} \mathrm{OD}(400 \mathrm{MHz})$. 


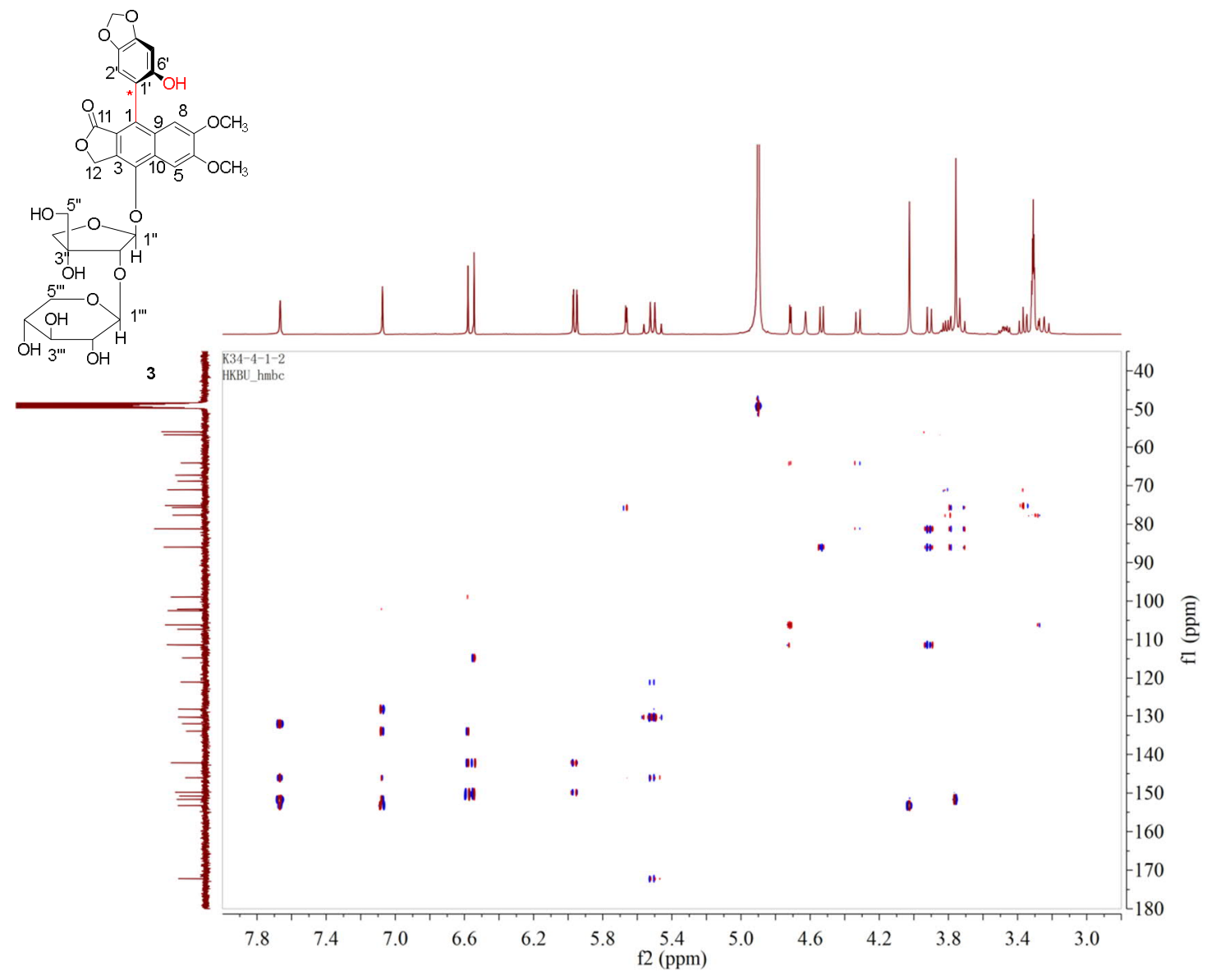

Figure S29. The HMBC Spectrum of Compound 3 in $\mathrm{CD}_{3} \mathrm{OD}\left(400 \mathrm{MHz}\right.$ for $\left.{ }^{1} \mathrm{H}\right)$.

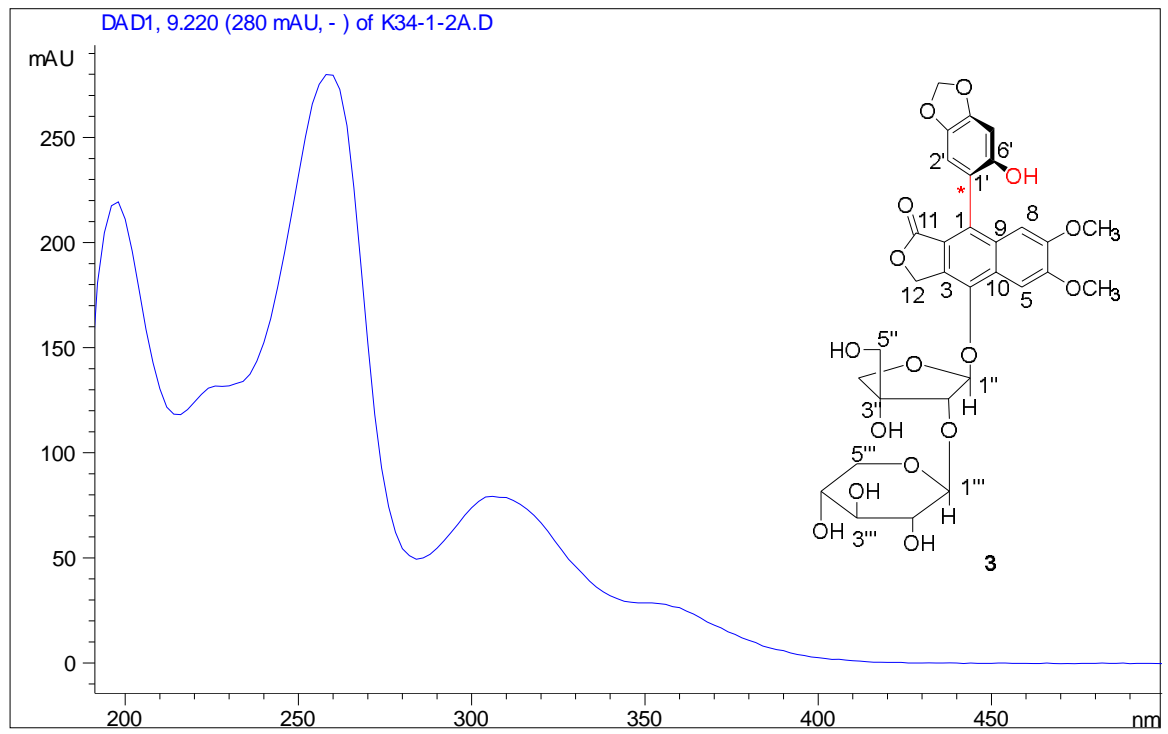

Figure S30. The UV Spectrum of Compound 3. 


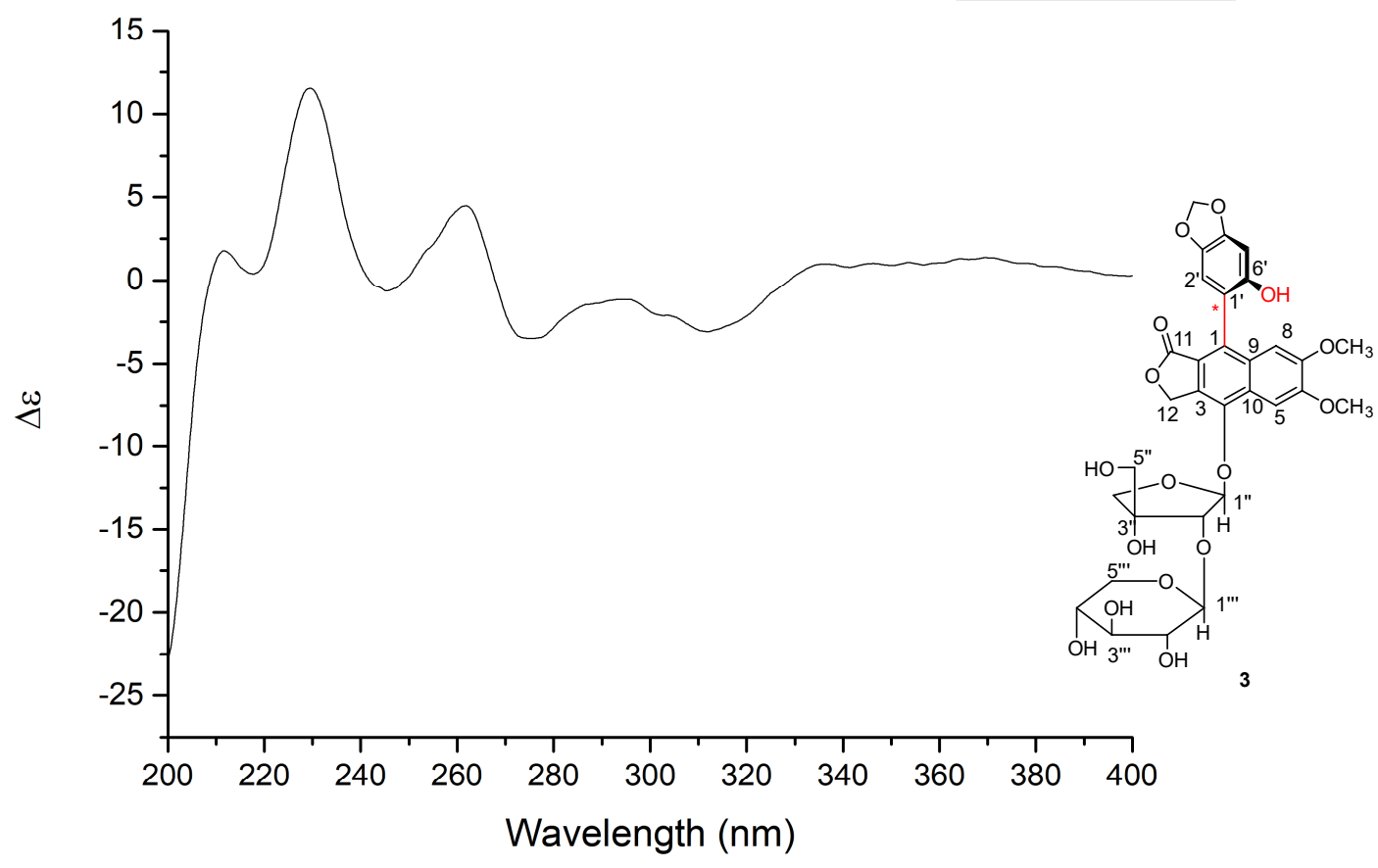

Figure S31. The CD Spectrum of Compound 3.

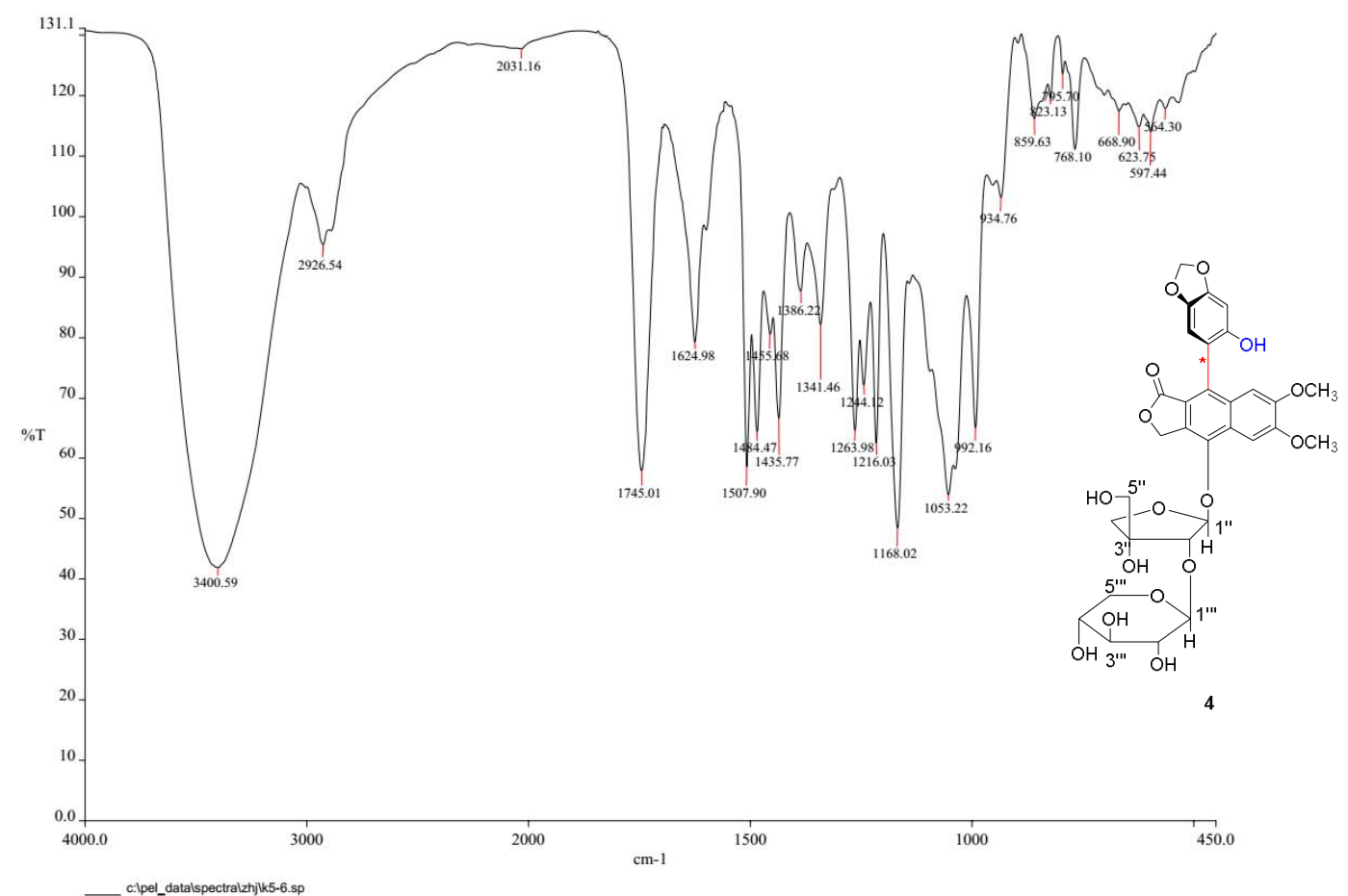

Figure S32. The IR Spectrum of Compound 4. 


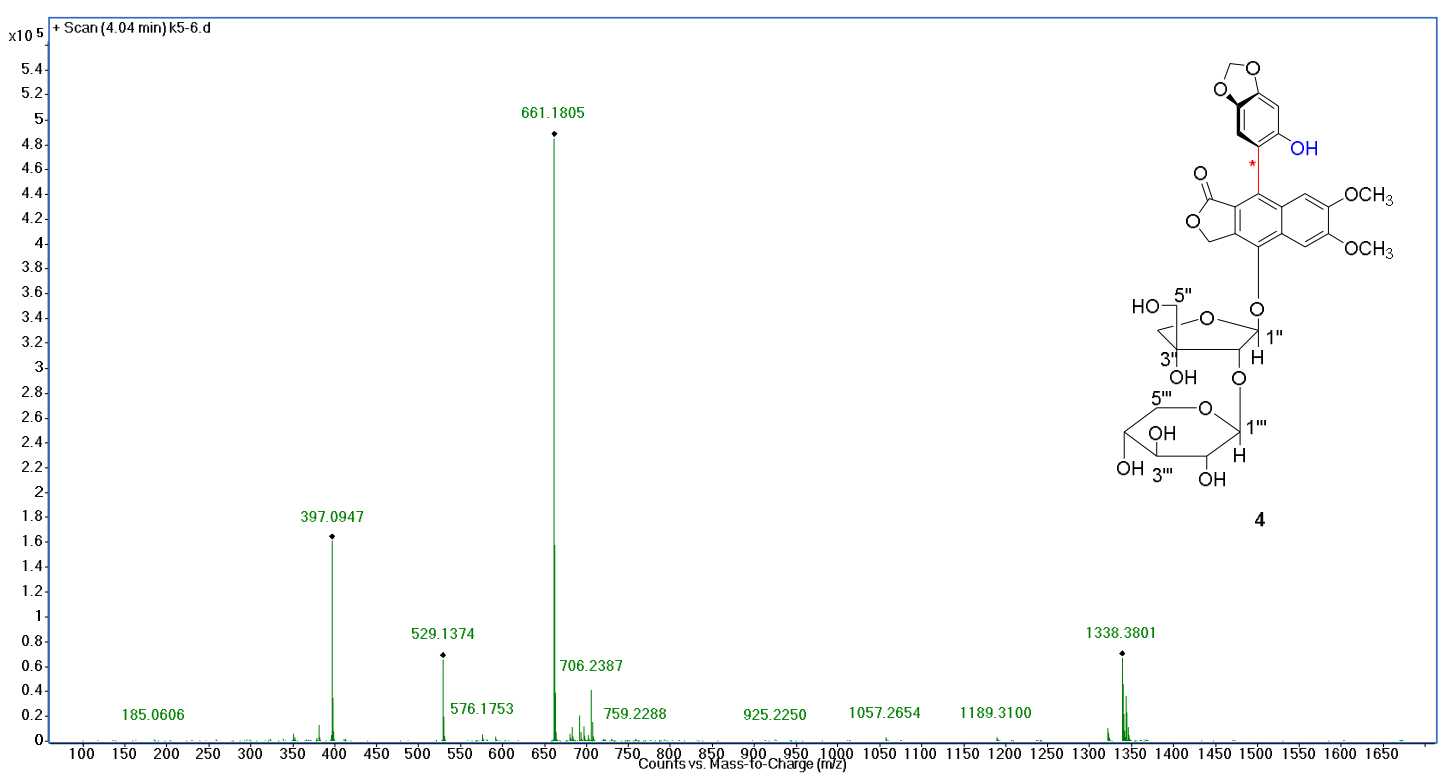

Figure S33. HRESIMS Data of Compound 4.

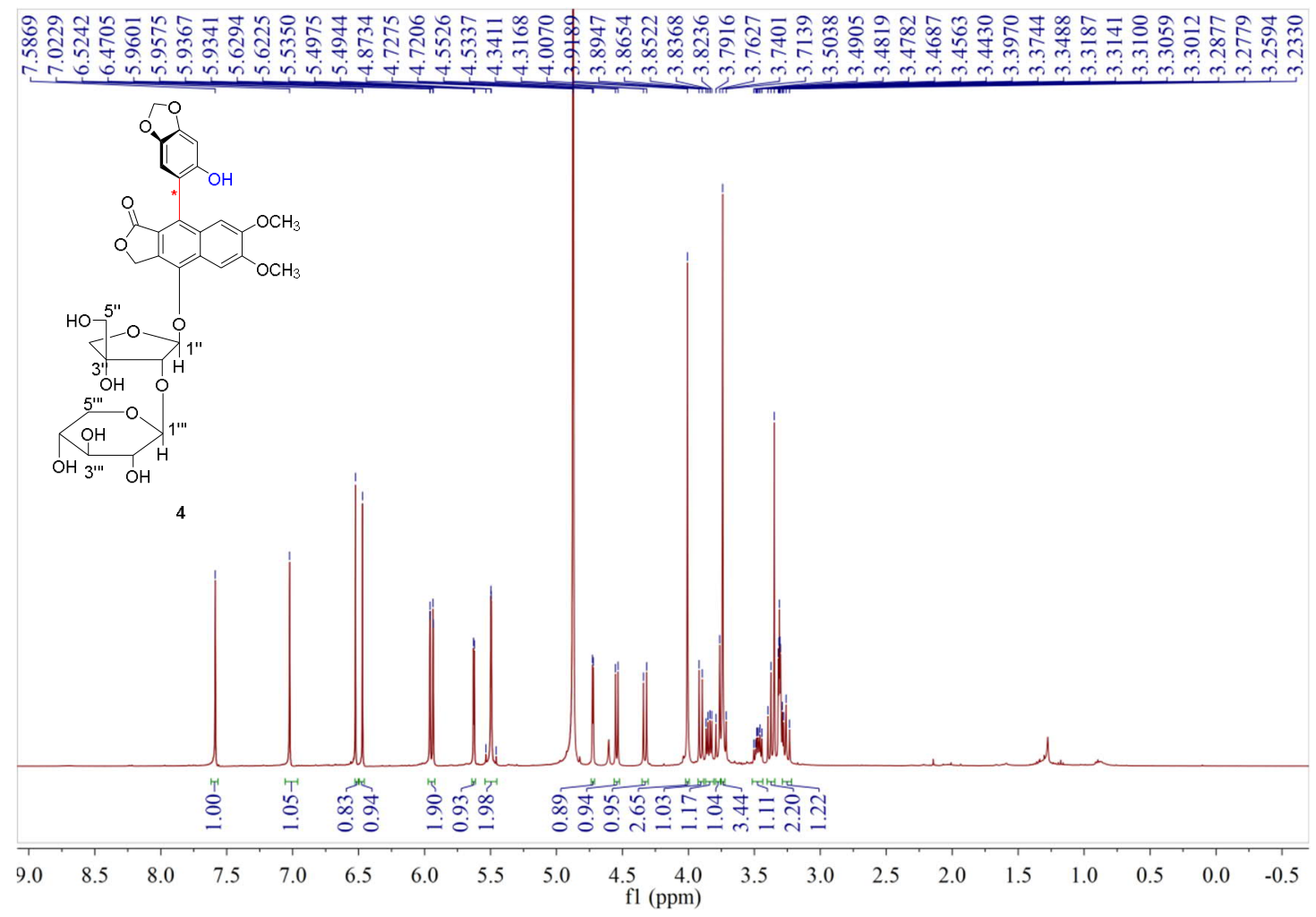

Figure S34. The ${ }^{1} \mathrm{H}$ NMR Spectrum of Compound 4 in $\mathrm{CD}_{3} \mathrm{OD}(400 \mathrm{MHz})$. 


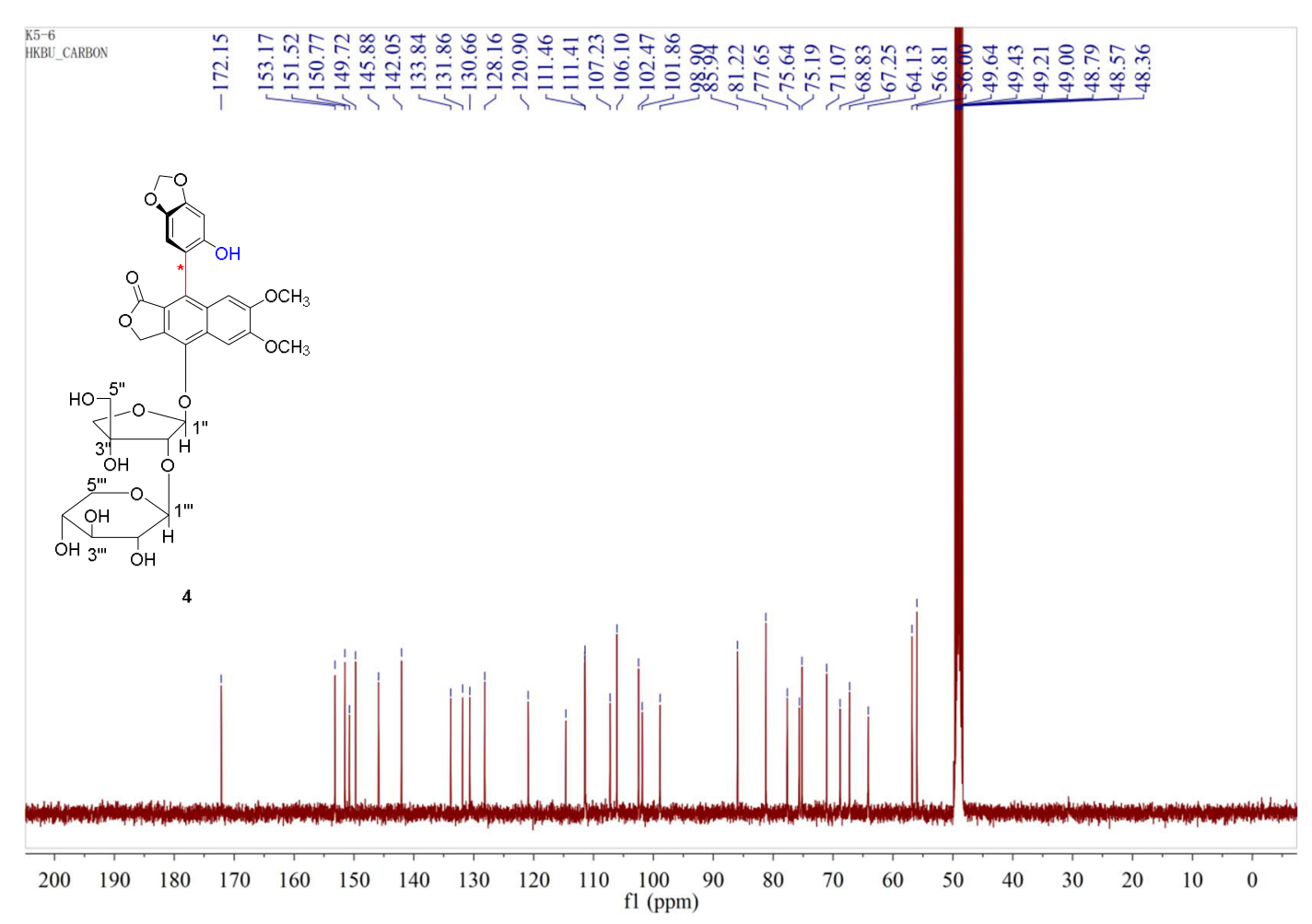

Figure S35. The ${ }^{13} \mathrm{C}\left\{{ }^{1} \mathrm{H}\right\}$ NMR Spectrum of Compound 4 in $\mathrm{CD}_{3} \mathrm{OD}(100 \mathrm{MHz})$.

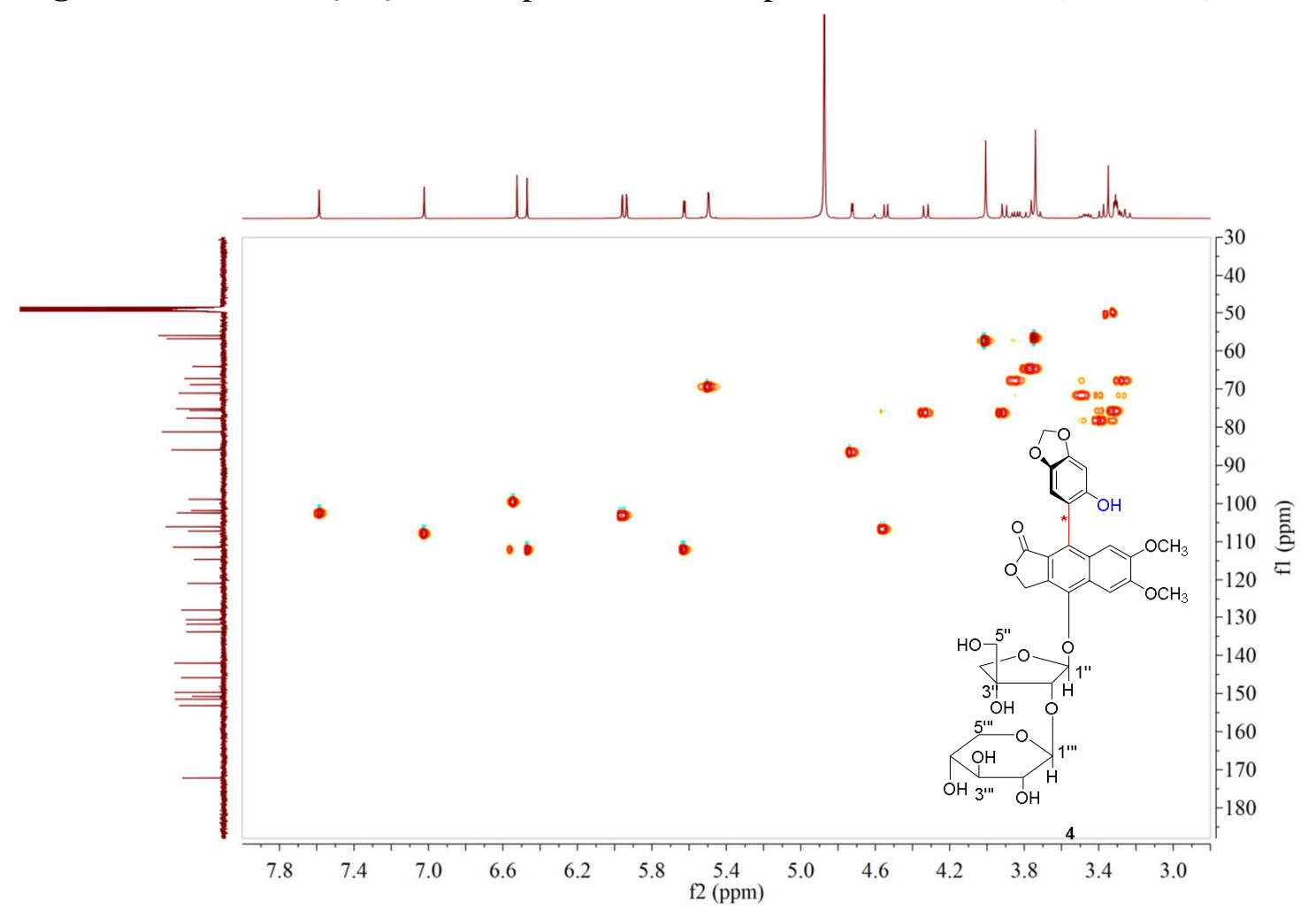

Figure S36. The HSQC Spectrum of Compound 4 in $\mathrm{CD}_{3} \mathrm{OD}\left(400 \mathrm{MHz}\right.$ for $\left.{ }^{1} \mathrm{H}\right)$. 


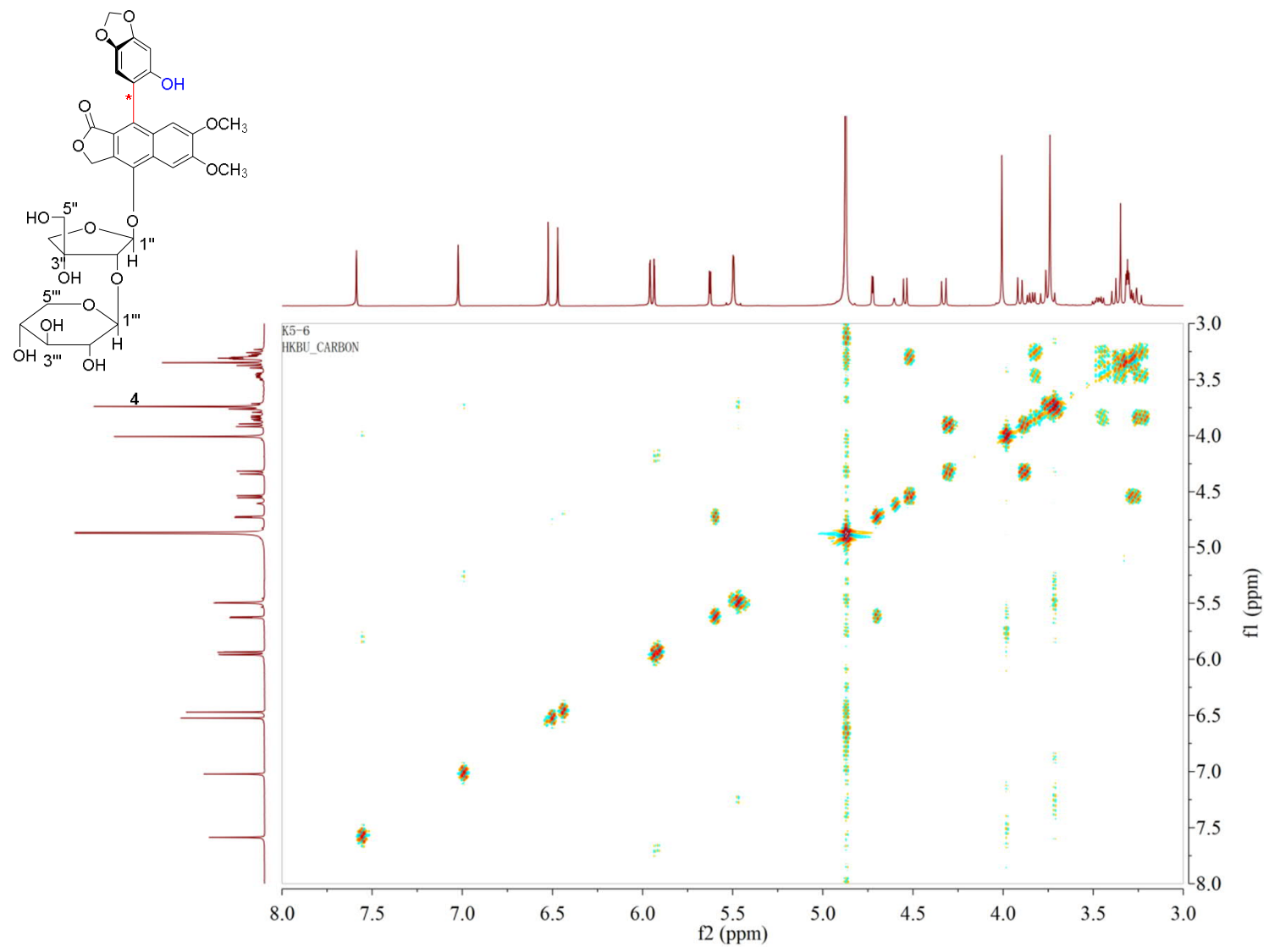

Figure S37. The ${ }^{1} \mathrm{H}-{ }^{1} \mathrm{H}$ COSY Spectrum of Compound 4 in $\mathrm{CD}_{3} \mathrm{OD}(400 \mathrm{MHz})$.

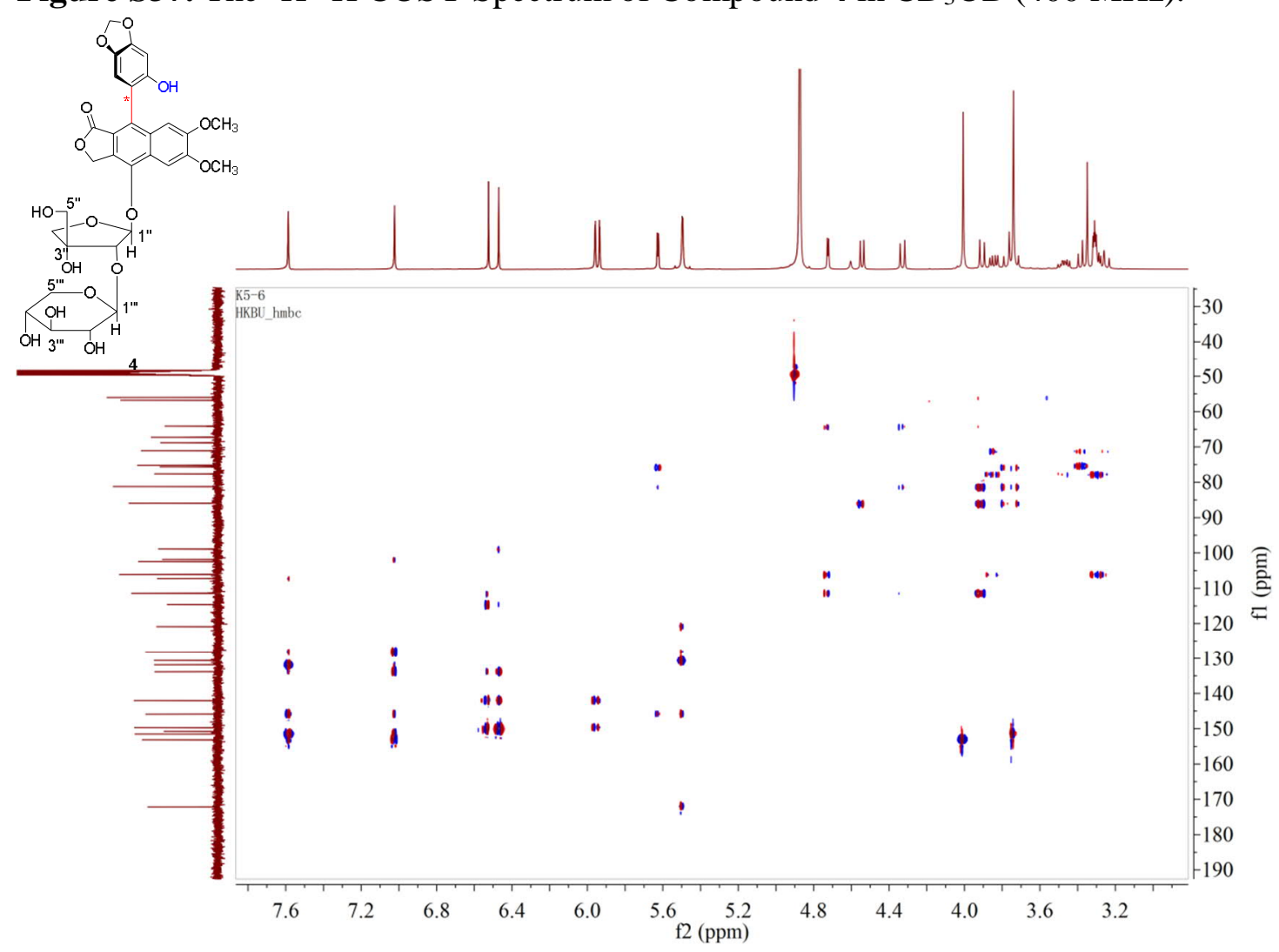

Figure S38. The HMBC Spectrum of Compound 4 in $\mathrm{CD}_{3} \mathrm{OD}\left(400 \mathrm{MHz}\right.$ for $\left.{ }^{1} \mathrm{H}\right)$. 


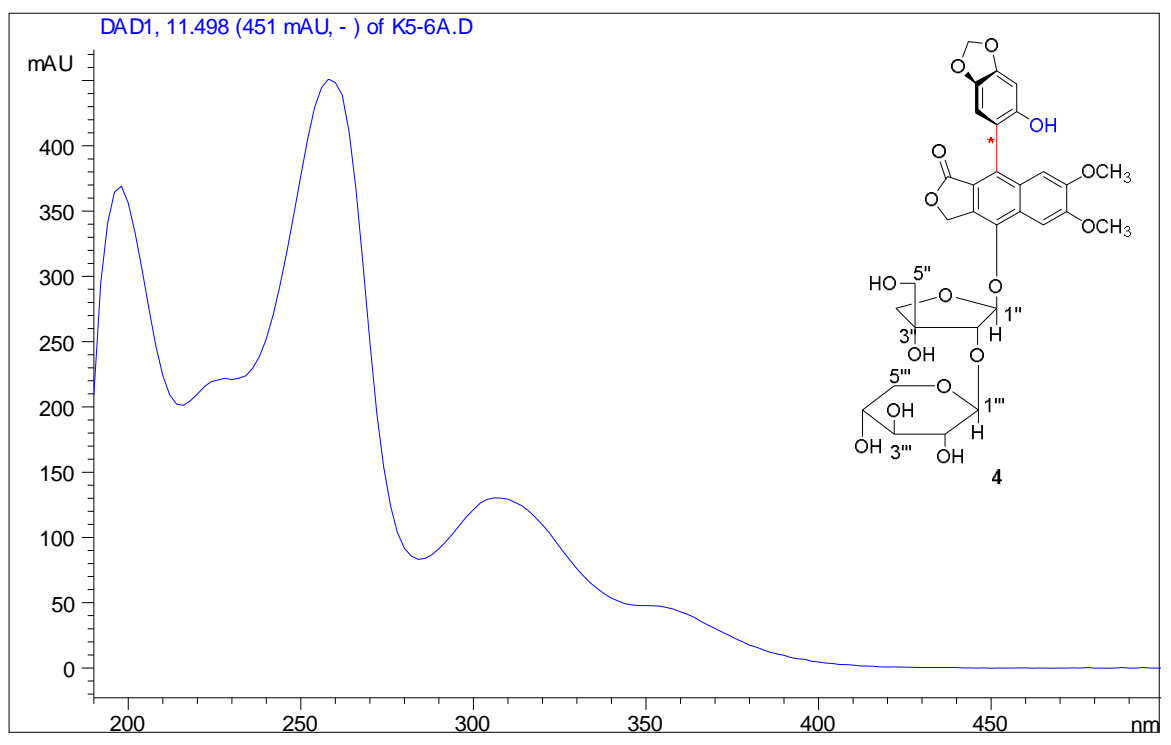

Figure S39. The UV Spectrum of Compound 4.

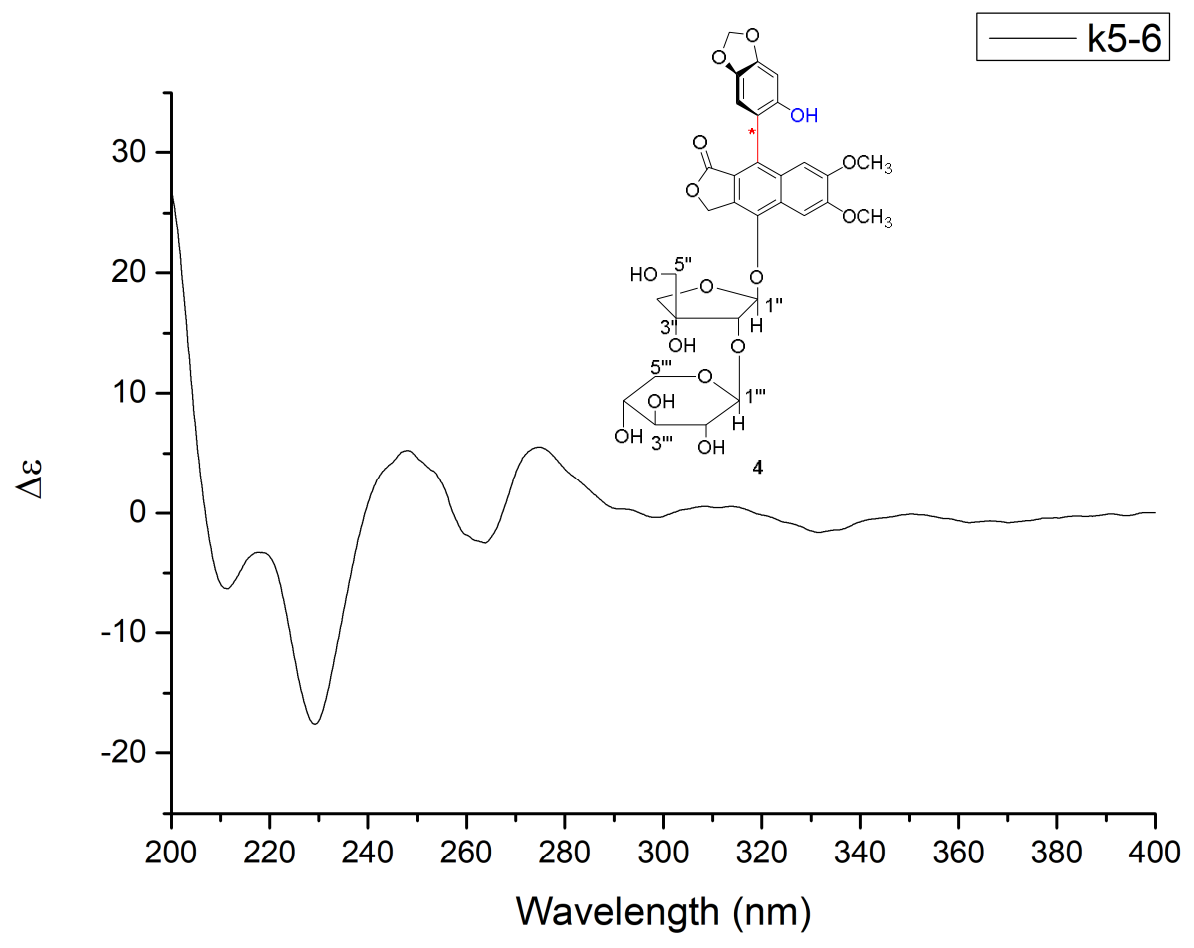

Figure S40. The CD Spectrum of Compound 4. 


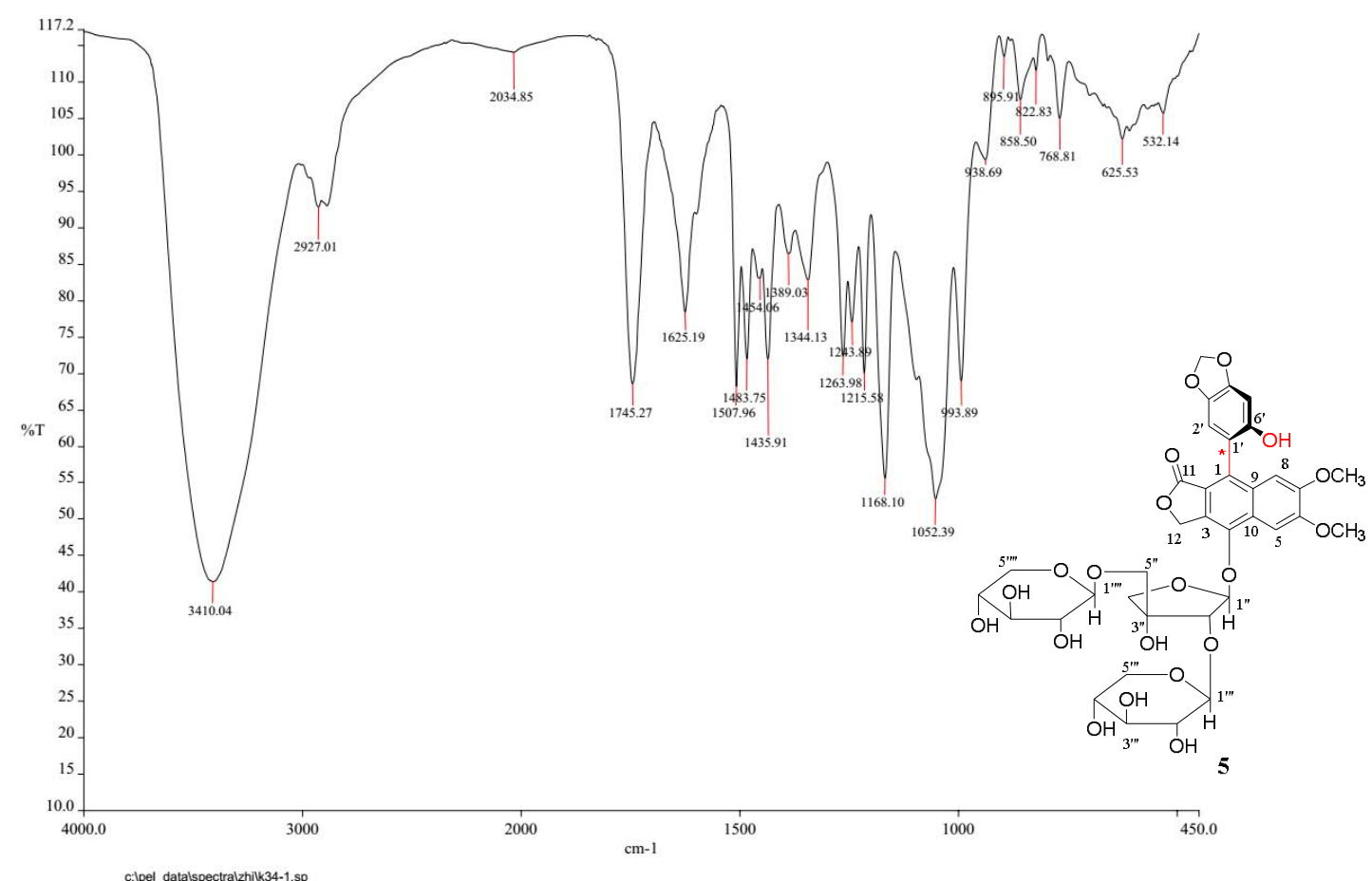

Figure S41. The IR Spectrum of Compound 5.

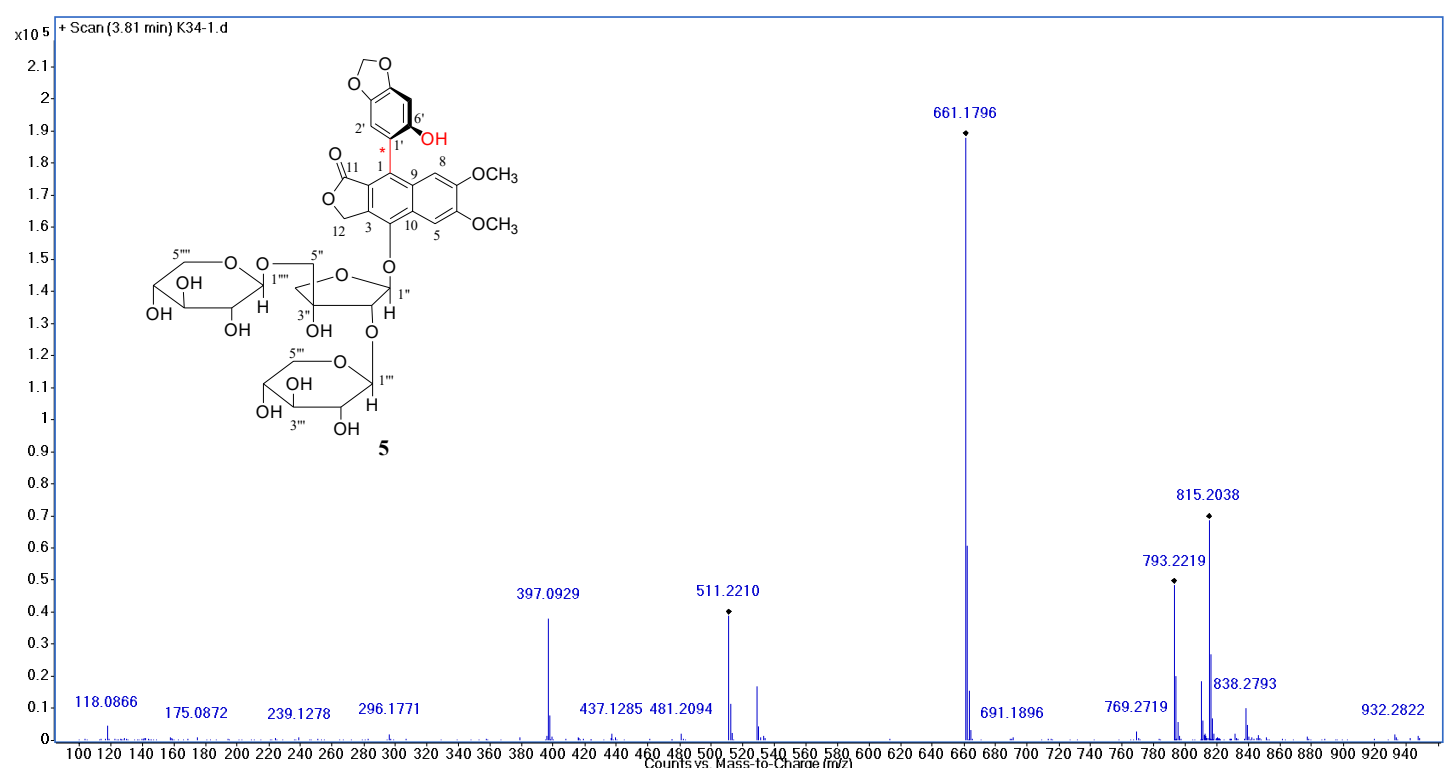

Figure S42. HRESIMS Data of Compound 5. 


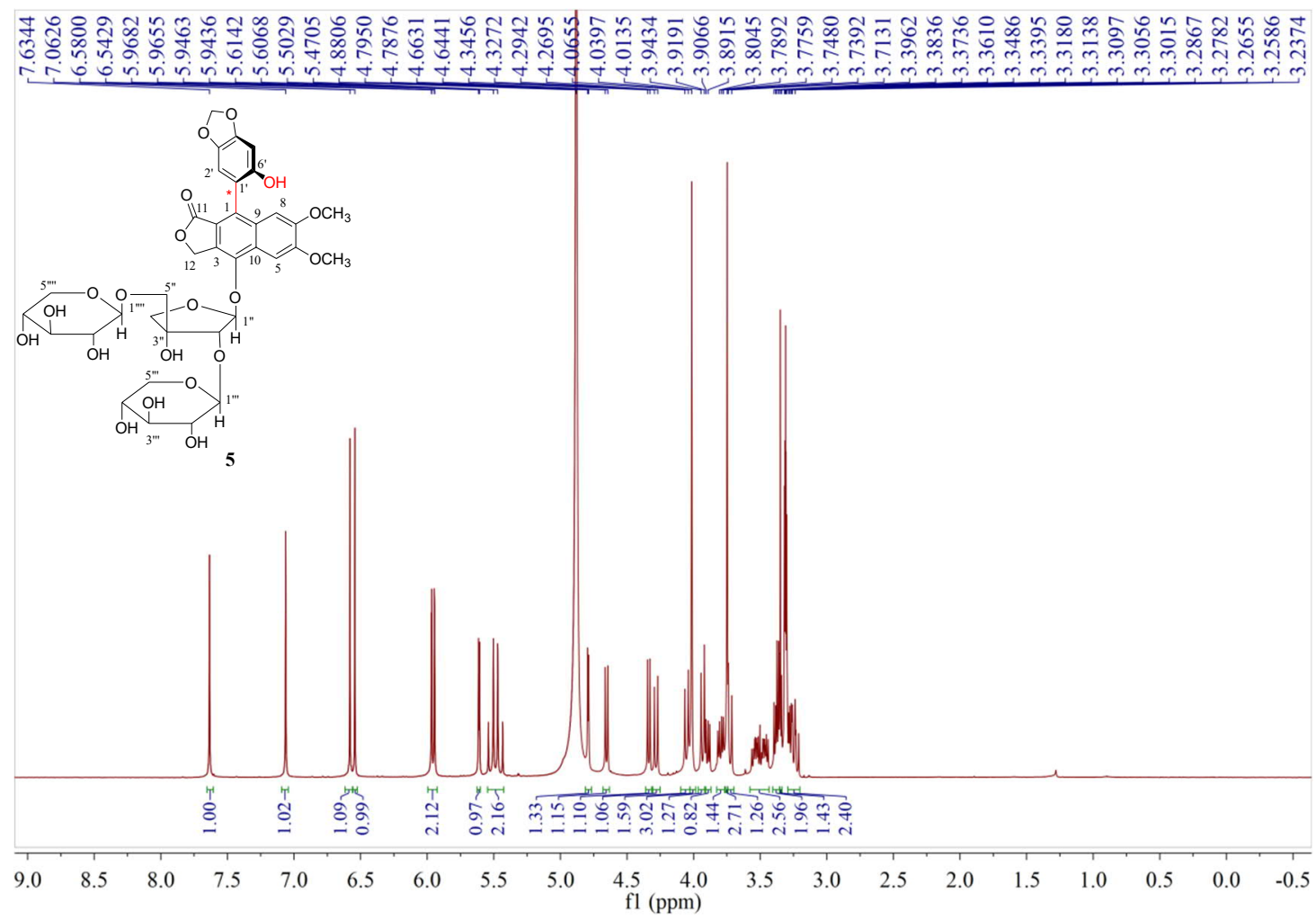

Figure S43. The ${ }^{1} \mathrm{H}$ NMR Spectrum of Compound 5 in $\mathrm{CD}_{3} \mathrm{OD}(400 \mathrm{MHz})$.

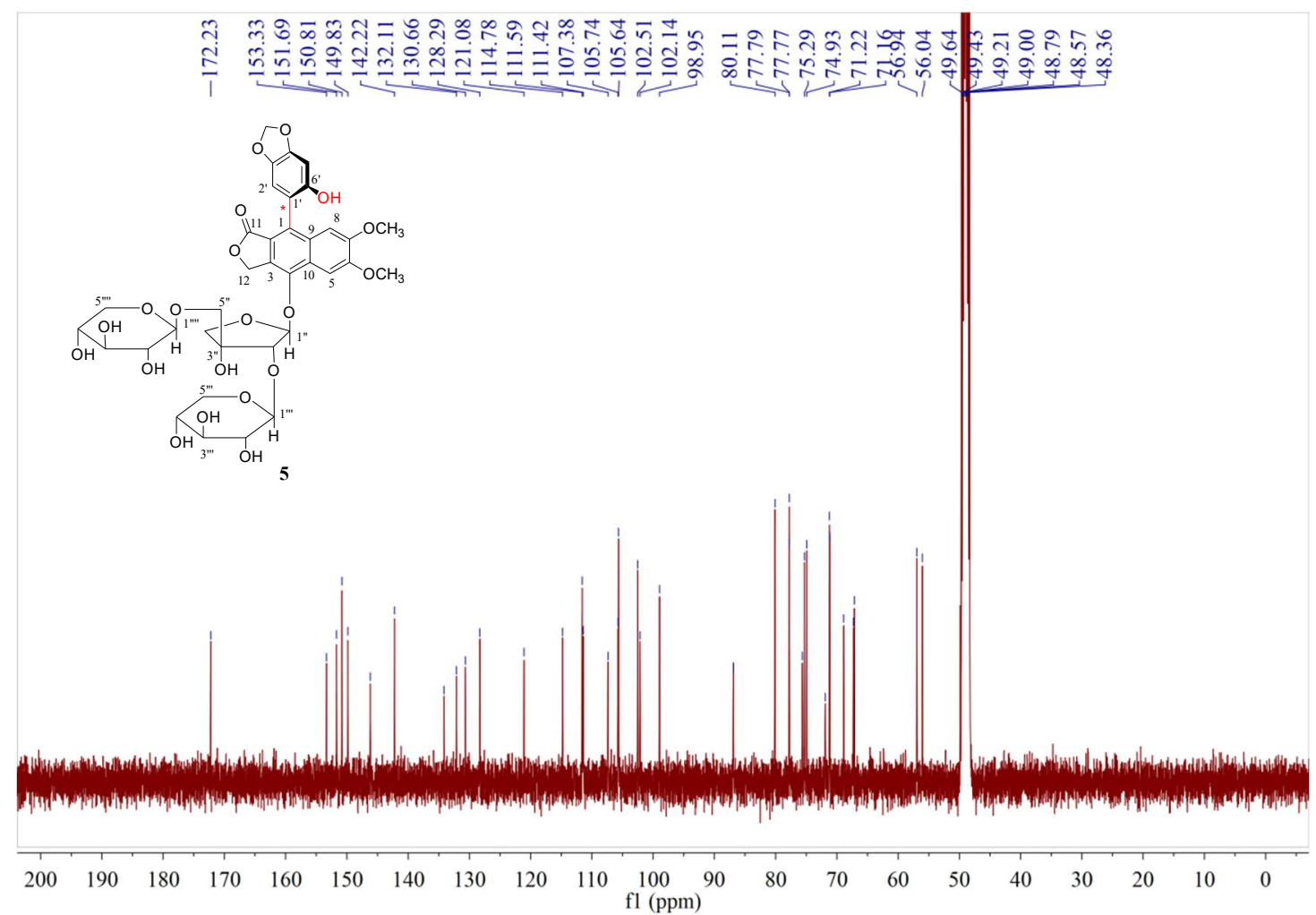

Figure S44. The ${ }^{13} \mathrm{C}\left\{{ }^{1} \mathrm{H}\right\}$ NMR Spectrum of Compound 5 in $\mathrm{CD}_{3} \mathrm{OD}(100 \mathrm{MHz})$. 


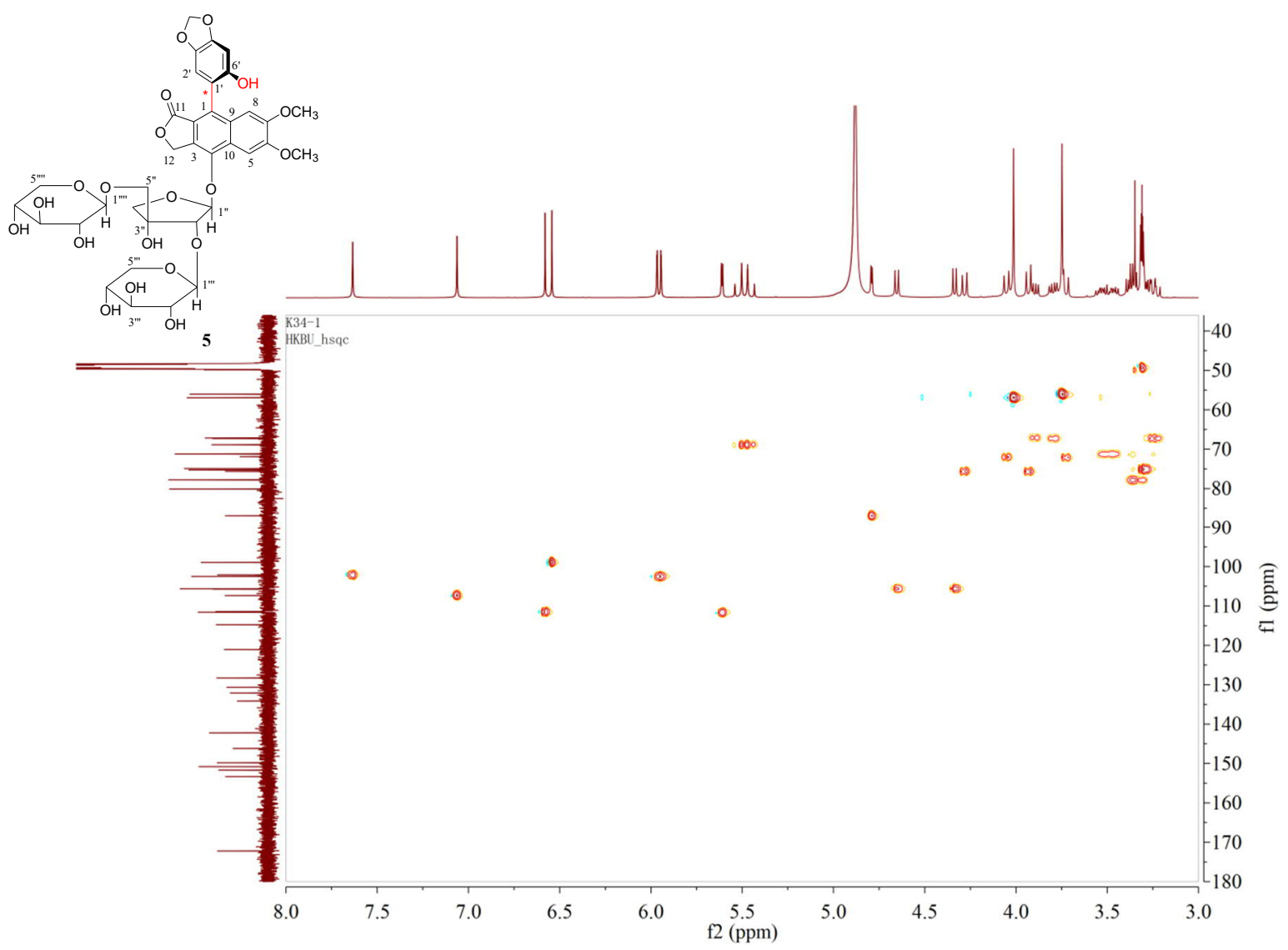

Figure S45. The HSQC Spectrum of Compound 5 in $\mathrm{CD}_{3} \mathrm{OD}\left(400 \mathrm{MHz}\right.$ for $\left.{ }^{1} \mathrm{H}\right)$.

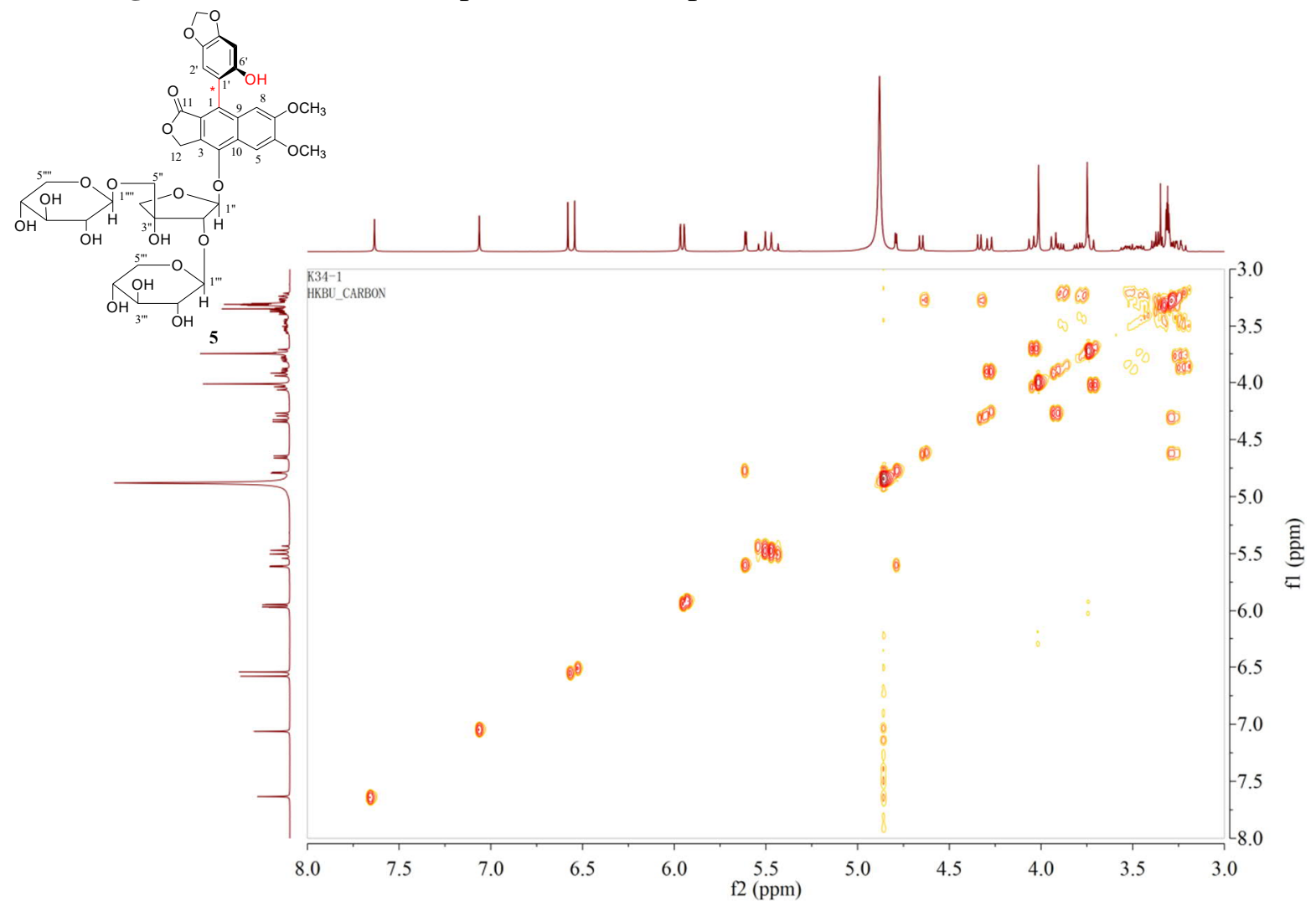

Figure S46. The ${ }^{1} \mathrm{H}-{ }^{1} \mathrm{H}$ COSY Spectrum of Compound 5 in $\mathrm{CD}_{3} \mathrm{OD}(400 \mathrm{MHz})$. 


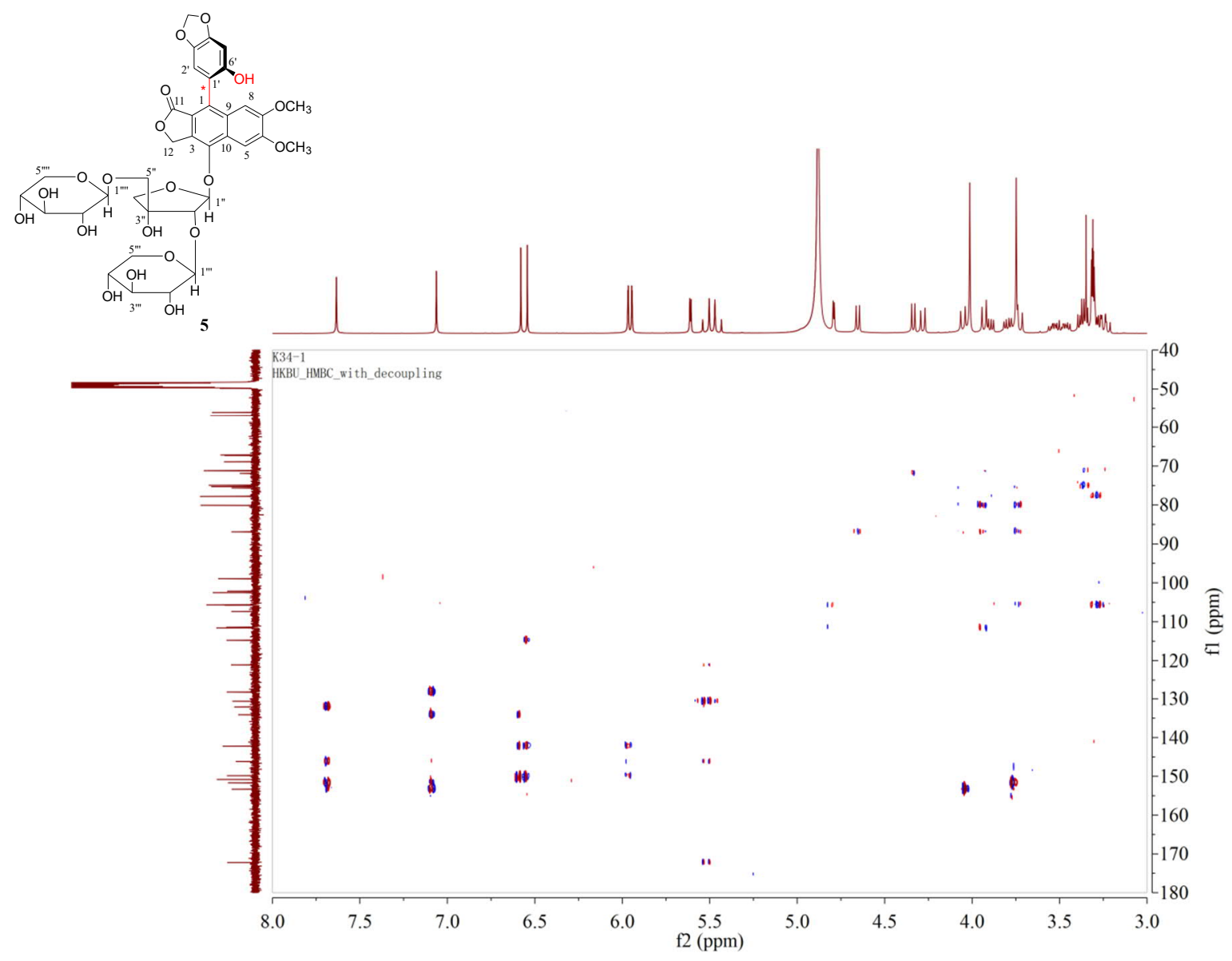

Figure S47. The HMBC Spectrum of Compound 5 in $\mathrm{CD}_{3} \mathrm{OD}\left(400 \mathrm{MHz}\right.$ for $\left.{ }^{1} \mathrm{H}\right)$.

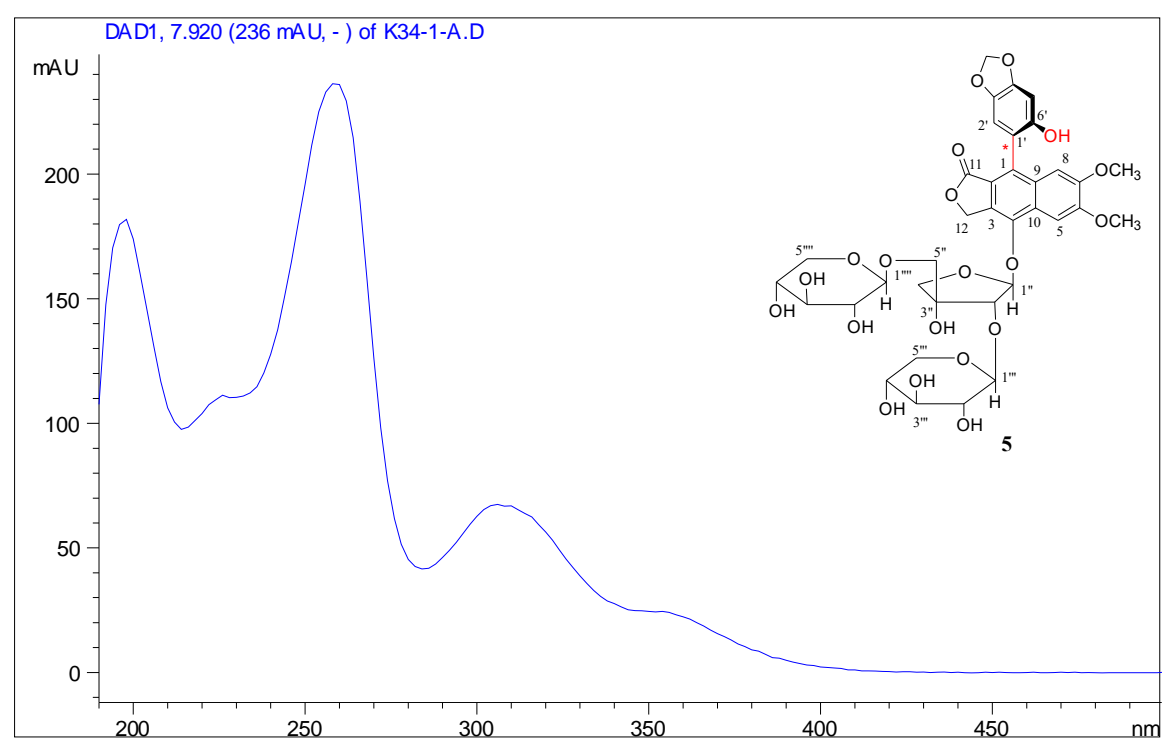

Figure S48. The UV Spectrum of Compound 5. 


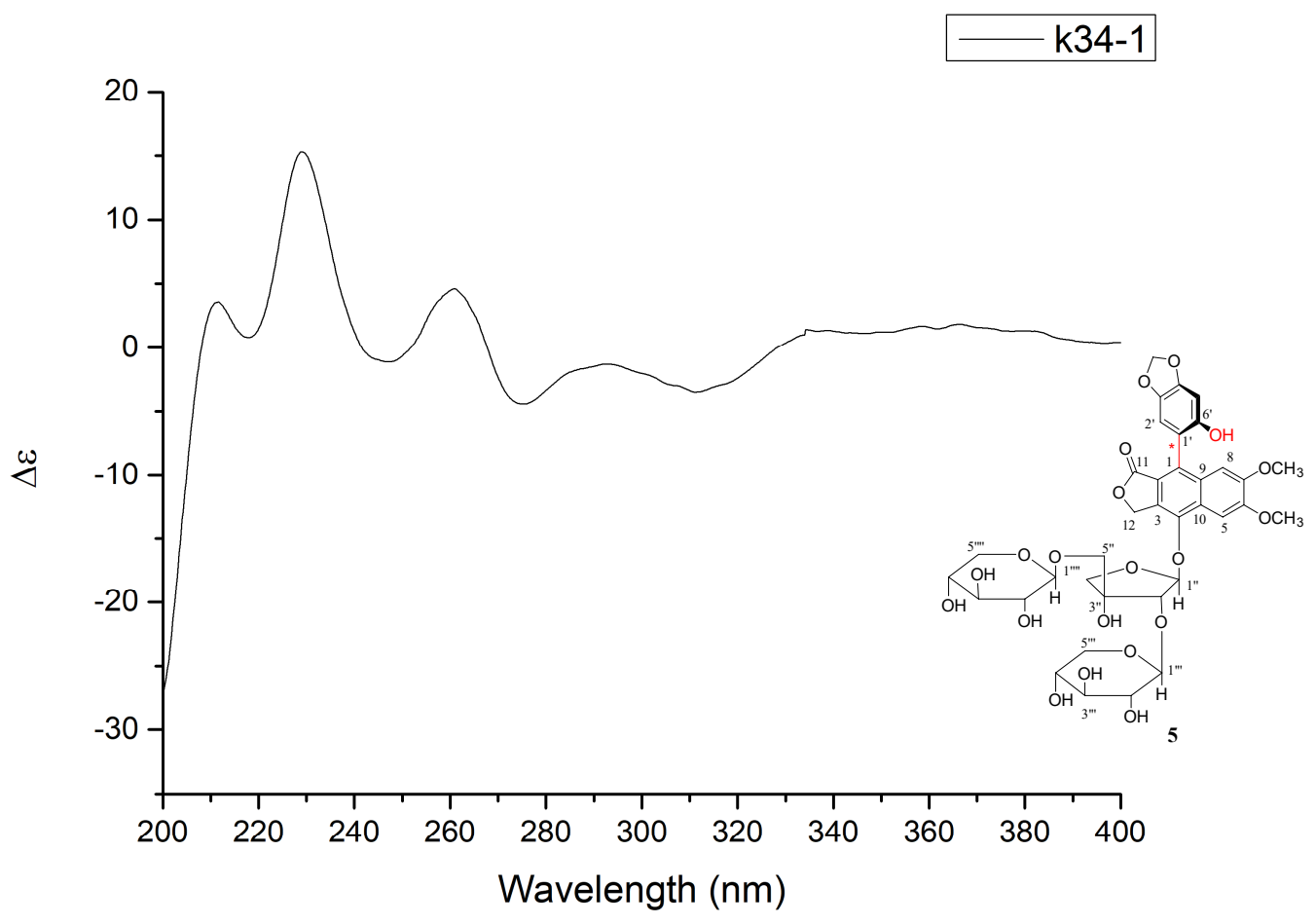

Figure S49. The CD Spectrum of Compound 5.

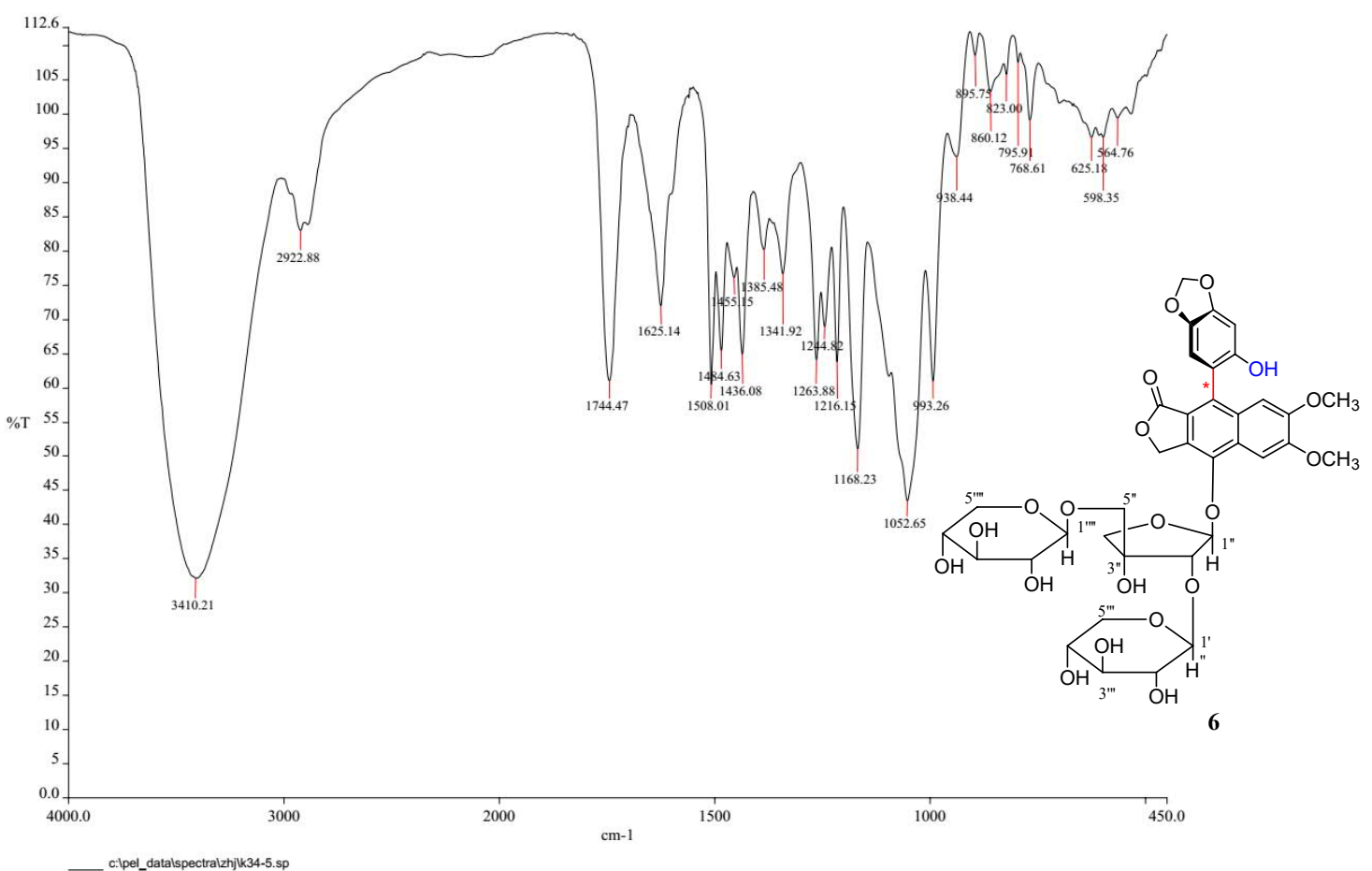

Figure S50. The IR Spectrum of Compound 6. 


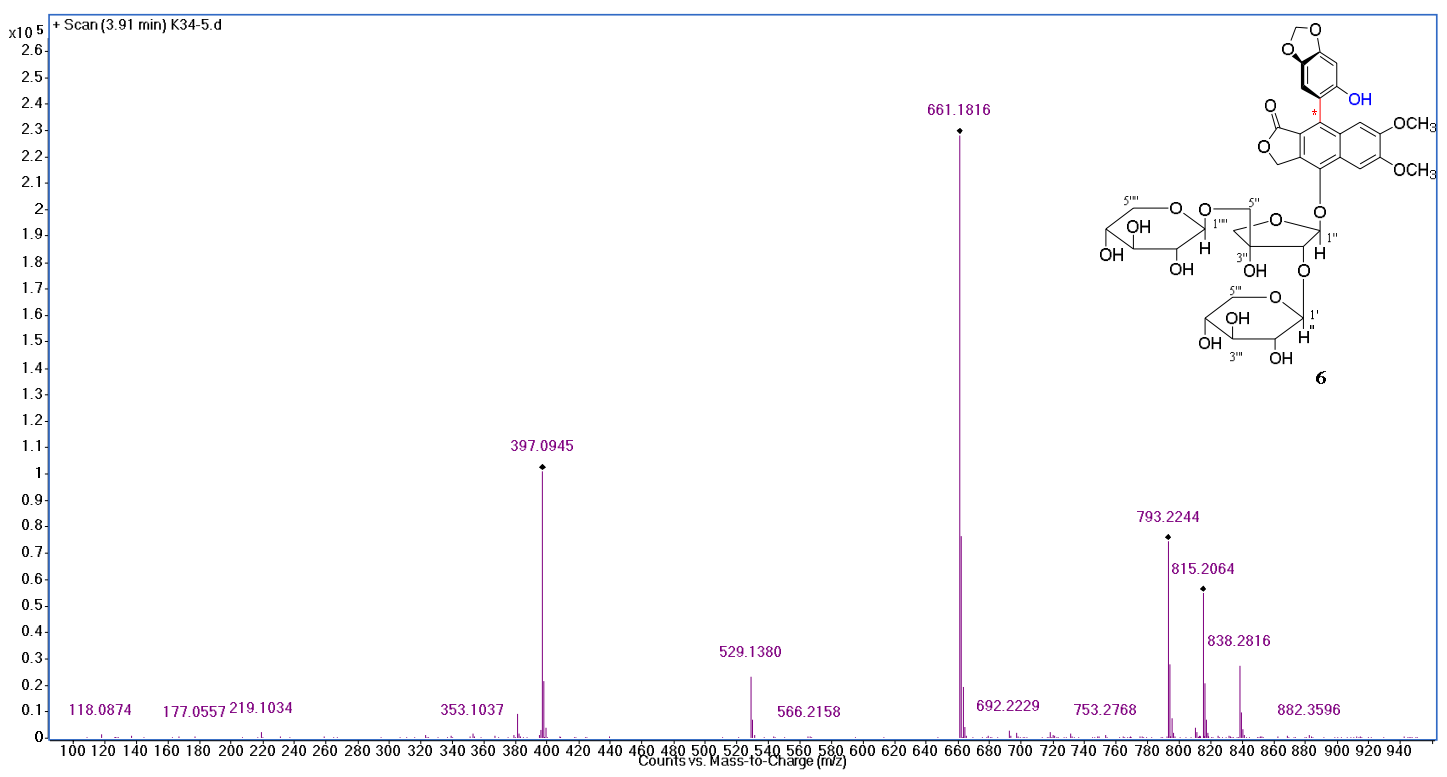

Figure S51. HRESIMS Data of Compound 6.

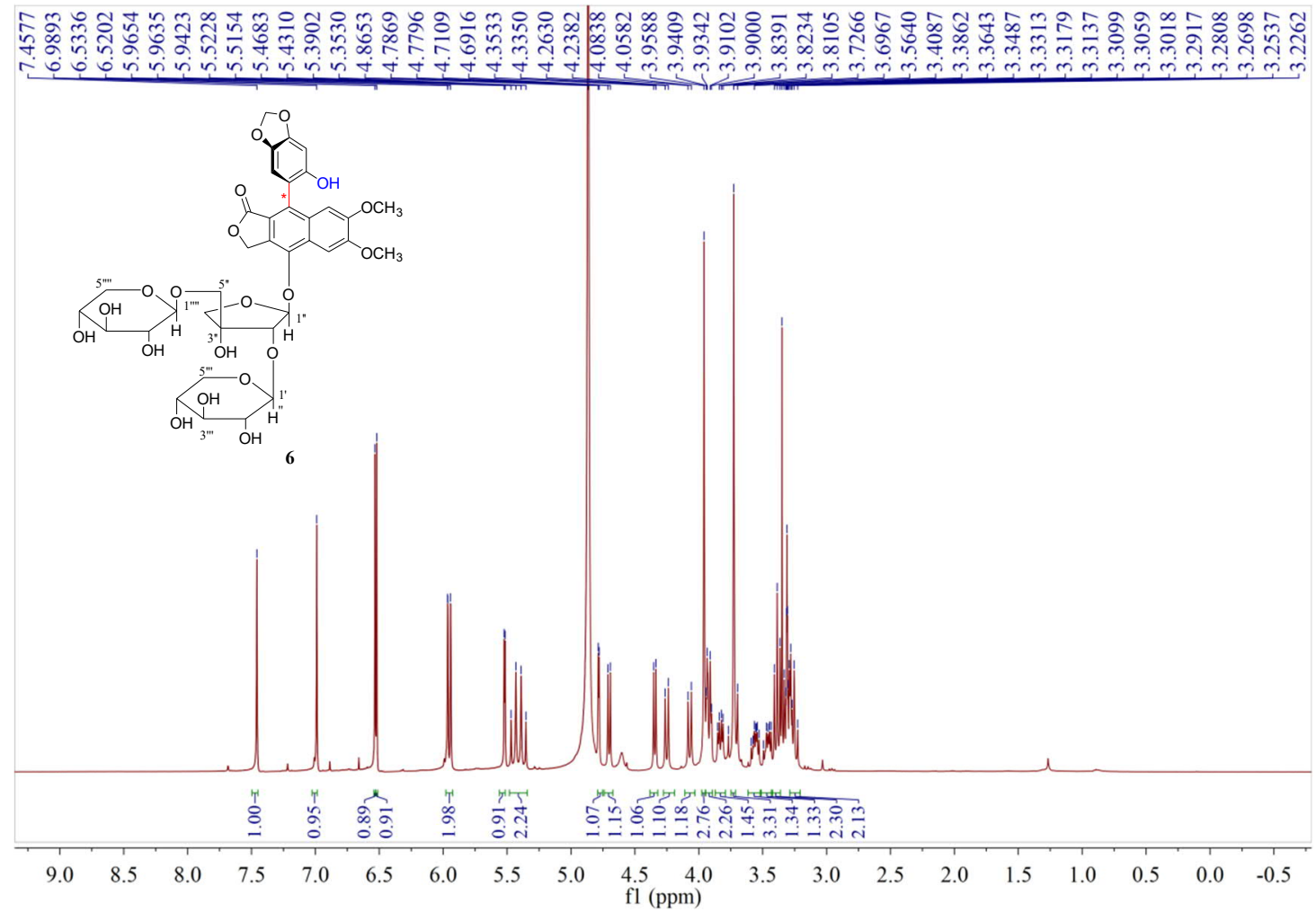

Figure S52. The ${ }^{1} \mathrm{H}$ NMR Spectrum of Compound 6 in $\mathrm{CD}_{3} \mathrm{OD}(400 \mathrm{MHz})$. 


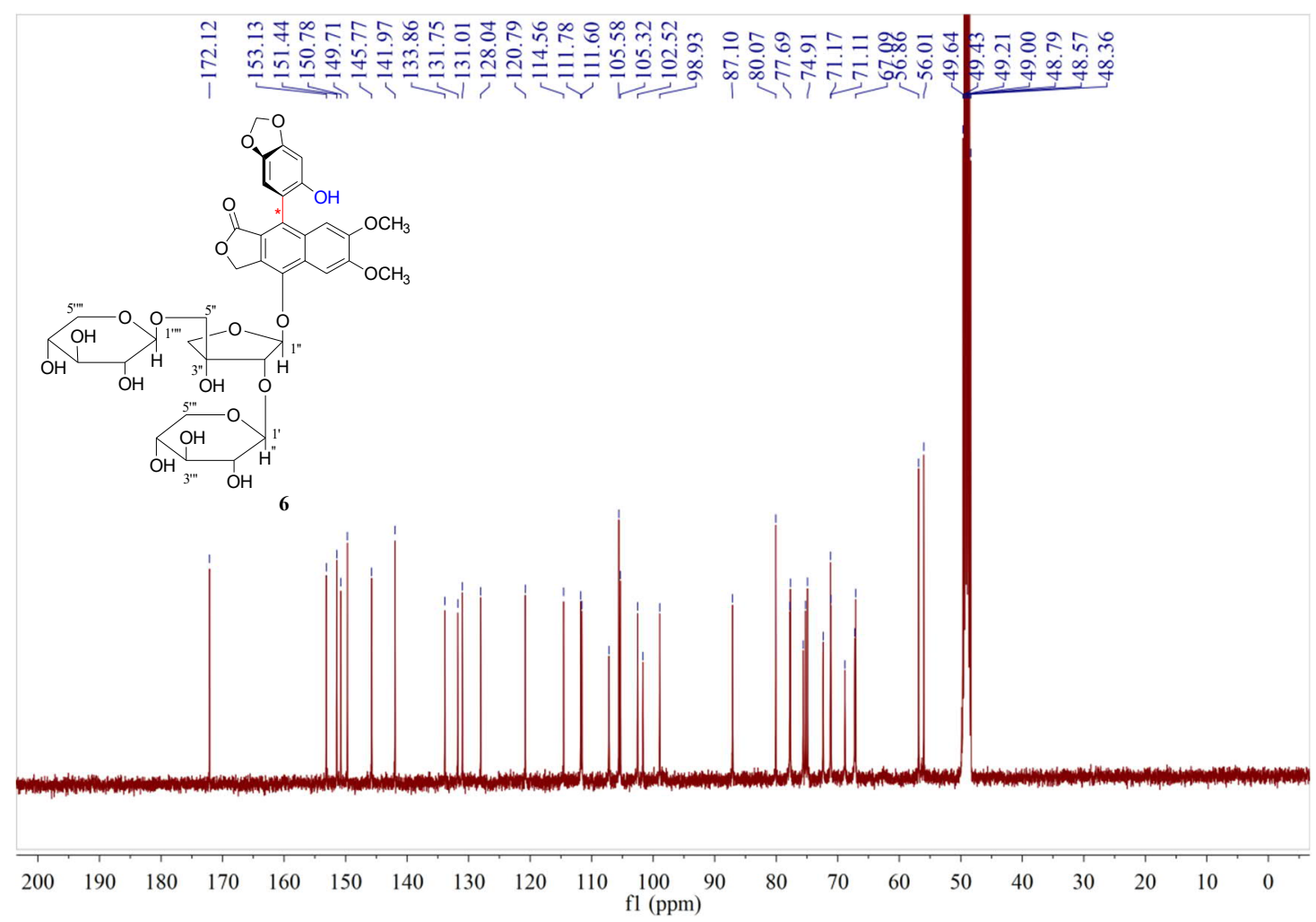

Figure S53. The ${ }^{13} \mathrm{C}\left\{{ }^{1} \mathrm{H}\right\}$ NMR Spectrum of Compound 6 in $\mathrm{CD}_{3} \mathrm{OD}(100 \mathrm{MHz})$.

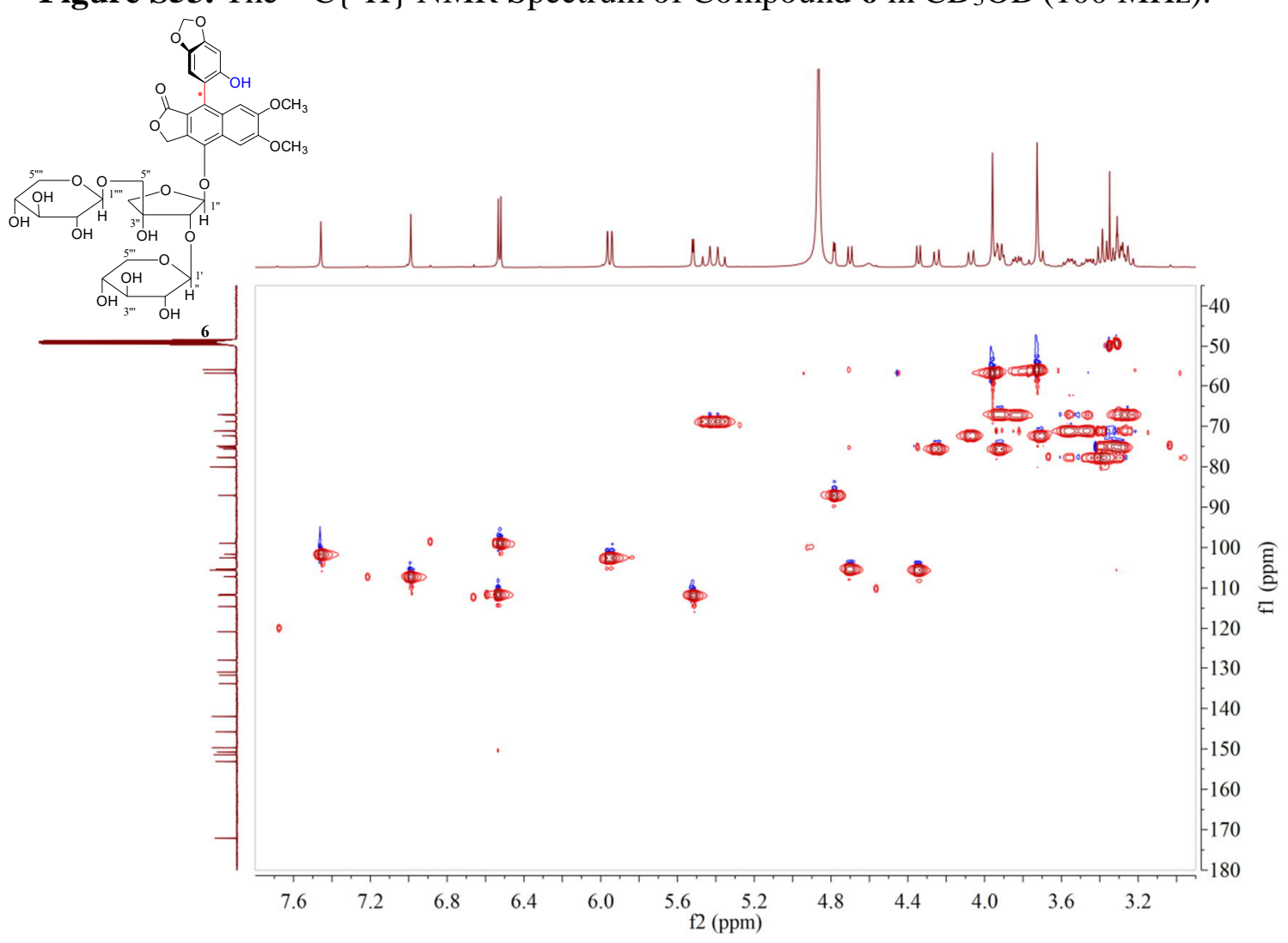

Figure S54. The HSQC Spectrum of Compound 6 in $\mathrm{CD}_{3} \mathrm{OD}\left(400 \mathrm{MHz}\right.$ for $\left.{ }^{1} \mathrm{H}\right)$. 


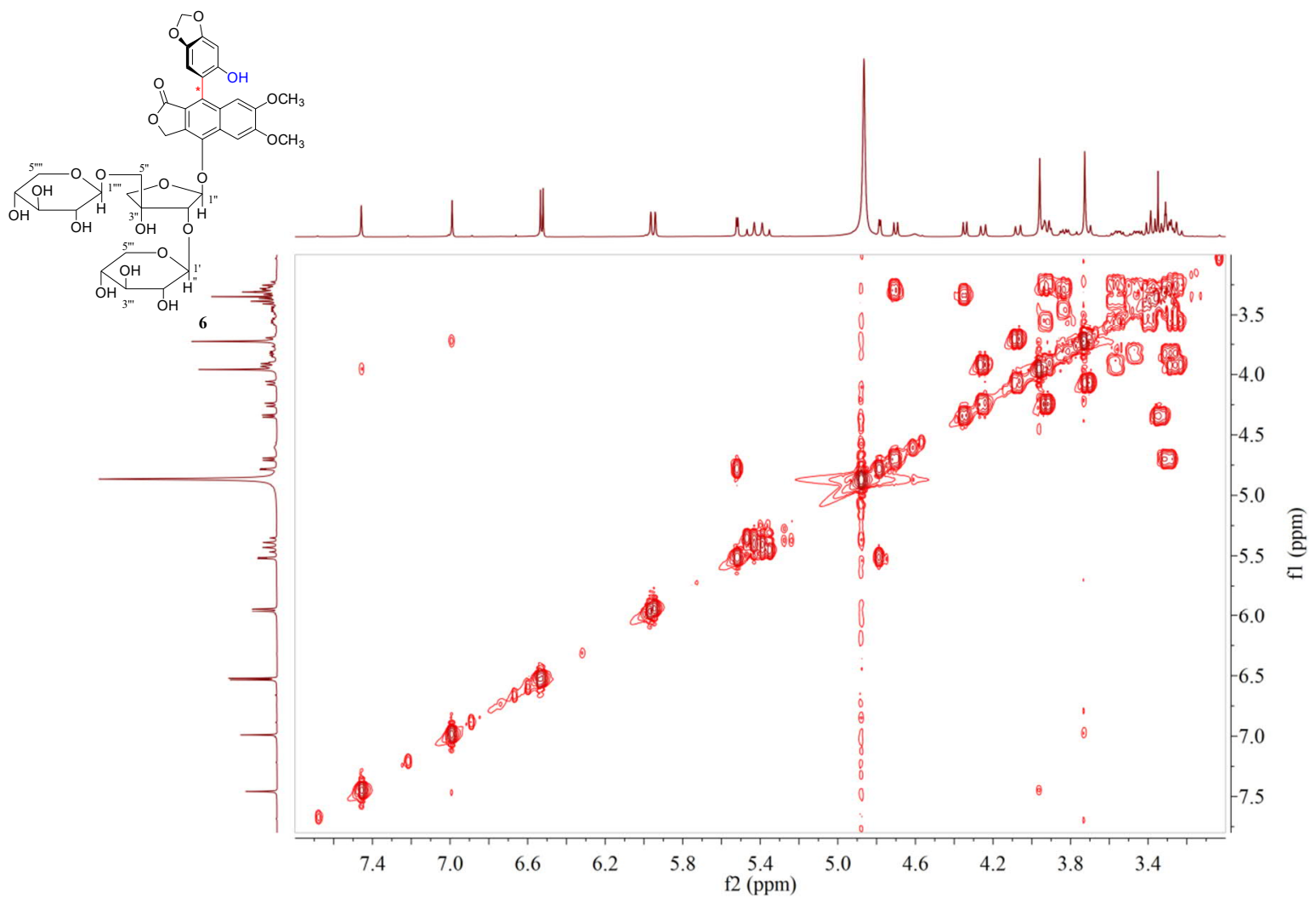

Figure S55. The ${ }^{1} \mathrm{H}-{ }^{1} \mathrm{H}$ COSY Spectrum of Compound 6 in $\mathrm{CD}_{3} \mathrm{OD}(400 \mathrm{MHz})$.

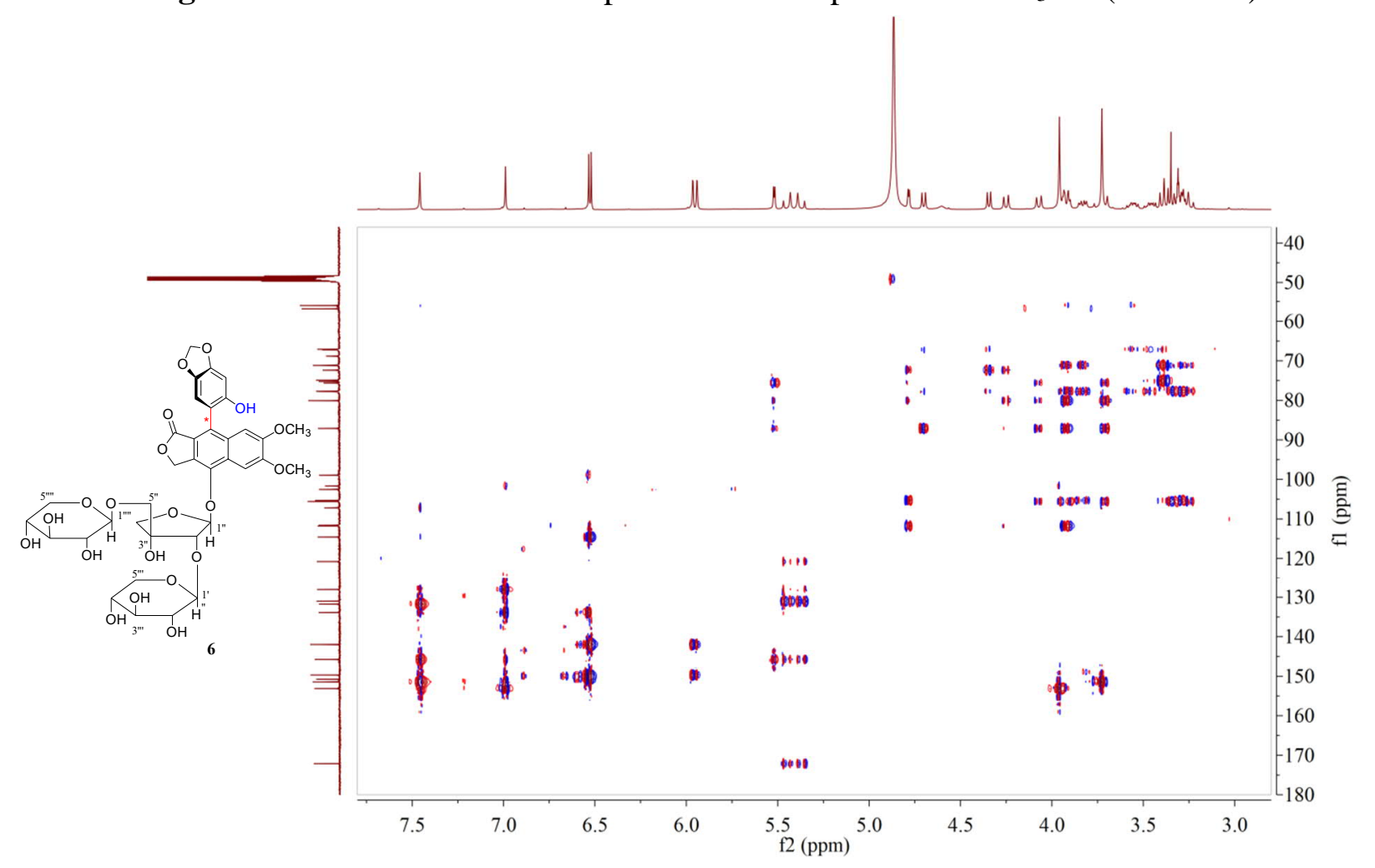

Figure S56. The HMBC Spectrum of Compound 6 in $\mathrm{CD}_{3} \mathrm{OD}\left(400 \mathrm{MHz}\right.$ for $\left.{ }^{1} \mathrm{H}\right)$. 


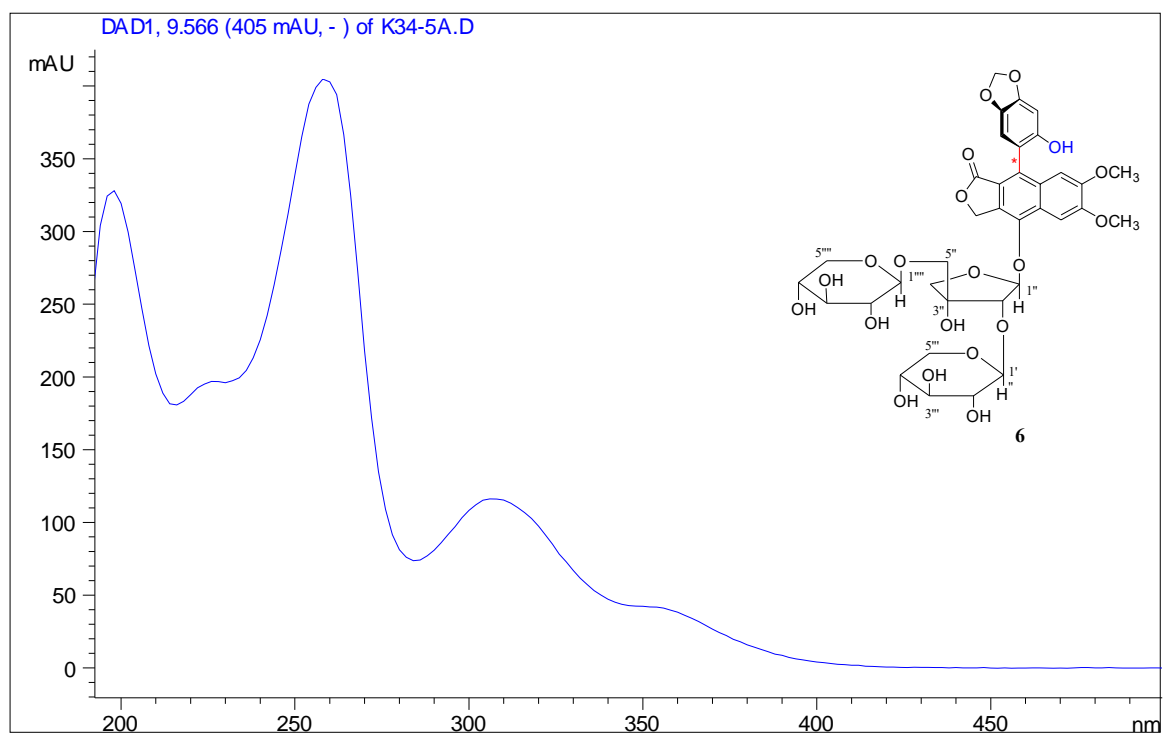

Figure S57. The UV Spectrum of Compound 6.

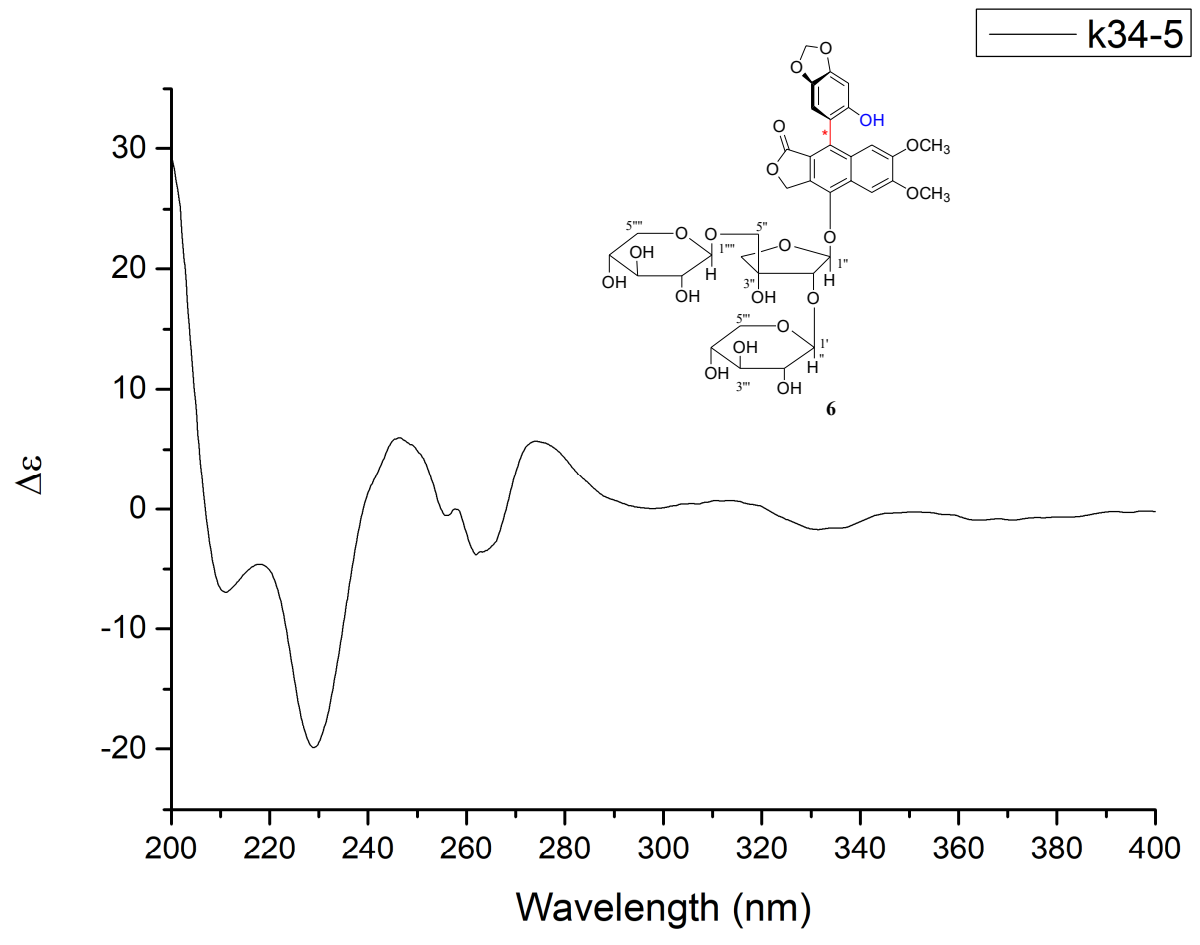

Figure S58. The CD Spectrum of Compound 6. 


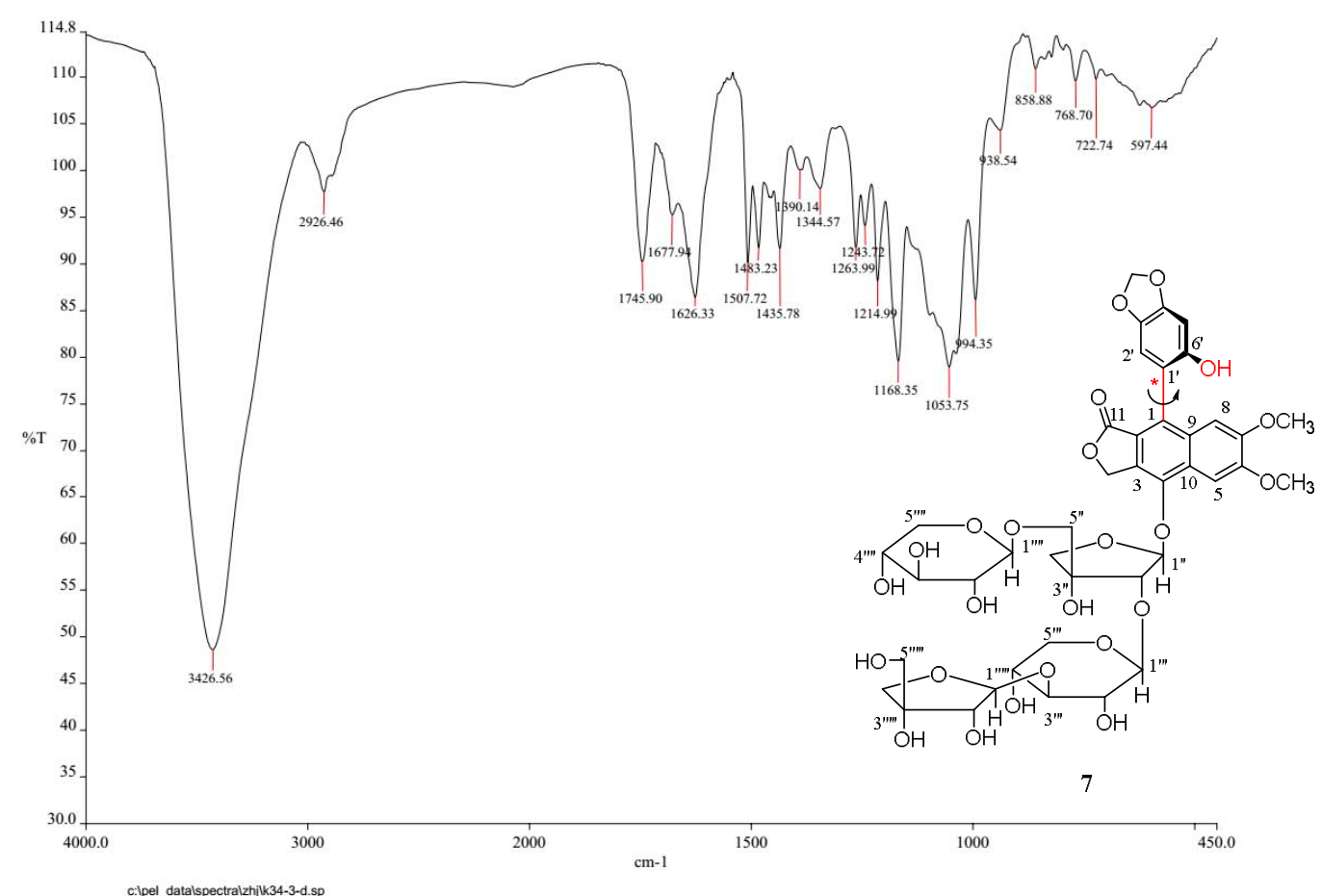

Figure S59. The IR Spectrum of Compound 7.

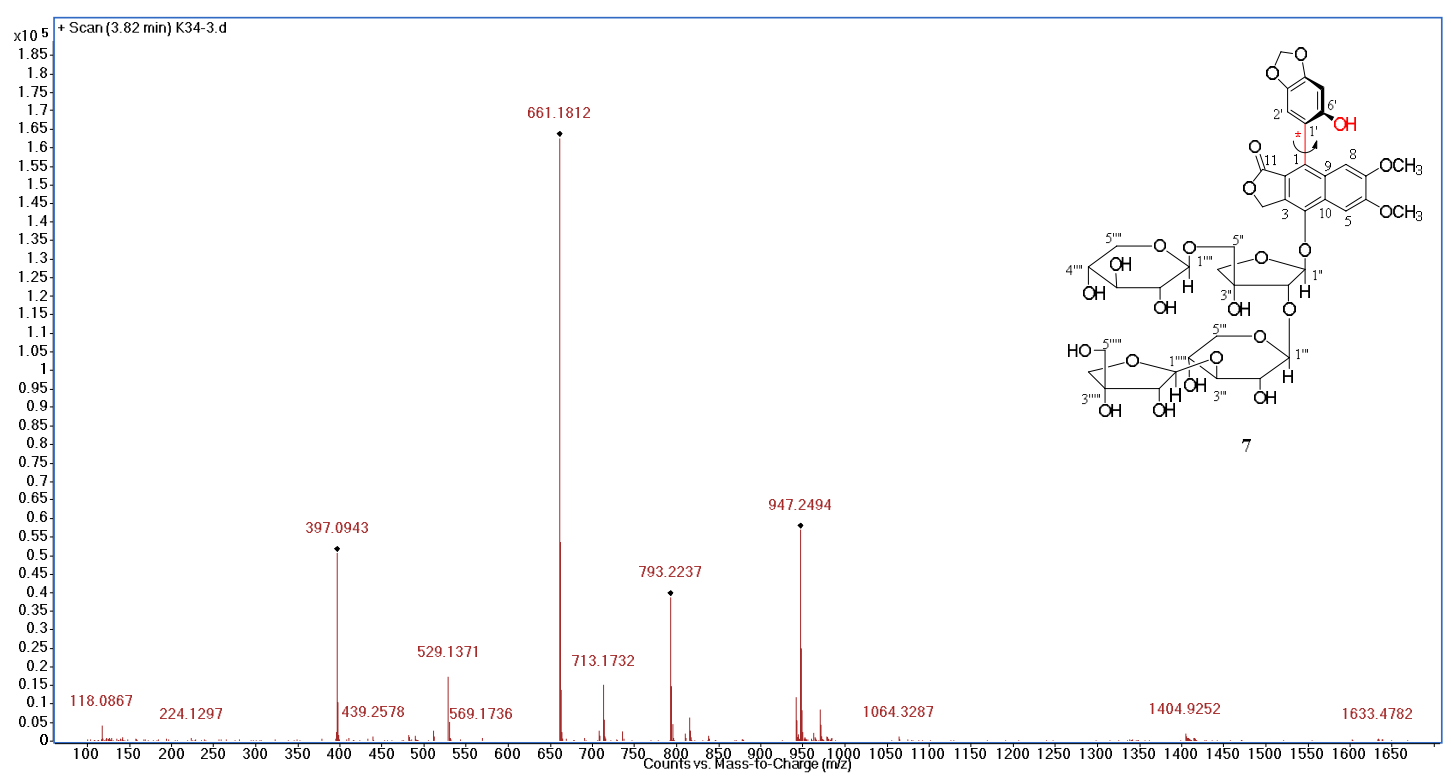

Figure S60. HRESIMS Data of Compound 7. 


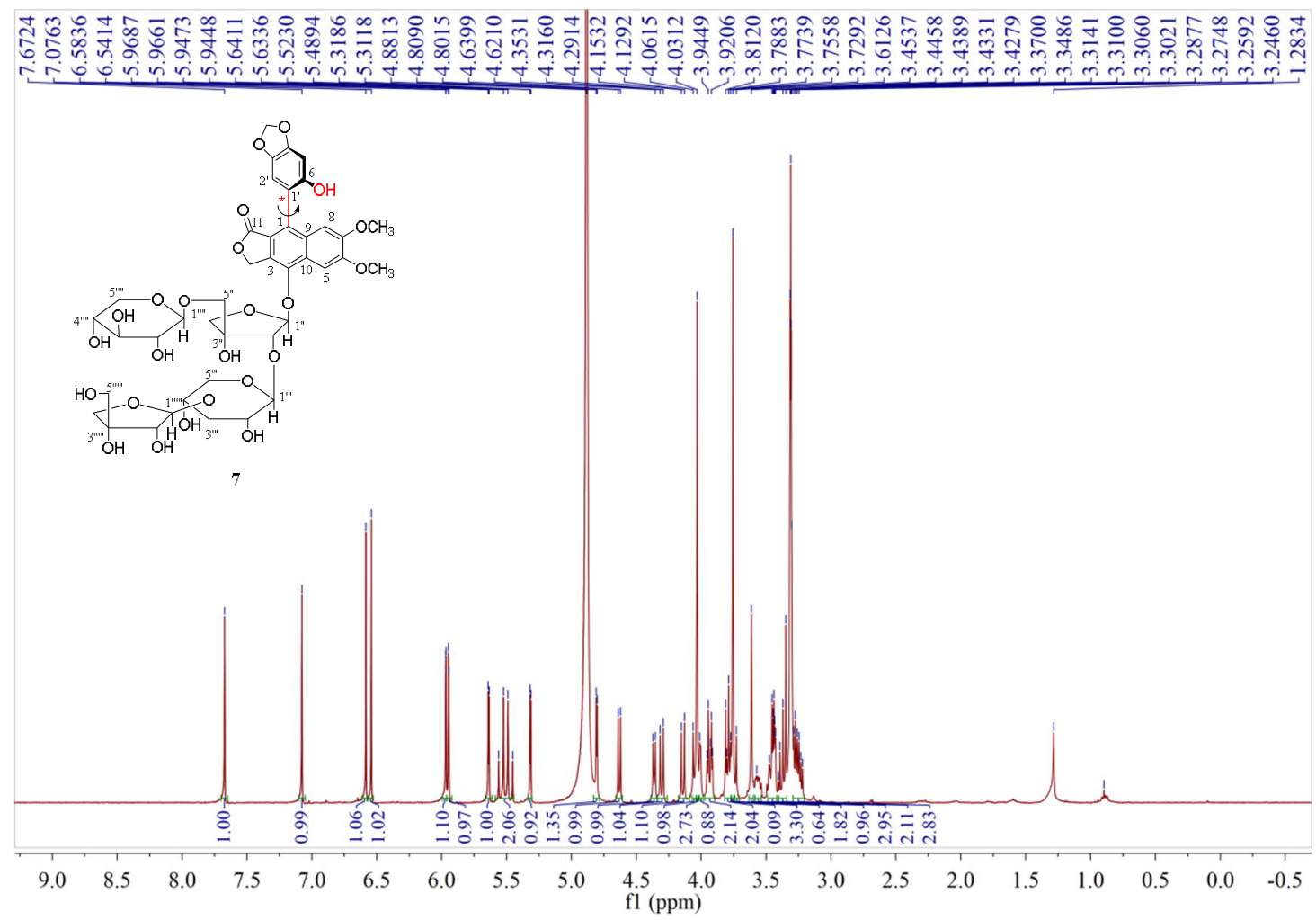

Figure S61. The ${ }^{1} \mathrm{H}$ NMR Spectrum of Compound 7 in $\mathrm{CD}_{3} \mathrm{OD}(400 \mathrm{MHz})$.

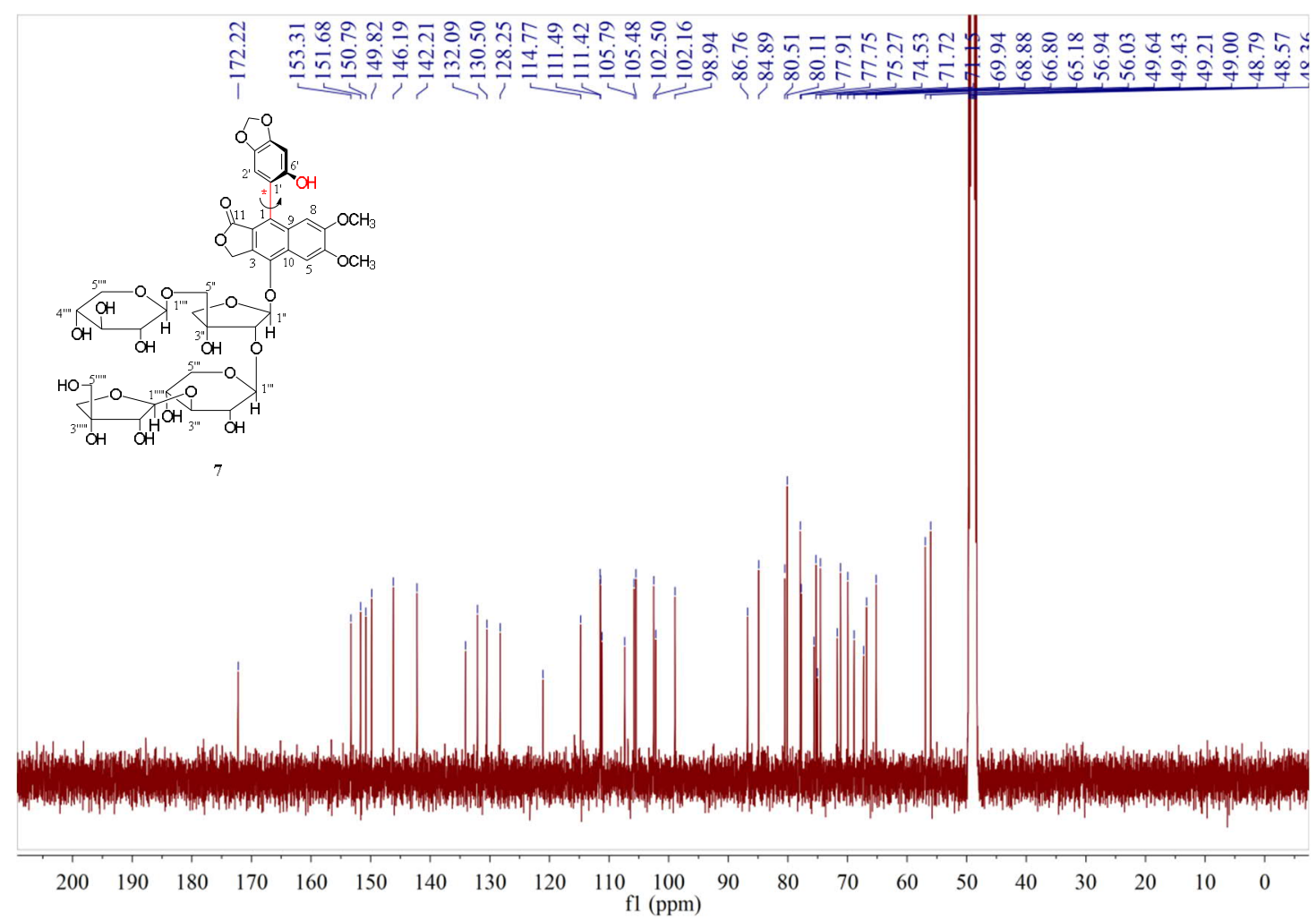

Figure S62. The ${ }^{13} \mathrm{C}\left\{{ }^{1} \mathrm{H}\right\}$ NMR Spectrum of Compound 7 in $\mathrm{CD}_{3} \mathrm{OD}(100 \mathrm{MHz})$. 


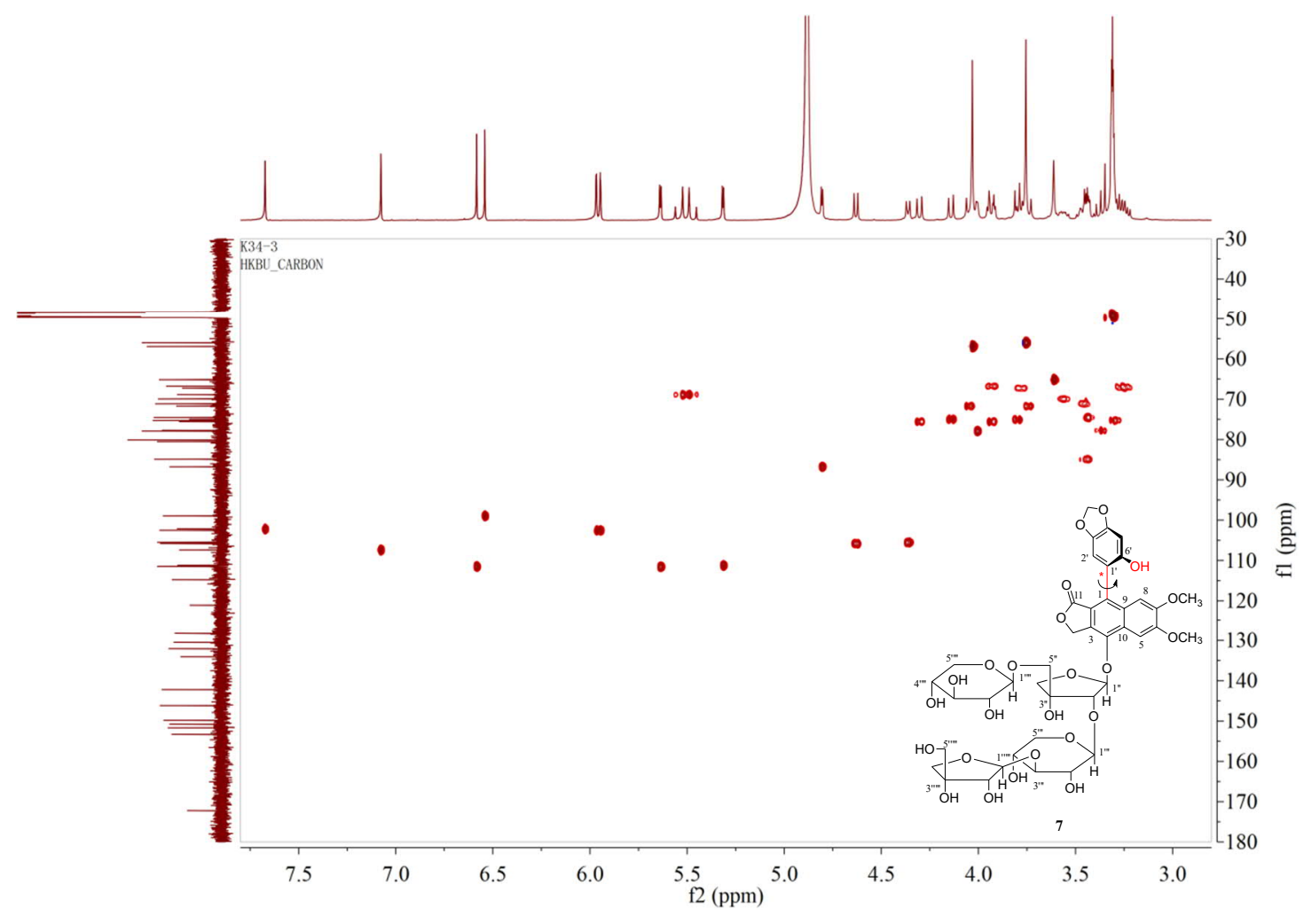

Figure S63. The HSQC Spectrum of Compound 7 in $\mathrm{CD}_{3} \mathrm{OD}\left(400 \mathrm{MHz}\right.$ for $\left.{ }^{1} \mathrm{H}\right)$.

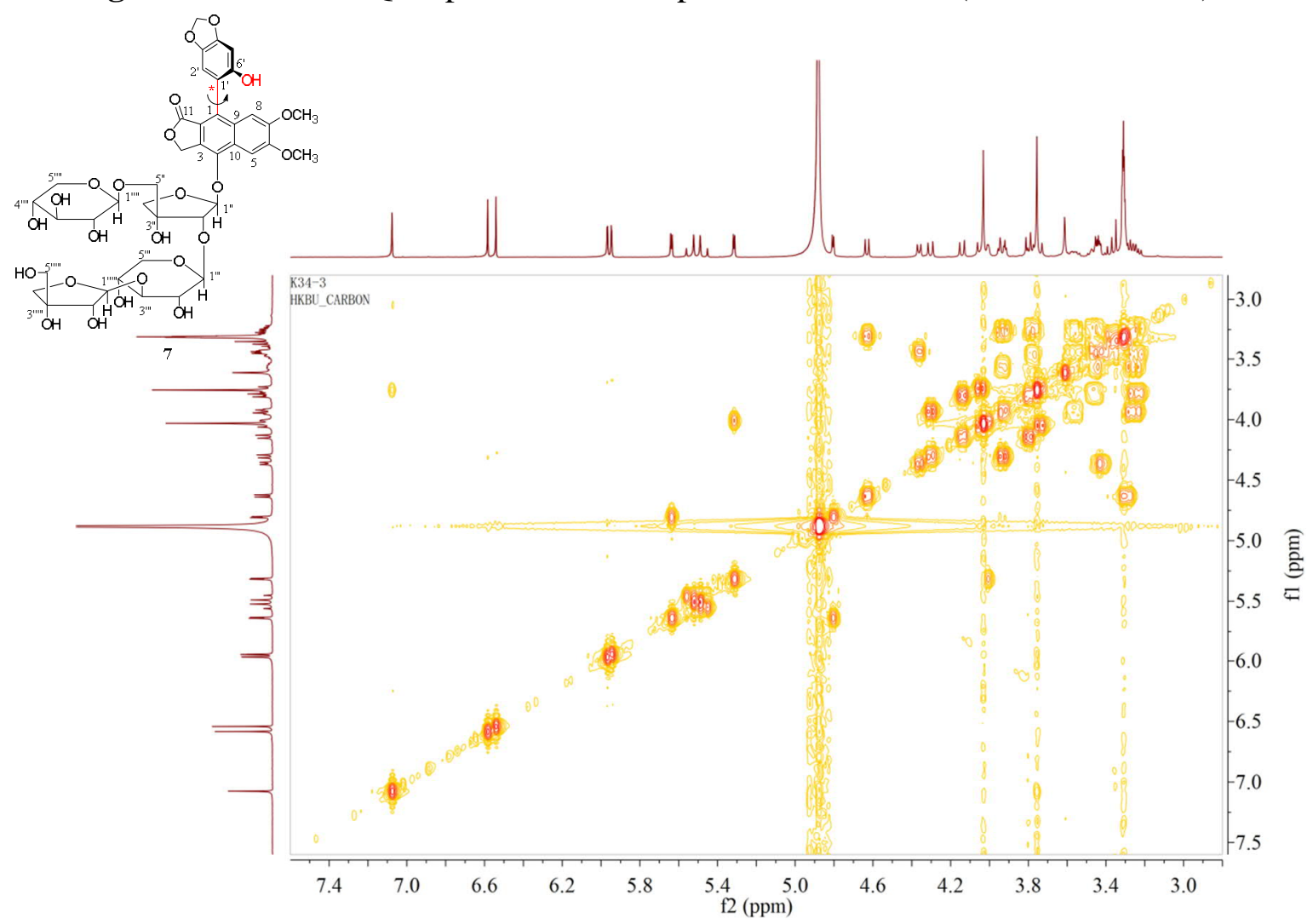

Figure S64. The ${ }^{1} \mathrm{H}_{-}{ }^{1} \mathrm{H}$ COSY Spectrum of Compound 7 in $\mathrm{CD}_{3} \mathrm{OD}(400 \mathrm{MHz})$. 


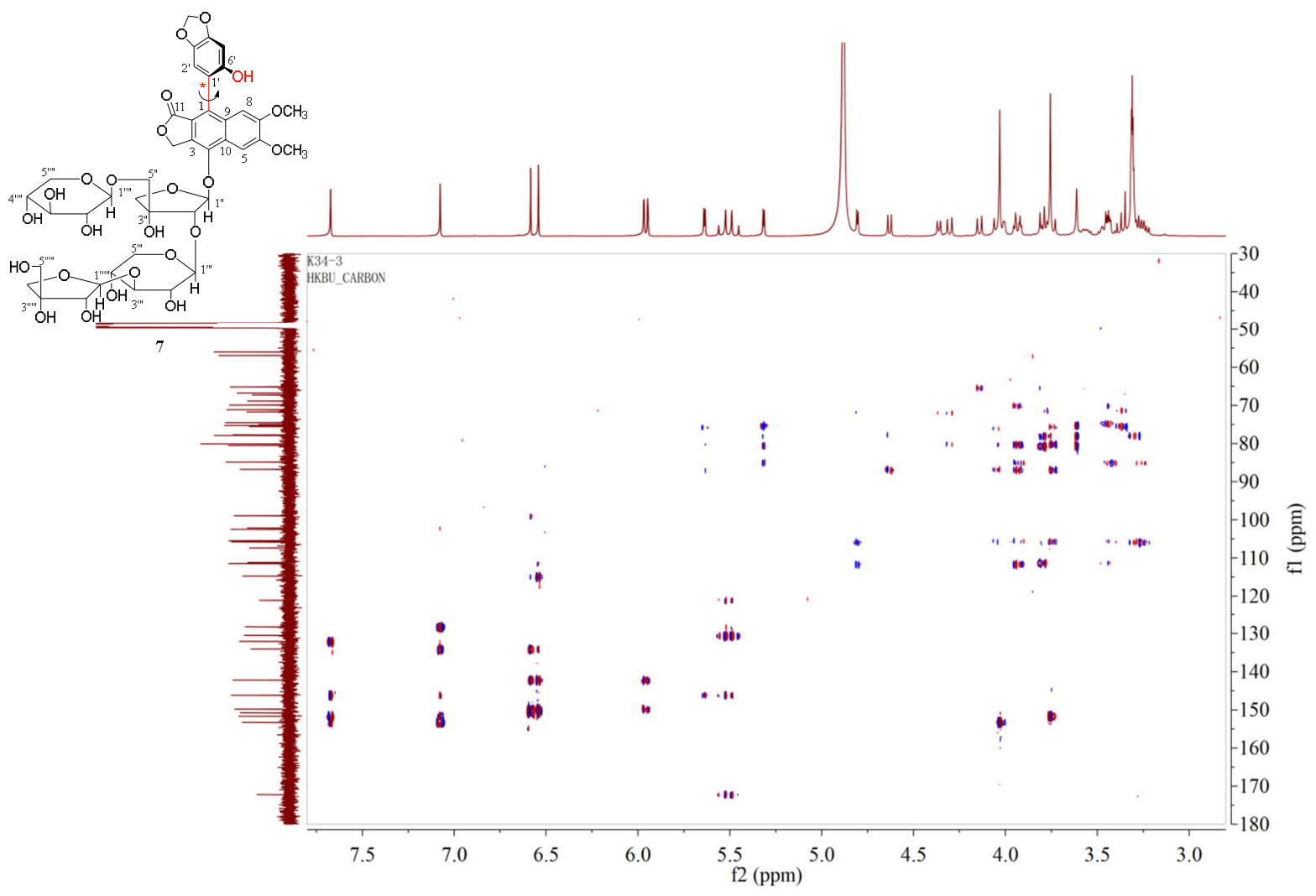

Figure S65. The HMBC Spectrum of Compound 7 in $\mathrm{CD}_{3} \mathrm{OD}\left(400 \mathrm{MHz}\right.$ for $\left.{ }^{1} \mathrm{H}\right)$.

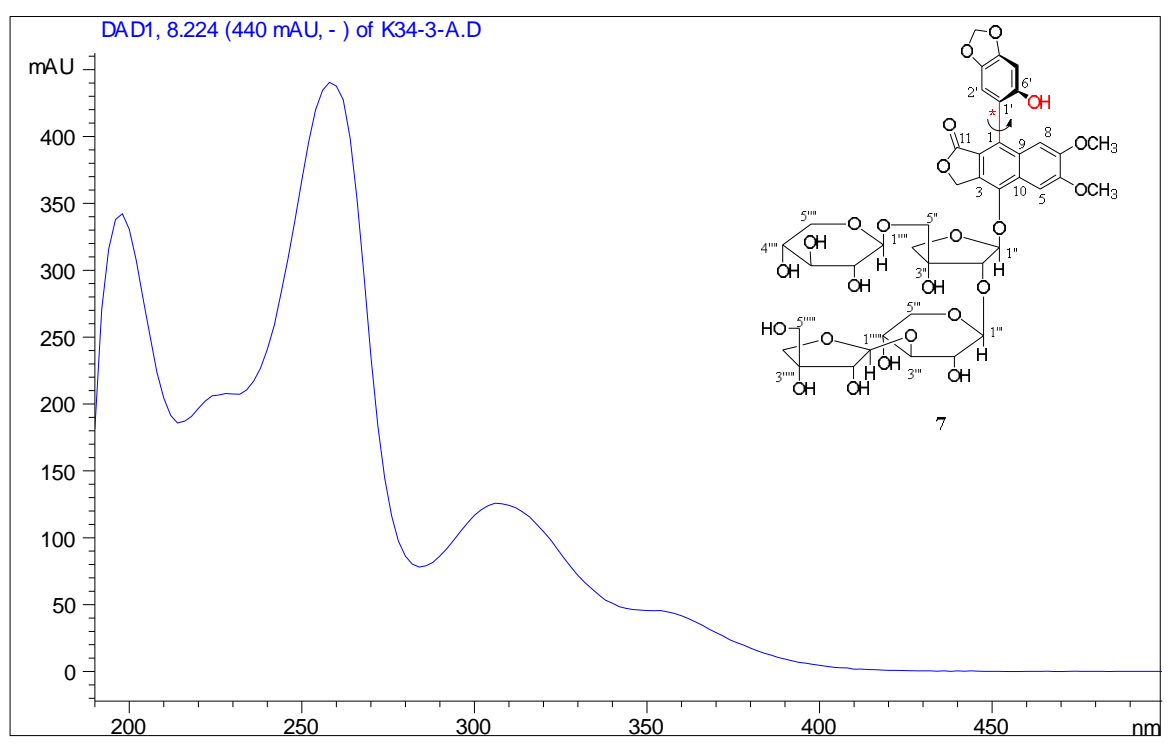

Figure S66. The UV Spectrum of Compound 7. 


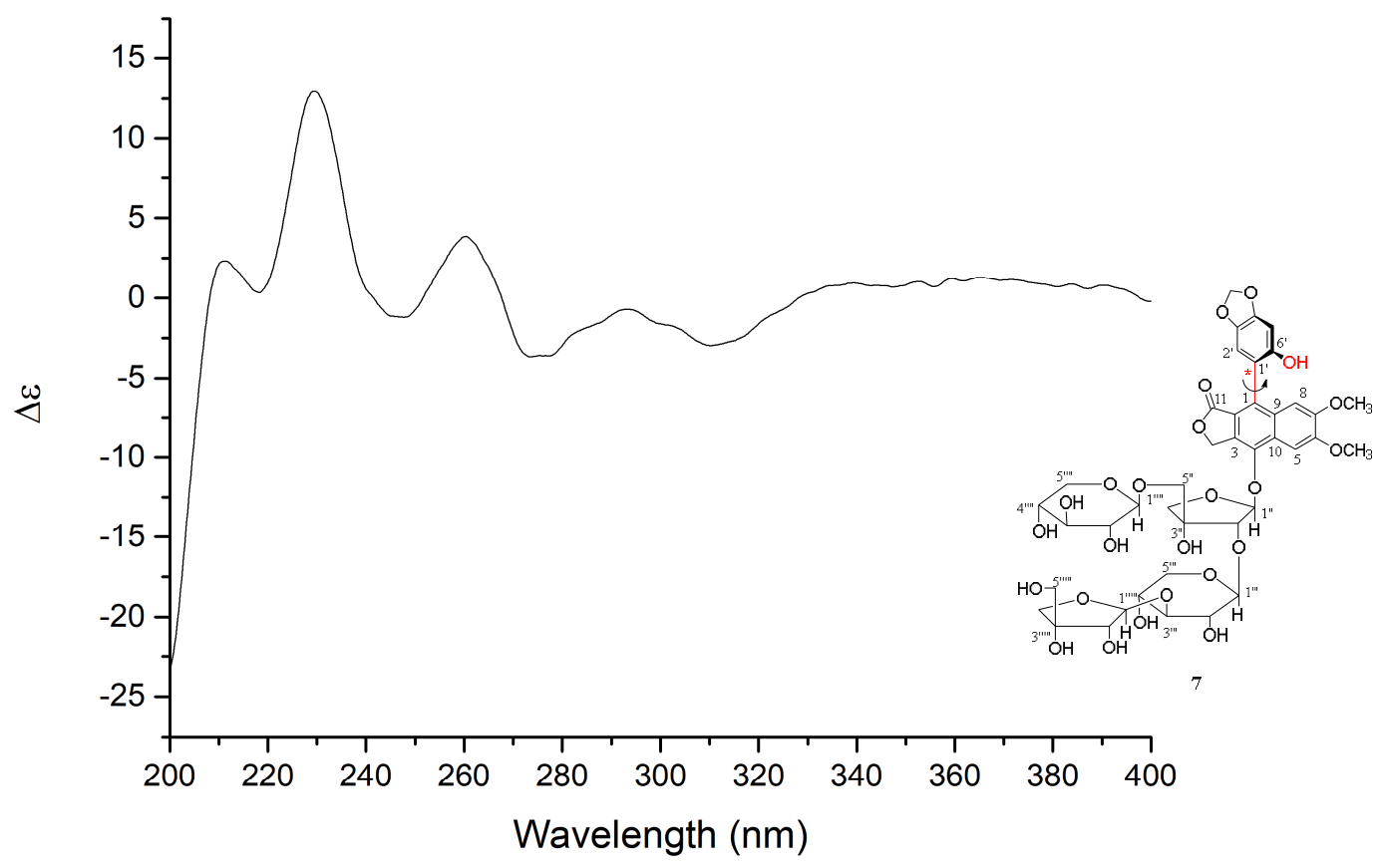

Figure S67. The CD Spectrum of Compound 7.

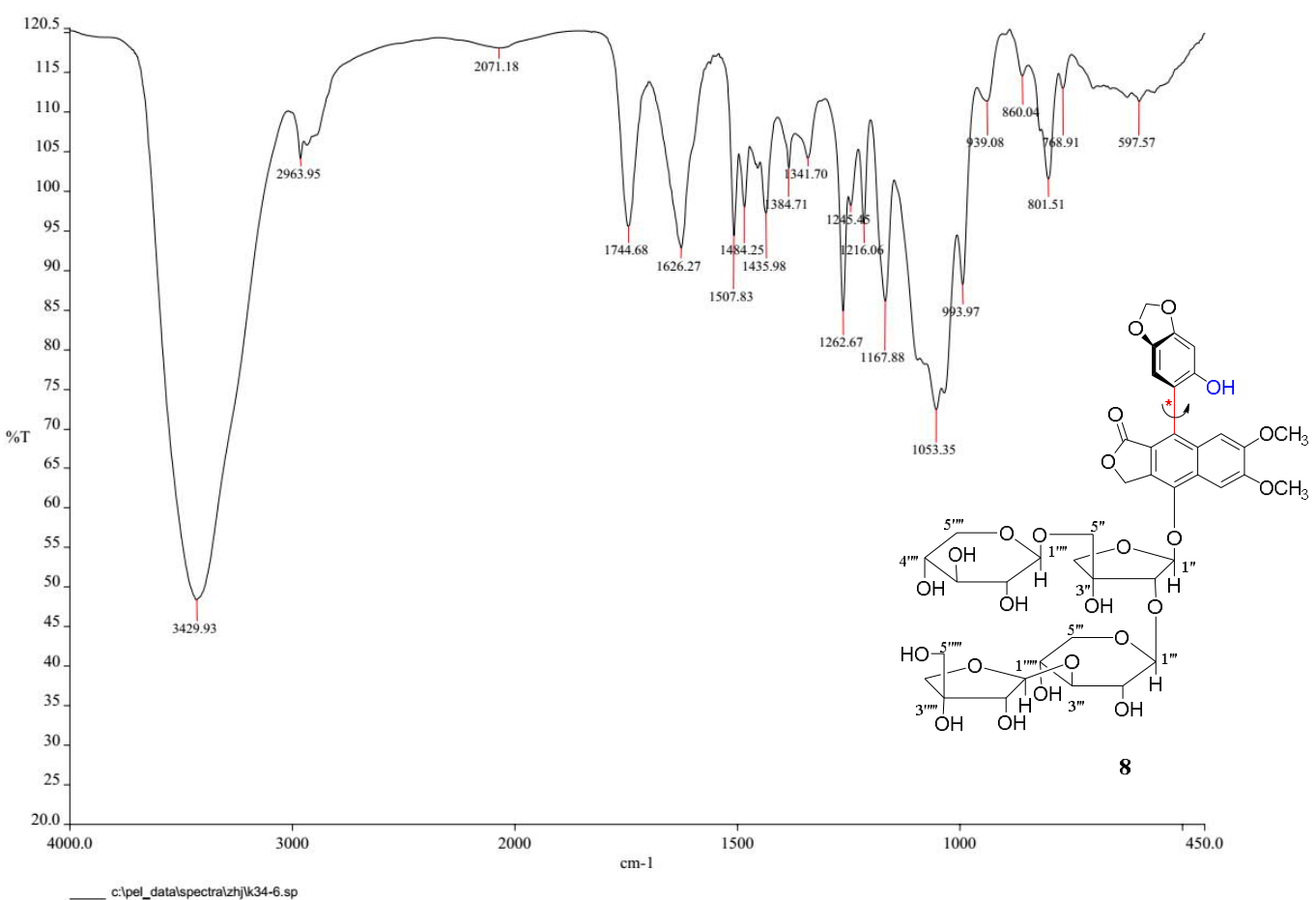

Figure S68. The IR Spectrum of Compound 8. 


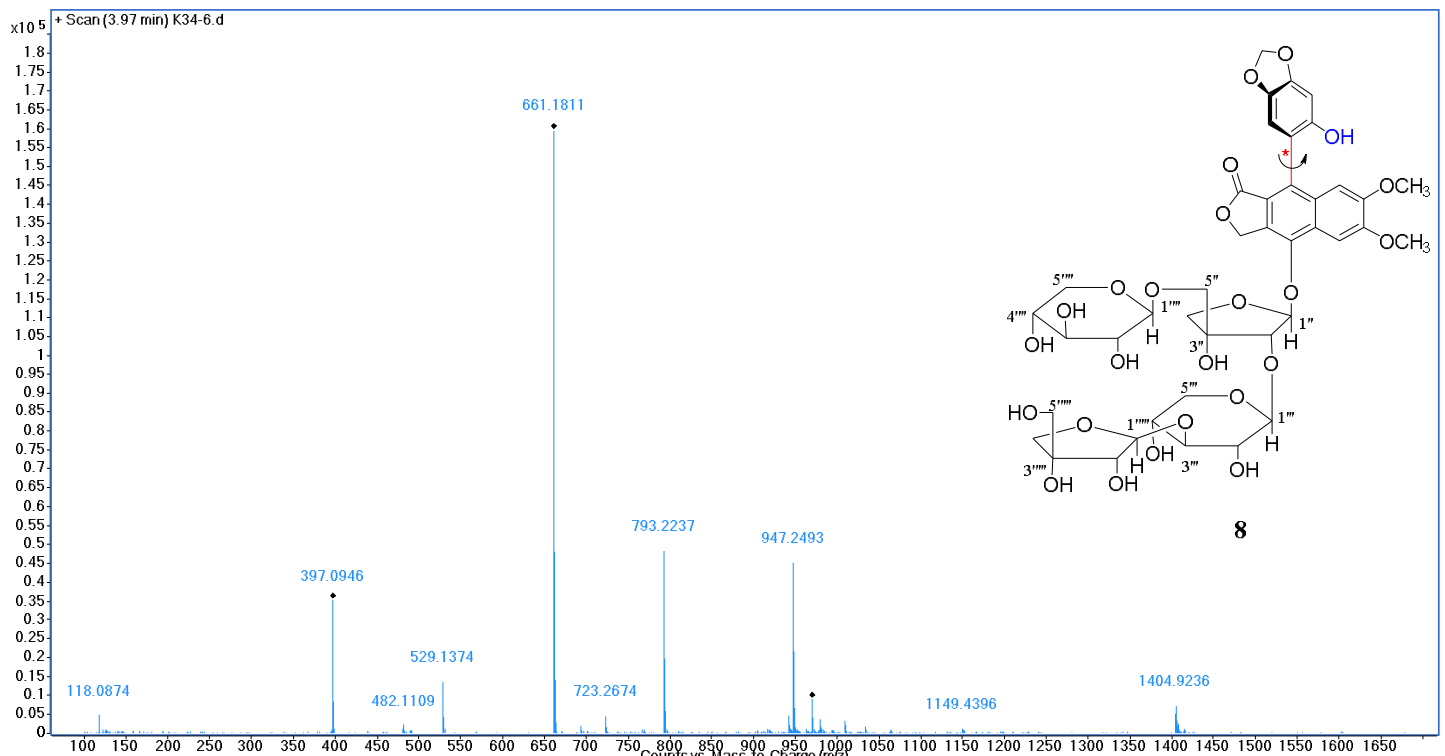

Figure S69. HRESIMS Data of Compound 8.

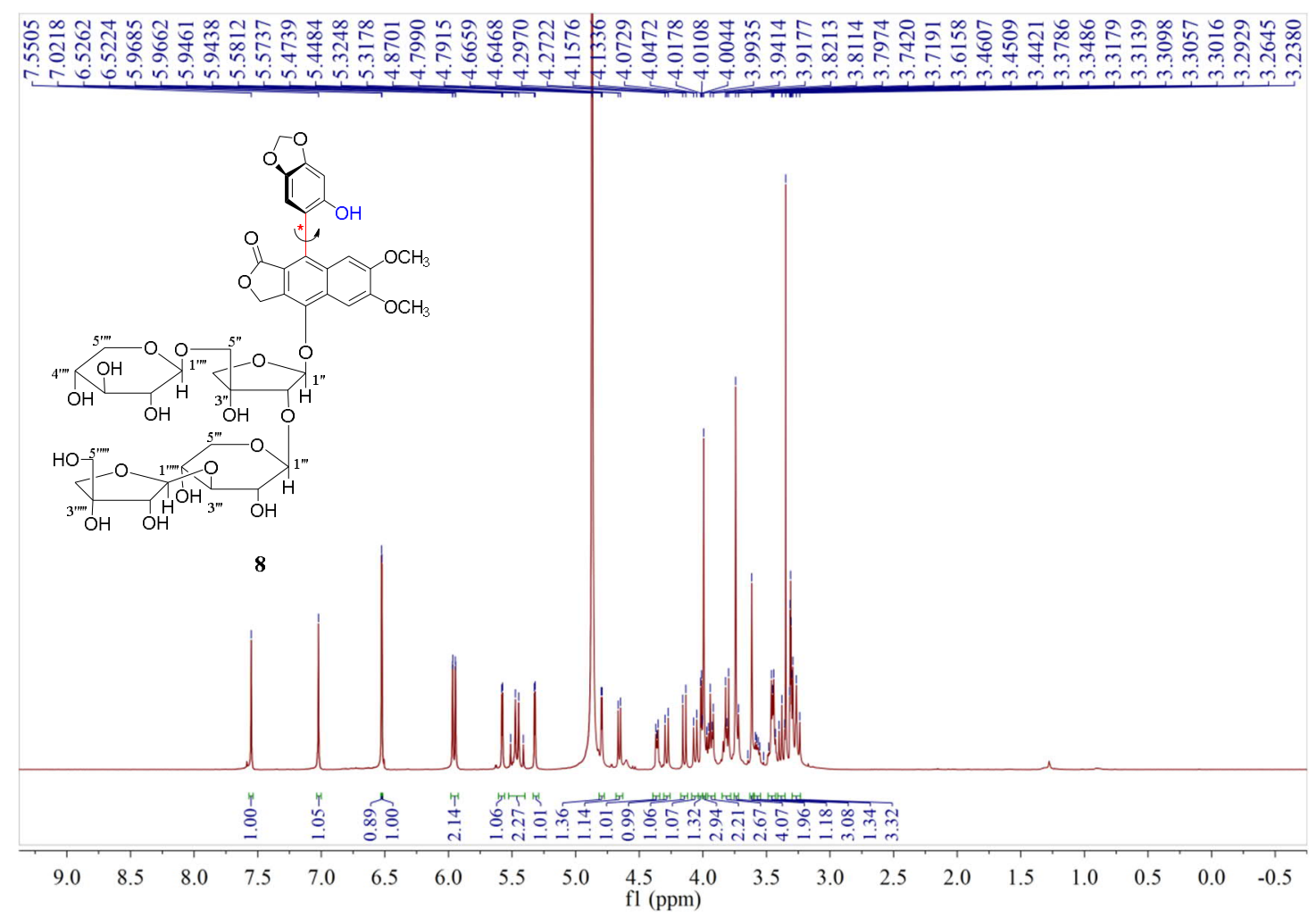

Figure S70. The ${ }^{1} \mathrm{H}$ NMR Spectrum of Compound 8 in $\mathrm{CD}_{3} \mathrm{OD}(400 \mathrm{MHz})$. 


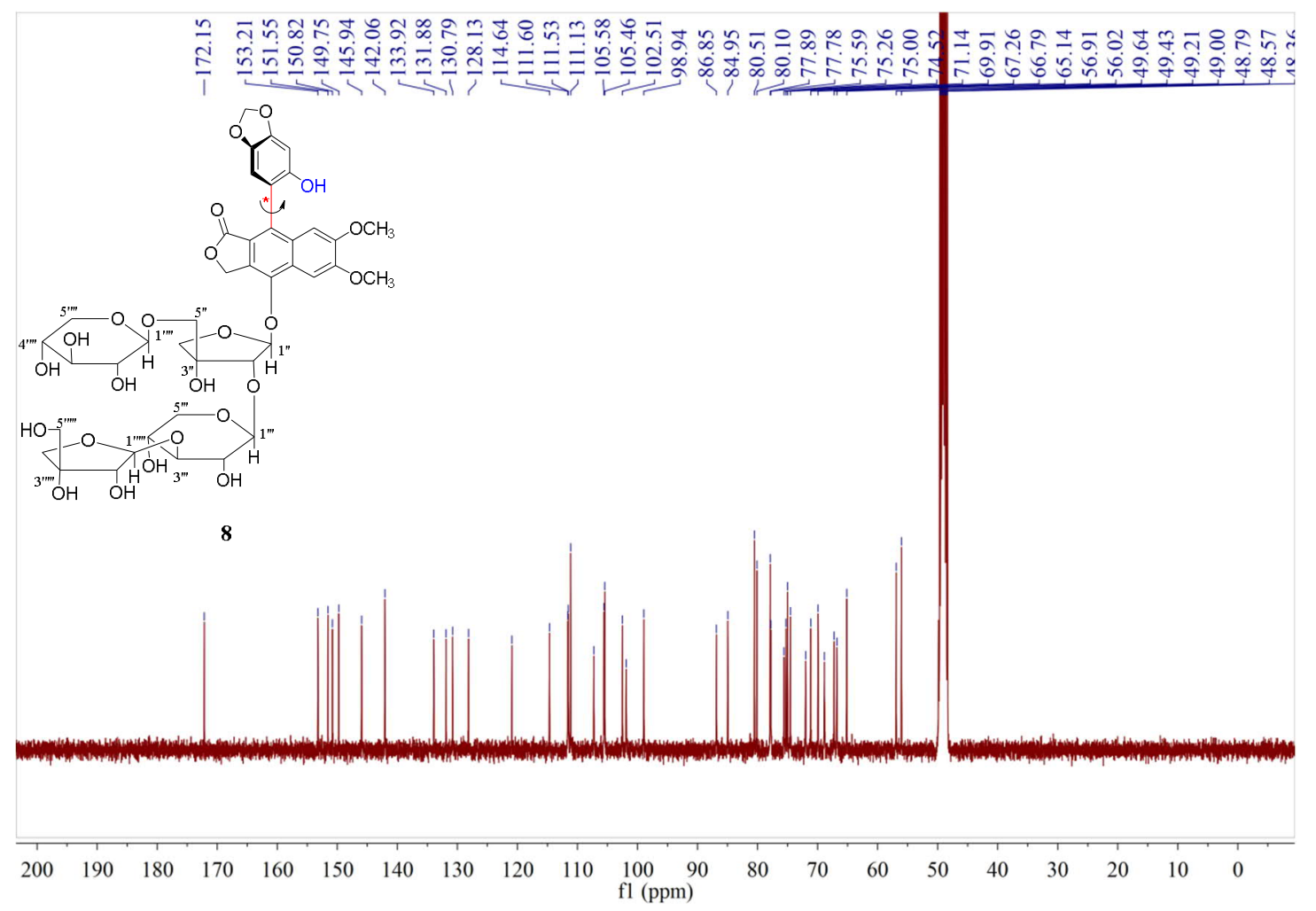

Figure S71. The ${ }^{13} \mathrm{C}\left\{{ }^{1} \mathrm{H}\right\}$ NMR Spectrum of Compound 8 in $\mathrm{CD}_{3} \mathrm{OD}(100 \mathrm{MHz})$.

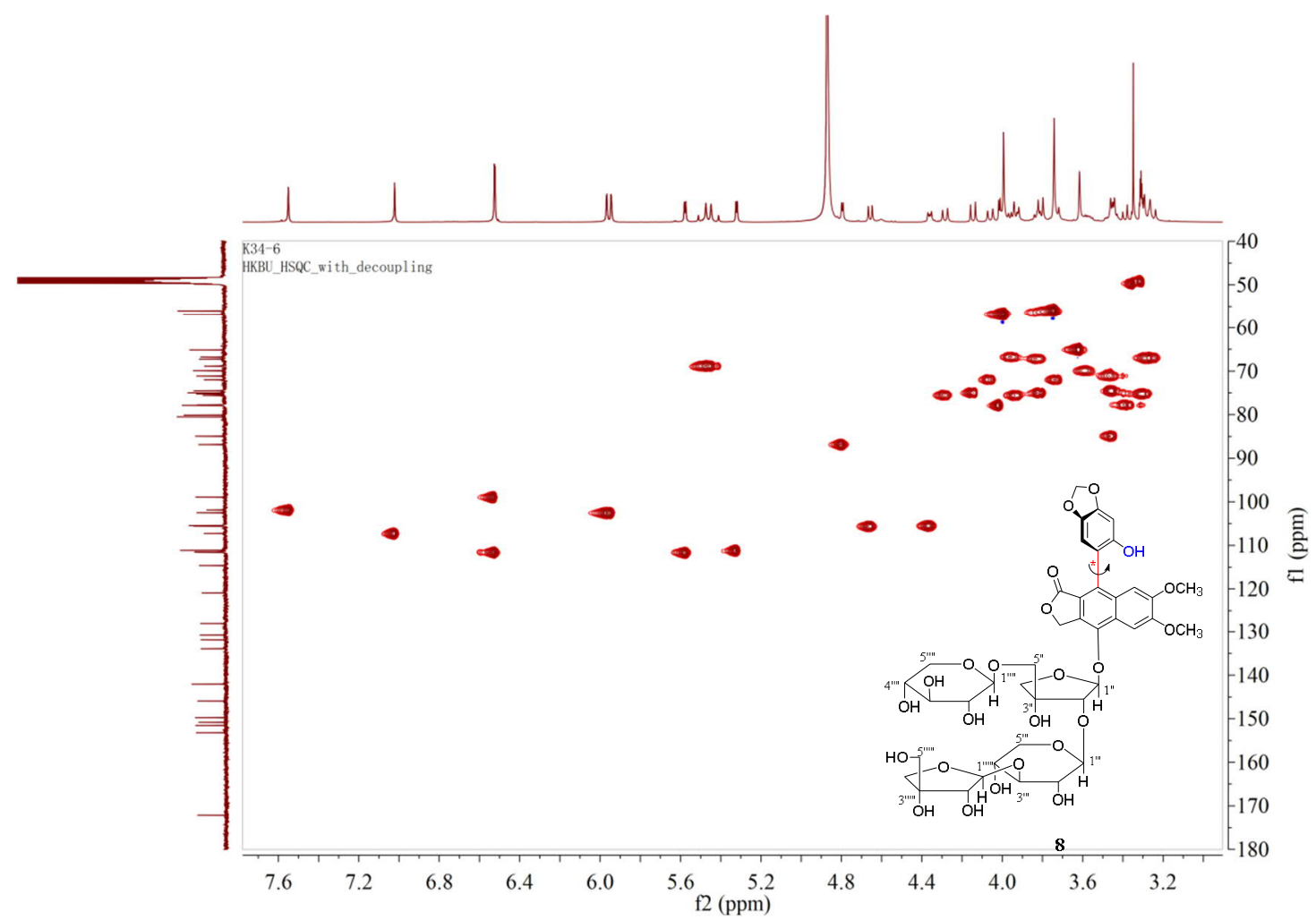

Figure S72. The HSQC Spectrum of Compound 8 in $\mathrm{CD}_{3} \mathrm{OD}\left(400 \mathrm{MHz}\right.$ for $\left.{ }^{1} \mathrm{H}\right)$. 


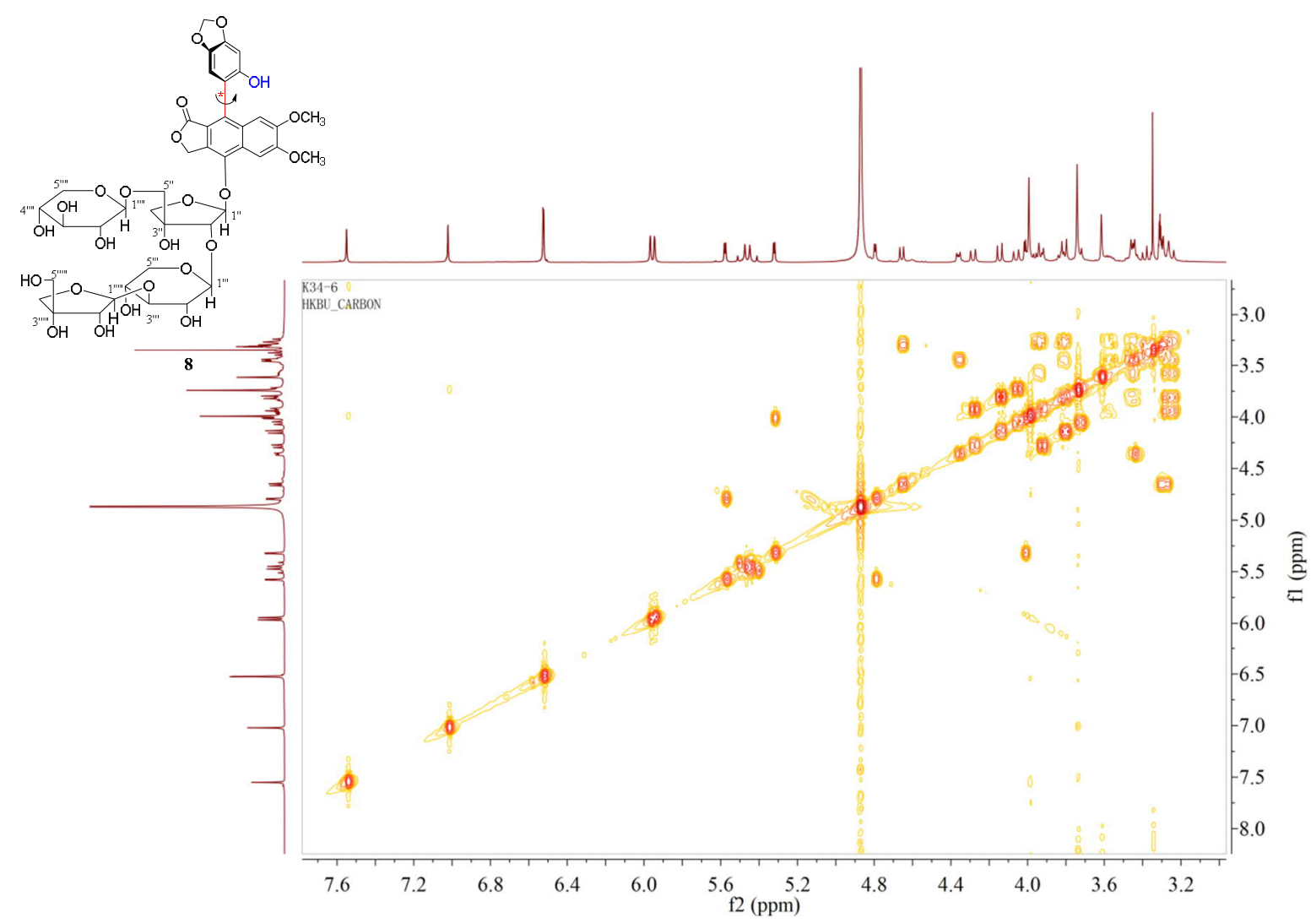

Figure S73. The ${ }^{1} \mathrm{H}-{ }^{1} \mathrm{H}$ COSY Spectrum of Compound 8 in $\mathrm{CD}_{3} \mathrm{OD}(400 \mathrm{MHz})$.

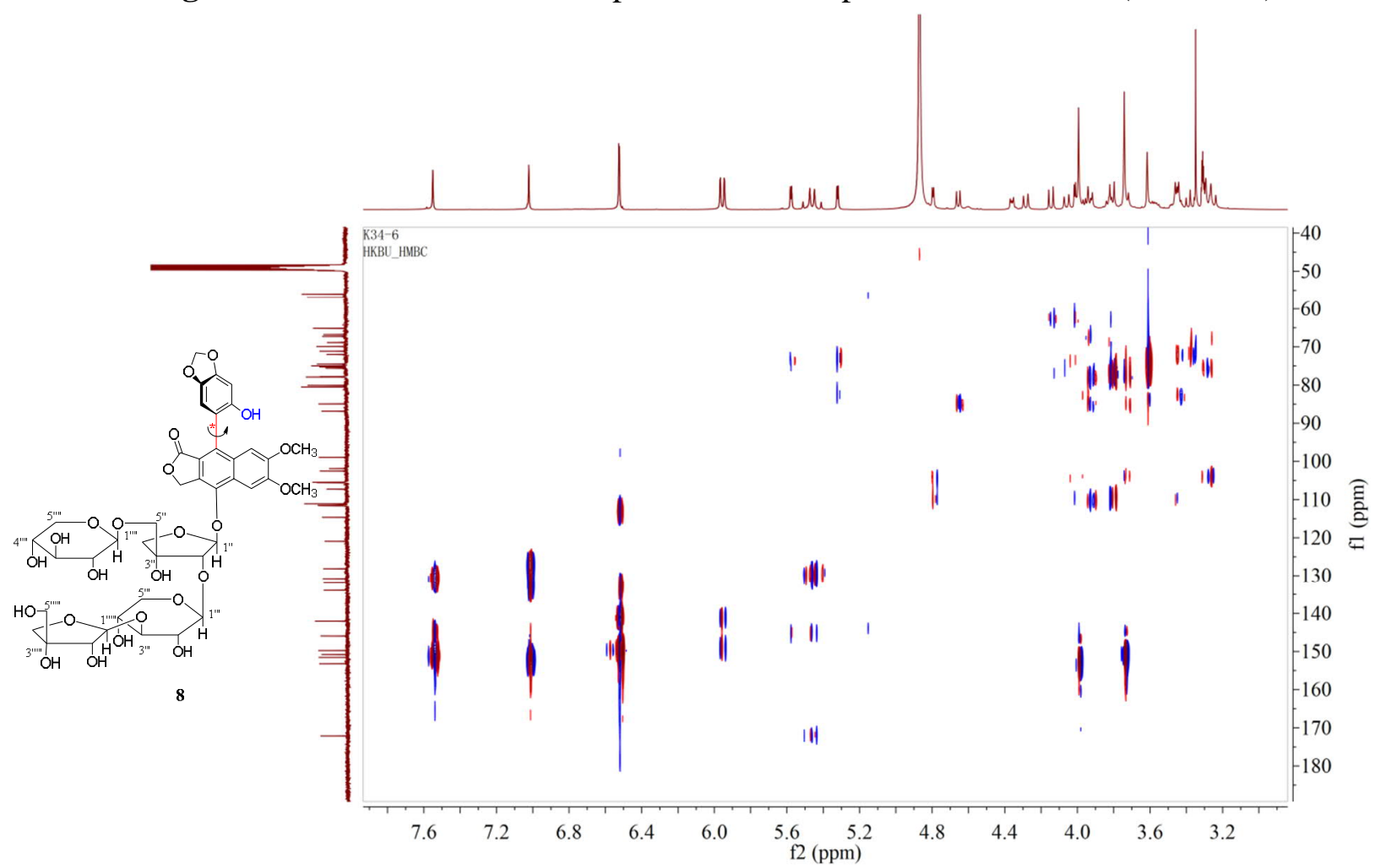

Figure S74. The HMBC Spectrum of Compound 8 in $\mathrm{CD}_{3} \mathrm{OD}\left(400 \mathrm{MHz}\right.$ for $\left.{ }^{1} \mathrm{H}\right)$. 


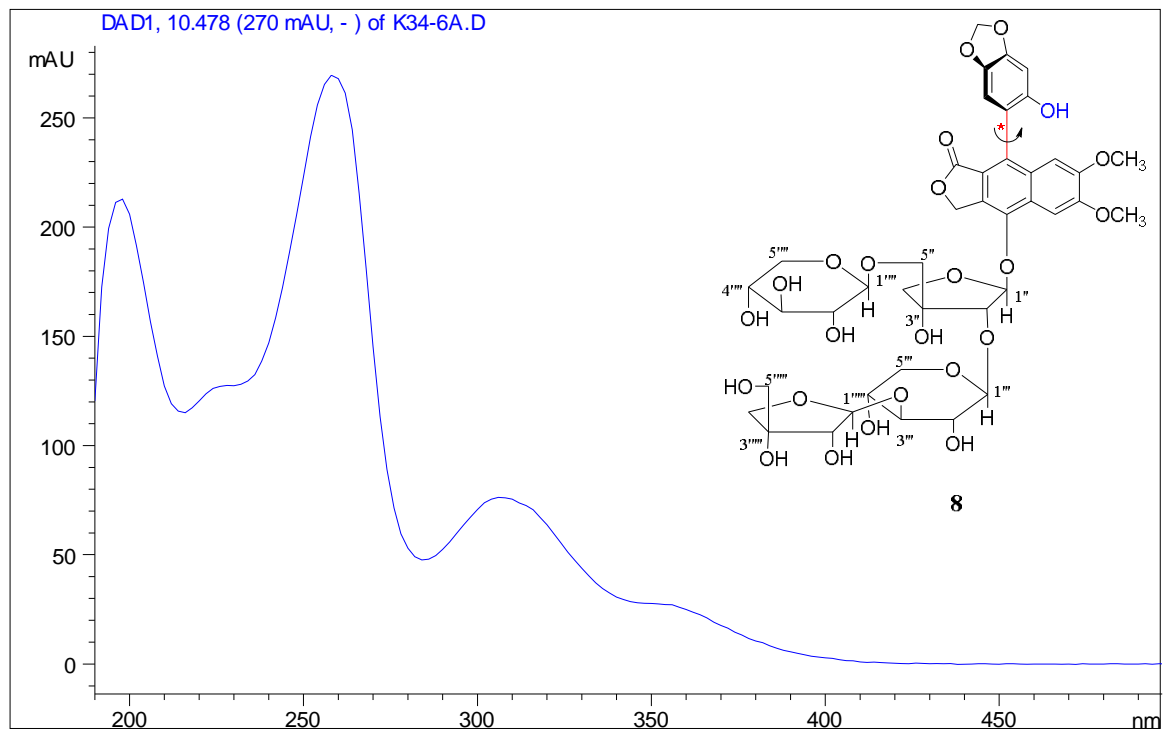

Figure S75. The UV Spectrum of Compound 8.

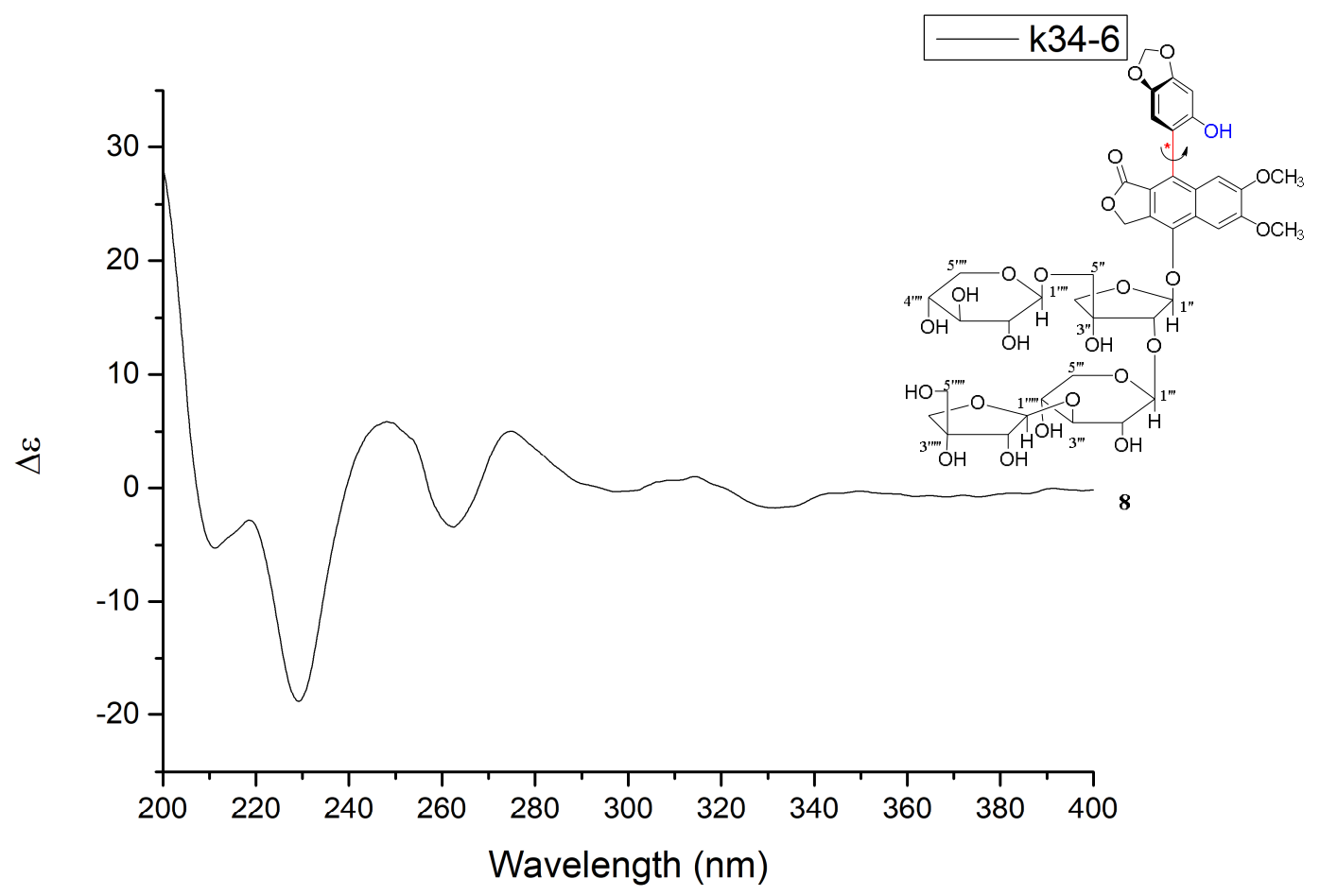

Figure S76. The CD Spectrum of Compound 8. 


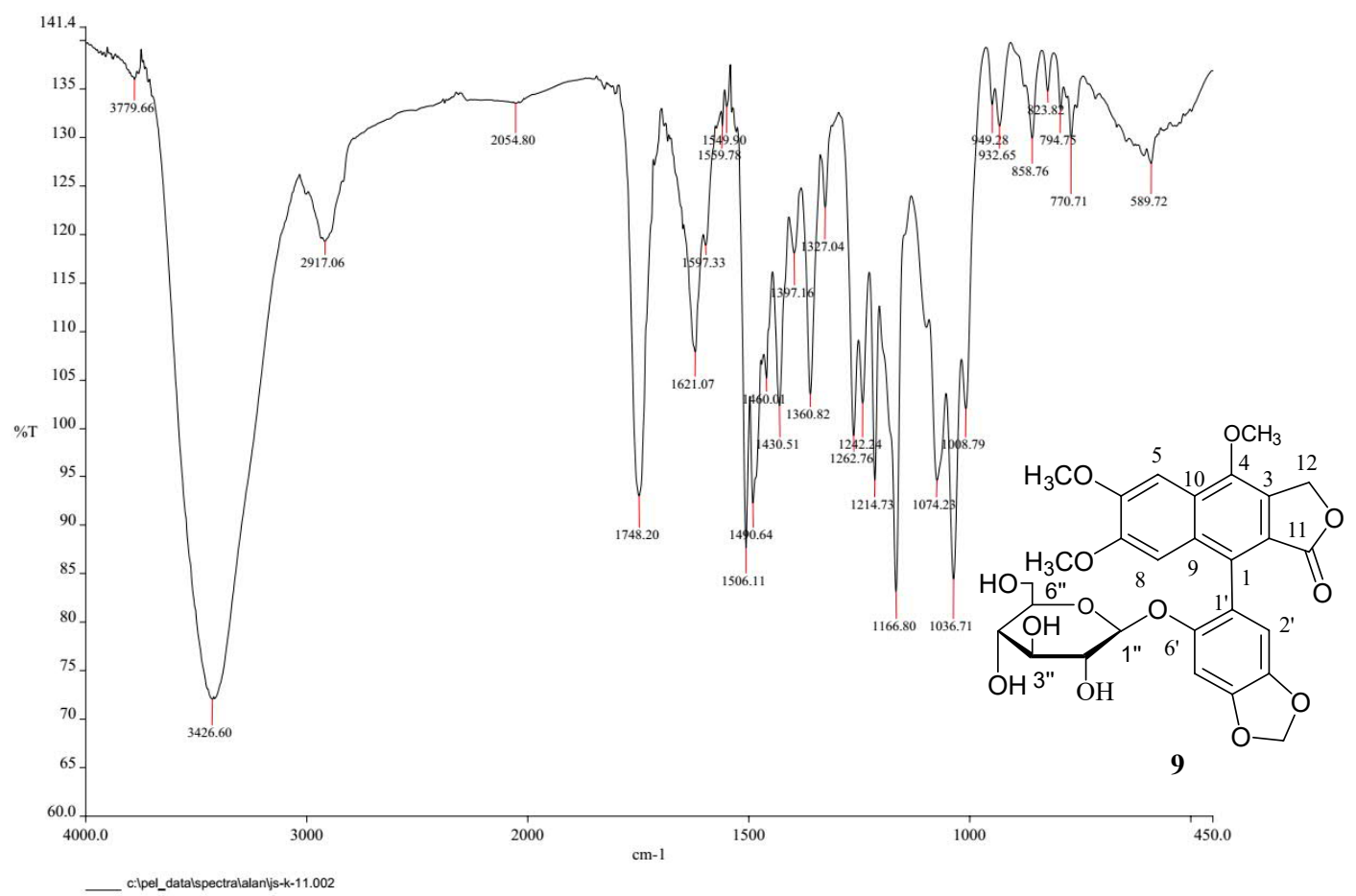

Figure S77. The IR Spectrum of Compound 9.

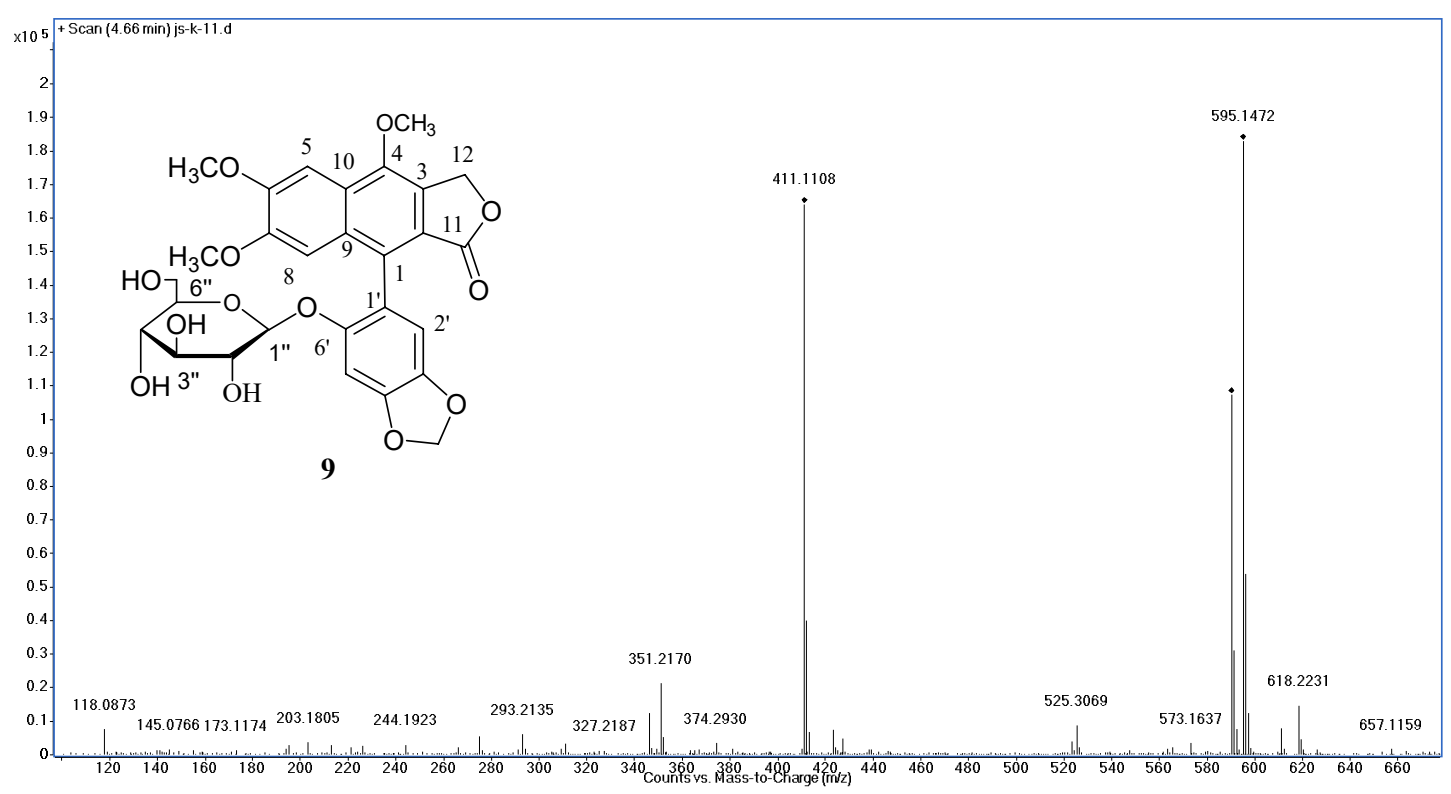

Figure S78. HRESIMS Data of Compound 9. 


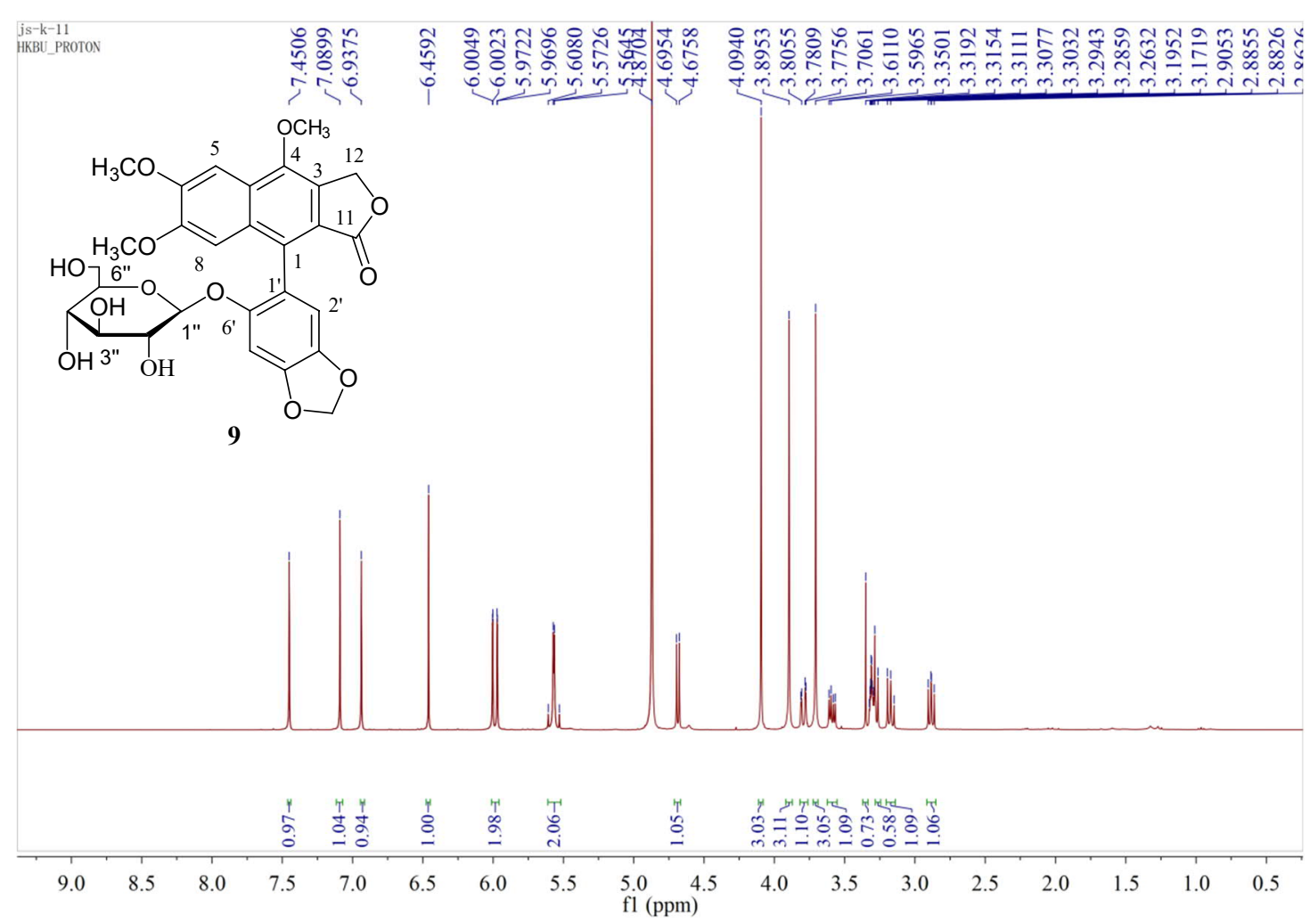

Figure S79. The ${ }^{1} \mathrm{H}$ NMR Spectrum of Compound 9 in $\mathrm{CD}_{3} \mathrm{OD}(400 \mathrm{MHz})$.

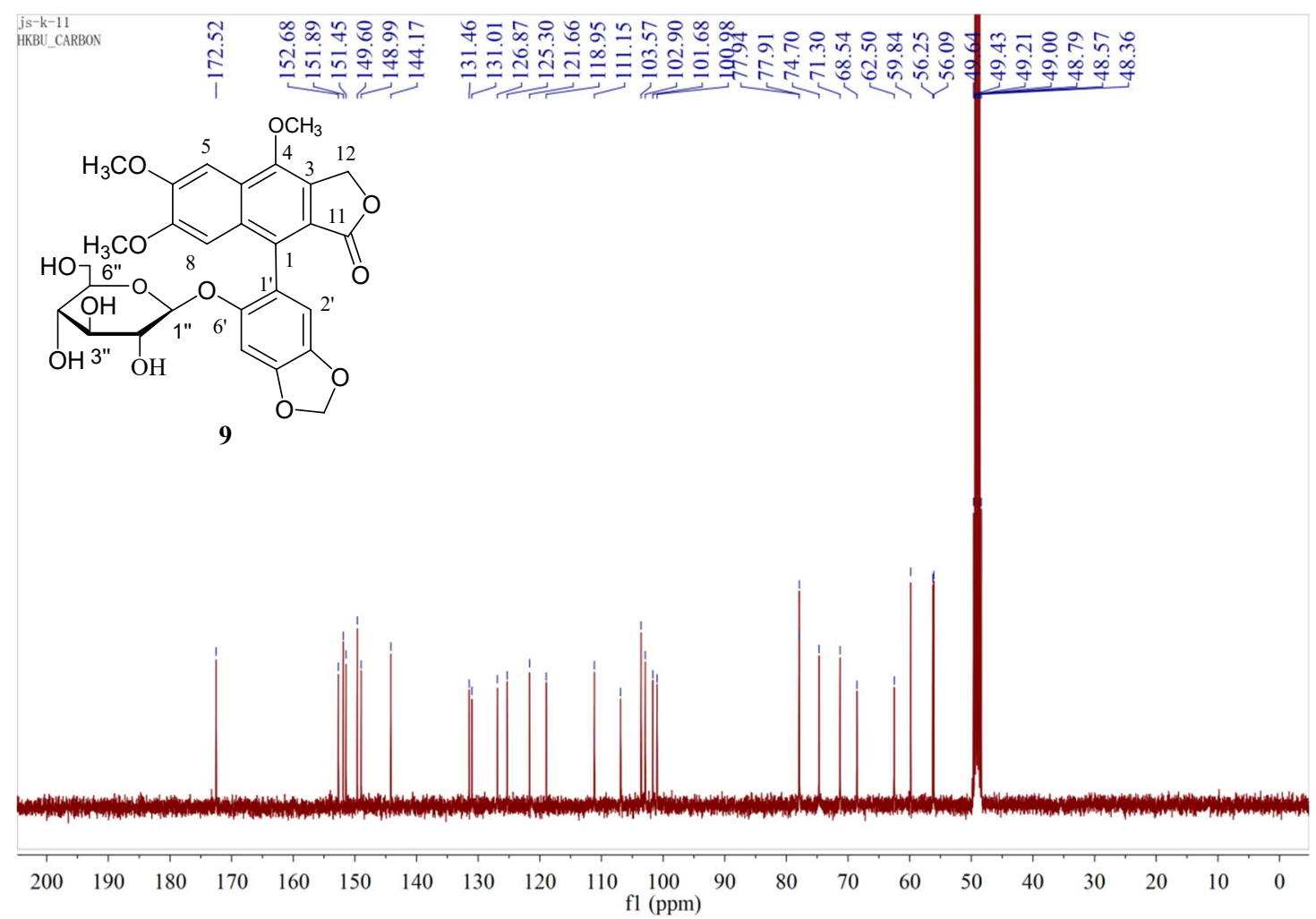

Figure S80. The ${ }^{13} \mathrm{C}\left\{{ }^{1} \mathrm{H}\right\}$ NMR Spectrum of Compound 9 in $\mathrm{CD}_{3} \mathrm{OD}(100 \mathrm{MHz})$. 


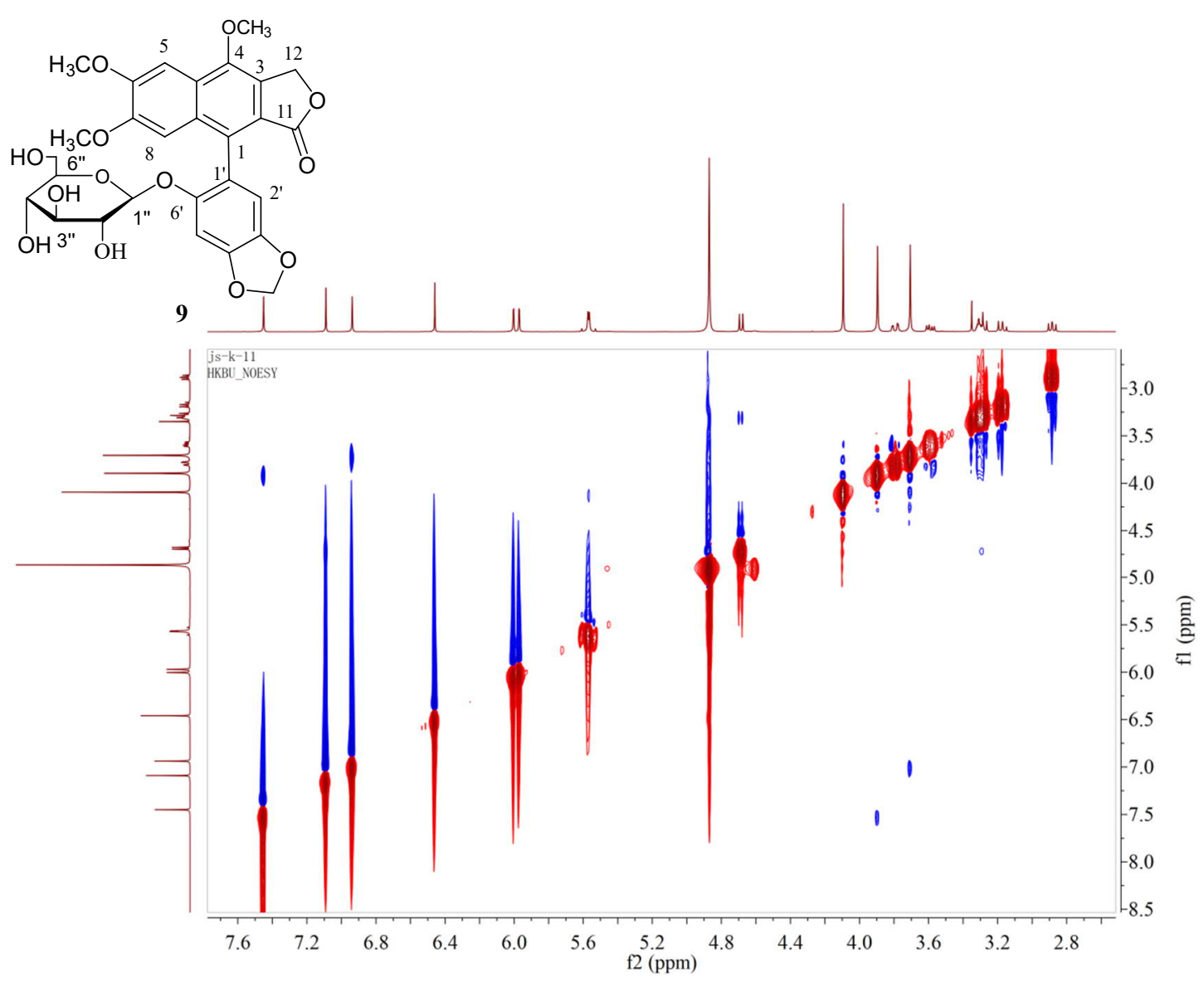

Figure S81. The NOESY Spectrum of Compound 9 in $\mathrm{CD}_{3} \mathrm{OD}(400 \mathrm{MHz})$.

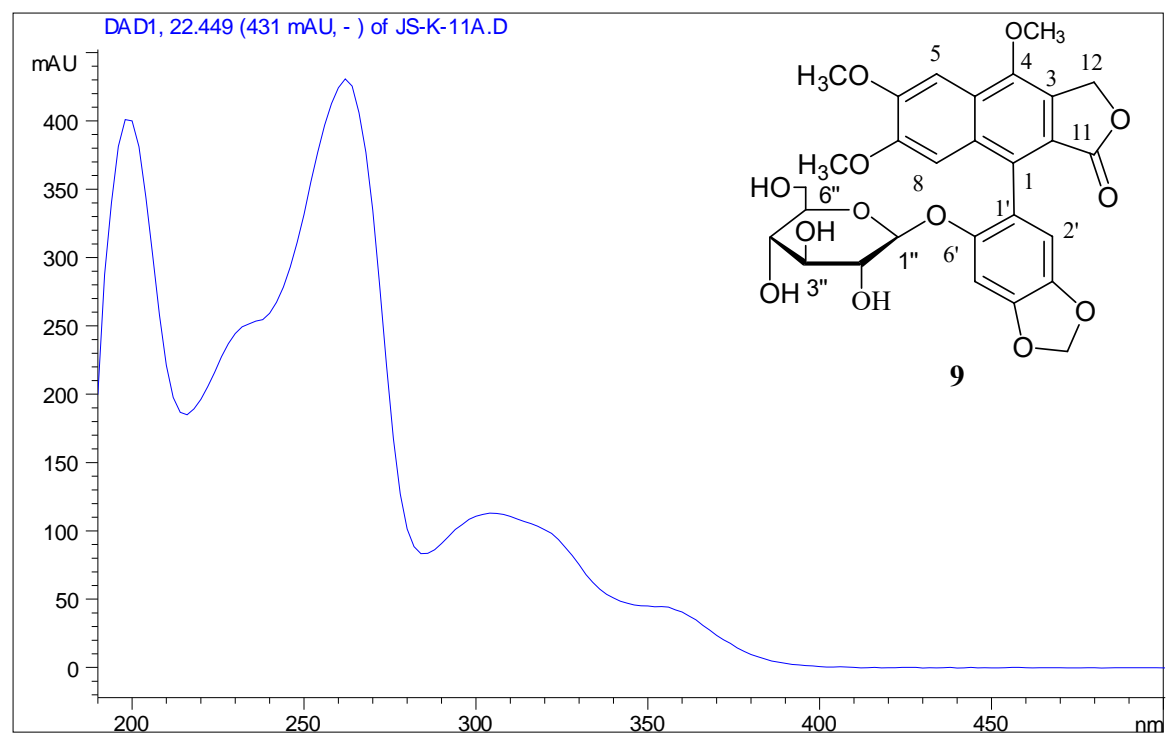

Figure S82. The UV Spectrum of Compound 9. 


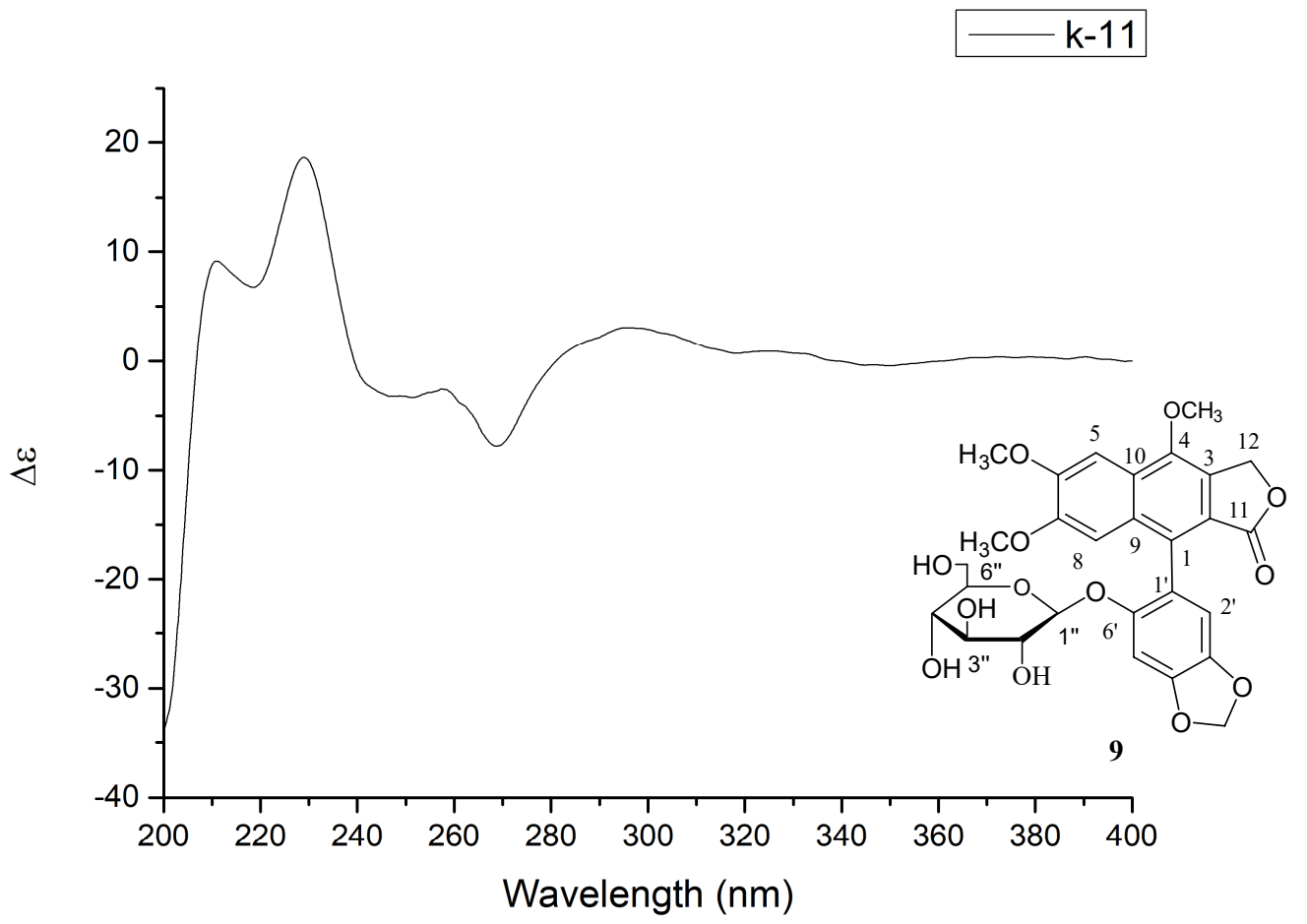

Figure S83. The CD Spectrum of Compound 9.

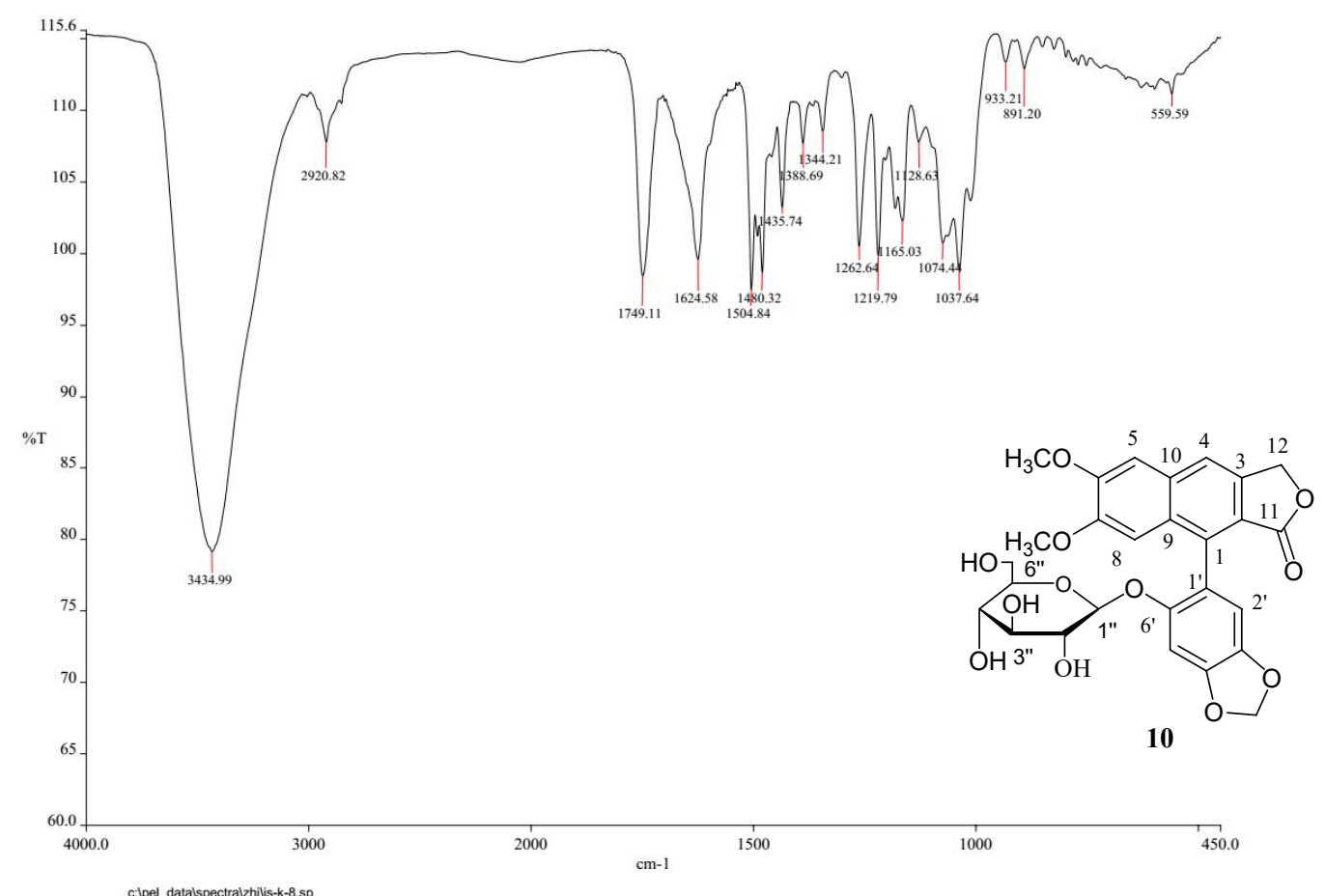

Figure S84. The IR Spectrum of Compound 10. 


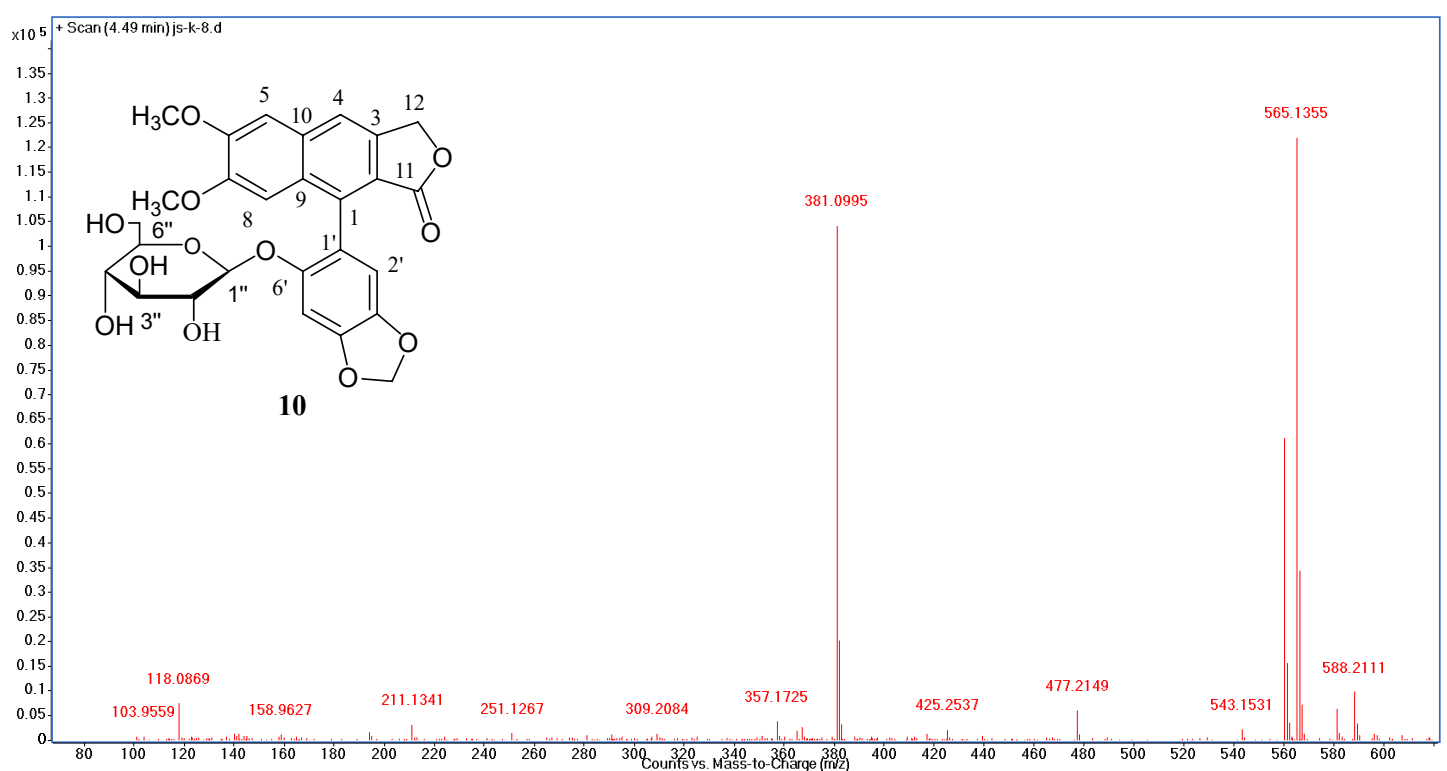

Figure S85. HRESIMS Data of Compound 10.

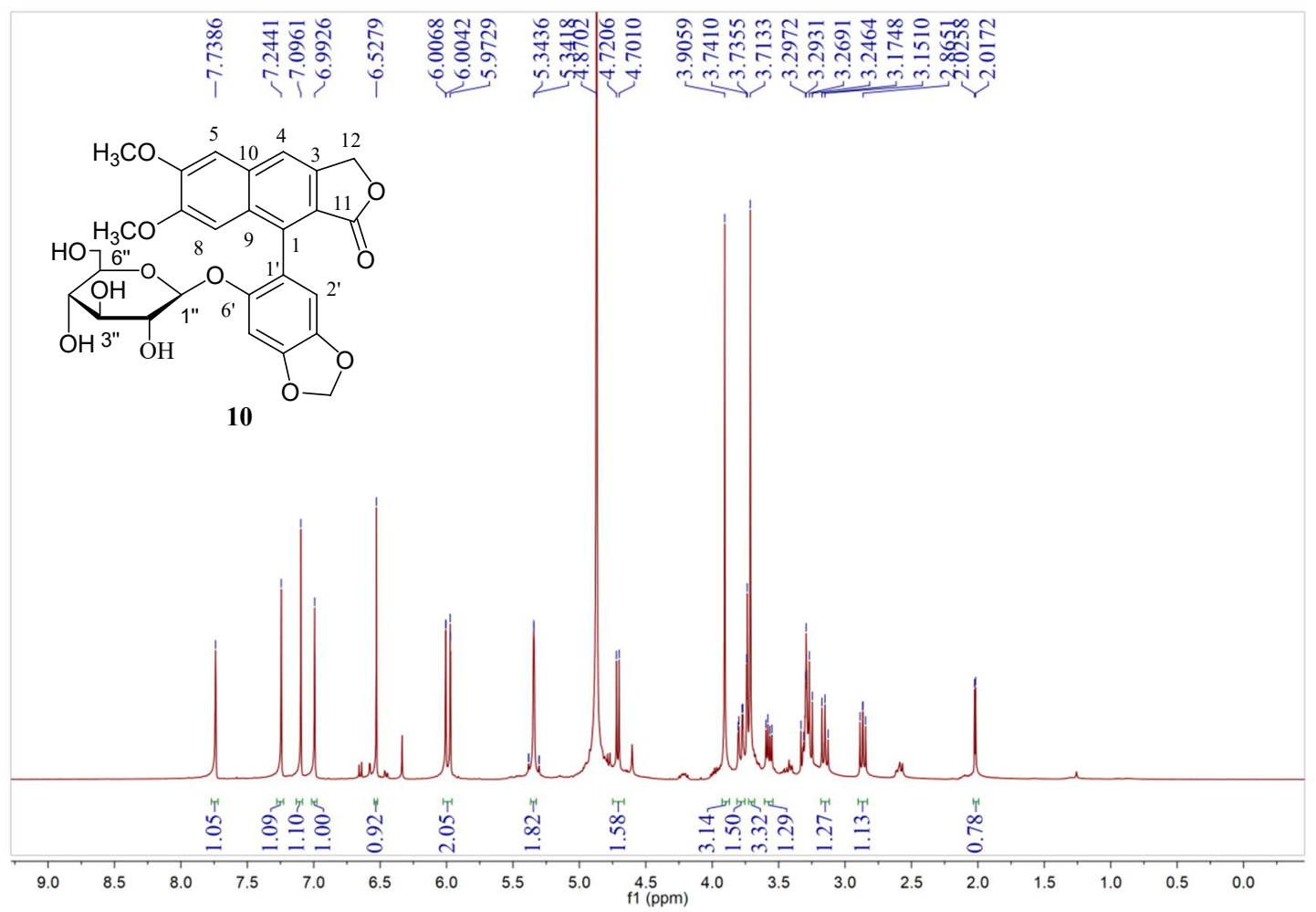

Figure S86. The ${ }^{1} \mathrm{H}$ NMR Spectrum of Compound 10 in $\mathrm{CD}_{3} \mathrm{OD}(400 \mathrm{MHz})$. 


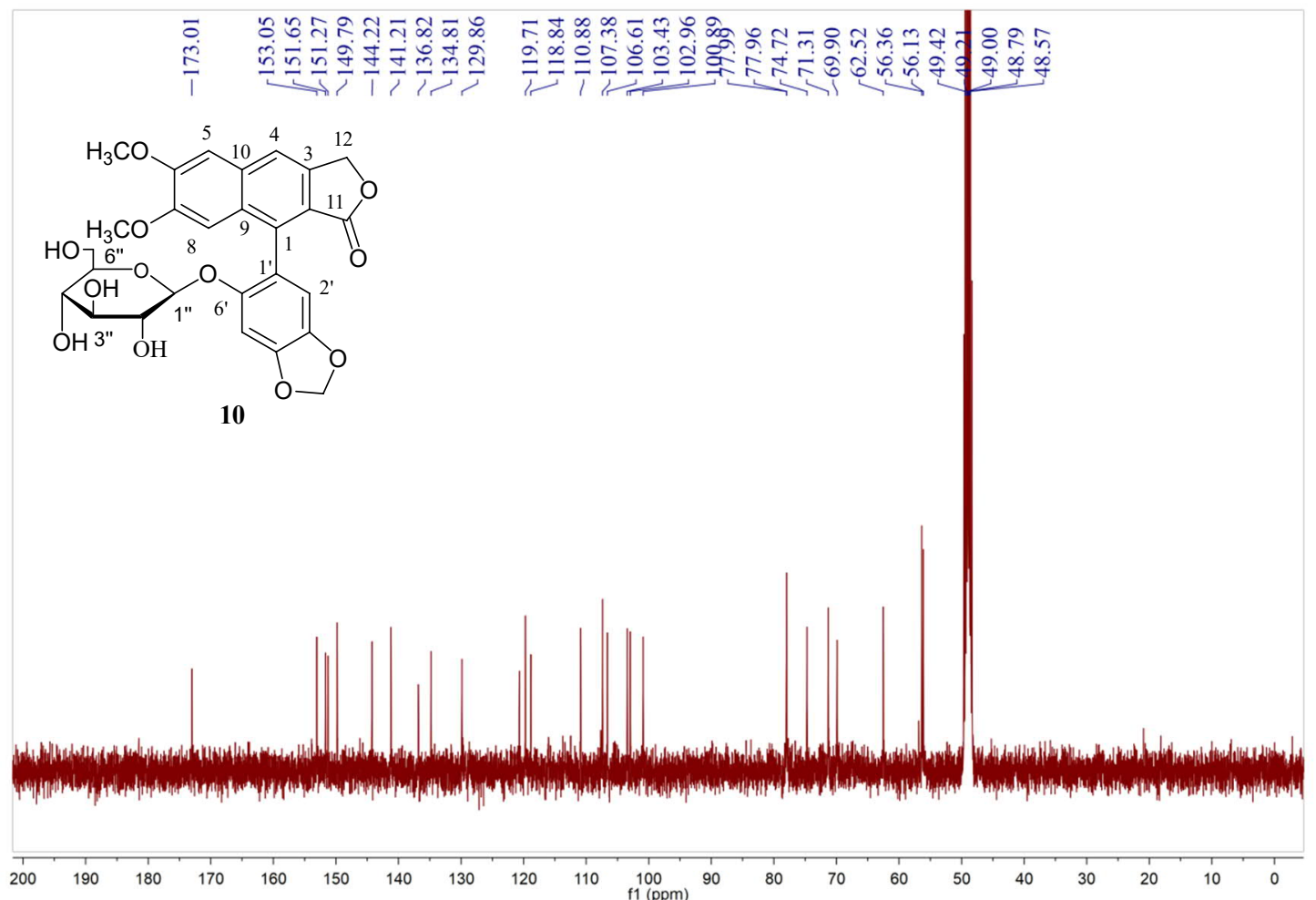

Figure S87. The ${ }^{13} \mathrm{C}\left\{{ }^{1} \mathrm{H}\right\}$ NMR Spectrum of Compound 10 in $\mathrm{CD}_{3} \mathrm{OD}(100 \mathrm{MHz})$.

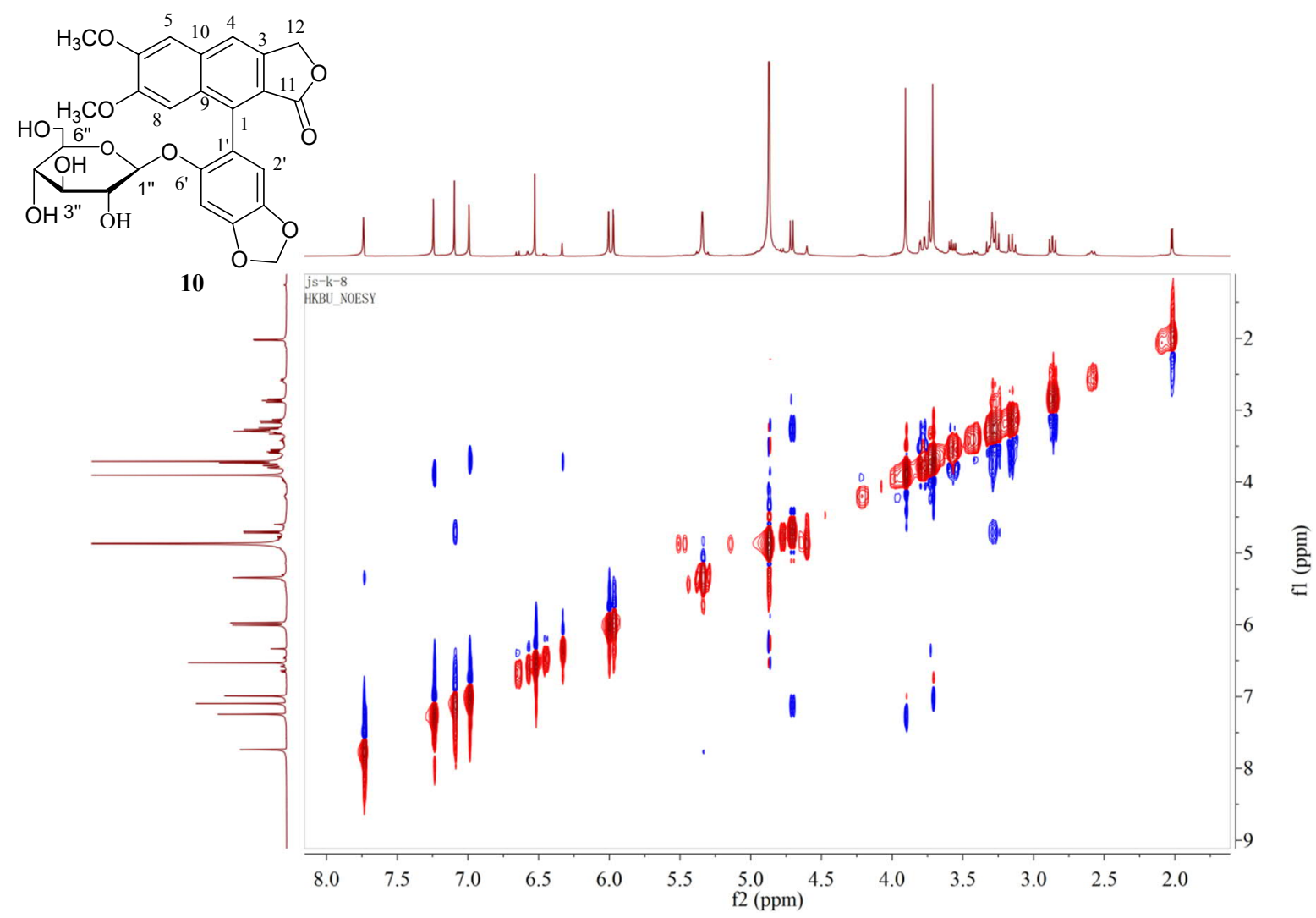

Figure S88. The NOESY Spectrum of Compound 10 in $\mathrm{CD}_{3} \mathrm{OD}(400 \mathrm{MHz})$. 


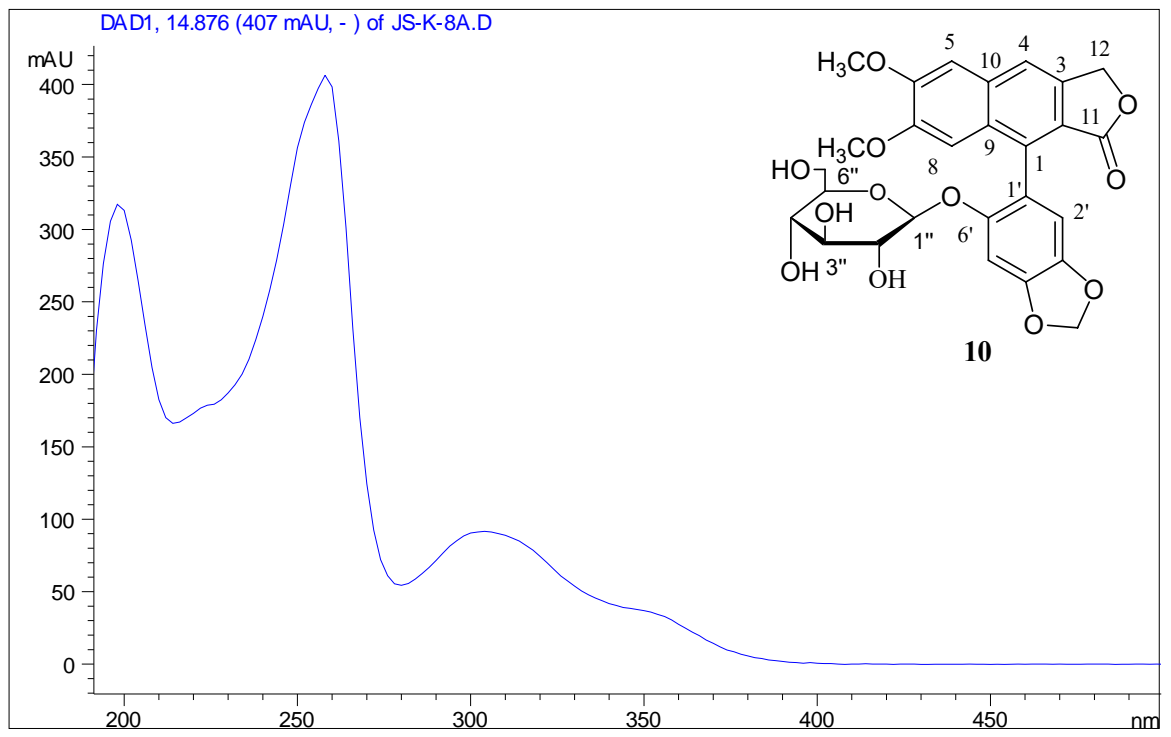

Figure S89. The UV Spectrum of Compound 10.

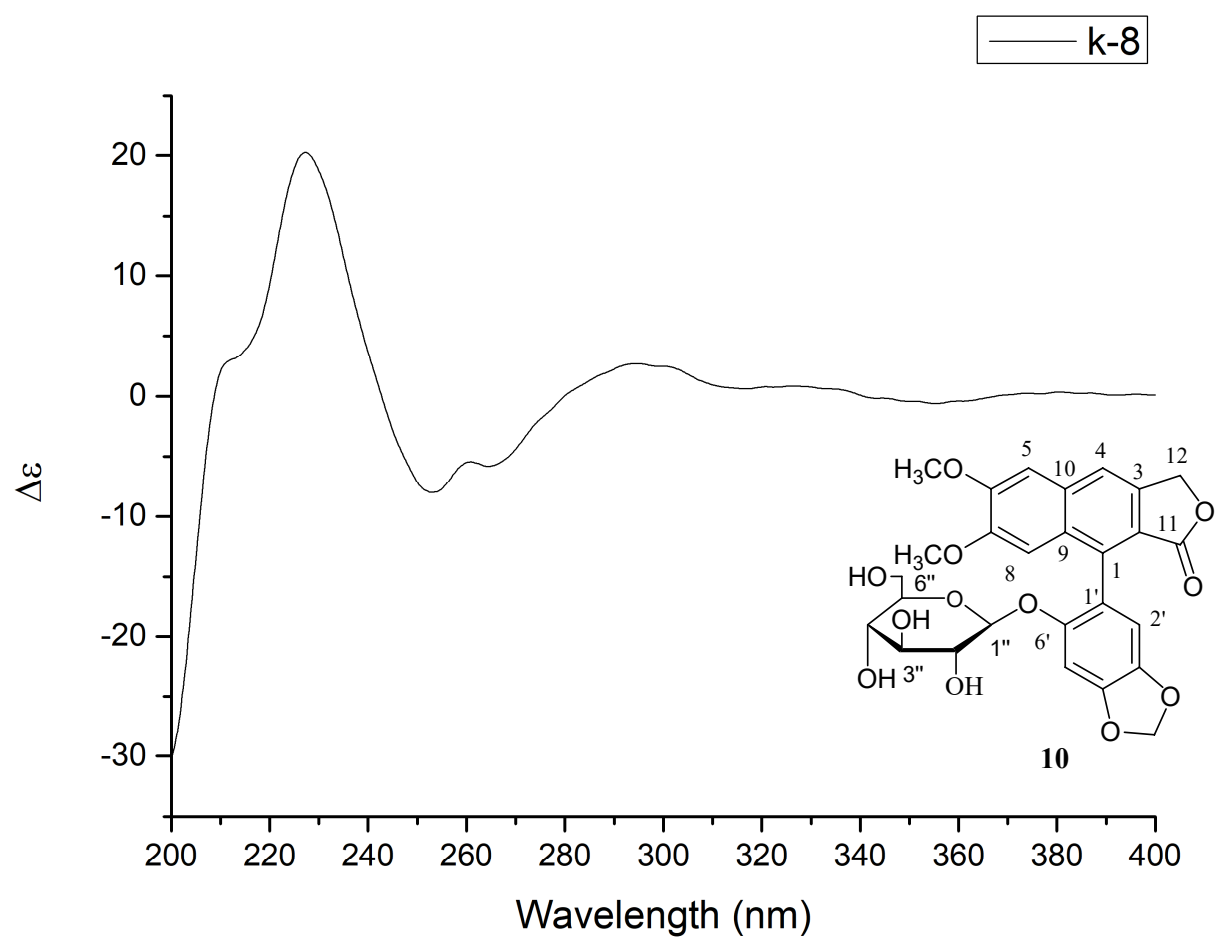

Figure S90. The CD Spectrum of Compound 10. 


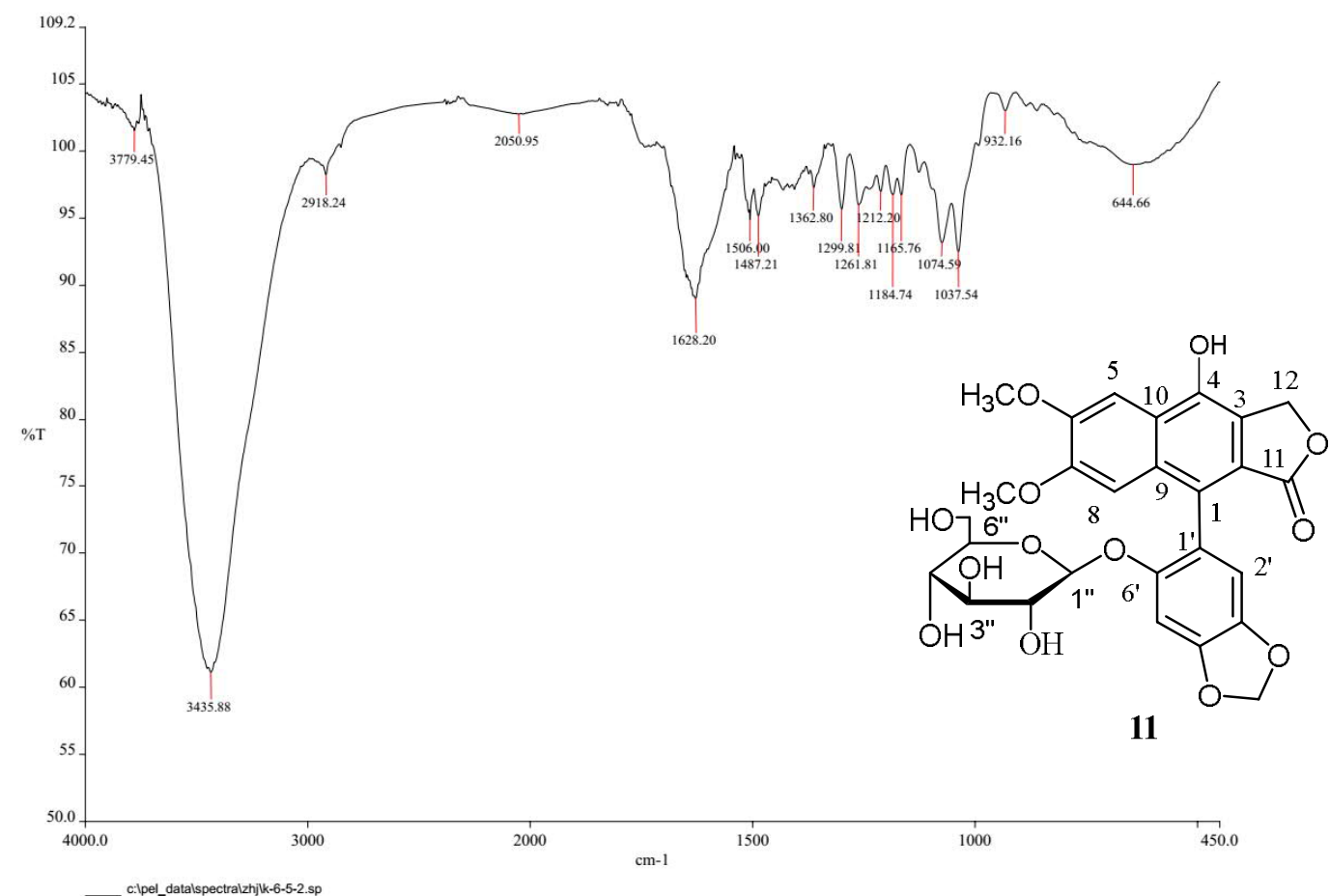

Figure S91. The IR Spectrum of Compound 11.

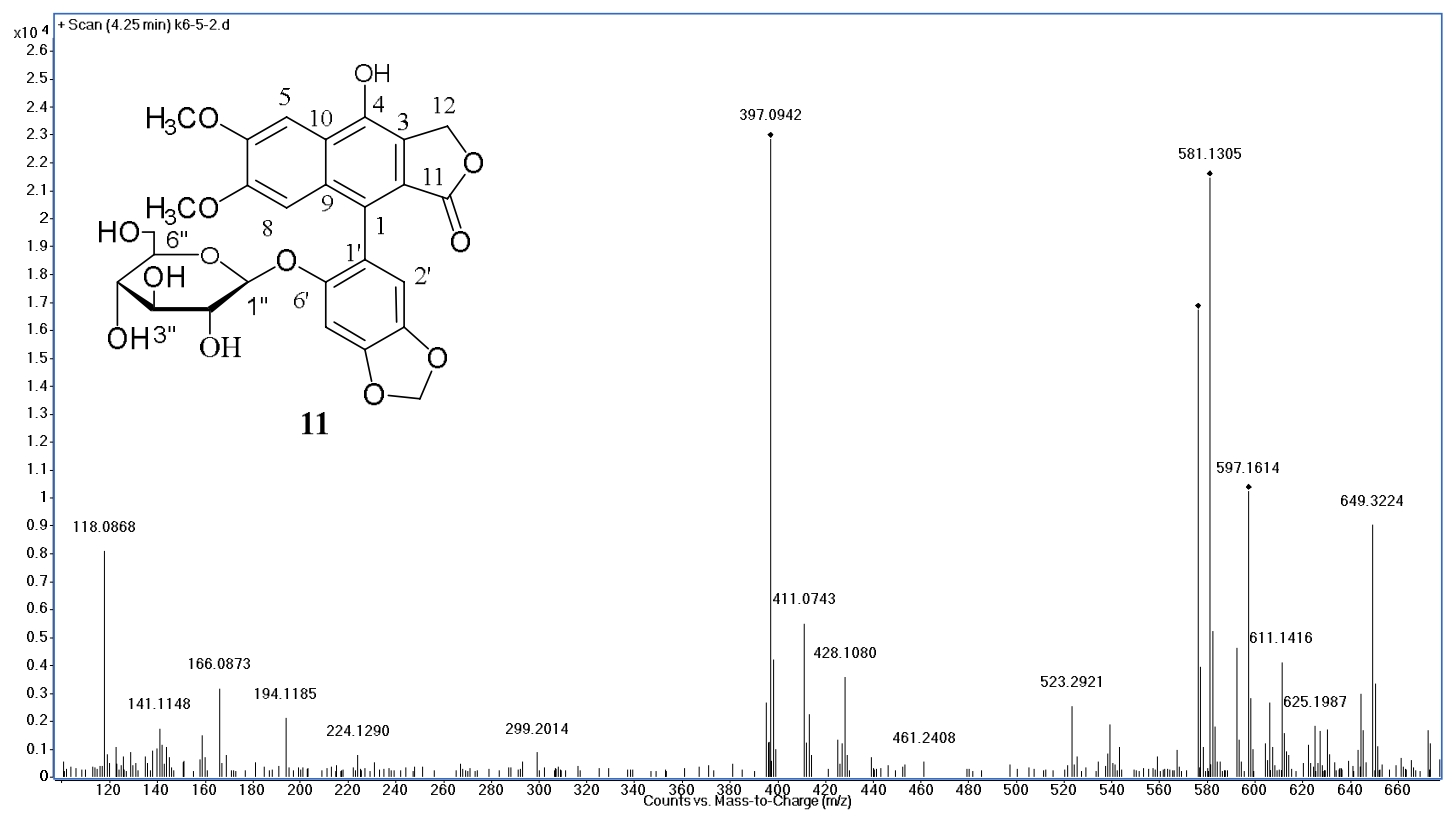

Figure S92. HRESIMS Data of Compound 11. 


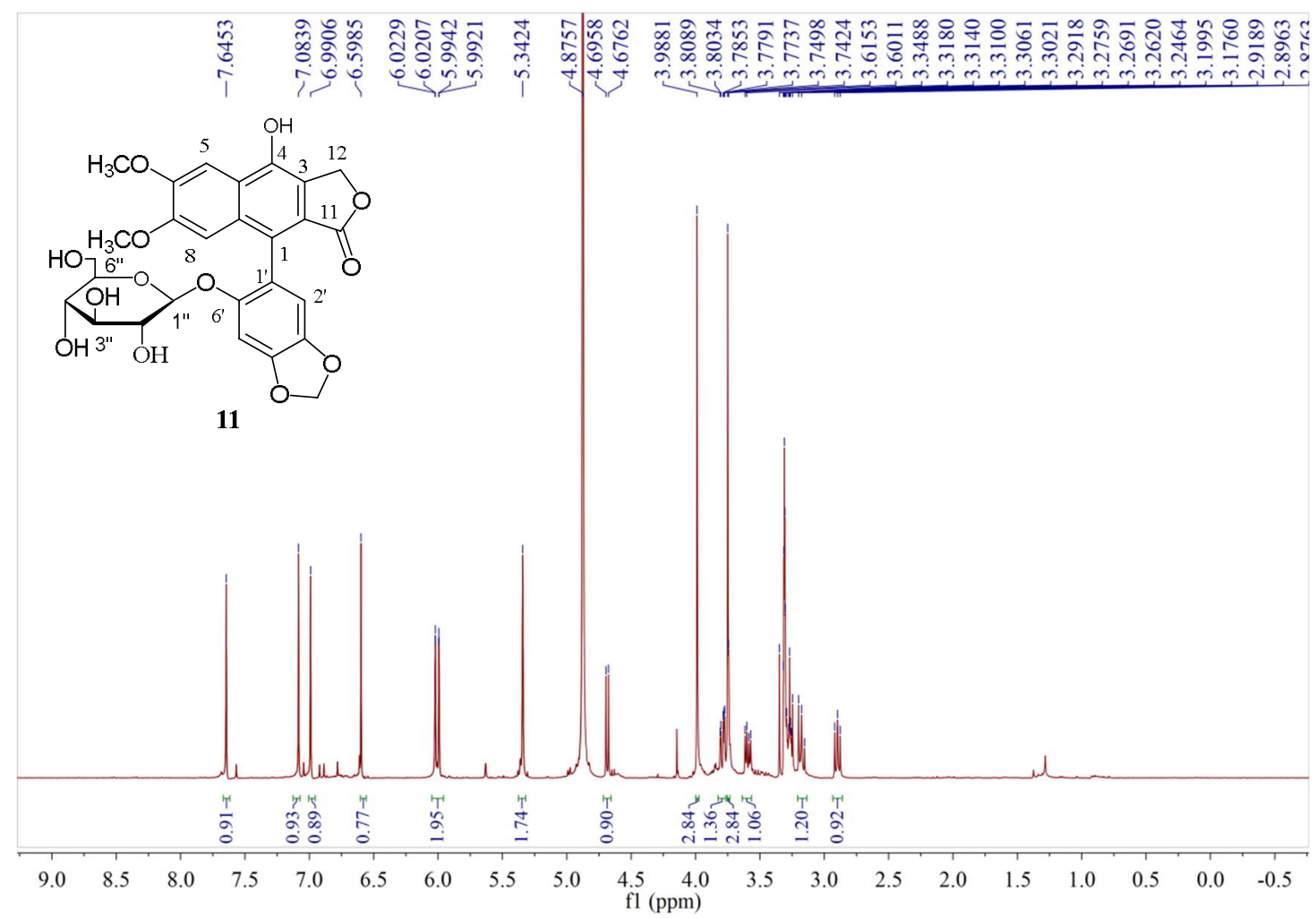

Figure S93. The ${ }^{1} \mathrm{H}$ NMR Spectrum of Compound 11 in $\mathrm{CD}_{3} \mathrm{OD}(400 \mathrm{MHz})$.

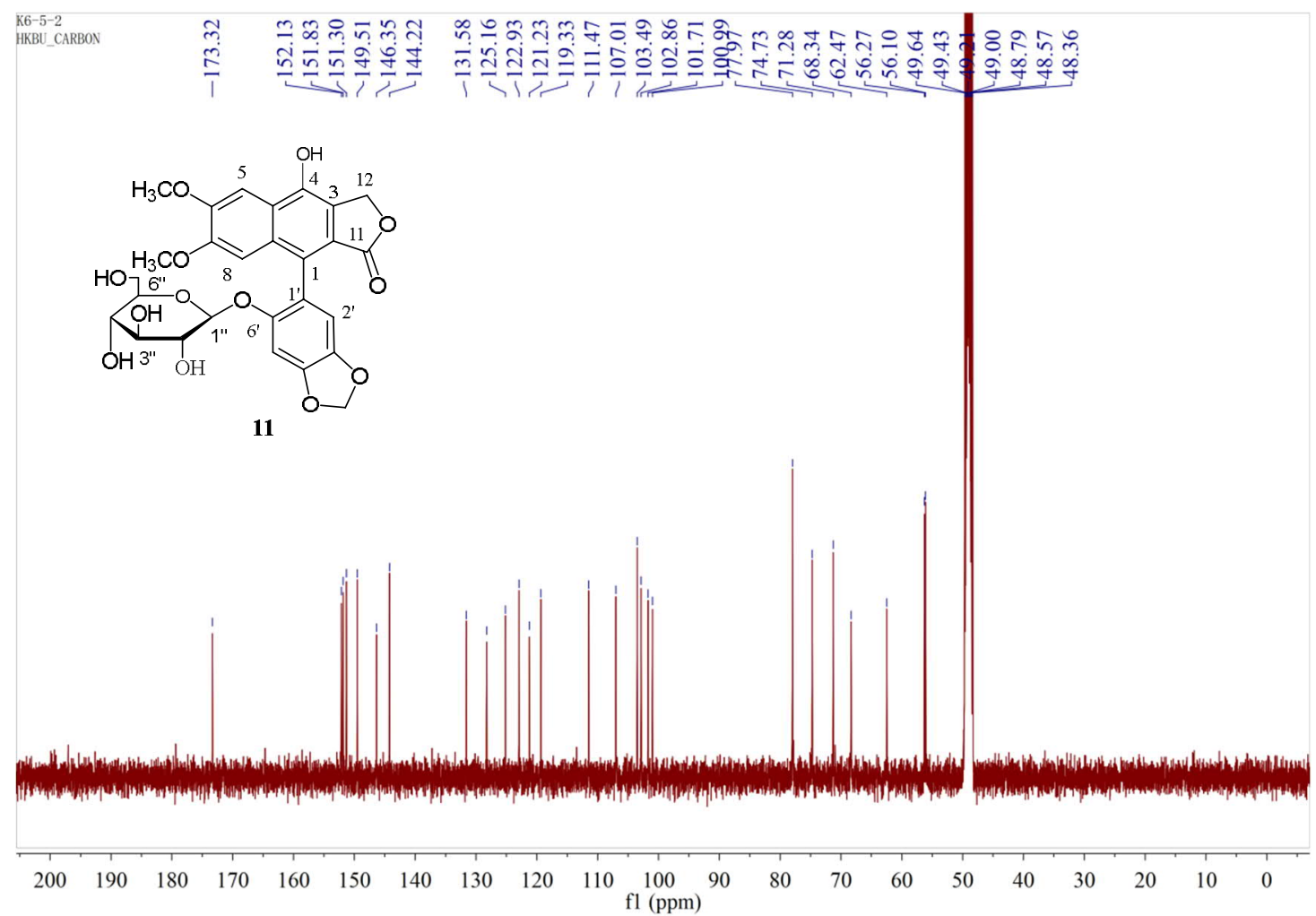

Figure S94. The ${ }^{13} \mathrm{C}\left\{{ }^{1} \mathrm{H}\right\}$ NMR Spectrum of Compound 11 in $\mathrm{CD}_{3} \mathrm{OD}(100 \mathrm{MHz})$. 


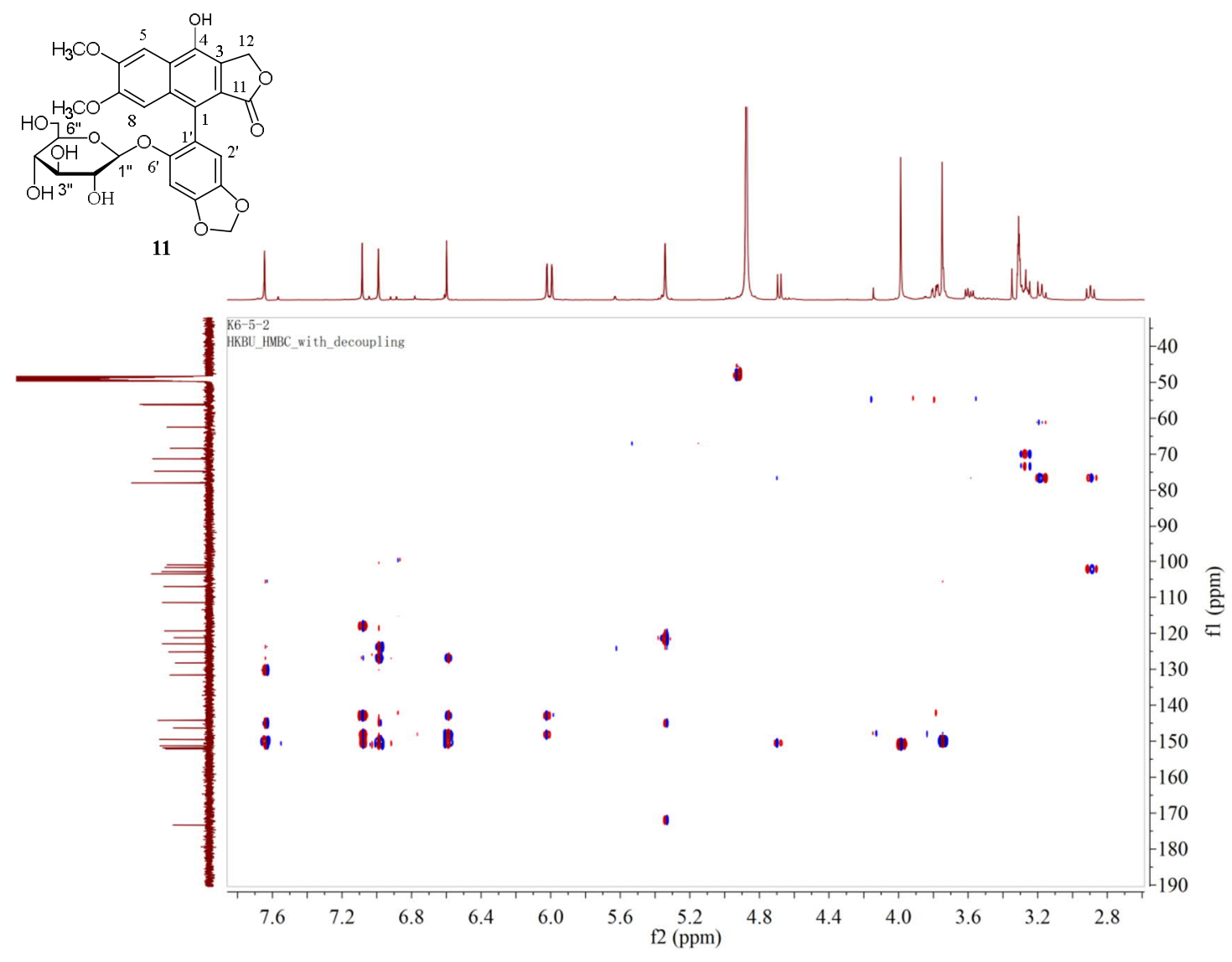

Figure S95. The HMBC Spectrum of Compound 11 in $\mathrm{CD}_{3} \mathrm{OD}\left(400 \mathrm{MHz}\right.$ for $\left.{ }^{1} \mathrm{H}\right)$.

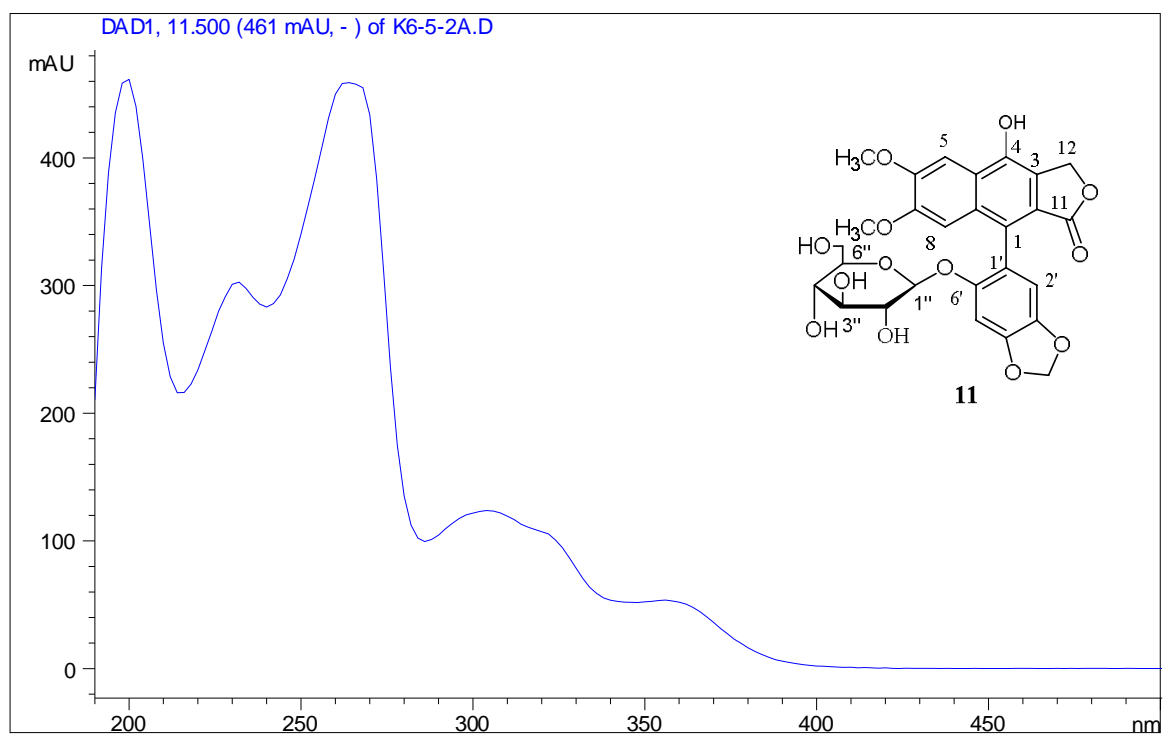

Figure S96. The UV Spectrum of Compound 11. 


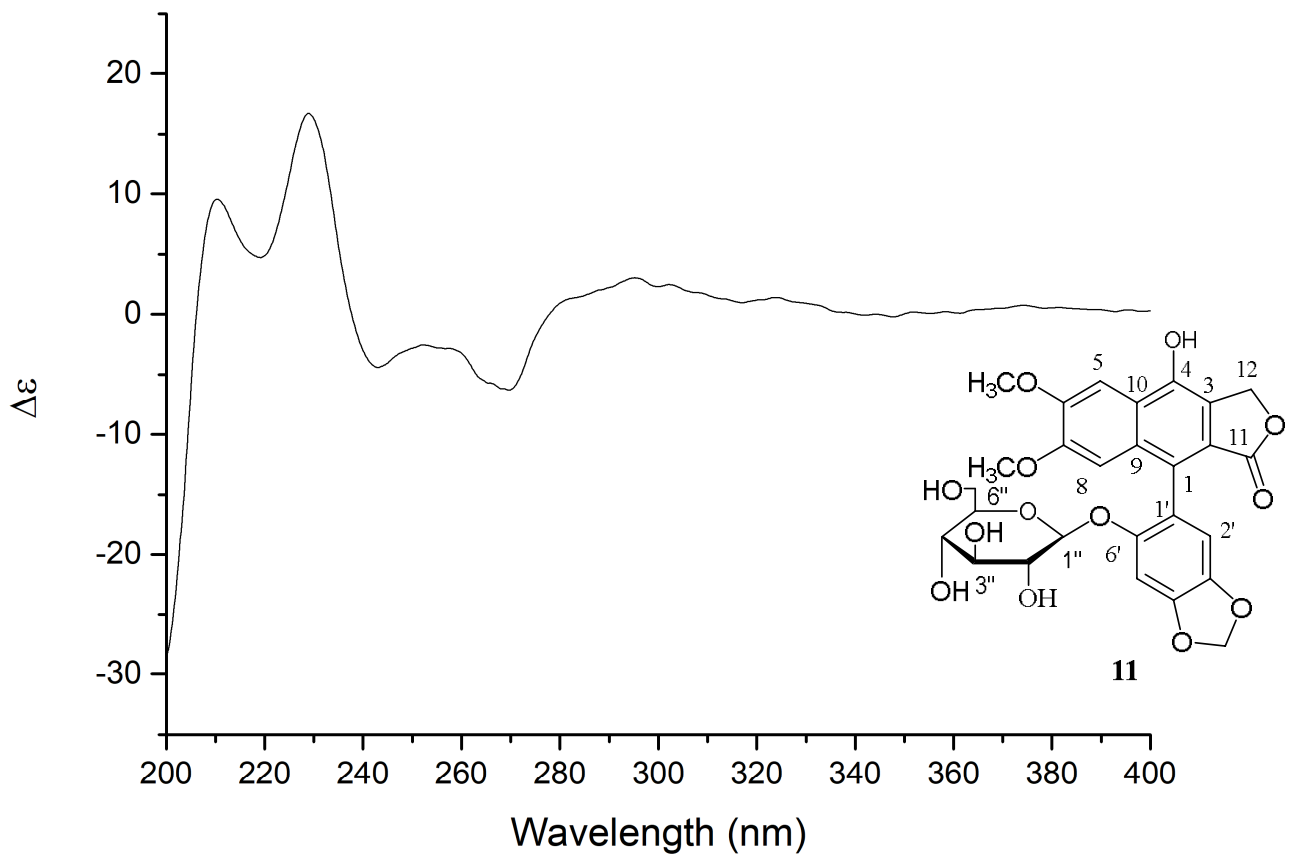

Figure S97. The CD Spectrum of Compound 11.

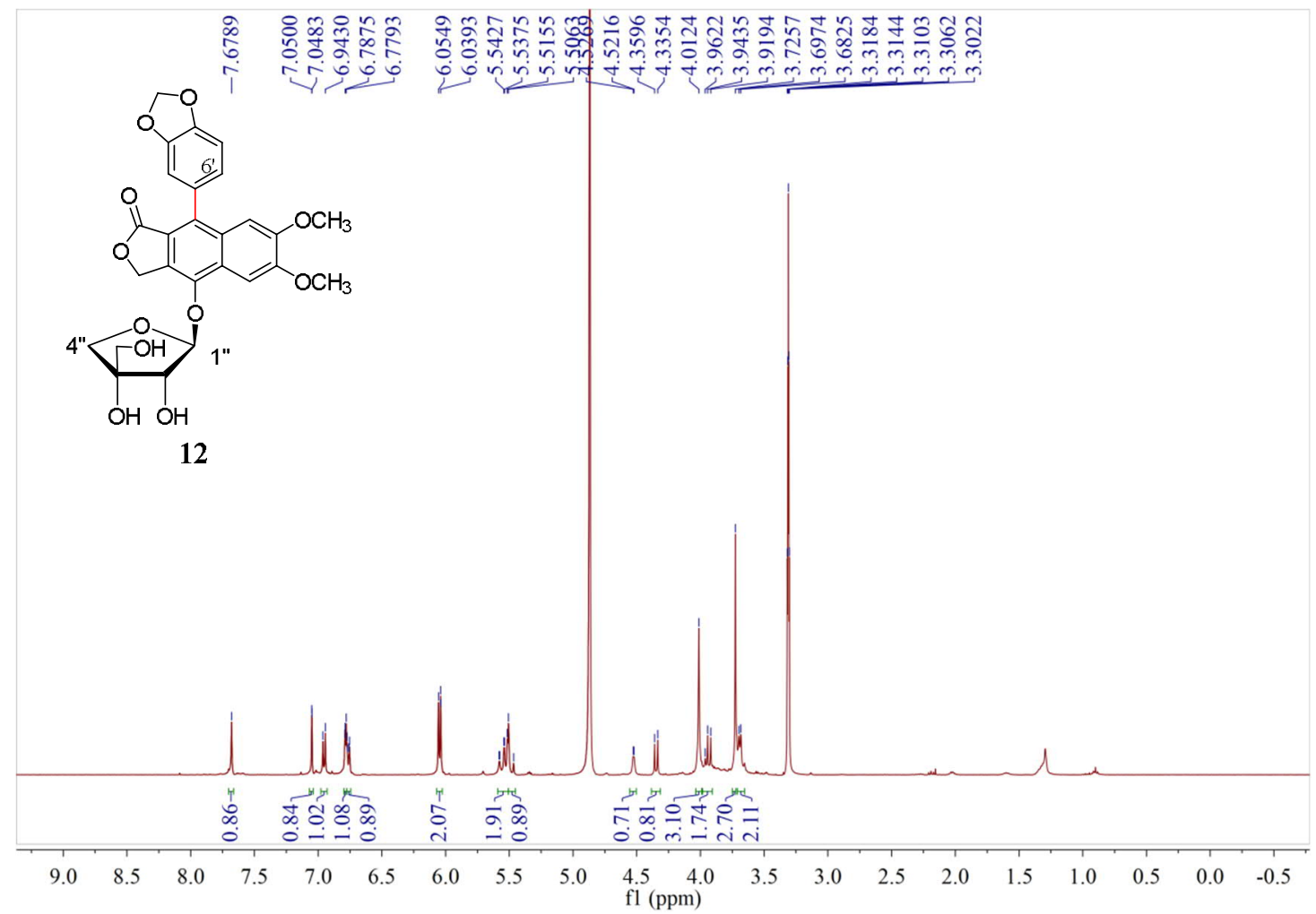

Figure S98. The ${ }^{1} \mathrm{H}$ NMR Spectrum of Compound 12 in $\mathrm{CD}_{3} \mathrm{OD}(400 \mathrm{MHz})$. 


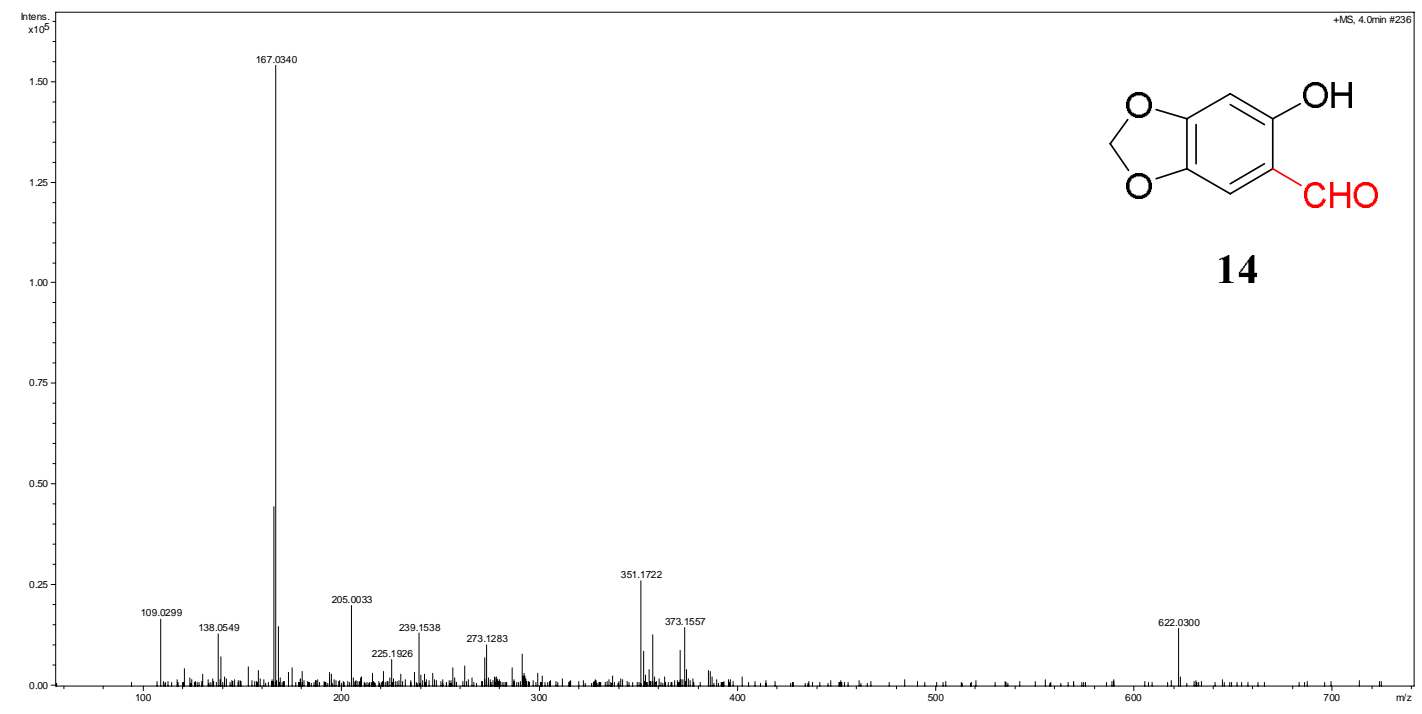

Figure S99. HRESIMS Data of intermediate Compound 14.

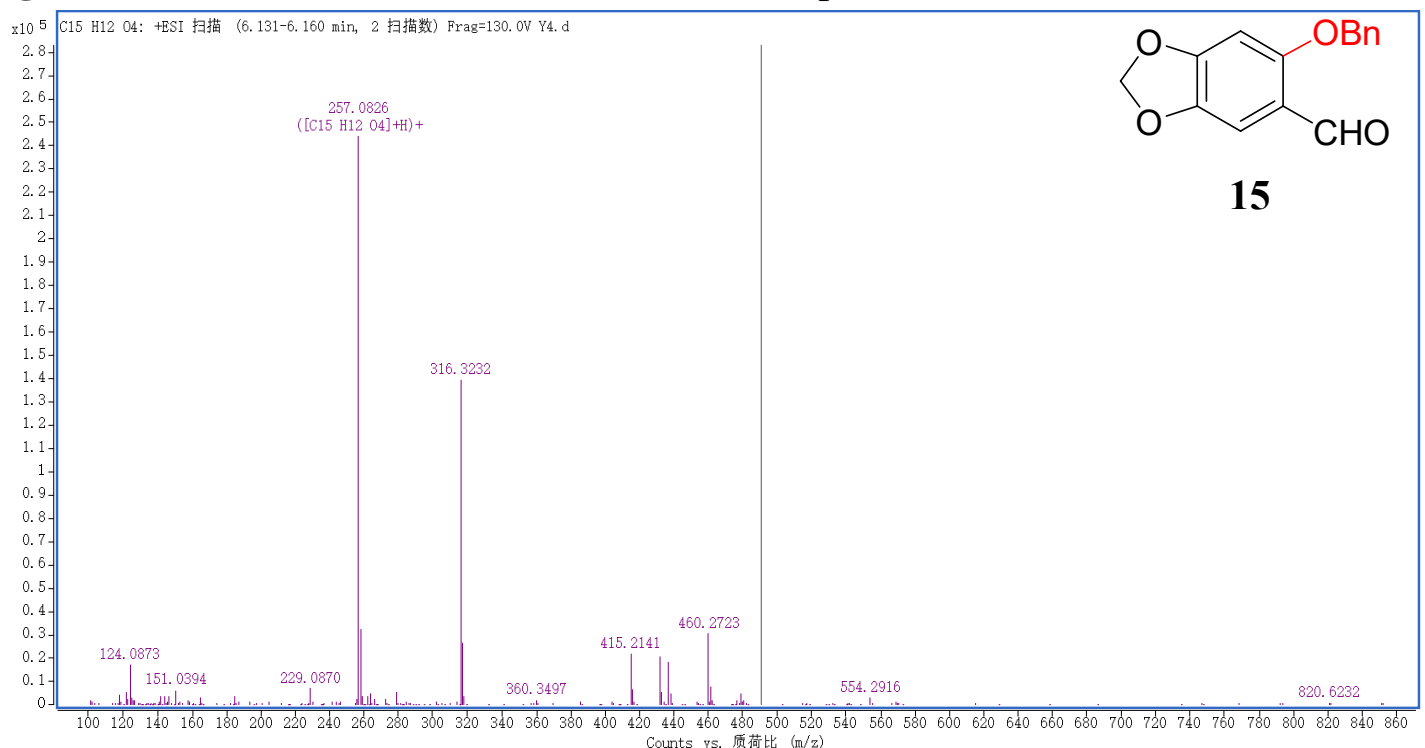

Figure S100. HRESIMS Data of intermediate Compound 15.

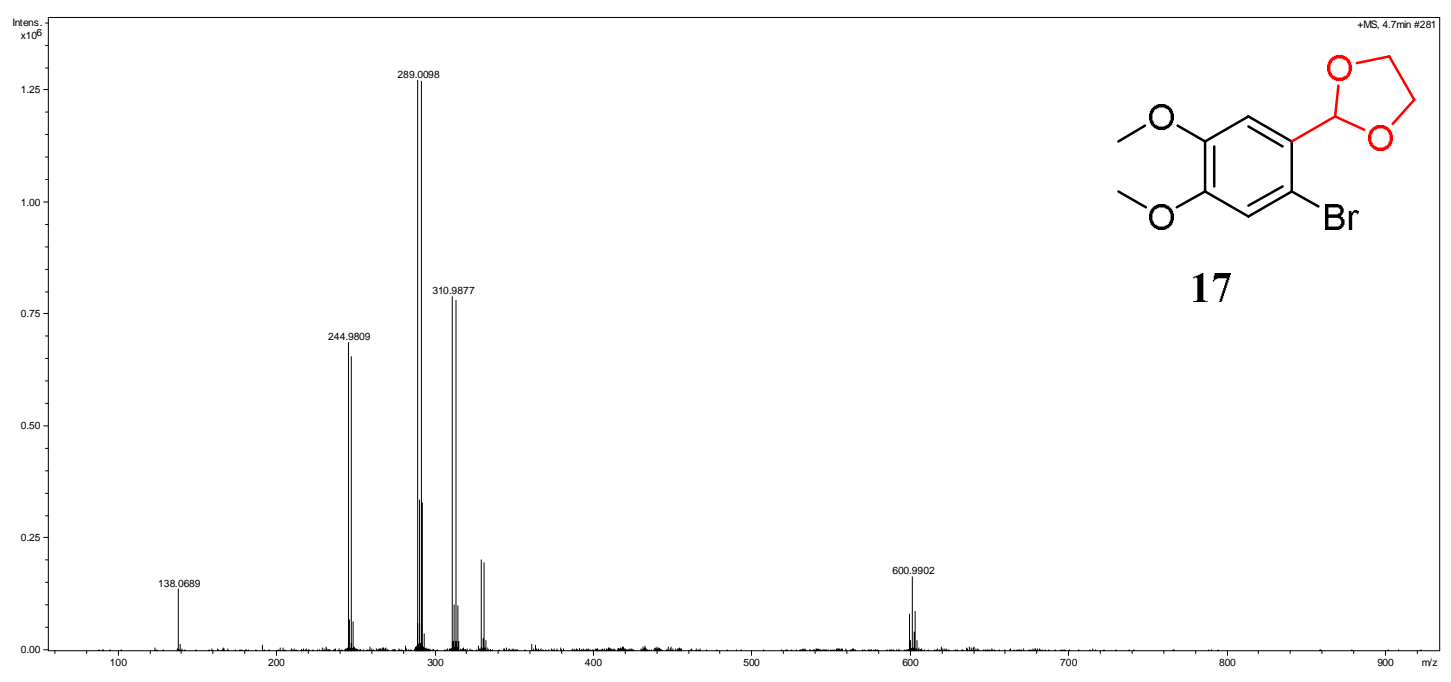

Figure S101. HRESIMS Data of intermediate Compound 17. 


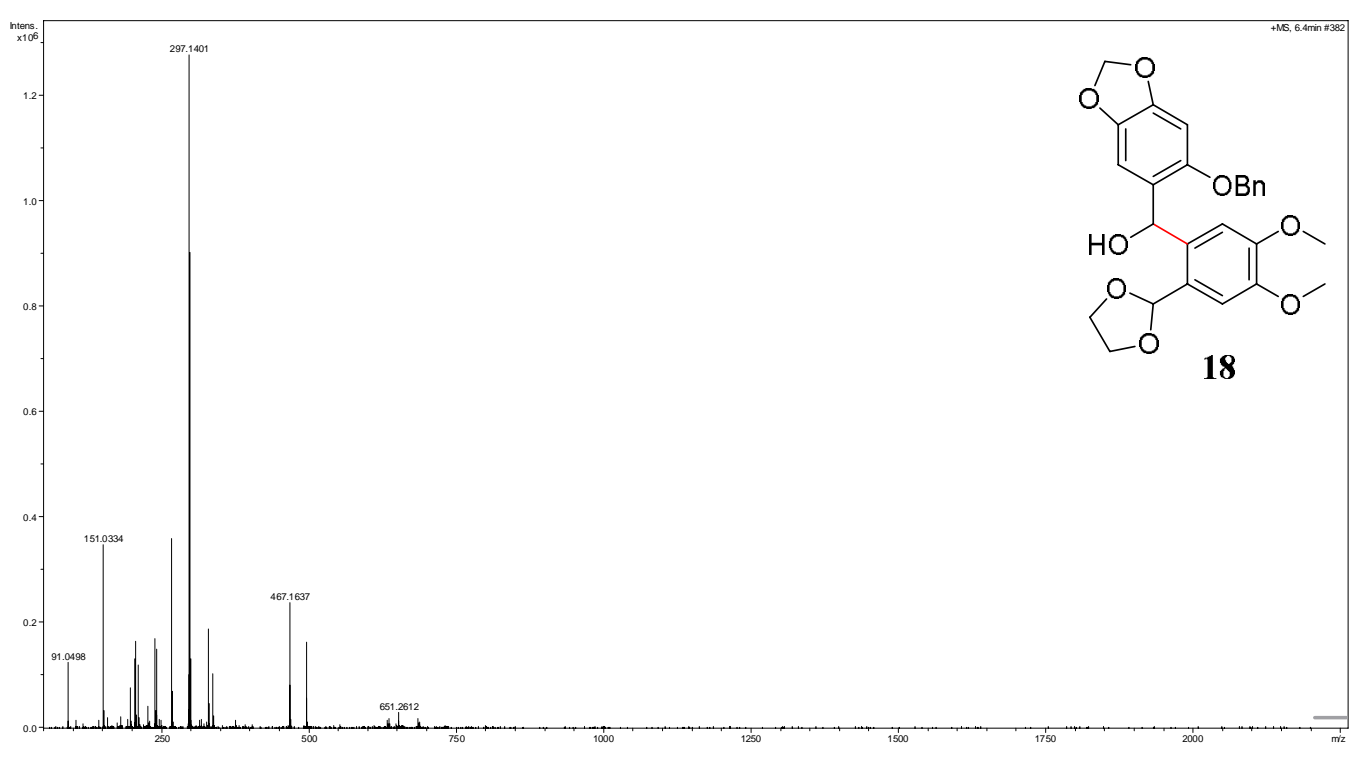

Figure S102. HRESIMS Data of intermediate Compound 18.

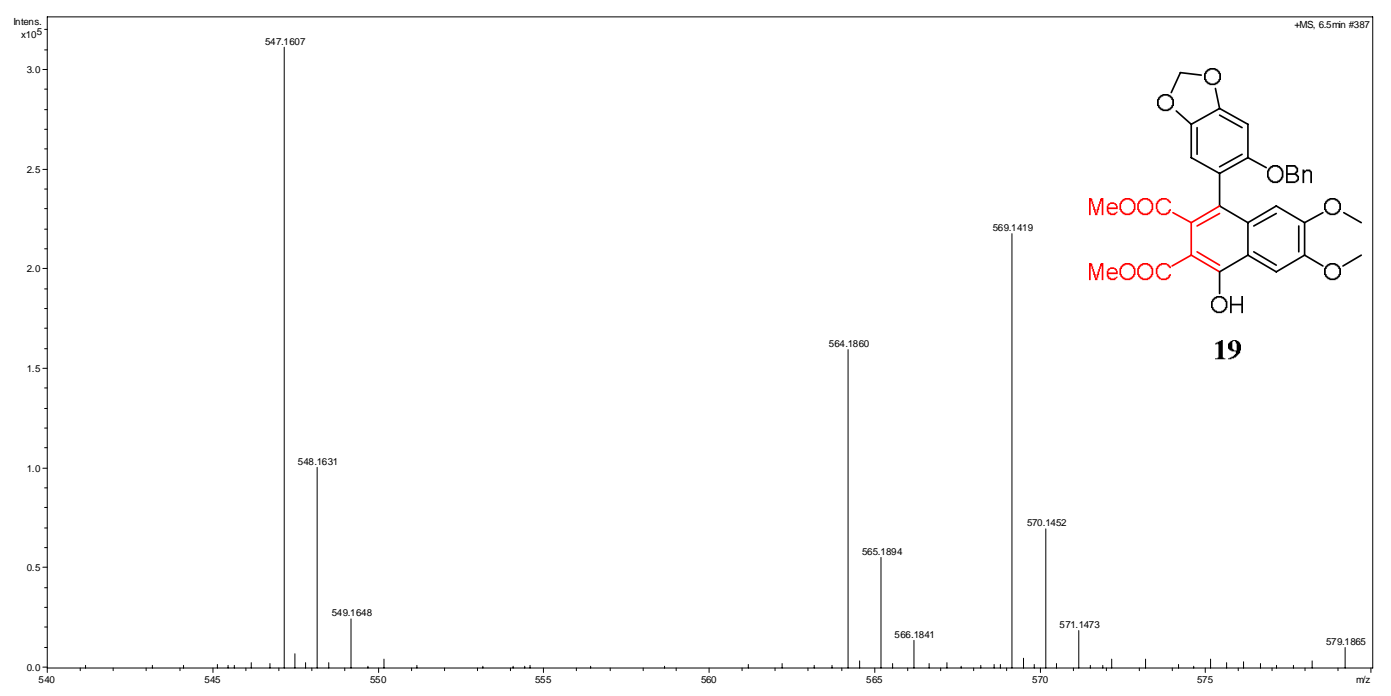

Figure S103. HRESIMS Data of intermediate Compound 19. 


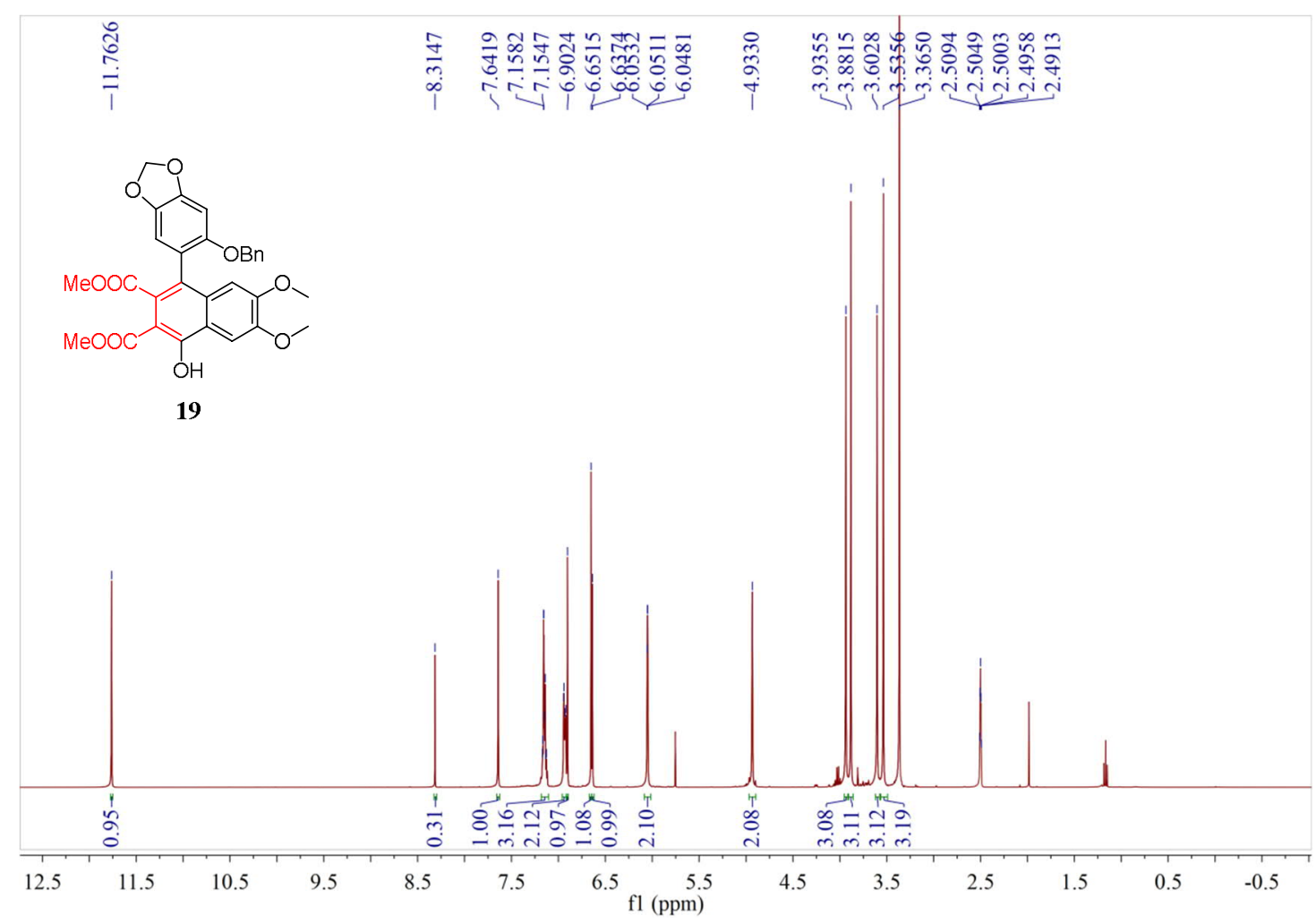

Figure S104. The ${ }^{1} \mathrm{H}$ NMR Spectrum of intermediate Compound 19 in DMSO- $d_{6}$ (400 MHz).

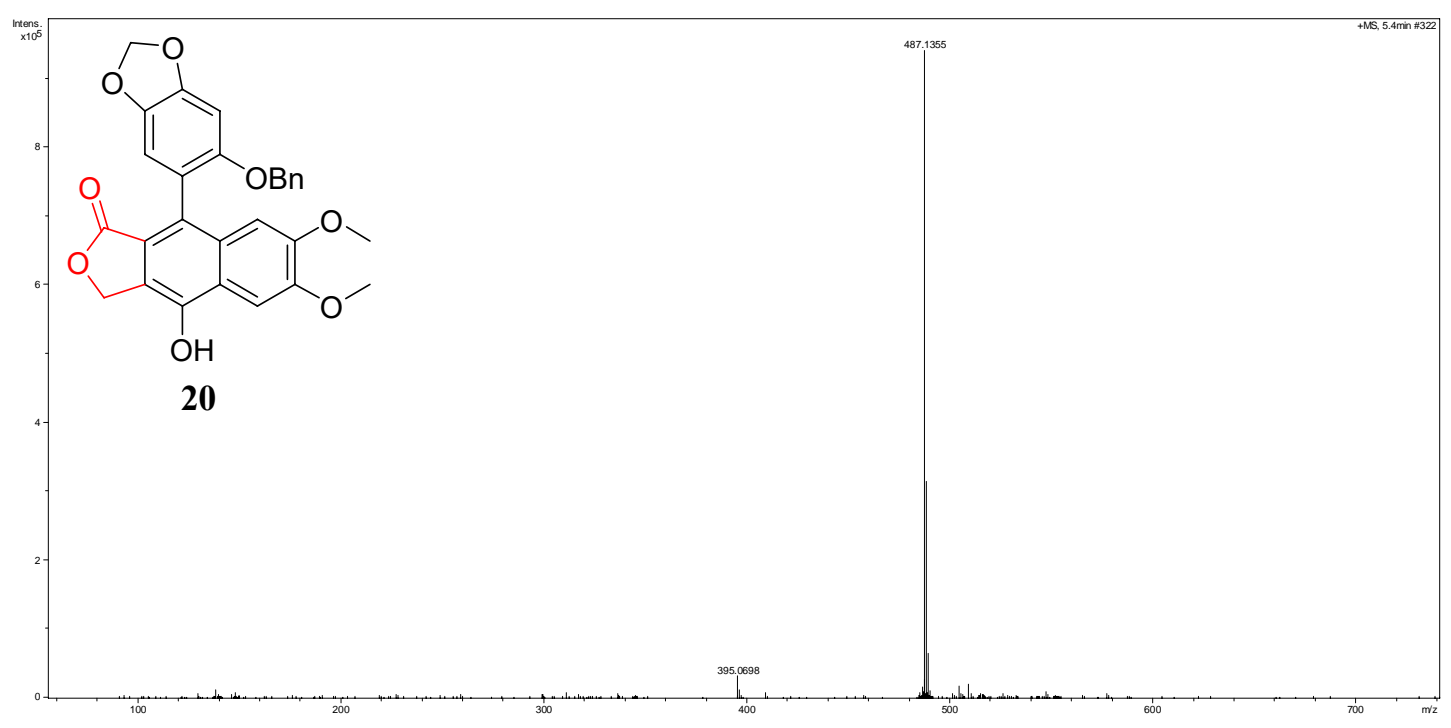

Figure S105. HRESIMS Data of intermediate Compound 20. 


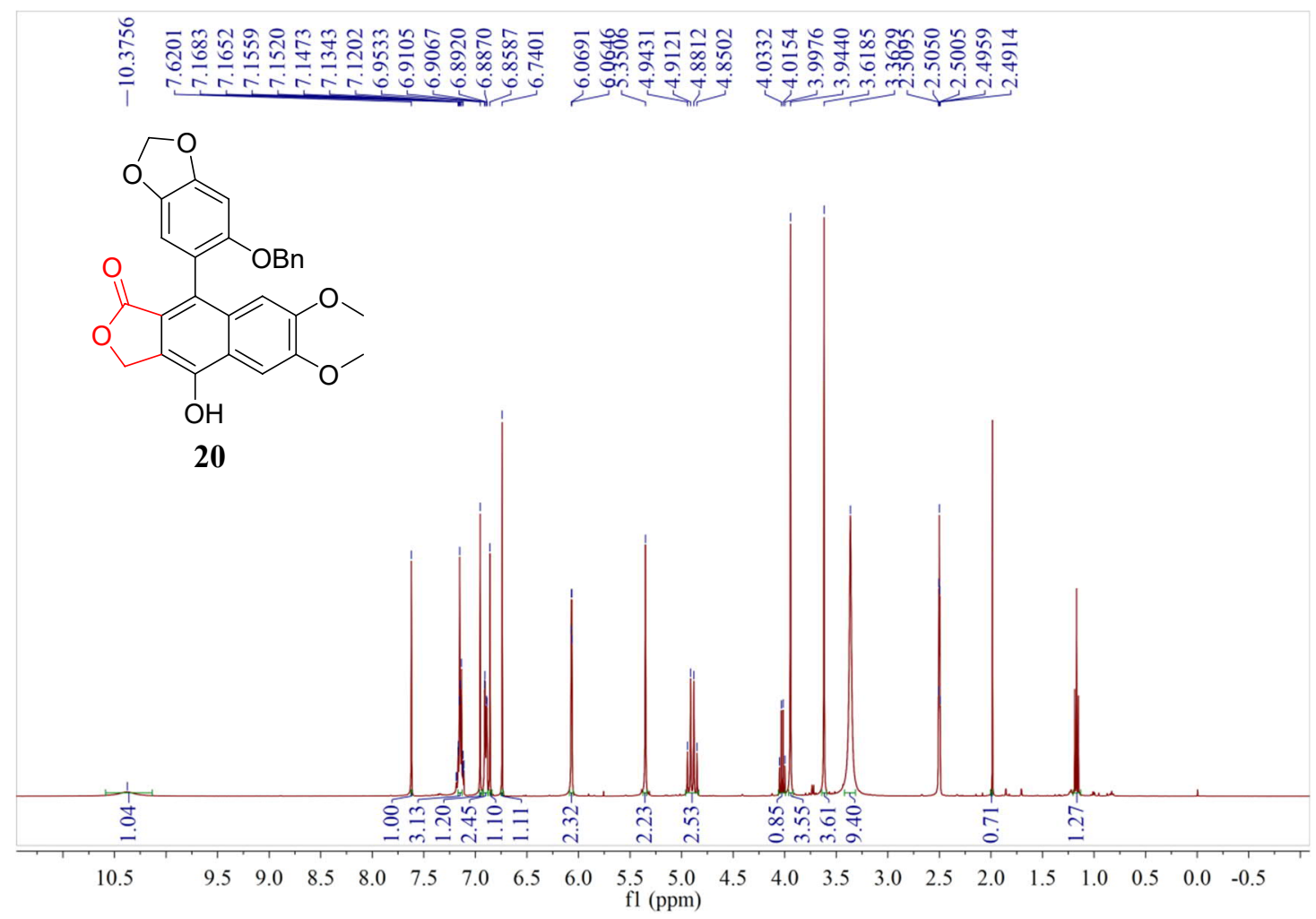

Figure S106. The ${ }^{1} \mathrm{H}$ NMR Spectrum of intermediate Compound 20 in DMSO- $d_{6}$ (400 MHz).

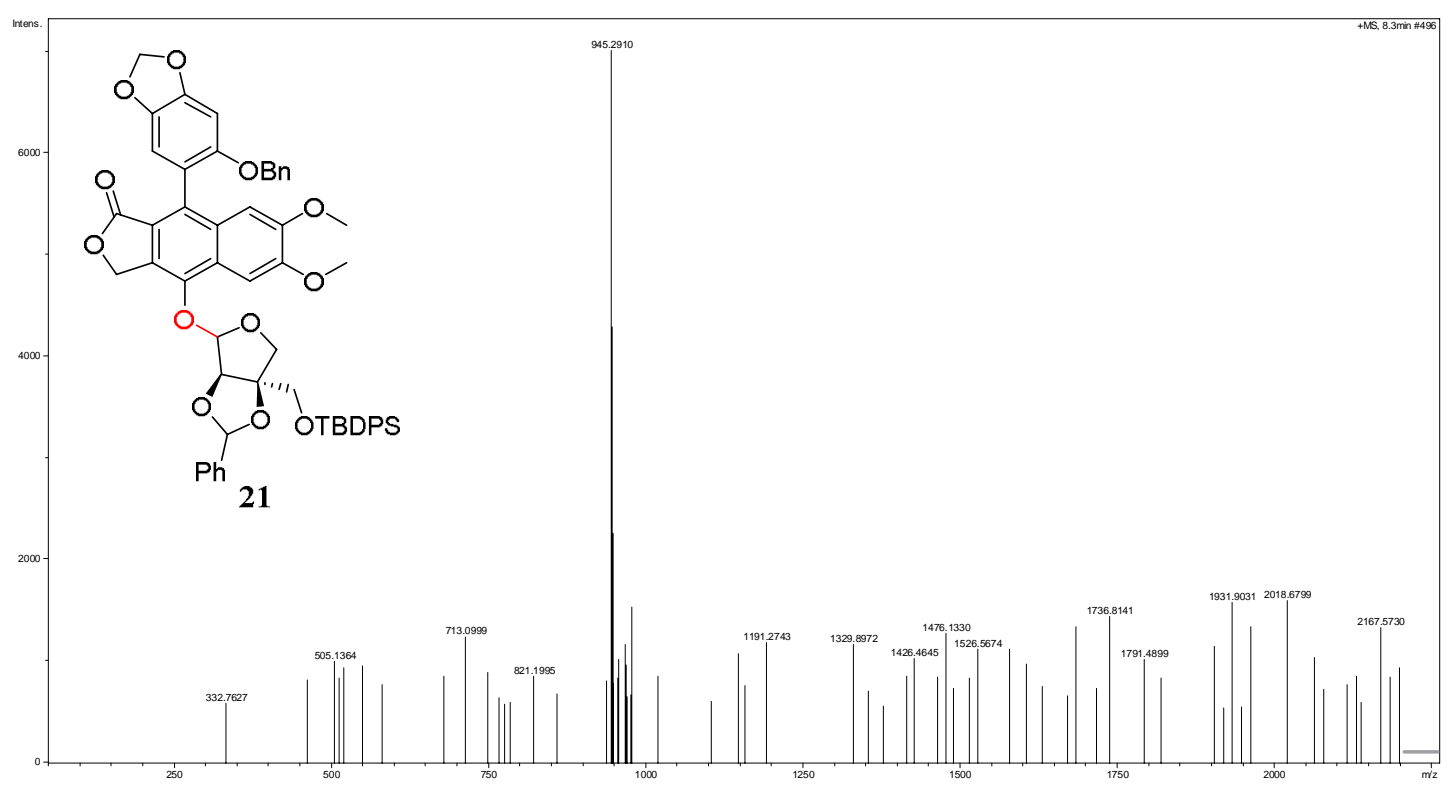

Figure S107. HRESIMS Data of intermediate Compound 21. 


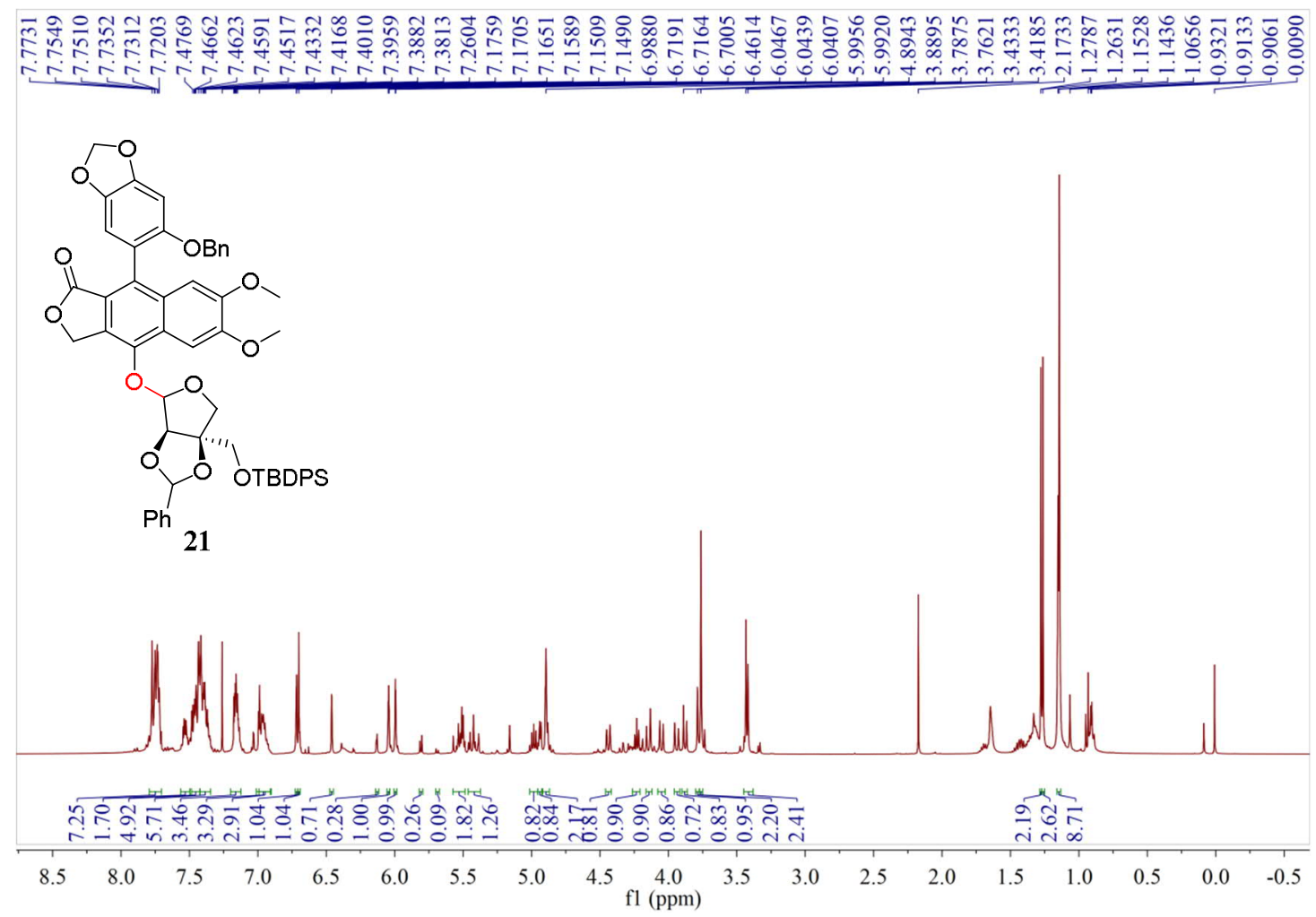

Figure S108. The ${ }^{1} \mathrm{H}$ NMR Spectrum of intermediate Compound 21 in $\mathrm{CDCl}_{3}(400$ $\mathrm{MHz})$.

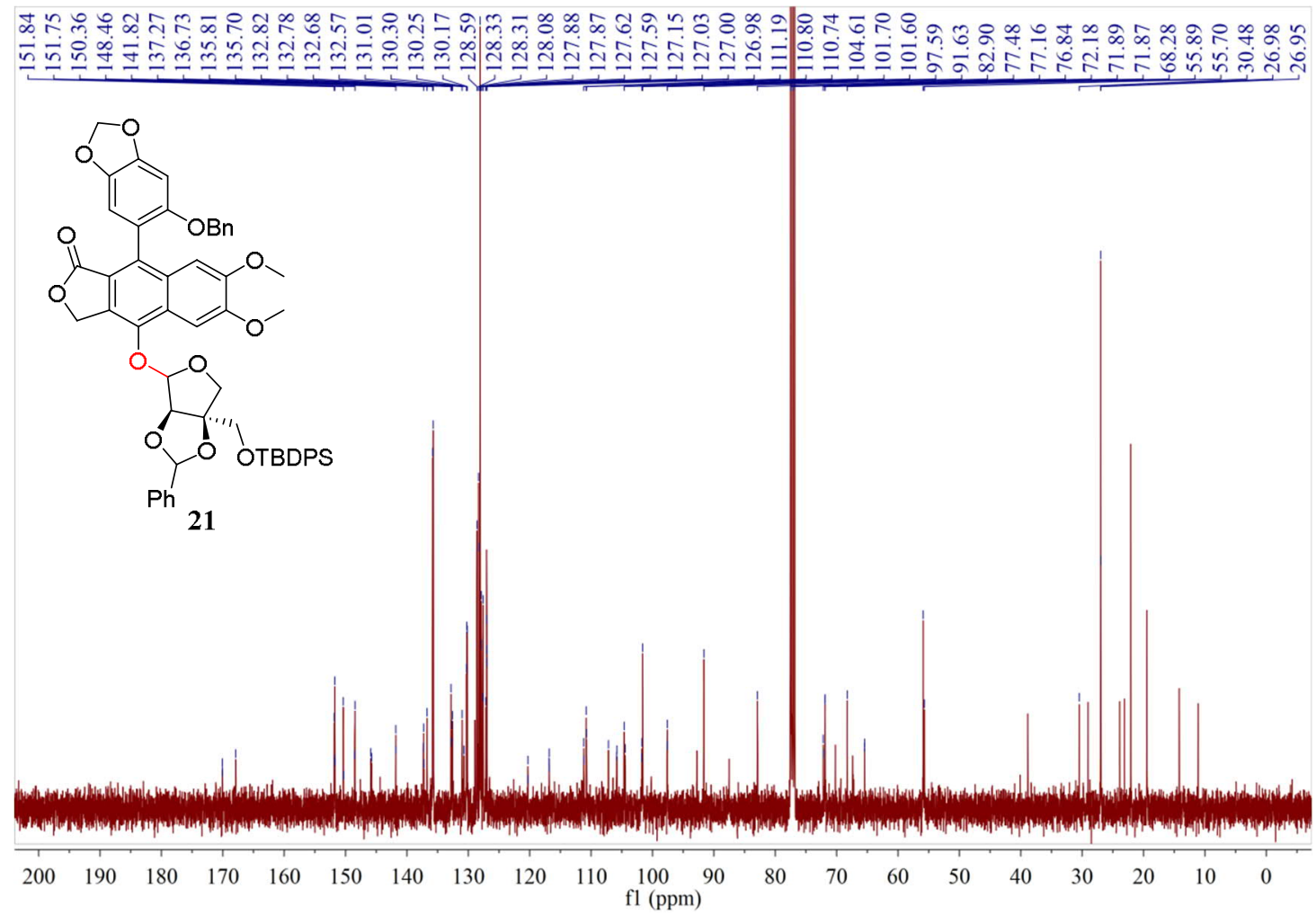

Figure S109. The ${ }^{13} \mathrm{C}\left\{{ }^{1} \mathrm{H}\right\}$ NMR Spectrum of intermediate Compound 21 in $\mathrm{CDCl}_{3}$ (100 MHz). 


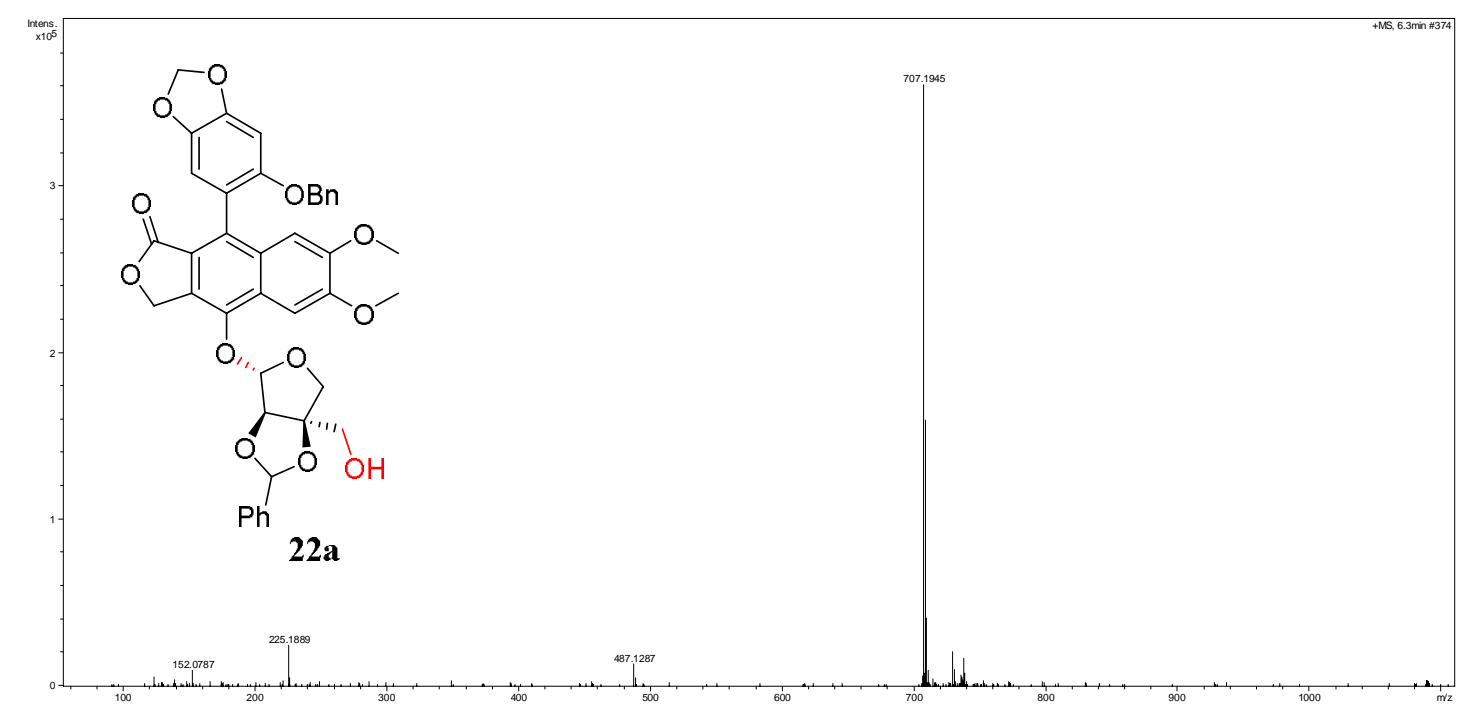

Figure S110. HRESIMS Data of intermediate Compound 22a.

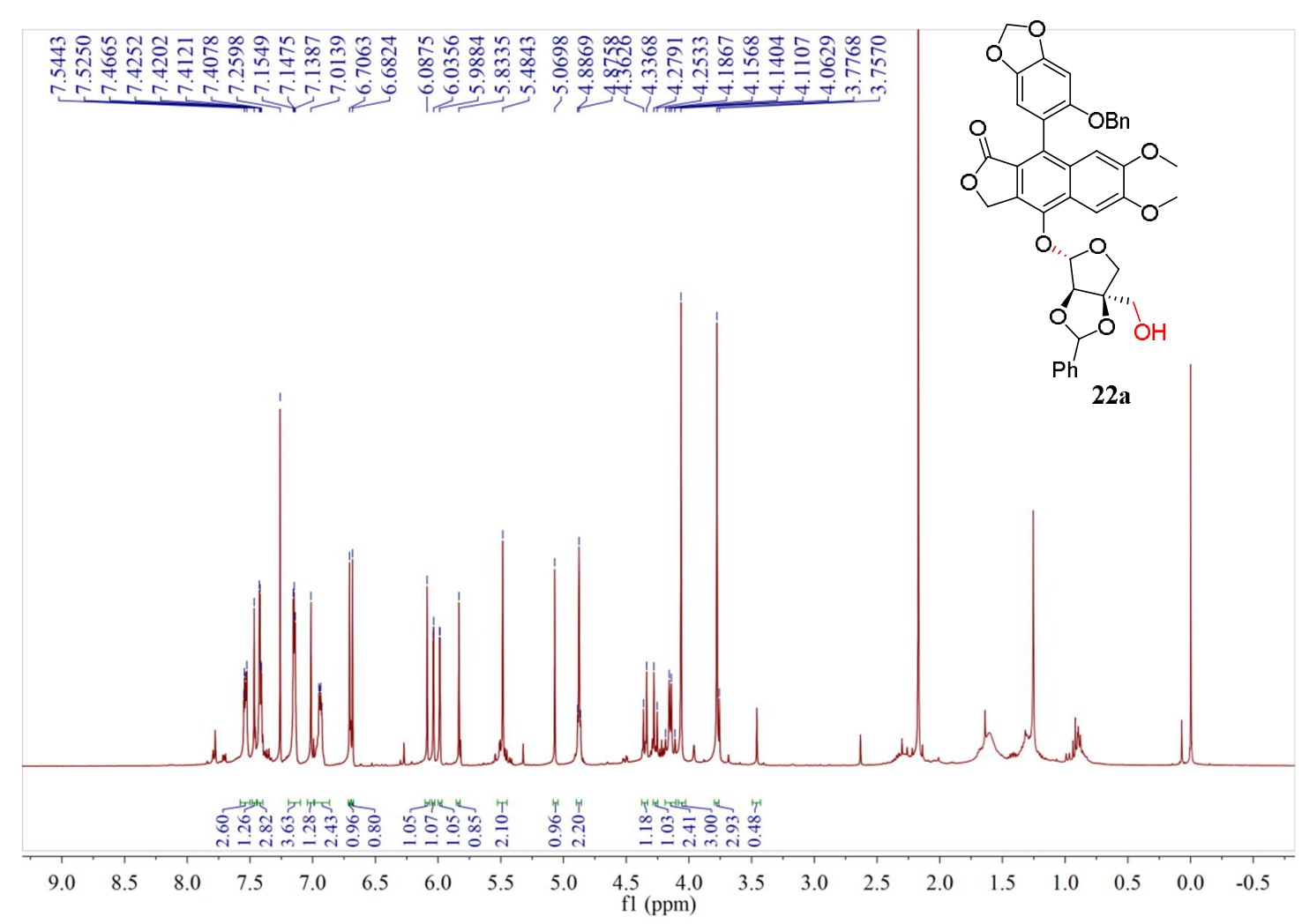

Figure S111. The ${ }^{1} \mathrm{H}$ NMR Spectrum of intermediate Compound 22a in $\mathrm{CDCl}_{3}(400$ $\mathrm{MHz}$ ). 


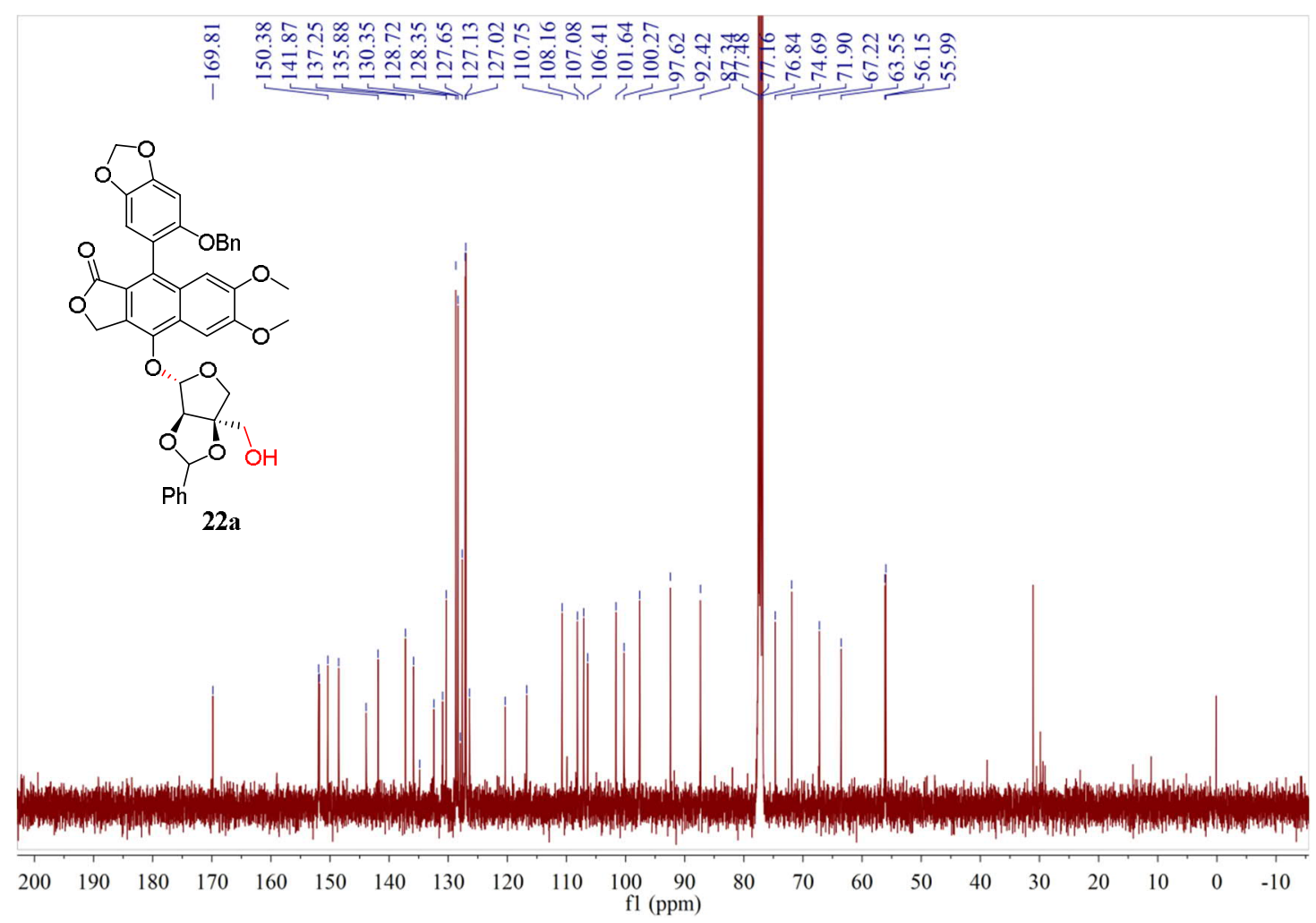

Figure S112. The ${ }^{13} \mathrm{C}\left\{{ }^{1} \mathrm{H}\right\}$ NMR Spectrum of intermediate Compound 22a in $\mathrm{CDCl}_{3}$ (100 MHz).

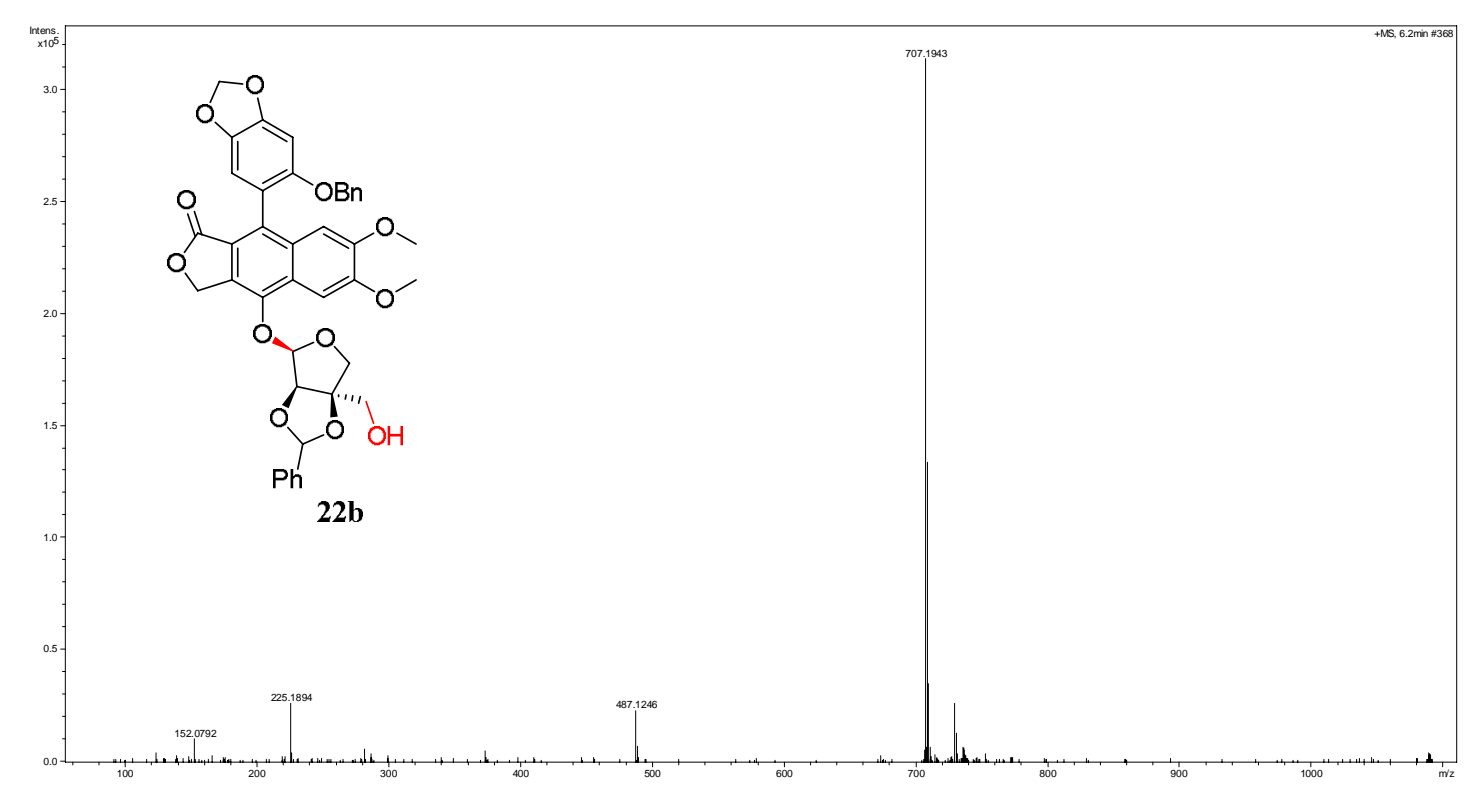

Figure S113. HRESIMS Data of intermediate Compound 22b. 


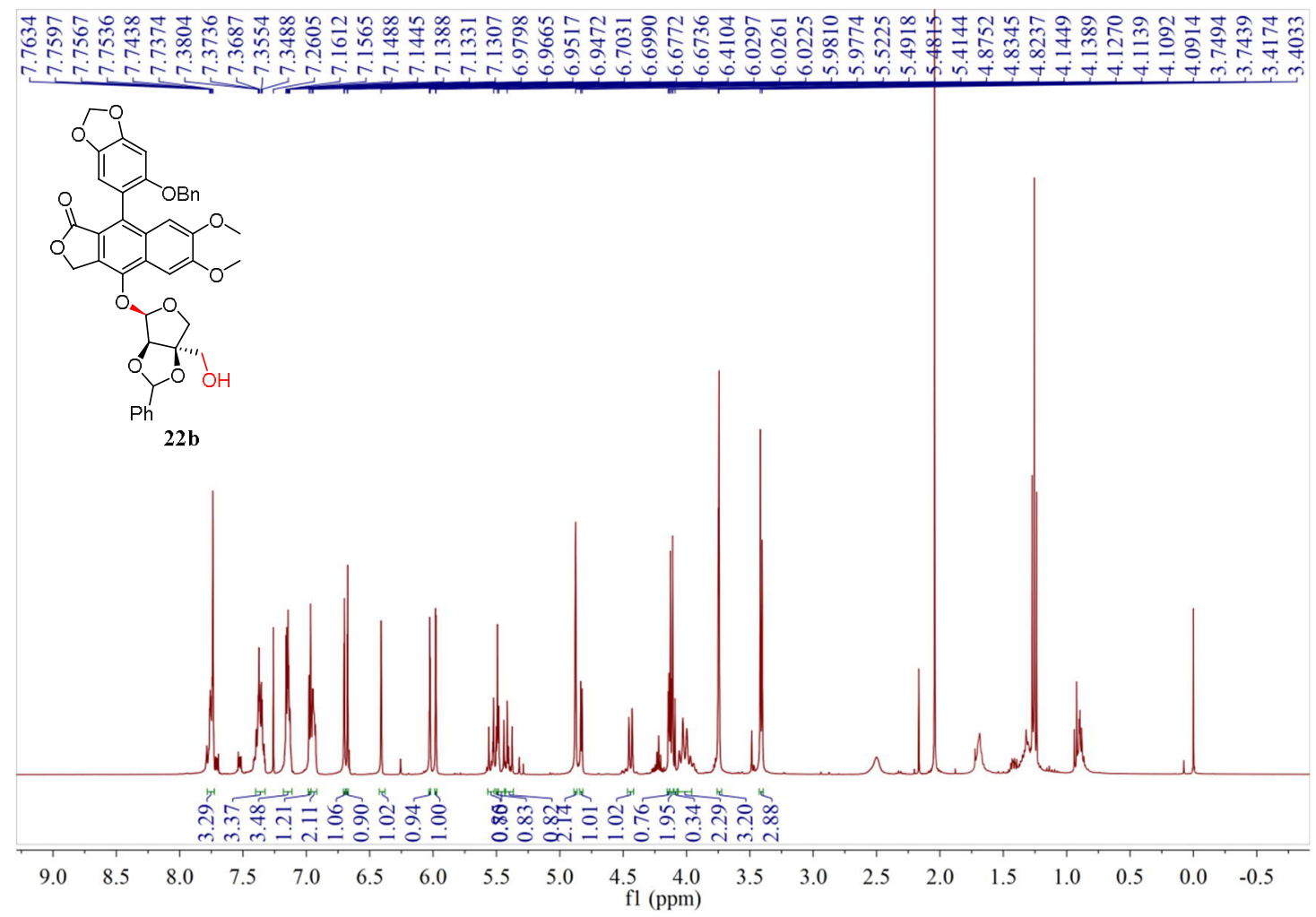

Figure S114. The ${ }^{1} \mathrm{H}$ NMR Spectrum of intermediate Compound 22b in $\mathrm{CDCl}_{3}(400$ $\mathrm{MHz})$.

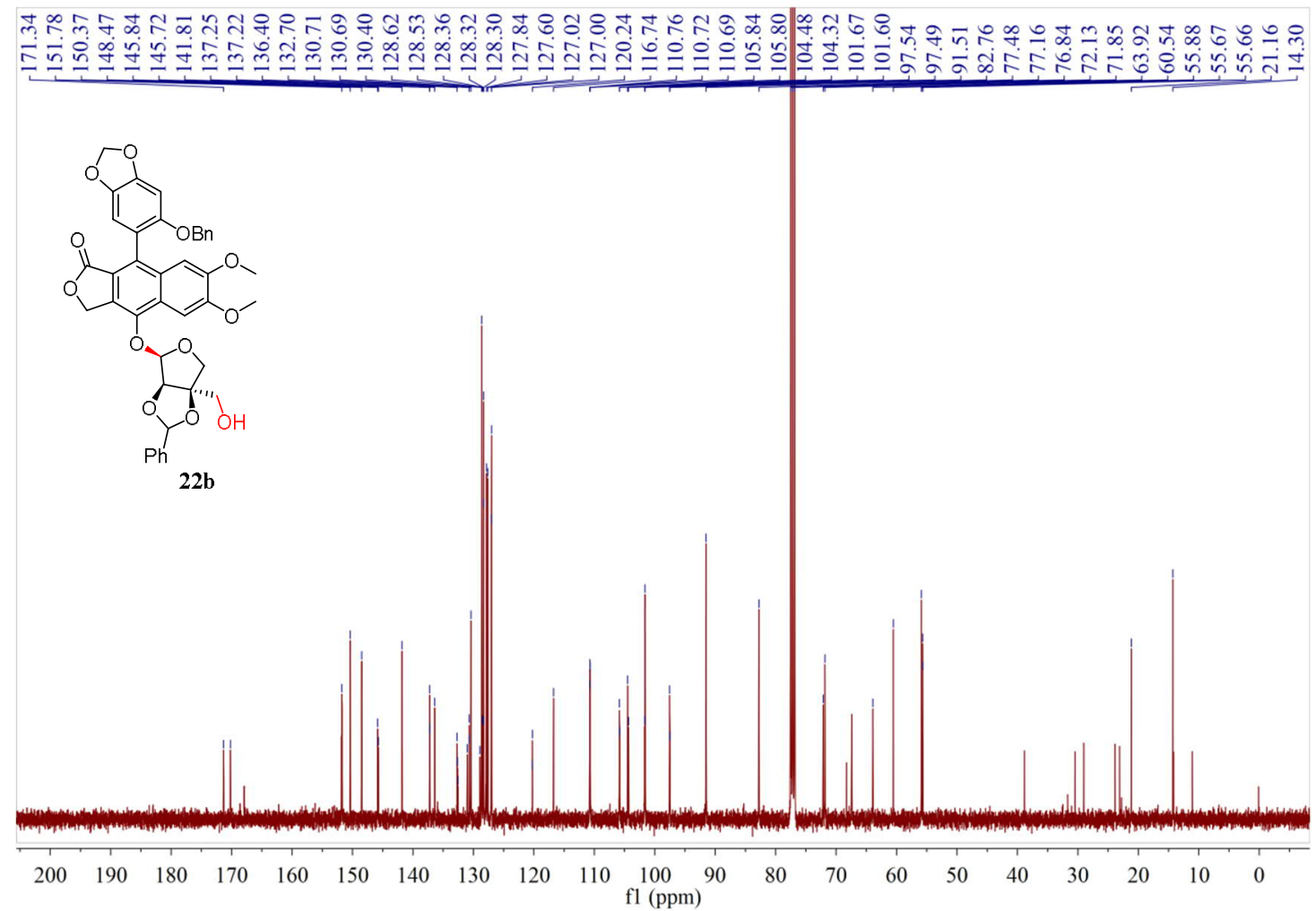

Figure S115. The ${ }^{13} \mathrm{C}\left\{{ }^{1} \mathrm{H}\right\}$ NMR Spectrum of intermediate Compound 22b in $\mathrm{CDCl}_{3}$ (100 MHz). 


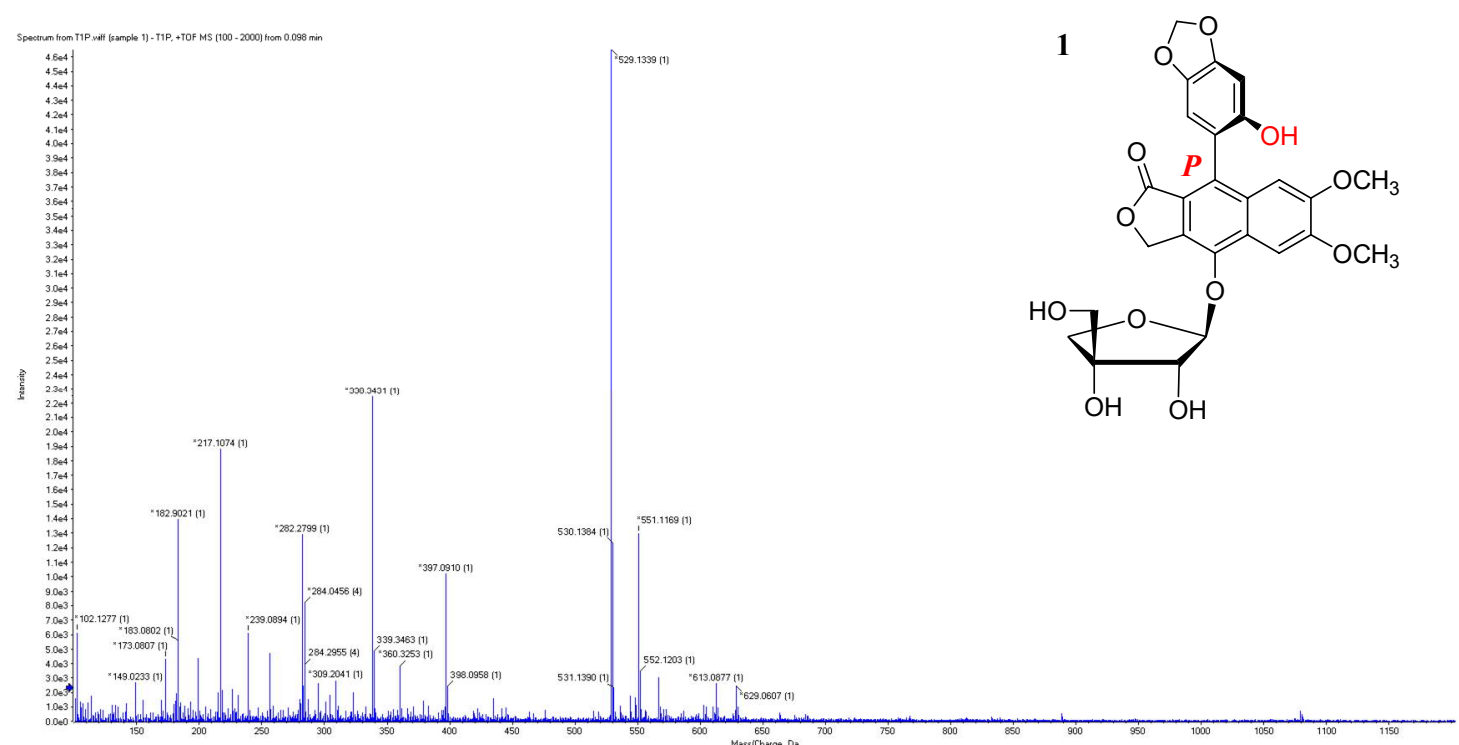

Figure S116. HRESIMS Data of synthetic 1.

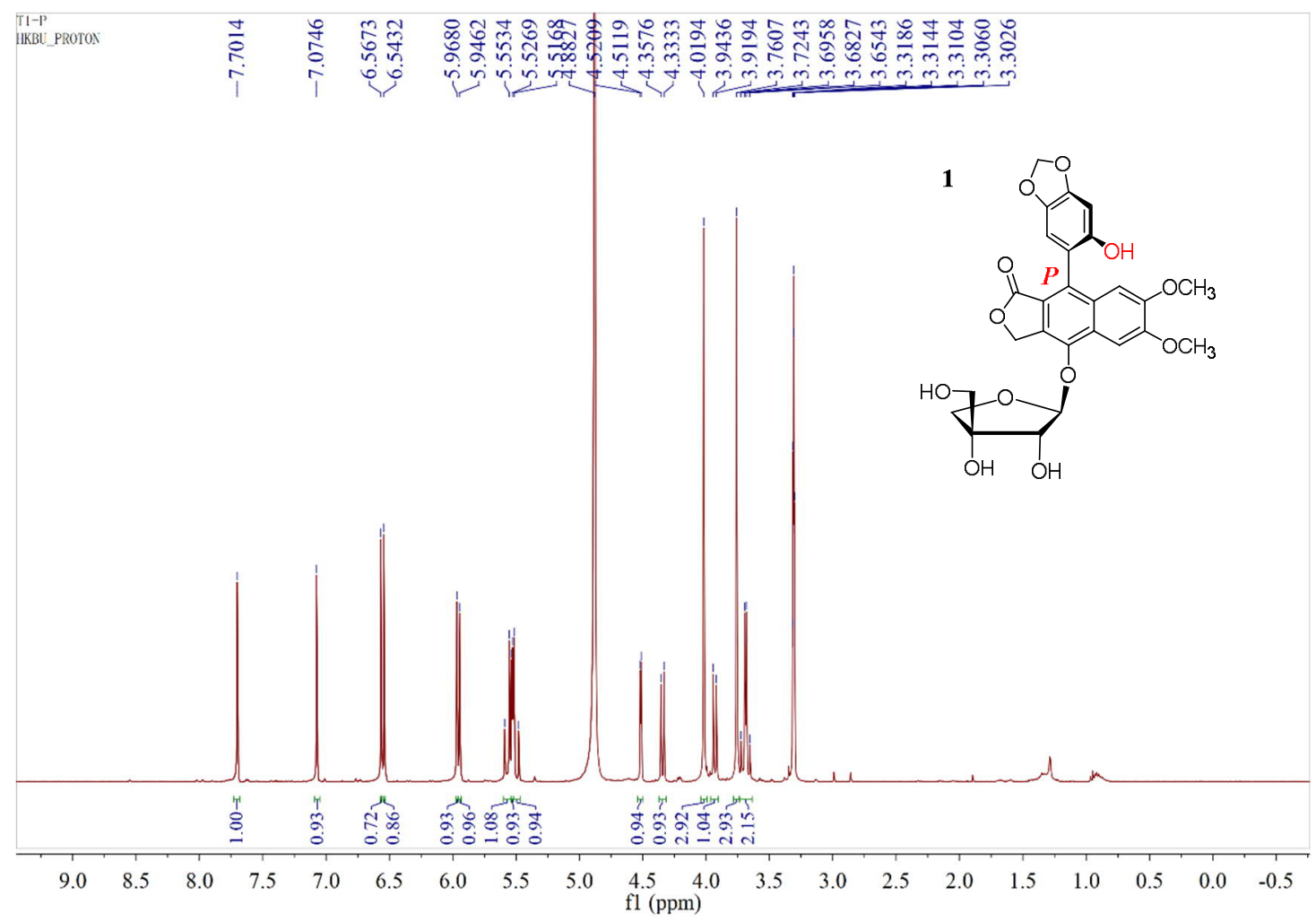

Figure S117. The ${ }^{1} \mathrm{H}$ NMR Spectrum of synthetic 1 in $\mathrm{CD}_{3} \mathrm{OD}(400 \mathrm{MHz})$. 


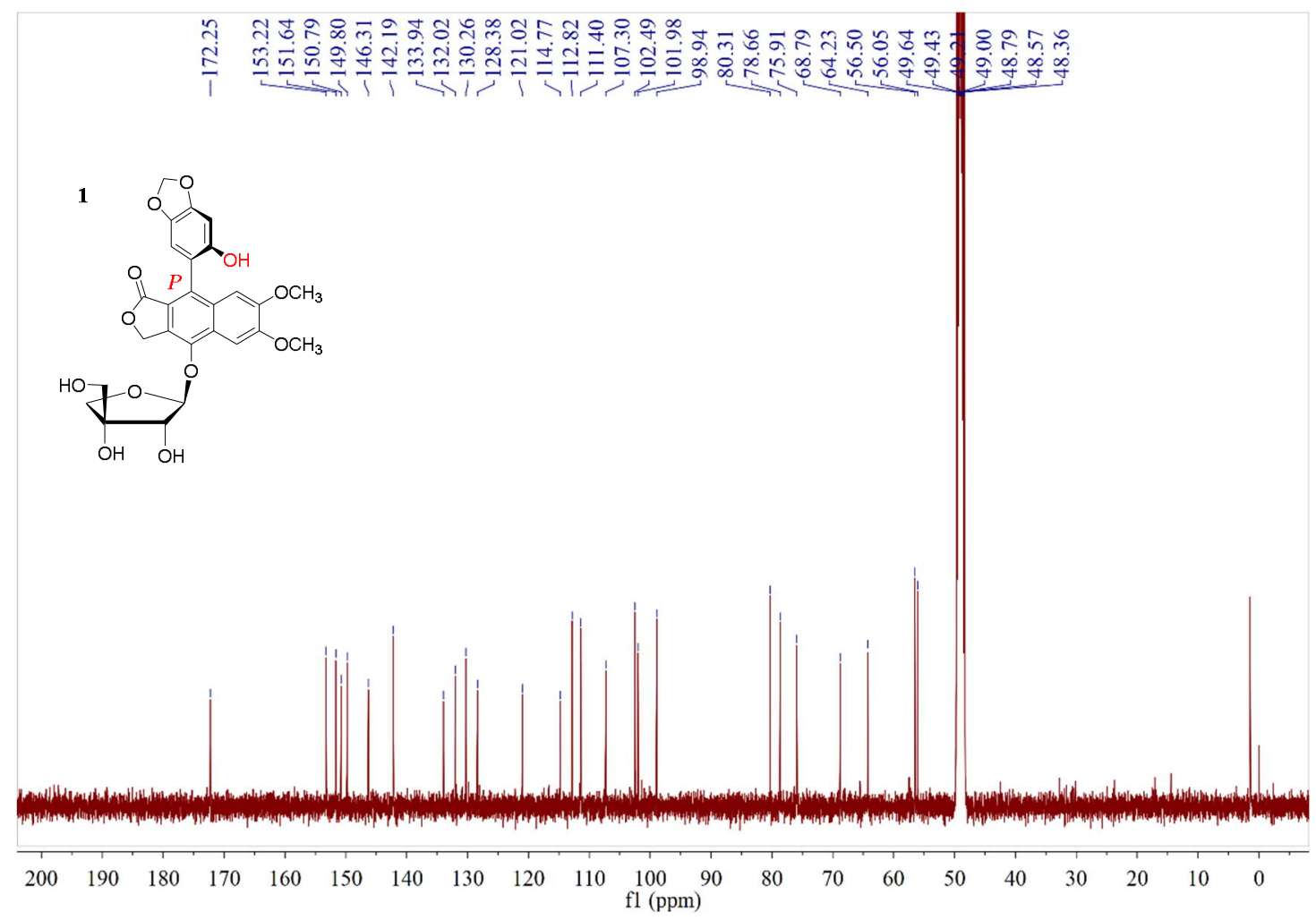

Figure S118. The ${ }^{13} \mathrm{C}\left\{{ }^{1} \mathrm{H}\right\}$ NMR Spectrum of synthetic 1 in $\mathrm{CD}_{3} \mathrm{OD}(100 \mathrm{MHz})$.

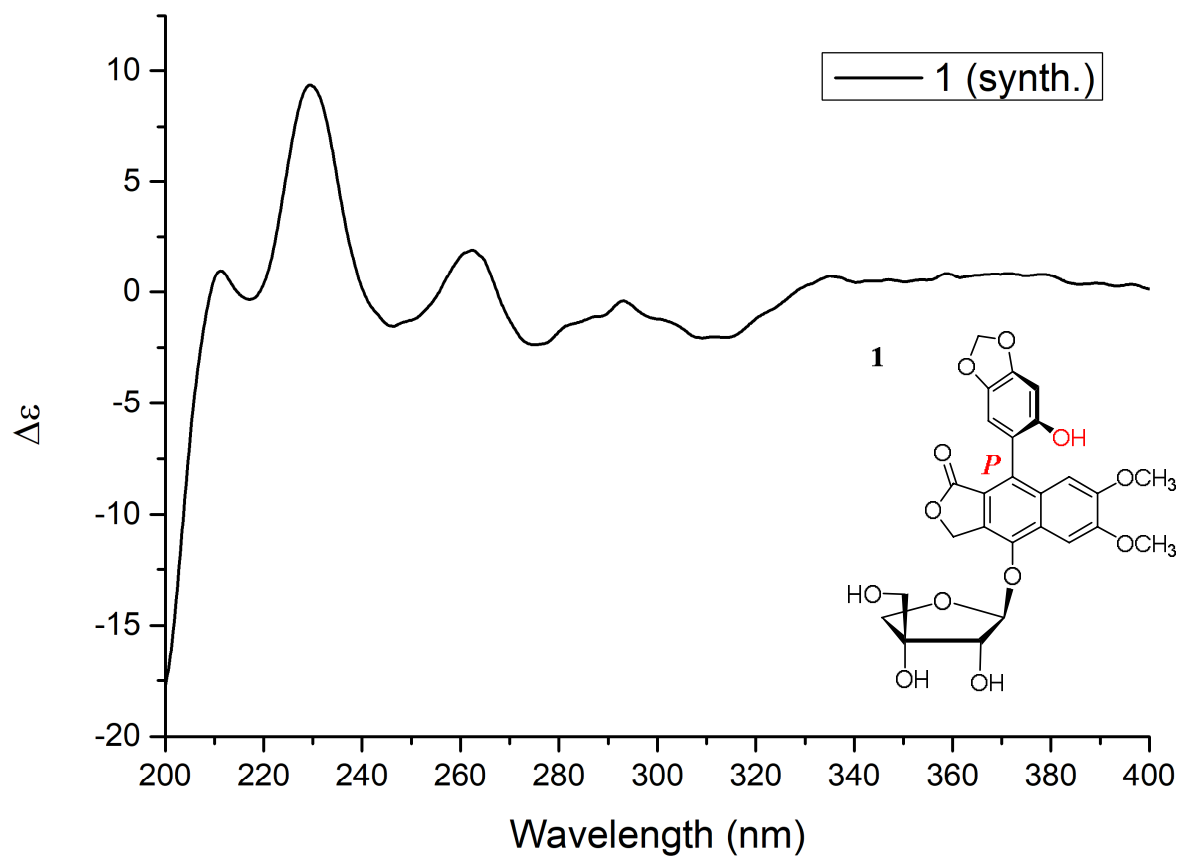

Figure S119. The CD Spectrum of synthetic 1 in $\mathrm{MeOH}$. 


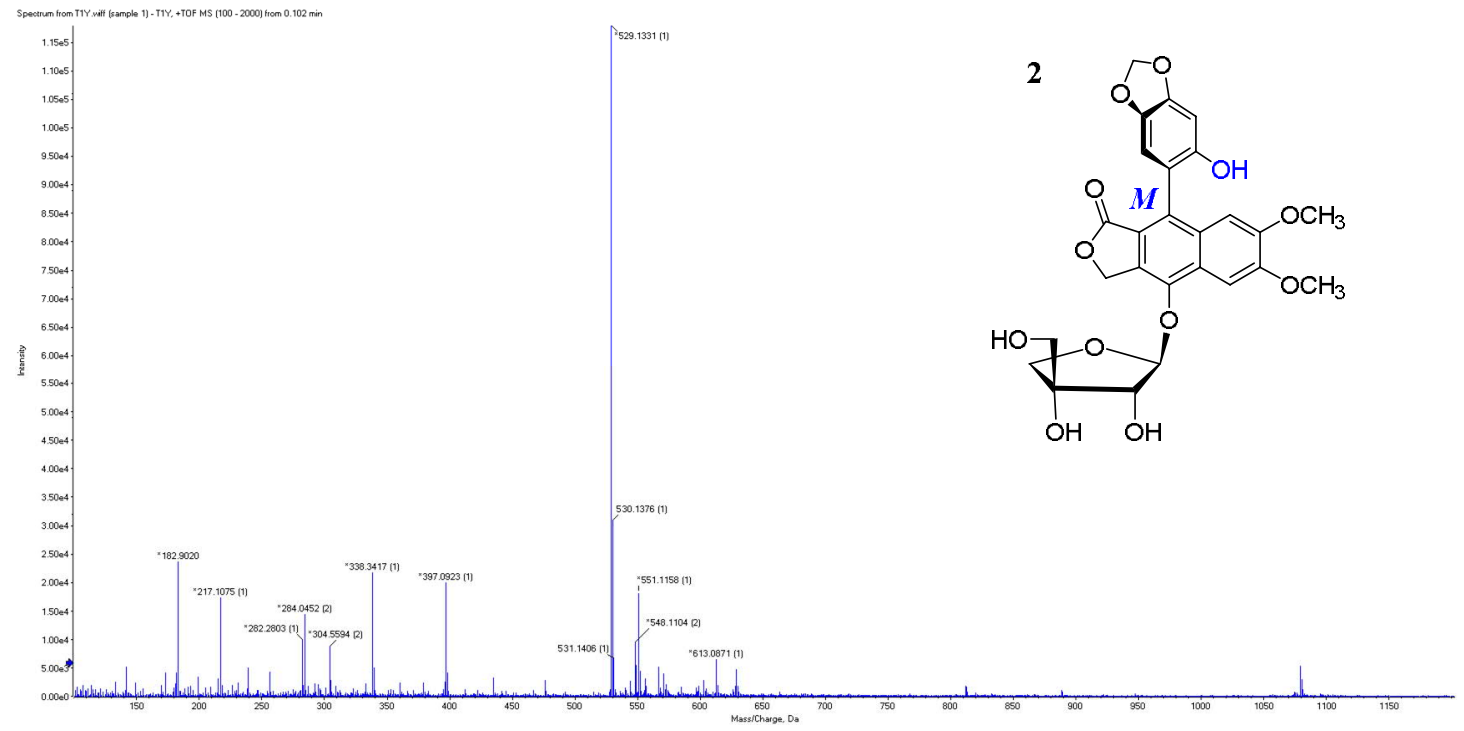

Figure S120. HRESIMS Data of synthetic 2.

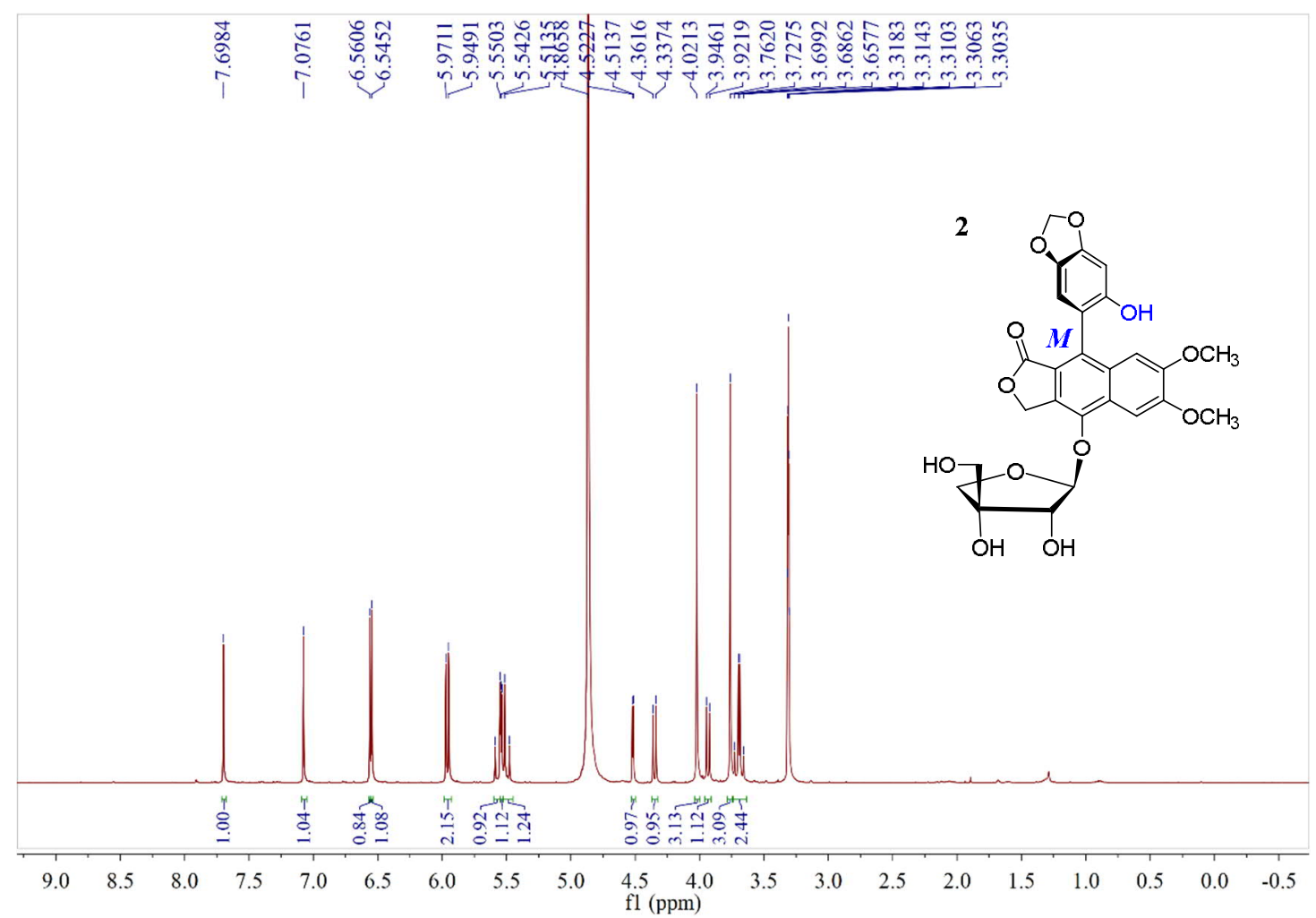

Figure S121. The ${ }^{1} \mathrm{H}$ NMR Spectrum of synthetic 2 in $\mathrm{CD}_{3} \mathrm{OD}(400 \mathrm{MHz})$. 


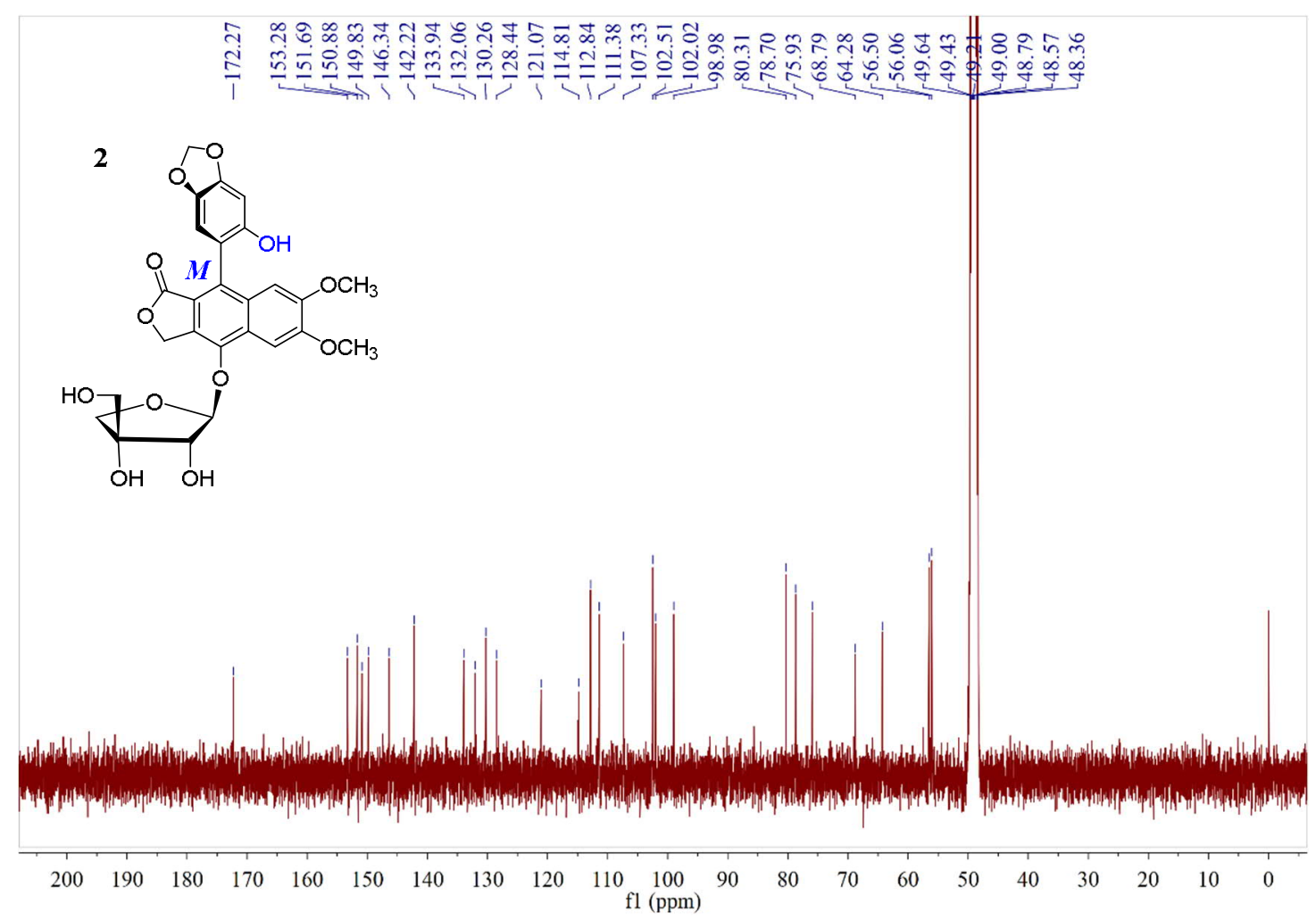

Figure S122. The ${ }^{13} \mathrm{C}$ NMR Spectrum of synthetic 2 in $\mathrm{CD}_{3} \mathrm{OD}(100 \mathrm{MHz})$.

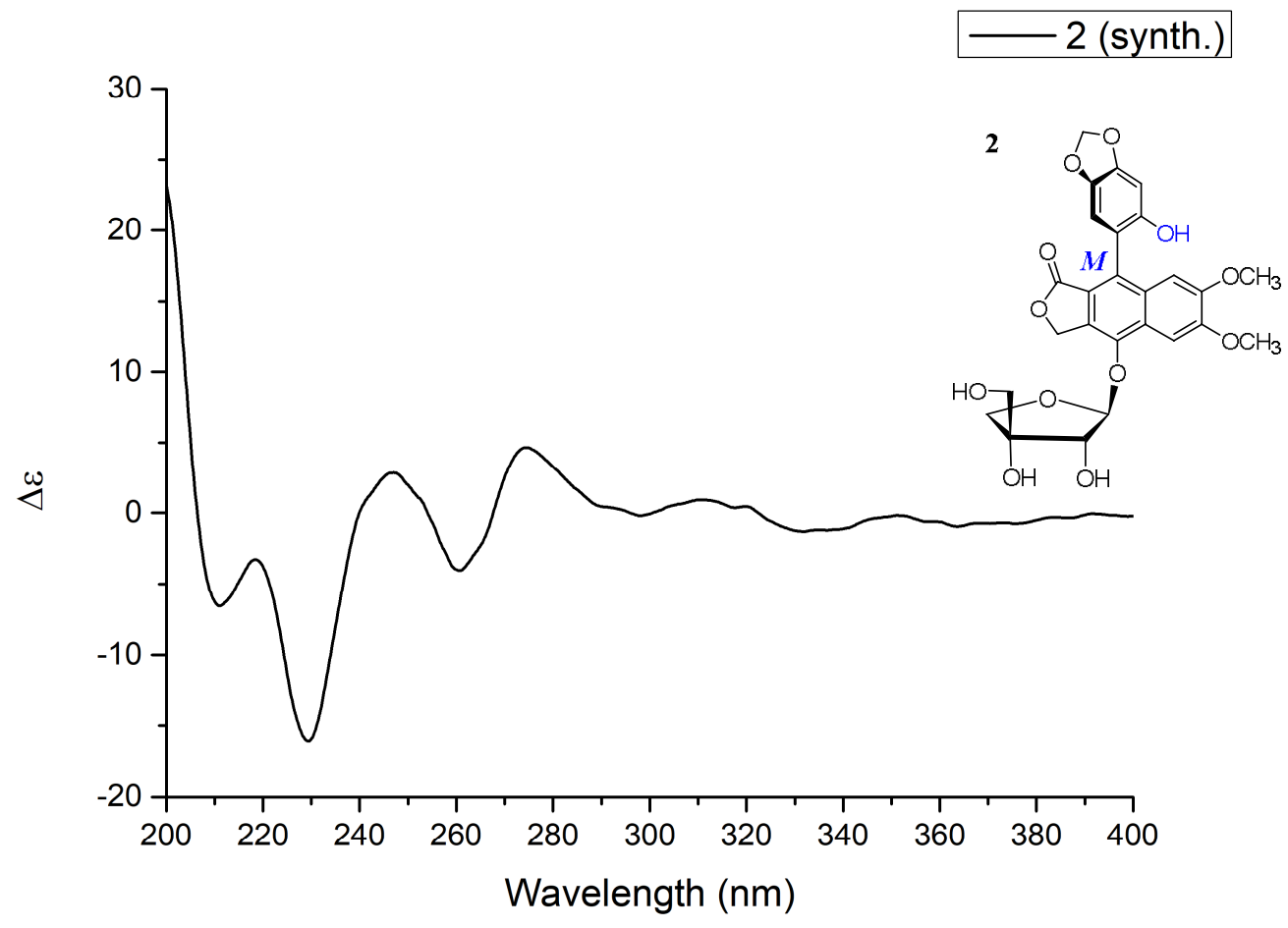

Figure S123. The CD Spectrum of synthetic 2 in $\mathrm{MeOH}$. 


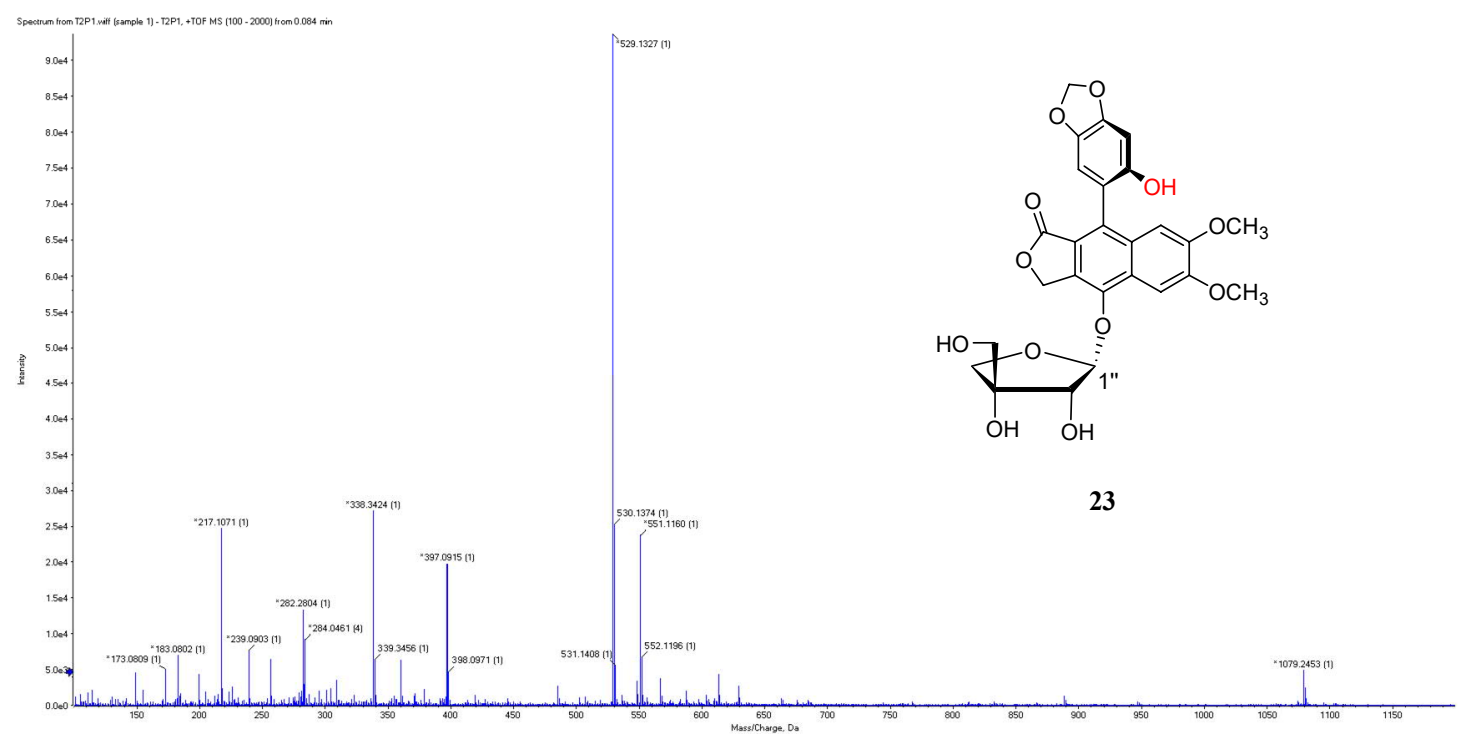

Figure S124. HRESIMS Data of synthetic 23.

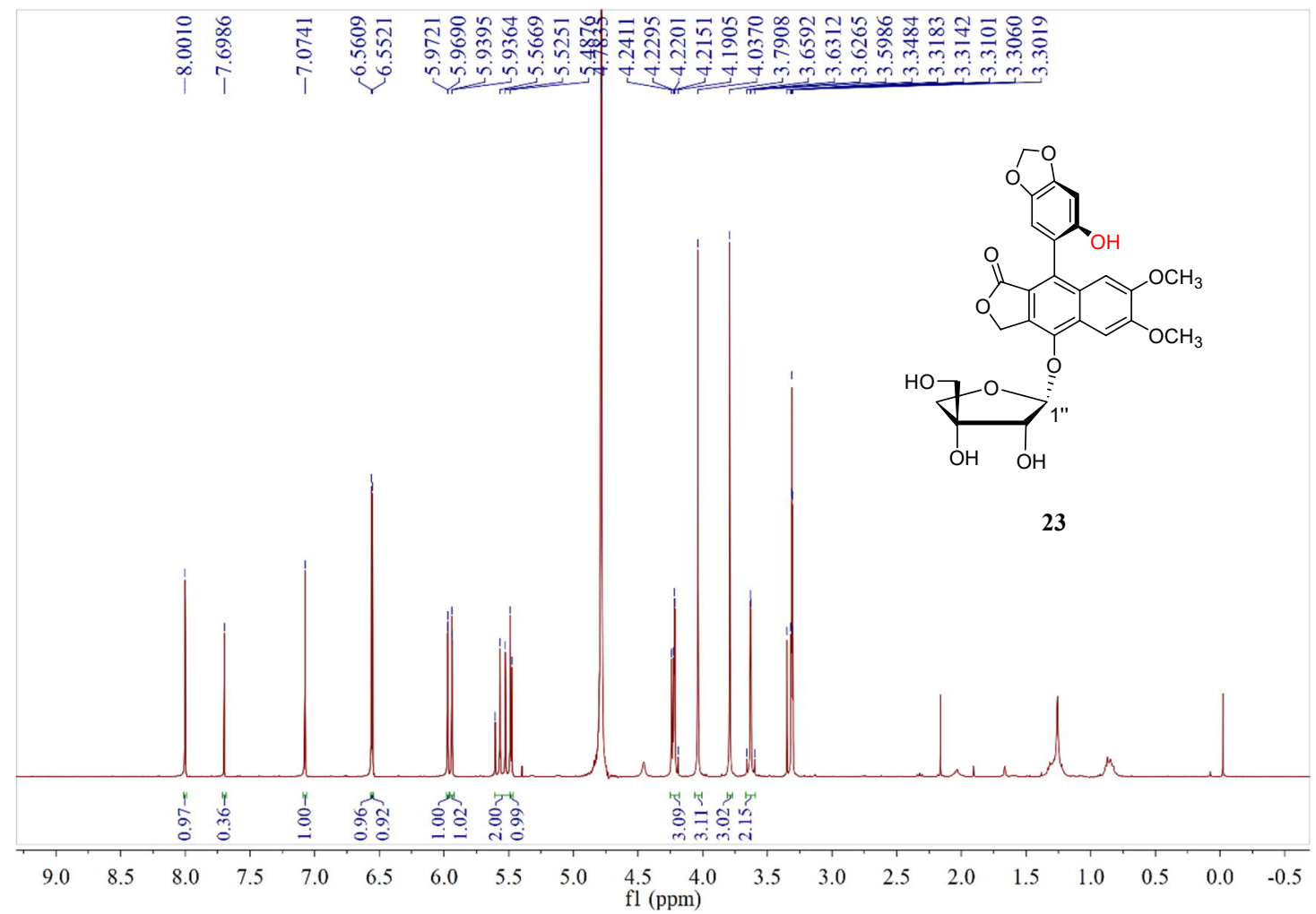

Figure S125. The ${ }^{1} \mathrm{H}$ NMR Spectrum of synthetic 23 in $\mathrm{CD}_{3} \mathrm{OD}(400 \mathrm{MHz})$. 


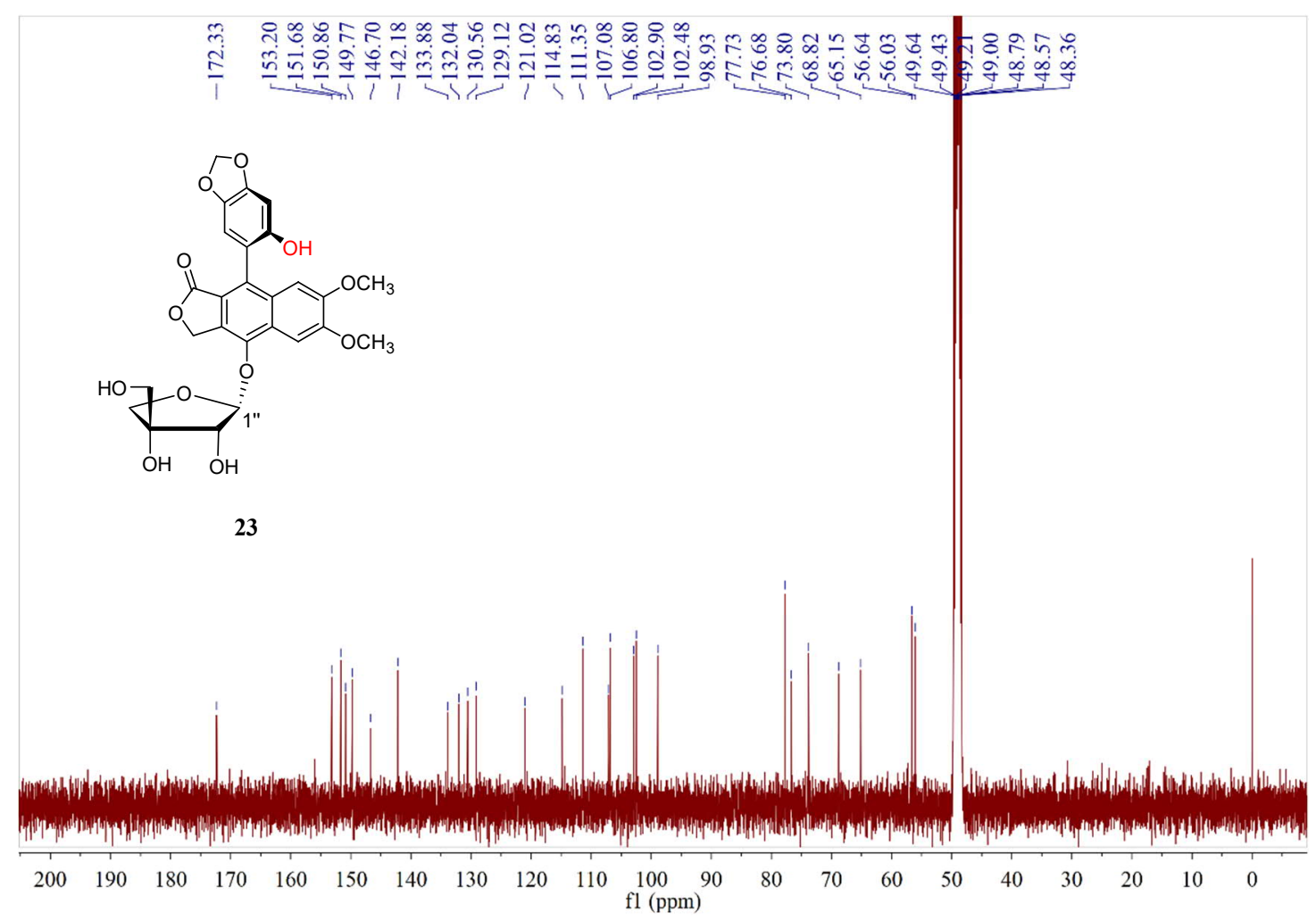

Figure S126. The ${ }^{13} \mathrm{C}\left\{{ }^{1} \mathrm{H}\right\}$ NMR Spectrum of synthetic 23 in $\mathrm{CD}_{3} \mathrm{OD}(100 \mathrm{MHz})$.

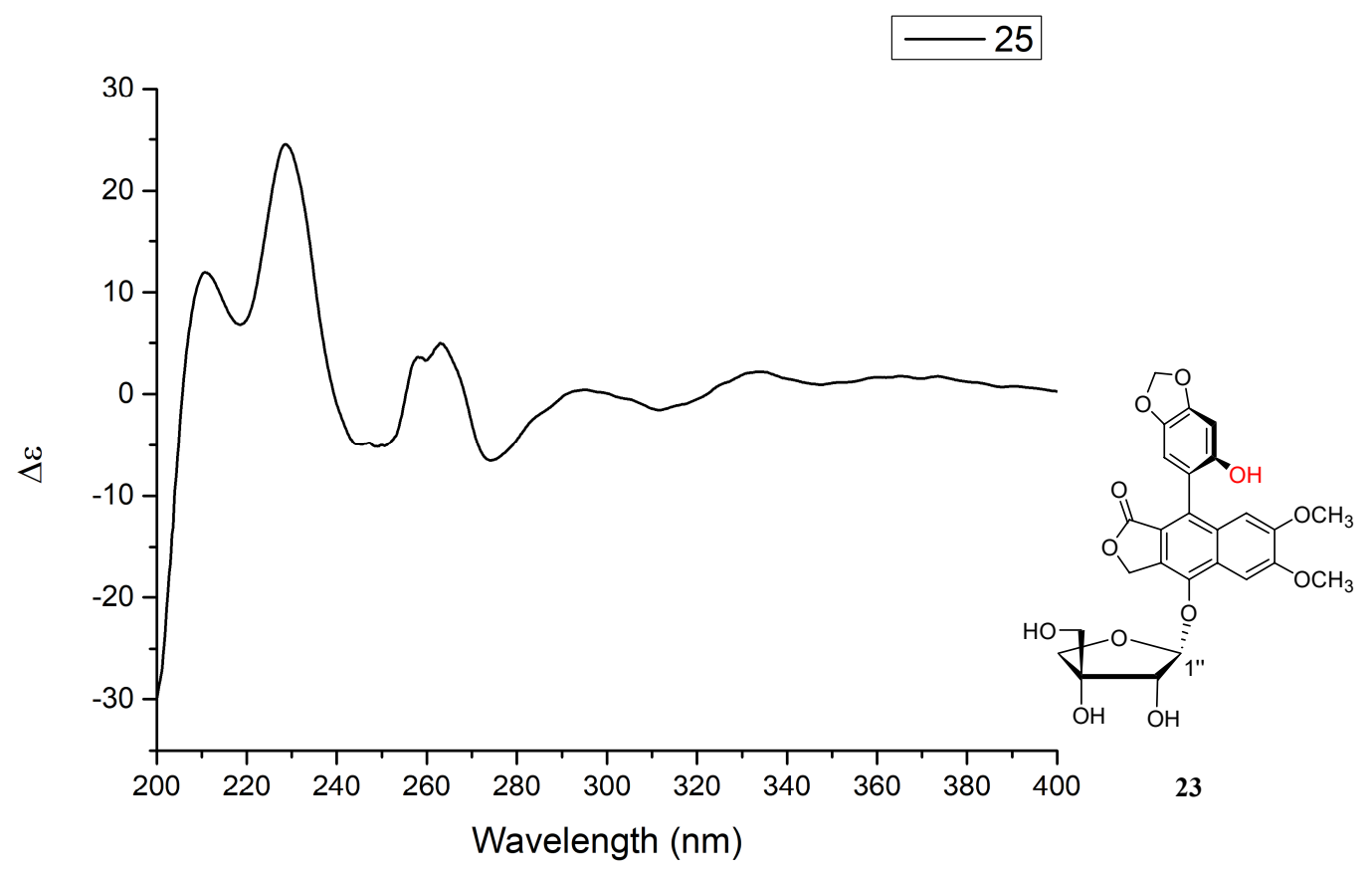

Figure S127. The CD Spectrum of synthetic 23 in $\mathrm{MeOH}$. 


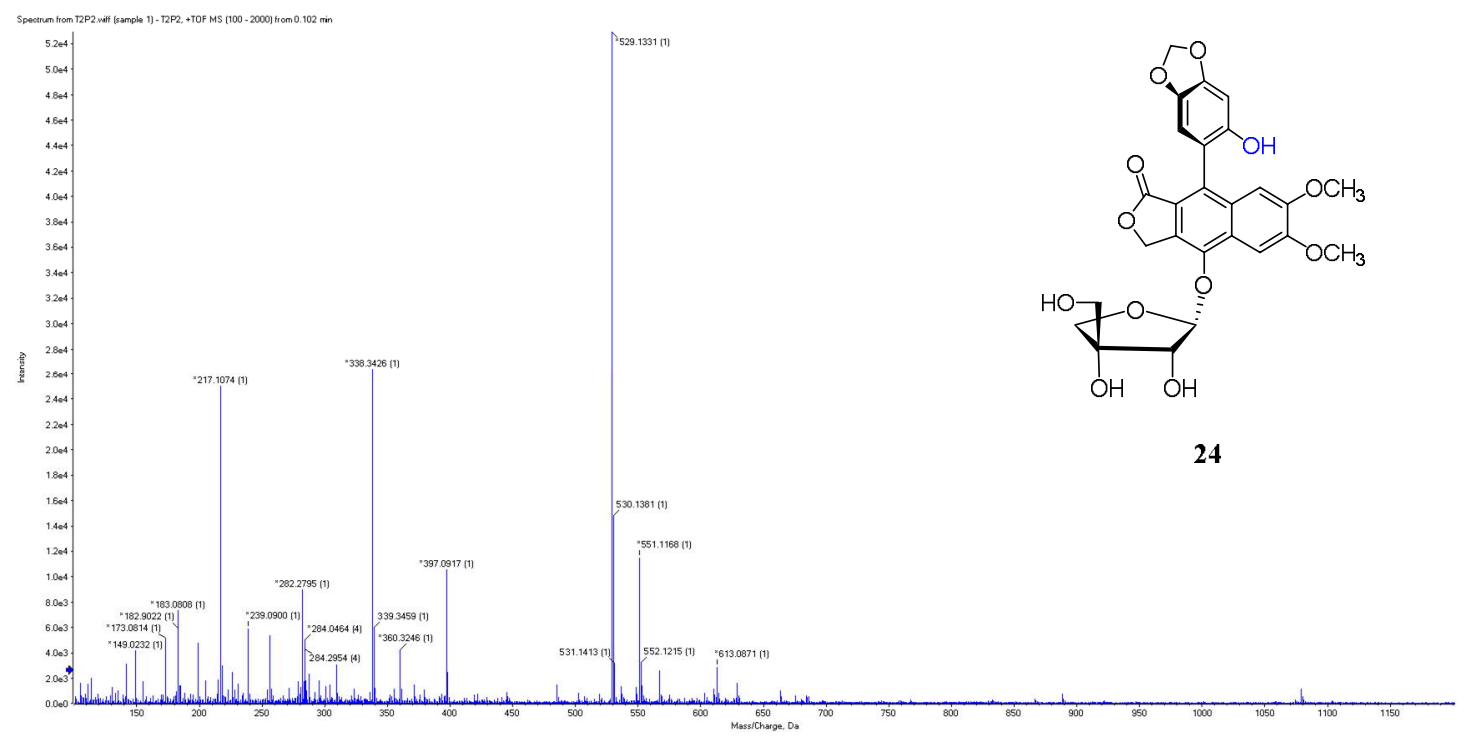

Figure S128. HRESIMS Data of synthetic 24.

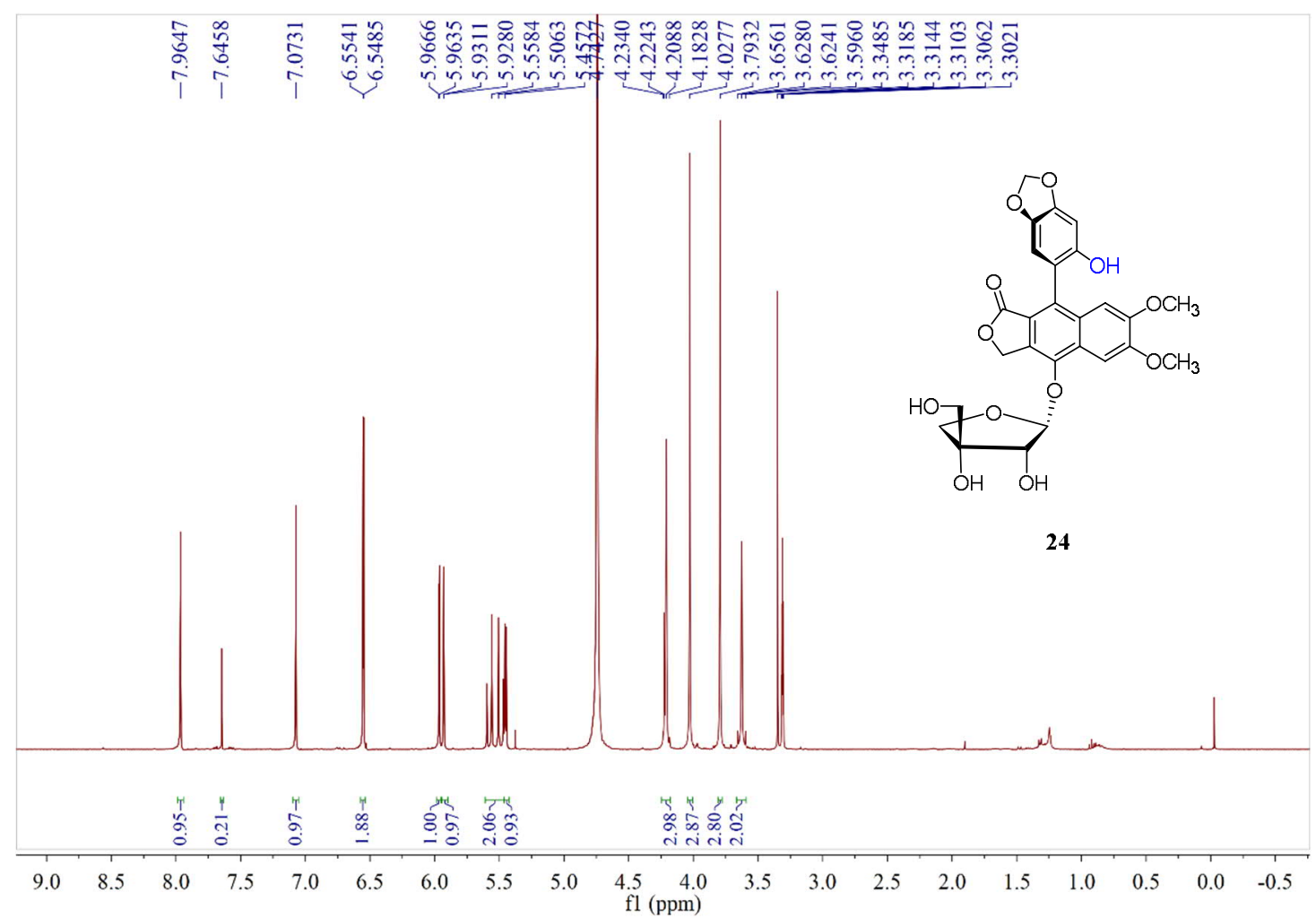

Figure S129. The ${ }^{1} \mathrm{H}$ NMR Spectrum of synthetic 24 in $\mathrm{CD}_{3} \mathrm{OD}(400 \mathrm{MHz})$. 


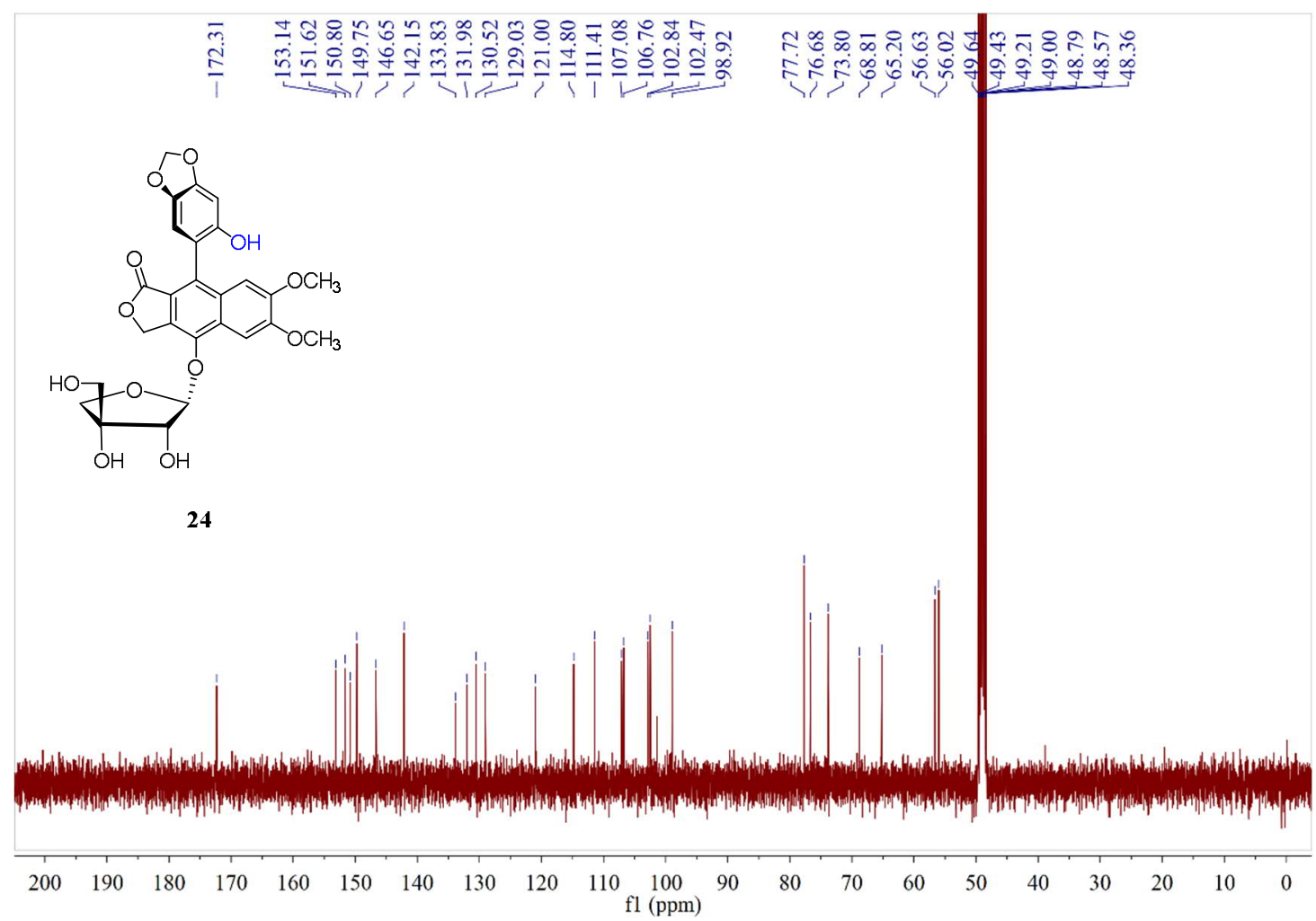

Figure S130. The ${ }^{13} \mathrm{C}\left\{{ }^{1} \mathrm{H}\right\}$ NMR Spectrum of synthetic 24 in $\mathrm{CD}_{3} \mathrm{OD}(100 \mathrm{MHz})$.

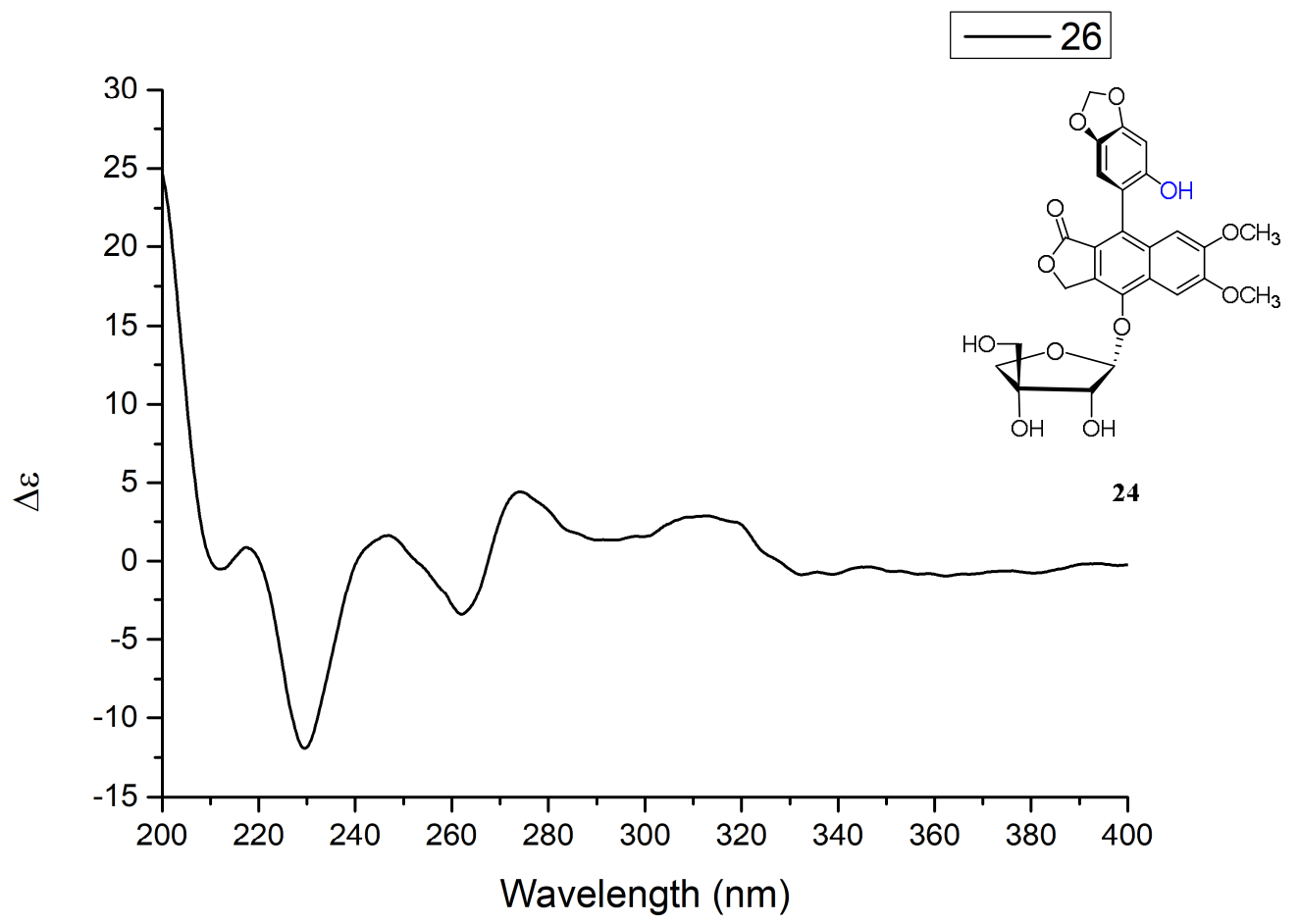

Figure S131. The CD Spectrum of synthetic 24 in $\mathrm{MeOH}$. 